\title{
Construction of Bicyclic 1,2,3-Triazine $N$-Oxides from Aminocyanides
}

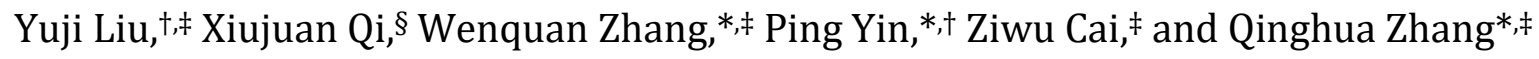

${ }^{\dagger}$ School of Material Science \& Engineering, Beijing Institute of Technology, Beijing 100081, China

${ }^{\ddagger}$ Institute of Chemical Materials, China Academy of Engineering Physics, Mianyang 621900, China

${ }^{\S}$ School of Material Science and Engineering, Southwest University of Science and Technology, Mianyang, 621900, China

\section{Table of Contents}

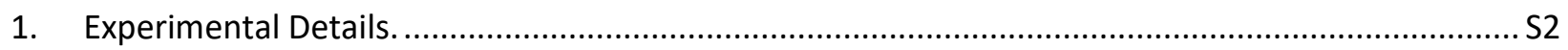

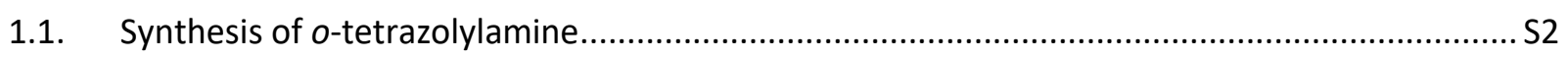

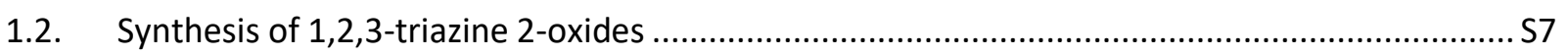

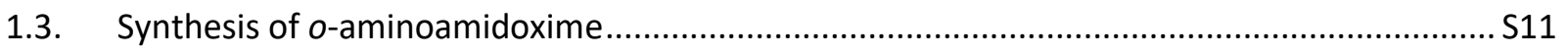

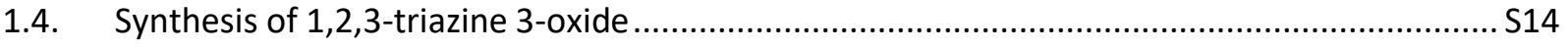

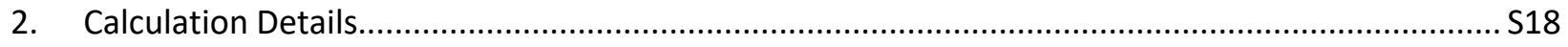

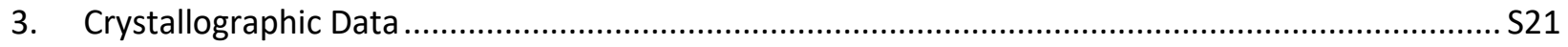

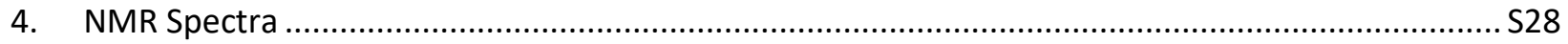

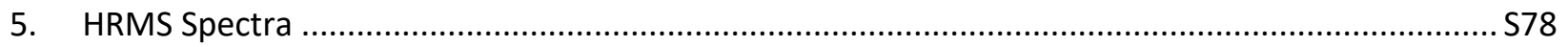

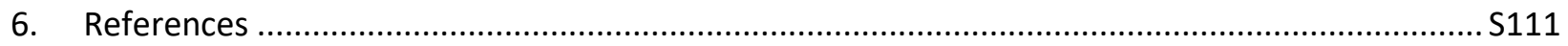




\section{Experimental Details}

Safety precautions:

Some compounds prepared in this study are potentially explosive and may detonate under certain circumstances. Although we encountered no difficulties in handling these materials, it is still advisable to take protective methods (reaction shields, face shields, ear plugs, etc.) for personnel and property safety.

General methods:

All chemical reagents were commercially acquired from Alfa Aesar, Sigma-Aldrich or $\mathrm{TCl}$ and were used without further purification. ${ }^{1} \mathrm{H},{ }^{13} \mathrm{C},{ }^{15} \mathrm{~N},{ }^{1} \mathrm{H}-{ }^{13} \mathrm{C} \mathrm{HSQC},{ }^{1} \mathrm{H}-{ }^{15} \mathrm{~N}$ HSQC NMR spectra were recorded on Bruker Avance 400 instrument. The working frequencies for ${ }^{1} \mathrm{H},{ }^{13} \mathrm{C},{ }^{15} \mathrm{~N}$ were $400.13,100.62,40.55 \mathrm{MHz}$ respectively. ${ }^{13} \mathrm{C}$ and ${ }^{15} \mathrm{~N}$ NMR spectra were proton decoupled using broadband decoupling and inverse gated decoupling, respectively. DMSO- $d_{6}$, Acetone- $d_{6}$ or $\mathrm{D}_{2} \mathrm{SO}_{4}$ were used as solvents and locking solvents. Chemical shifts were given relative to tetramethylsilane (TMS) as internal standard for ${ }^{1} \mathrm{H}$ and ${ }^{13} \mathrm{C}$ and liquid ammonia for ${ }^{15} \mathrm{~N}$ as external standard. Infrared (IR) spectra were recorded on Perkin-Elmer Two IR Spectrometer using $\mathrm{KBr}$ pellet. High resolution mass spectrometry (HRMS) spectra were recorded on Shimadzu LCMS-IT-TOF mass spectrometer or Bruker TOF Premier with electrospray ionization (ESI). Melting points were measured on Büchi Melting Point M-560 at a heat rate of $10{ }^{\circ} \mathrm{C} / \mathrm{min}$. The melting points were not corrected. Elemental Analysis (EA) were performed on Vario MICRO cube elemental analyzer in CHNS mode.

\subsection{Synthesis of $o$-tetrazolylamine}

Synthesis of 5,6-dihydrotetrazolo[1,5-c]quinazoline (2a-1):

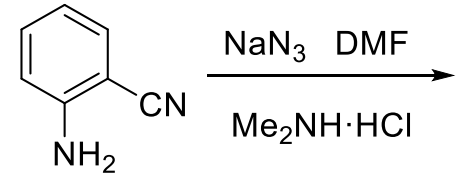

$1 \mathrm{a}$

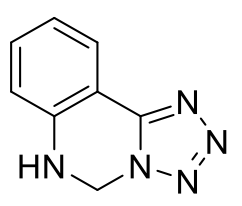

2a-1

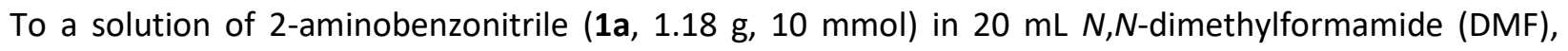
sodium azide $(1.95 \mathrm{~g}, 30 \mathrm{mmol})$ and dimethylamine hydrochloride $(2.56 \mathrm{~g}, 30 \mathrm{mmol})$ were added in one portion. The suspension was heated to $120^{\circ} \mathrm{C}$ with an oil bath, stirred for $12 \mathrm{~h}$, and cooled to rt. The solvent was removed under reduced pressure. The residue was dissolved in $10 \mathrm{~mL}$ water, neutralized with $10 \% \mathrm{HCl}$ and filtered off. The precipitate was washed with water and recrystallized from ethanol to give the product.

White powder. Yield $0.78 \mathrm{~g}(45 \%) . \mathrm{mp} 190-192{ }^{\circ} \mathrm{C} .{ }^{1} \mathrm{H}$ NMR (400 MHz, DMSO-d 6 ): $\delta 7.79(\mathrm{~d}, J=7.6 \mathrm{~Hz}, 1 \mathrm{H})$, $7.38\left(\mathrm{dd}, J_{1}=8.4 \mathrm{~Hz}, J_{2}=7.6 \mathrm{~Hz}, 1 \mathrm{H}\right), 7.18\left(\mathrm{br}, 1 \mathrm{H}\right.$, exchangeable with $\left.\mathrm{D}_{2} \mathrm{O}\right), 6.96(\mathrm{~d}, J=8.4 \mathrm{~Hz}, 1 \mathrm{H}), 6.91$ (dd, $\left.J_{1}=7.6 \mathrm{~Hz}, J_{2}=7.2 \mathrm{~Hz}, 1 \mathrm{H}\right), 5.82$ (br, 2H). ${ }^{13} \mathrm{C} \mathrm{NMR}\left(101 \mathrm{MHz}, \mathrm{DMSO}-\mathrm{d}_{6}\right): \delta 149.2,144.3,133.0,125.0$, 119.1, 115.6, 107.5, 57.8. HRMS (ESI) m/z: [M+ H] $]^{+}$Calcd for $\mathrm{C}_{8} \mathrm{H}_{8} \mathrm{~N}_{5}{ }^{+}$174.0774; Found 174.0782. [M + $\mathrm{Na}]^{+}$Calcd for $\mathrm{C}_{8} \mathrm{H}_{7} \mathrm{~N}_{5} \mathrm{Na}^{+}$196.0594; Found 196.0596. [M $\left.+\mathrm{K}\right]^{+}$Calcd for $\mathrm{C}_{8} \mathrm{H}_{7} \mathrm{~N}_{5} \mathrm{~K}^{+}$212.0333. Found 212.0330. Anal. Calcd for $\mathrm{C}_{8} \mathrm{H}_{7} \mathrm{~N}_{5}$ : C, 55.48; H, 4.07; N, 40.44. Found: $\mathrm{C}, 55.40 ; \mathrm{H}, 4.01 ; \mathrm{N}, 39.64$. 
Synthesis of tetrazolo[1,5-c]quinazolin-5-one (2a-2):

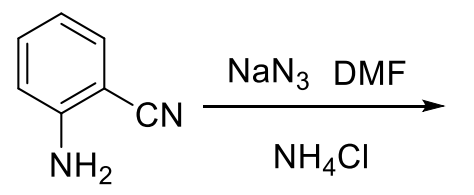

1a<smiles>O=c1[nH]c2ccccc2c2nnnn12</smiles>

2a-2

To a solution of 2-aminobenzonitrile (1a, $1.18 \mathrm{~g}, 10 \mathrm{mmol})$ in $20 \mathrm{~mL} \mathrm{DMF}$, sodium azide (1.30 g, $20 \mathrm{mmol})$ and ammonium chloride $(1.07 \mathrm{~g}, 20 \mathrm{mmol})$ were added in one portion. The suspension was heated to $120^{\circ} \mathrm{C}$ with an oil bath, stirred for $12 \mathrm{~h}$ and cooled to rt. The solvent was removed under reduced pressure. The residue was dissolved in $10 \mathrm{~mL}$ water, neutralized with $10 \% \mathrm{HCl}$ and filtered off. The precipitate was washed with water, and dried in vacuo to give the product.

Yellow powder. Yield $1.63 \mathrm{~g}$ (87\%). $\mathrm{mp} 255-256{ }^{\circ} \mathrm{C}$ (dec.). ${ }^{1} \mathrm{H}$ NMR (400 MHz, DMSO-d 6 ): $\delta 12.69(\mathrm{~s}, 1 \mathrm{H})$, $8.27(\mathrm{~d}, J=7.6 \mathrm{~Hz}, 1 \mathrm{H}), 7.78\left(\mathrm{dd}, J_{1}=8.0 \mathrm{~Hz}, J_{2}=7.6 \mathrm{~Hz}, 1 \mathrm{H}\right), 7.48(\mathrm{~m}, 2 \mathrm{H}) .{ }^{13} \mathrm{C} \mathrm{NMR}\left(101 \mathrm{MHz}, \mathrm{DMSO}-\mathrm{d}_{6}\right)$ : $\delta 150.8,142.2,137.7,134.0,124.8,124.2,116.4,107.8 .{ }^{15} \mathrm{~N} \mathrm{NMR}\left(40 \mathrm{MHz}, \mathrm{DMSO}-\mathrm{d}_{6}\right): \delta 396.7,355.9$, 314.9, 244.1, 128.1. HRMS (ESI) $\mathrm{m} / \mathrm{z}$ : $[\mathrm{M}+\mathrm{H}]^{+}$Calcd for $\mathrm{C}_{8} \mathrm{H}_{6} \mathrm{~N}_{5} \mathrm{O}^{+}$188.0567; Found 188.0574. [M + Na] ${ }^{+}$ Calcd for $\mathrm{C}_{8} \mathrm{H}_{5} \mathrm{~N}_{5} \mathrm{ONa}^{+}$210.0386; Found 210.0373. [M - H] Calcd for $\mathrm{C}_{8} \mathrm{H}_{4} \mathrm{~N}_{5} \mathrm{O}^{-}$186.0421; Found 186.0438. IR: v 3435, 3089, 2983, 2920, 2875, 1752, 1724, 1630, 1551, 1486, 1443, 1390, 1345, 1294, 1259, 1204, $1169,1115,1097,971,903,760,700,661,563,453 \mathrm{~cm}^{-1}$. Anal. Calcd for $\mathrm{C}_{8} \mathrm{H}_{5} \mathrm{~N}_{5} \mathrm{O}: \mathrm{C}, 51.34 ; \mathrm{H}, 2.69 ; \mathrm{N}$, 37.42. Found: $\mathrm{C}, 50.94 ; \mathrm{H}, 2.71 ; \mathrm{N}, 37.04$.

Synthesis of 2-tetrazolylaniline (2a):

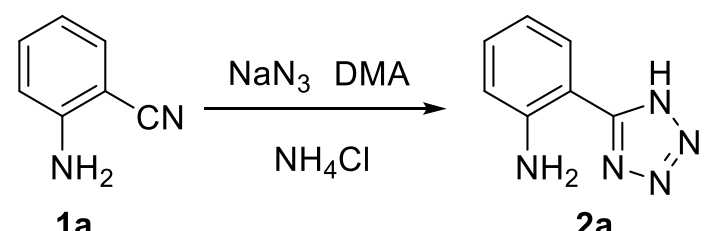

To a solution of 2-aminobenzonitrile (1a, $1.18 \mathrm{~g}, 10 \mathrm{mmol}$ ) in $20 \mathrm{~mL} \mathrm{~N}, \mathrm{~N}$-dimethylacetamide (DMA), sodium azide $(1.30 \mathrm{~g}, 20 \mathrm{mmol})$ and ammonium chloride $(1.07 \mathrm{~g}, 20 \mathrm{mmol})$ were added in one portion. The suspension was heated to $120^{\circ} \mathrm{C}$ with an oil bath, stirred for $12 \mathrm{~h}$ and cooled to rt. The solvent was removed under reduced pressure. The residue was dissolved in $10 \mathrm{~mL}$ water, neutralized with $10 \% \mathrm{HCl}$ and filtered off. The precipitate was washed with water, and dried in vacuo to give the product.

Yellow powder. Yield $1.03 \mathrm{~g}(64 \%) \cdot \mathrm{mp} 169-170{ }^{\circ} \mathrm{C}$ (dec.). ${ }^{1} \mathrm{H}$ NMR $\left(400 \mathrm{MHz}, \mathrm{DMSO}-\mathrm{d}_{6}\right): \delta 7.83$ (d, $J=7.6$ $\mathrm{Hz}, 1 \mathrm{H}), 7.30(\mathrm{t}, J=7.6 \mathrm{~Hz}, 1 \mathrm{H}), 7.00(\mathrm{~d}, J=7.6 \mathrm{~Hz}, 1 \mathrm{H}), 6.79(\mathrm{t}, \mathrm{J}=7.6 \mathrm{~Hz}, 1 \mathrm{H}) .{ }^{13} \mathrm{C}$ NMR $(101 \mathrm{MHz}$, DMSO$\left.\mathrm{d}_{6}\right): \delta 154.8,145.8,131.9,128.4,117.3,116.9,106.0$. HRMS (ESI) m/z: [M - H] Calcd for $\mathrm{C}_{8} \mathrm{H}_{4} \mathrm{~N}_{5} \mathrm{O}^{-}$160.0629; Found 160.0634. IR: v 3435, 3089, 2983, 2920, 2875, 1752, 1724, 1630, 1551, 1486, 1443, 1390, 1345, $1294,1259,1204,1169,1115,1097,971,903,760,700,661,563,453 \mathrm{~cm}^{-1}$. Anal. Calcd for $\mathrm{C}_{7} \mathrm{H}_{7} \mathrm{~N}_{5}: \mathrm{C}_{\text {, }}$ 52.17; H, 4.38; N, 43.45. Found: C, 52.14; $H, 4.24 ; N, 43.26$. 
General procedures for synthesis of $\boldsymbol{o}$-tetrazolylamine $\mathbf{2 b}$-2l:

Compounds $\mathbf{1 b}$ - $\mathbf{1 l}$ (10 mmol) was dissolved in $20 \mathrm{~mL} \mathrm{DMF}$. Sodium azide $(1.30 \mathrm{~g}, 20 \mathrm{mmol})$ and ammonium chloride $(1.07 \mathrm{~g}, 20 \mathrm{mmol})$ was added in one portion. The suspension was heated with an oil bath to $120^{\circ} \mathrm{C}$, stirred for $12 \mathrm{~h}$, and cooled to rt. The solvent was removed under reduced pressure. The residue was dissolved in water, neutralized with $10 \% \mathrm{HCl}$ to $\mathrm{pH}=2$. The precipitate was collected by filtration, washed with water and dried in vacuo to give the product.

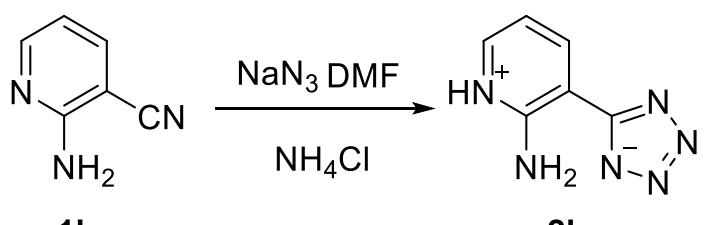

$1 \mathrm{~b}$

2b

3-Tetrazolylpyridin-2-amine (2b). White solid. Yield $1.34 \mathrm{~g}$ (83\%). mp 293-294 ${ }^{\circ} \mathrm{C}$ (dec.). ${ }^{1} \mathrm{H} \mathrm{NMR}(400 \mathrm{MHz}$, DMSO- $\left.\mathrm{d}_{6}\right): \delta 8.30(\mathrm{~d}, J=7.2 \mathrm{~Hz}, 1 \mathrm{H}), 8.10(\mathrm{~d}, J=4.2 \mathrm{~Hz}, 1 \mathrm{H}), 7.70(\mathrm{br}, 2 \mathrm{H}), 6.84\left(\mathrm{dd}, J_{1}=7.2 \mathrm{~Hz}, J_{2}=4.2 \mathrm{~Hz}\right.$, 1H). ${ }^{13} \mathrm{C}$ NMR (101 MHz, DMSO-d d $: \delta 154.9,154.7,146.7,137.4,112.2,104.8$. HRMS (ESI) m/z: [M + H] ${ }^{+}$ Calcd for $\mathrm{C}_{6} \mathrm{H}_{7} \mathrm{~N}_{6}{ }^{+}$163.0727; Found 163.0739. IR: $v$ 3400, 2086, 1673, 1629, 1484, 1455, 1388, 1239, 1066, $798,766,673 \mathrm{~cm}^{-1}$. Anal. Calcd for $\mathrm{C}_{6} \mathrm{H}_{6} \mathrm{~N}_{6}: \mathrm{C}, 44.44 ; \mathrm{H}, 3.73 ; \mathrm{N}, 51.83$. Found: $\mathrm{C}, 44.04 ; \mathrm{H}, 3.69 ; \mathrm{N}, 50.88$.<smiles></smiles>

4-Tetrazolylpyridin-3-amine (2c). White solid. Yield $1.26 \mathrm{~g}$ (78\%). mp 292-293 ${ }^{\circ} \mathrm{C}$ (dec.). ${ }^{1} \mathrm{H}$ NMR (400 MHz, DMSO-d $\left.\mathrm{d}_{6}\right): \delta 8.27(\mathrm{~s}, 1 \mathrm{H}), 8.06(\mathrm{~d}, J=5.6 \mathrm{~Hz}, 1 \mathrm{H}), 7.97(\mathrm{~d}, J=5.6 \mathrm{~Hz}, 1 \mathrm{H}), 5.59$ (br). ${ }^{13} \mathrm{C}$ NMR $(101 \mathrm{MHz}$, DMSO-d $\mathrm{d}_{6}$ ): $\delta 156.0,142.9,133.1,131.3,121.7,119.0$. HRMS (ESI) $\mathrm{m} / \mathrm{z}:[\mathrm{M}+\mathrm{H}]^{+}$Calcd for $\mathrm{C}_{6} \mathrm{H}_{7} \mathrm{~N}_{6}{ }^{+} 163.0727$; Found 163.0723. IR: v 3436, 3330, 1626, 1549, 1507, 1479, 1426, 1330, 1239, 1222, 1150, 1053, 1018, 834, 801, 700, $524 \mathrm{~cm}^{-1}$. Anal. Calcd for $\mathrm{C}_{6} \mathrm{H}_{6} \mathrm{~N}_{6}: \mathrm{C}, 44.44 ; \mathrm{H}, 3.73 ; \mathrm{N}, 51.83$. Found: $\mathrm{C}, 44.12 ; \mathrm{H}, 3.60 ; \mathrm{N}, 51.07$.<smiles>Nc1ccncc1N=Nc1ccncc1-c1nnn[nH]1</smiles>

3-Tetrazolylpyridin-4-amine (2d). White solid. Yield $1.30 \mathrm{~g}(80 \%) . \mathrm{mp} 357-358{ }^{\circ} \mathrm{C}$ (dec.). ${ }^{1} \mathrm{H} \mathrm{NMR}(400 \mathrm{MHz}$, DMSO-d $\left.\mathrm{d}_{6}\right): \delta 13.27(\mathrm{br}, 1 \mathrm{H}), 9.49(\mathrm{br}, 1 \mathrm{H}), 8.90(\mathrm{~s}, 1 \mathrm{H}), 8.71(\mathrm{br}, 1 \mathrm{H}), 8.11(\mathrm{~d}, J=6.8 \mathrm{~Hz}, 1 \mathrm{H}), 7.05(\mathrm{~d}, J=6.8$ $\mathrm{Hz}, 1 \mathrm{H}) .{ }^{13} \mathrm{C}$ NMR $\left(101 \mathrm{MHz}\right.$, DMSO-d $\left.\mathrm{d}_{6}\right): \delta 156.1,155.9,137.6,136.9,111.1,109.7$. HRMS (ESI) m/z: [M + $\mathrm{H}]^{+}$Calcd for $\mathrm{C}_{6} \mathrm{H}_{7} \mathrm{~N}_{6}{ }^{+}$163.0727; Found 163.0740. IR: $v$ 3436, 3330, 1626, 1549, 1479, 1426, 1331, 1239, $1222,1153,1054,1019,834,801,524 \mathrm{~cm}^{-1}$. Anal. Calcd for $\mathrm{C}_{6} \mathrm{H}_{6} \mathrm{~N}_{6}: \mathrm{C}, 44.44 ; \mathrm{H}, 3.73 ; \mathrm{N}, 51.83$. Found: $\mathrm{C}$, 44.23; H, 3.51; N, 50.86 . 


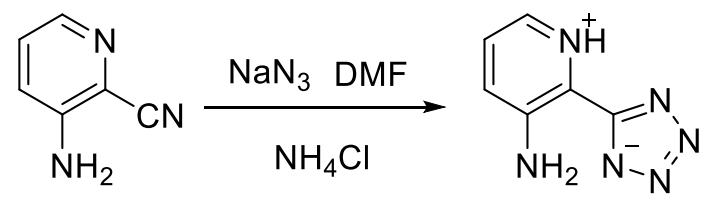

$1 e$

2e

2-Tetrazolylpyridin-3-amine (2e). White solid. Yield $1.31 \mathrm{~g}$ (81\%). mp 293-294 ${ }^{\circ} \mathrm{C}$ (dec.). ${ }^{1} \mathrm{H} \mathrm{NMR}(400 \mathrm{MHz}$, DMSO- $\left.\mathrm{d}_{6}\right): \delta 7.99$ (dd, $\left.J_{1}=4.0 \mathrm{~Hz}, J_{2}=5.2 \mathrm{~Hz}, 1 \mathrm{H}\right), 7.33(\mathrm{~m}, 2 \mathrm{H}) .{ }^{13} \mathrm{C}$ NMR $\left(101 \mathrm{MHz}, \mathrm{DMSO}-\mathrm{d}_{6}\right): \delta 155.1$, 144.1, 137.2, 126.6, 124.3, 124.0. HRMS (ESI) m/z: [M + H] ${ }^{+}$Calcd for $\mathrm{C}_{6} \mathrm{H}_{7} \mathrm{~N}_{6}{ }^{+}$163.0727; Found 163.0740. Anal. Calcd for $\mathrm{C}_{6} \mathrm{H}_{6} \mathrm{~N}_{6}: \mathrm{C}, 44.44 ; \mathrm{H}, 3.73 ; \mathrm{N}, 51.83$. Found: $\mathrm{C}, 44.24 ; \mathrm{H}, 3.44 ; \mathrm{N}, 50.98$.

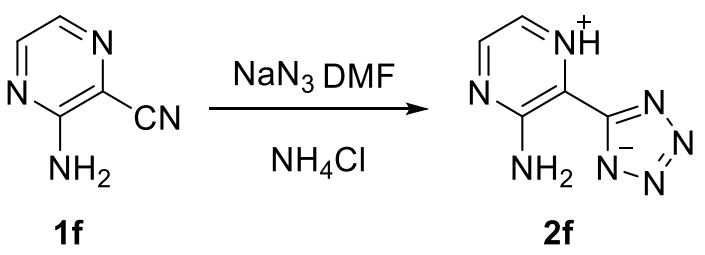

3-Tetrazolylpyrazin-2-amine (2f). Grey solid. Yield $1.19 \mathrm{~g}$ (73\%). mp 360-361 ${ }^{\circ} \mathrm{C}$ (dec.). ${ }^{1} \mathrm{H} \mathrm{NMR} \mathrm{(400} \mathrm{MHz,}$ DMSO-d $\mathrm{d}_{6}$ ): $\delta 8.28$ (d, $\left.J=2.2 \mathrm{~Hz}, 1 \mathrm{H}\right), 7.91(\mathrm{~d}, J=2.2 \mathrm{~Hz}, 1 \mathrm{H}), 7.32(\mathrm{~s}, 2 \mathrm{H}) .{ }^{13} \mathrm{C}$ NMR $\left(101 \mathrm{MHz}, \mathrm{DMSO}-\mathrm{d}_{6}\right): \delta$ 153.7, 153.2, 145.8, 132.3, 121.6. HRMS (ESI) m/z: [M - H] $]^{-}$Calcd for $\mathrm{C}_{5} \mathrm{H}_{4} \mathrm{~N}_{7}{ }^{-}$162.0534; Found 162.0534. IR: $v$ 3436, 3330, 1626, 1550, 1508, 1480, 1426, 1331, 1239, 1222, 1153, 1054, 1019, 835, 801, 748, 700, $524 \mathrm{~cm}^{-1}$. Anal. Calcd for $\mathrm{C}_{5} \mathrm{H}_{5} \mathrm{~N}_{7}: \mathrm{C}, 36.81 ; \mathrm{H}, 3.09 ; \mathrm{N}, 60.10$. Found: $\mathrm{C}, 36.76 ; \mathrm{H}, 3.01 ; \mathrm{N}, 59.98$.

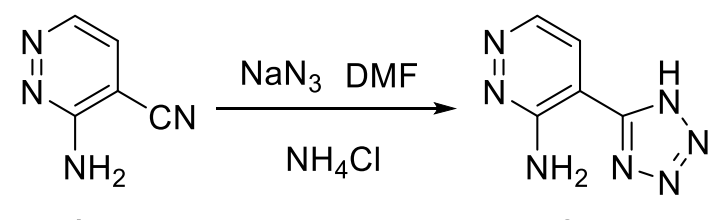

$1 \mathrm{~g}$

$2 \mathrm{~g}$

4-Tetrazolylpyridazin-3-amine (2g). Brick-red solid. Yield $1.21 \mathrm{~g}$ (74\%). mp 273-274 ${ }^{\circ} \mathrm{C}$ (dec.). ${ }^{1} \mathrm{H}$ NMR (400 $\mathrm{MHz}, \mathrm{DMSO}-\mathrm{d}_{6}$ ): $\delta 8.27(\mathrm{~s}, 1 \mathrm{H}), 8.06(\mathrm{~d}, J=5.6 \mathrm{~Hz}, 1 \mathrm{H}), 7.97(\mathrm{~d}, J=5.6 \mathrm{~Hz}, 1 \mathrm{H}), 5.53$ (br). ${ }^{13} \mathrm{C}$ NMR $(101$ $\mathrm{MHz}, \mathrm{DMSO}-\mathrm{d}_{6}$ ): $\delta 155.1,154.0,141.7,126.7,121.1$. HRMS (ESI) $\mathrm{m} / \mathrm{z}:[\mathrm{M}+\mathrm{H}]^{+}$Calcd for $\mathrm{C}_{5} \mathrm{H}_{6} \mathrm{~N}_{7}{ }^{+} 164.0679$; Found 164.0698. IR: $v$ 3468, 3260, 3109, 2675, 1928, 1660, 1606, 1562, 1474, 1453, 1392, 1320, 1287, $1213,1179,1160,1068,1014,985,875,856,781,739,678,601,534 \mathrm{~cm}^{-1}$. Anal. Calcd for $\mathrm{C}_{5} \mathrm{H}_{5} \mathrm{~N}_{7}$ : C, 36.81; H, 3.09; N, 60.10. Found: C, 36.87; H, 2.98; N, 59.99.

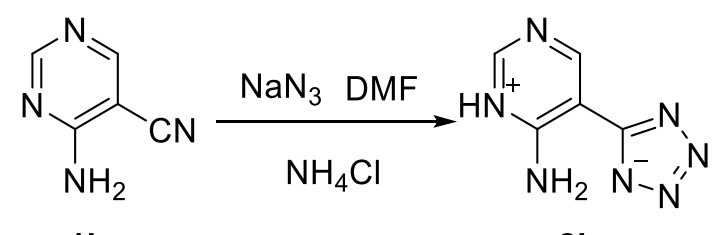

$1 \mathrm{~h}$

$2 \mathrm{~h}$

5-Tetrazolylpyrimidin-4-amine (2h). Yellow solid. Yield $1.26 \mathrm{~g}$ (77\%). mp 329-330 ${ }^{\circ} \mathrm{C}$ (dec.). ${ }^{1} \mathrm{H}$ NMR (400 $\left.\mathrm{MHz}, \mathrm{DMSO}-\mathrm{d}_{6}\right): \delta 8.80(\mathrm{~s}, 1 \mathrm{H}), 8.61(\mathrm{br}, 2 \mathrm{H}), 8.56(\mathrm{~s}, 1 \mathrm{H}) .{ }^{13} \mathrm{C}$ NMR $\left(101 \mathrm{MHz}, \mathrm{DMSO}-\mathrm{d}_{6}\right): \delta$ 160.2, 155.6, 153.3, 148.4, 103.6. HRMS (ESI) $\mathrm{m} / \mathrm{z}$ : $[\mathrm{M}+\mathrm{H}]^{+}$Calcd for $\mathrm{C}_{5} \mathrm{H}_{6} \mathrm{~N}_{7}^{+}$164.0679; Found 164.0694. IR: $v$ 3354, 3182, 3079, 1670, 1631, 1590, 1554, 1516, 1391, 1289, 1133, 1111, 1032, 995, 932, 887, 790, 699, 617, $581,554,515 \mathrm{~cm}^{-1}$. Anal. Calcd for $\mathrm{C}_{5} \mathrm{H}_{5} \mathrm{~N}_{7}: \mathrm{C}, 36.81 ; \mathrm{H}, 3.09 ; \mathrm{N}, 60.10$. Found: $\mathrm{C}, 36.80 ; \mathrm{H}, 2.94 ; \mathrm{N}, 59.98$. 


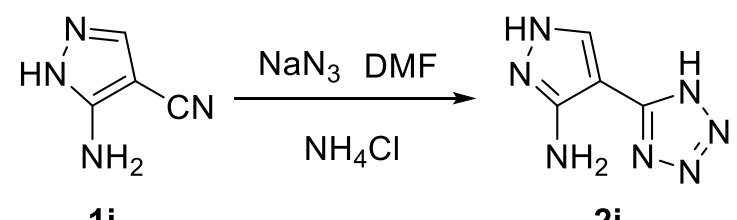

$1 \mathrm{i}$

2i

4-Tetrazolyl-1H-pyrazol-3-amine (2i). ${ }^{1}$ Yellow solid. Yield $1.22 \mathrm{~g}$ (81\%). mp 264-265 ${ }^{\circ} \mathrm{C} .{ }^{1} \mathrm{H}$ NMR (400 MHz, DMSO-d 6 ): $\delta 13.70(\mathrm{br}, 1 \mathrm{H}), 9.94(\mathrm{~s}, 1 \mathrm{H}), 8.39(\mathrm{~s}, 2 \mathrm{H})$. HRMS (ESI) m/z: [M - H] ${ }^{+}$Calcd for $\mathrm{C}_{4} \mathrm{H}_{4} \mathrm{~N}_{7}{ }^{-}$150.0534; Found 150.0526. IR: v 3427, 3339, 3207, 2979, 1623, 1532, 1510, 1385, 1368, 1264, 1071, 1051, 1002, 933, $748,595 \mathrm{~cm}^{-1}$. Anal. Calcd for. $\mathrm{C}_{4} \mathrm{H}_{5} \mathrm{~N}_{7}: \mathrm{C}, 31.79 ; \mathrm{H}, 3.33 ; \mathrm{N}, 64.88$. Found: $\mathrm{C}, 31.74 ; \mathrm{H}, 3.34 ; \mathrm{N}, 64.82$.

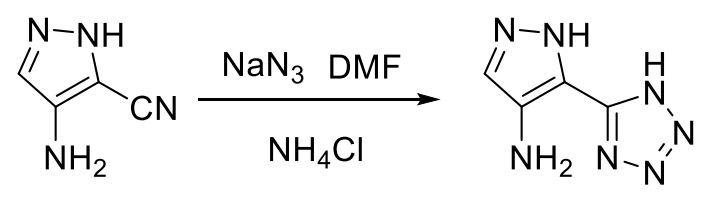

$1 \mathrm{j}$

2j

3-Tetrazolyl-1H-pyrazol-4-amine (2j). ${ }^{2}$ Grey solid. Yield $1.21 \mathrm{~g}$ (80\%). mp 270-271 ${ }^{\circ} \mathrm{C}$ (sub.). HRMS (ESI) $\mathrm{m} / \mathrm{z}:[\mathrm{M}-\mathrm{H}]^{+}$Calcd for $\mathrm{C}_{4} \mathrm{H}_{4} \mathrm{~N}_{7}{ }^{-}$150.0534; Found 150.0536. IR: v 3307, 3252, 2944, 2646, 1636, 1623, 1585, 1551, 1476, 1420, 1377, 1332, 1226, 1212, 1187, 1108, 1068, 994, 936, 977, 754, 648, $620 \mathrm{~cm}^{-1}$. Anal. Calcd for $\mathrm{C}_{4} \mathrm{H}_{5} \mathrm{~N}_{7}$ : C, 31.79; $\mathrm{H}, 3.33 ; \mathrm{N}, 64.88$. Found: $\mathrm{C}, 31.80 ; \mathrm{H}, 3.30 ; \mathrm{N}, 64.90$.

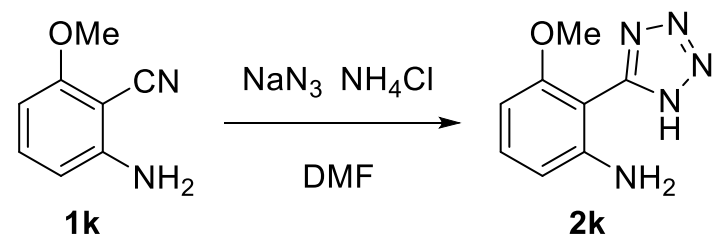

3-Methoxy-2-tetrazolylaniline (2k). Grey solid. Yield $1.57 \mathrm{~g}$ (82\%). mp 122-124 ${ }^{\circ} \mathrm{C} .{ }^{1} \mathrm{H}$ NMR (400 MHz, DMSO-d $\left.\mathrm{d}_{6}\right): 7.39$ (d, $\left.J=8.0 \mathrm{~Hz}, 1 \mathrm{H}\right), 6.98(\mathrm{~d}, J=8.0 \mathrm{~Hz}, 1 \mathrm{H}), 6.72(\mathrm{t}, J=8.0 \mathrm{~Hz}, 1 \mathrm{H}), 3.87(\mathrm{~s}) .{ }^{13} \mathrm{C}$ NMR $(101$ MHz, DMSO-d $\mathrm{d}_{6}$ ): 154.7, 147.0, 137.4, 119.4, 115.4, 111.7, 104.2, 55.6. HRMS (ESI) m/z: [M + H] Calcd for $\mathrm{C}_{8} \mathrm{H}_{10} \mathrm{~N}_{5} \mathrm{O}^{+}$192.0880; Found 192.0885. [M + Na] ${ }^{+}$Calcd for $\mathrm{C}_{8} \mathrm{H}_{9} \mathrm{~N}_{5} \mathrm{ONa}^{+}$214.0699; Found 214.0682. IR: $v$ 3402 , 3285, 3119, 3013, 2974, 2908, 2841, 2778, 2628, 1614, 1602, 1558, 1493, 1473, 1464, 1452, 1441, $1409,1360,1284,1230,1182,1168,1144,1097,1079,1056,1015,906,849,828,795,742,718,701,645$, 583,526 .<smiles>N#Cc1ccc([N+](=O)[O-])cc1N</smiles>

5-Nitro-2-tetrazolylaniline (2I). Orange solid. Yield $1.69 \mathrm{~g}$ (82\%). mp $230-232{ }^{\circ} \mathrm{C}$ (sub.). ${ }^{1} \mathrm{H}$ NMR $(400 \mathrm{MHz}$, DMSO- $\mathrm{d}_{6}$ ): $7.99(\mathrm{~d}, J=8.8 \mathrm{~Hz}, 1 \mathrm{H}), 7.79(\mathrm{~s}, 1 \mathrm{H}), 7.48(\mathrm{~d}, J=8.8 \mathrm{~Hz}, 1 \mathrm{H}), 7.28(\mathrm{br}) .{ }^{13} \mathrm{C} \mathrm{NMR}(101 \mathrm{MHz}$, DMSO$\left.\mathrm{d}_{6}\right): 154.6,149.4,148.0,129.7,110.4,110.3,109.3$. Anal. Calcd for $\mathrm{C}_{7} \mathrm{H}_{6} \mathrm{~N}_{6} \mathrm{O}_{2}: \mathrm{C}, 40.78 ; \mathrm{H}, 2.93 ; \mathrm{N}, 40.76$. Found: $\mathrm{C}, 40.88 ; \mathrm{H}, 3.02 ; \mathrm{N}, 40.48$. 


\subsection{Synthesis of 1,2,3-triazine 2-oxides}

General procedures for synthesis of 1,2,3-triazine 2-oxides 3a-3e, $\mathbf{3 i - 3 \mathbf { I }}$

Nitric acid $\left(d=1.50 \mathrm{~g} / \mathrm{cm}^{3}, 2 \mathrm{~mL}\right.$ ) was added dropwise to trifluoroacetic anhydride $(6 \mathrm{~mL})$ at $-15^{\circ} \mathrm{C}$ cooled with a low-temperature reactor. After addition, the solution was stirred for another $30 \mathrm{~min}$, slowly warmed to $\mathrm{rt}$ and stirred for further $30 \mathrm{~min}$. It was cooled to $-15{ }^{\circ} \mathrm{C}$ again and $\mathbf{2 a - 2 e}, \mathbf{2 i - 2 l}$ ( $3 \mathrm{mmol}$ ) was added in small portions while maintaining a temperature below $-10{ }^{\circ} \mathrm{C}$. The solution was stirred at $-15{ }^{\circ} \mathrm{C}$ for $2 \mathrm{~h}$ before poured onto crushed ice. The aqueous solution was extracted with ethyl acetate. The organic phases were combined, neutralized with sodium bicarbonate, dried over anhydrous magnesium sulfate and concentrated under reduced pressure to give 1,2,3-triazine 2-oxides.

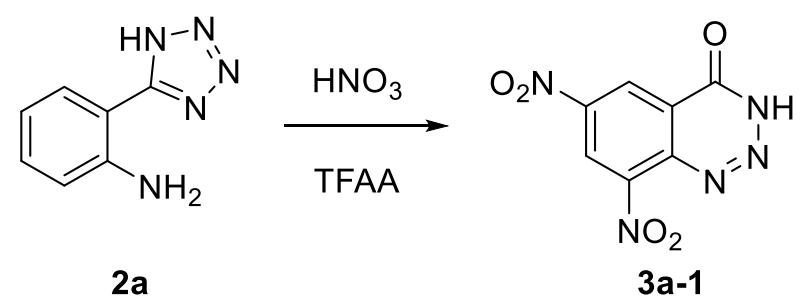

6,8-Dinitrobenzo[1,2,3]triazin-4(3H)-one (3a-1). Yellow solid. Yield $0.43 \mathrm{~g}(60 \%) . \mathrm{mp} 162-163{ }^{\circ} \mathrm{C}$ (dec.). ${ }^{1} \mathrm{H}$ NMR (400 MHz, DMSO-d 6 ): $\delta 9.36(\mathrm{~s}, 1 \mathrm{H}), 8.79(\mathrm{~s}, 1 \mathrm{H}) .{ }^{13} \mathrm{C}$ NMR $\left(101 \mathrm{MHz}, \mathrm{DMSO}-\mathrm{d}_{6}\right): \delta$ 165.4, 144.7, 144.0, 141.0, 126.2, 122.3, 109.2. HRMS (ESI) m/z: [M - H] Calcd for $\mathrm{C}_{7} \mathrm{H}_{2} \mathrm{~N}_{5} \mathrm{O}_{5}{ }^{-}$252.0011; Found 252.0011.

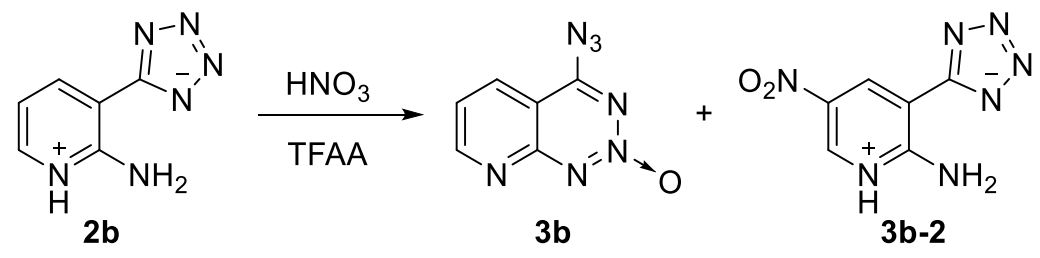

4-Azidopyrido[2,3-d][1,2,3]triazine 2-oxide (3b). Yellow solid. Yield $0.50 \mathrm{~g}(89 \%) . \mathrm{mp} 167-168{ }^{\circ} \mathrm{C}$ (dec.). ${ }^{1} \mathrm{H}$ NMR (400 MHz, DMSO-d 6 ): $\delta 9.22(\mathrm{~d}, J=4.0 \mathrm{~Hz}, 1 \mathrm{H}), 8.41(\mathrm{~d}, J=4.0 \mathrm{~Hz}, 1 \mathrm{H}), 7.72(\mathrm{t}, J=4.0 \mathrm{~Hz}, 1 \mathrm{H}) .{ }^{13} \mathrm{C}$ NMR $(101 \mathrm{MHz}$, DMSO-d $): \delta 164.5,160.5,158.4,132.4,124.6,104.9$. HRMS (ESI) m/z: $[M+H]^{+}$Calcd for $\mathrm{C}_{6} \mathrm{H}_{4} \mathrm{~N}_{7} \mathrm{O}^{+}$190.0472; Found 190.0482. [M + Na] ${ }^{+}$Calcd for $\mathrm{C}_{6} \mathrm{H}_{3} \mathrm{~N}_{7} \mathrm{NaO}^{+}$212.0291; Found 212.0310. IR: $v$ 3439, 2230, 2169, 2131, 1612, 1590, 1538, 1479, 1430, 1402, 1353, 1340, 1273, 1192, 1114, 1034, 1000, $921,872,791,745,726,652,578,541 \mathrm{~cm}^{-1}$. Anal. Calcd for $\mathrm{C}_{6} \mathrm{H}_{3} \mathrm{~N}_{7} \mathrm{O}: \mathrm{C}, 38.10 ; \mathrm{H}, 1.60 ; \mathrm{N}, 51.84$. Found: $\mathrm{C}$, $38.01 ; \mathrm{H}, 1.55 ; \mathrm{N}, 51.49$.

5-Nitro-3-tetrazolyl-pyridin-2-amine (3b-2) was separated by column chromatography from the nitration mixture of $\mathbf{2 b}$. Its potassium salt was obtained during the neutralization process. Yellow solid. Yield 0.03 g (5\%). mp 287-288 ${ }^{\circ} \mathrm{C}$ (dec.). ${ }^{1} \mathrm{H}$ NMR (400 MHz, Acetone-d 6 ): $\delta: 9.51$ (s, 1H), 9.05 (s, 2H), 9.01 (s, $1 \mathrm{H}$ ), 8.91 $(\mathrm{s}, 2 \mathrm{H}) .{ }^{13} \mathrm{C}$ NMR $\left(101 \mathrm{MHz}\right.$, Acetone-d $\left.\mathrm{d}_{6}\right): \delta 160.7,151.4,146.6,136.3,131.5,130.8$. HRMS (ESI) m/z: [M $\mathrm{H}]^{-}$Calcd for $\mathrm{C}_{6} \mathrm{H}_{4} \mathrm{~N}_{7} \mathrm{O}_{2}^{-}$206.0432; Found 206.0451. Anal. Calcd for $\mathrm{C}_{6} \mathrm{H}_{5} \mathrm{~N}_{7} \mathrm{O}_{2}: \mathrm{C}, 34.79 ; \mathrm{H}, 2.43 ; \mathrm{N}, 47.33$. Found: $\mathrm{C}, 34.62 ; \mathrm{H}, 2.58 ; \mathrm{N}, 47.04$. 
Synthesis of 6-nitro-4-oxo-4,8-dihydropyrido[2,3-d][1,2,3]triazine 2-oxide (3b-1):

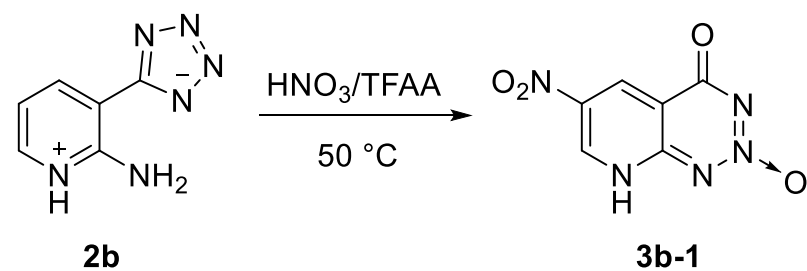

Nitric acid $\left(d=1.50 \mathrm{~g} / \mathrm{cm}^{3}, 2 \mathrm{~mL}\right)$ was added dropwise to trifluoroacetic anhydride $(6 \mathrm{~mL})$ at $-15^{\circ} \mathrm{C}$ cooled with a low-temperature reactor. After the addition, the solution was stirred for another 30 min, slowly warmed to rt and stirred for a further $30 \mathrm{~min}$. It was cooled to $-15^{\circ} \mathrm{C}$ again and 3-tetrazolylpyridin-2-amine (2b, $0.486 \mathrm{~g}, 3 \mathrm{mmol}$ ) was added in small portions to it while maintaining a temperature below $-10^{\circ} \mathrm{C}$. The solution was stirred at $-15^{\circ} \mathrm{C}$ for $30 \mathrm{~min}$, slowly warmed to room temperature, and stirred for another 30 min. The solution was gradually heated with an oil bath to $50{ }^{\circ} \mathrm{C}$ and stirred for $1 \mathrm{~h}$. It was then cooled to room temperature and poured onto crushed ice. The aqueous solution was extracted with ethyl acetate. The organic phases were combined, neutralized with sodium bicarbonate, dried over anhydrous magnesium sulfate and concentrated under reduced pressure to give the product.

Yellow solid. Yield $0.40 \mathrm{~g}(64 \%)$. mp 346-347 ${ }^{\circ} \mathrm{C}$ (dec.). ${ }^{1} \mathrm{H}$ NMR (400 MHz, DMSO-d 6 ): $\delta 9.83$ (d, $J=2.4 \mathrm{~Hz}$, 1H), 8.95 (d, $J=2.4 \mathrm{~Hz}, 1 \mathrm{H}) .{ }^{13} \mathrm{C}$ NMR (101 MHz, DMSO-d 6 ): $\delta 165.6,160.2,154.5,142.1,128.5,104.0$. HRMS (ESI) m/z: [M - H] ${ }^{-}$Calcd for $\mathrm{C}_{6} \mathrm{H}_{2} \mathrm{~N}_{5} \mathrm{O}_{4}{ }^{-}$208.0112; Found 208.0123. Anal. Calcd for $\mathrm{C}_{6} \mathrm{H}_{3} \mathrm{~N}_{5} \mathrm{O}_{4}$ : C, 34.46; $H, 1.45 ; \mathrm{N}, 33.49$. Found: $\mathrm{C}, 34.44 ; \mathrm{H}, 1.49 ; \mathrm{N}, 33.19$.

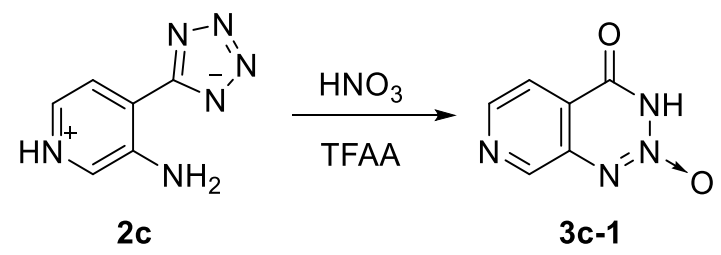

4-Oxo-3,4-dihydropyrido[3,4- $d][1,2,3]$ triazine 2-oxide (3c-1). Yellow solid. Yield $0.41 \mathrm{~g}(83 \%)$. mp 195$196{ }^{\circ} \mathrm{C}$ (dec.). ${ }^{1} \mathrm{H}$ NMR (400 MHz, DMSO-d 6 ): $\delta 11.97$ (s, 1H), 9.15 (s, 1H), 8.65 (s, 1H), $7.86(\mathrm{~s}, 1 \mathrm{H}) .{ }^{13} \mathrm{C}$ NMR (101 MHz, DMSO-d $\mathrm{d}_{6}$ ): $\delta$ 166.9, 155.2, 147.5, 145.1, 131.3, 130.4. HRMS (ESI) m/z: [M - H] Calcd for $\mathrm{C}_{6} \mathrm{H}_{3} \mathrm{~N}_{4} \mathrm{O}_{2}{ }^{-}$163.0261; Found 163.0260. IR: $v$ 3435, 1638, 1530, 1385, 1261, 1162, 1134, 1047, 871, 794, 704, $674,535,467 \mathrm{~cm}^{-1}$. Anal. Calcd for $\mathrm{C}_{6} \mathrm{H}_{4} \mathrm{~N}_{4} \mathrm{O}_{2}: \mathrm{C}, 43.91 ; \mathrm{H}, 2.46 ; \mathrm{N}, 34.14$. Found: $\mathrm{C}, 43.94 ; \mathrm{H}, 2.50 ; \mathrm{N}, 34.10$.

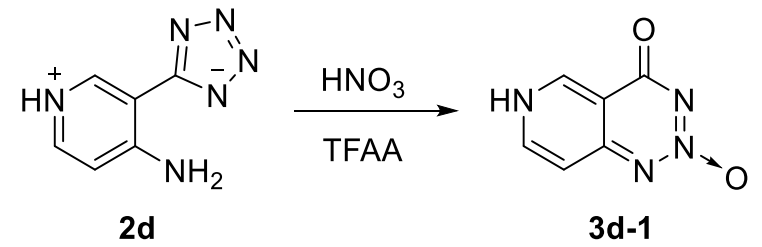

4-Oxo-4,6-dihydropyrido[4,3-d][1,2,3]triazine 2-oxide (3d-1). Yellow solid. Yield $0.41 \mathrm{~g}(84 \%)$. mp 196$197{ }^{\circ} \mathrm{C}$ (dec.). ${ }^{1} \mathrm{H}$ NMR (400 MHz, DMSO-d 6 ): $\delta 9.12(\mathrm{~s}, 1 \mathrm{H}), 8.51(\mathrm{~d}, J=6.6 \mathrm{~Hz}, 1 \mathrm{H}), 7.50(\mathrm{~d}, J=6.6 \mathrm{~Hz}, 1 \mathrm{H})$. ${ }^{13} \mathrm{C}$ NMR $\left(101 \mathrm{MHz}\right.$, DMSO-d 6 ): $\delta 167.9,157.9,142.9,140.9,116.2,109.8$. HRMS (ESI) m/z: $[\mathrm{M}+\mathrm{H}]^{+}$Calcd for $\mathrm{C}_{6} \mathrm{H}_{5} \mathrm{~N}_{4} \mathrm{O}_{2}{ }^{+}$165.0407; Found 165.0407. [M + Na] ${ }^{+}$Calcd for $\mathrm{C}_{6} \mathrm{H}_{4} \mathrm{~N}_{4} \mathrm{NaO}_{2}{ }^{+}$187.0226; Found 187.0219. [M 
- H] - Calcd for $\mathrm{C}_{6} \mathrm{H}_{3} \mathrm{~N}_{4} \mathrm{O}_{2}{ }^{-}$163.0261; Found 163.0266. Anal. Calcd for $\mathrm{C}_{6} \mathrm{H}_{4} \mathrm{~N}_{4} \mathrm{O}_{2}: \mathrm{C}, 43.91 ; \mathrm{H}, 2.46 ; \mathrm{N}, 34.14$. Found: $\mathrm{C}, 44.01 ; \mathrm{H}, 2.38 ; \mathrm{N}, 34.04$.

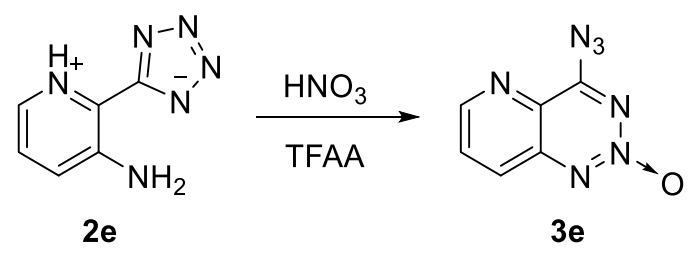

4-Azidopyrido[3,2-d] [1,2,3]triazine 2-oxide (3e). Yellow solid. Yield $0.47 \mathrm{~g}(83 \%) . \mathrm{mp} 187-188{ }^{\circ} \mathrm{C}$ (dec.). ${ }^{1} \mathrm{H}$ NMR $\left(400 \mathrm{MHz}, \mathrm{DMSO}-\mathrm{d}_{6}\right): \delta 9.03(\mathrm{~d}, J=4.2 \mathrm{~Hz}, 1 \mathrm{H}) 8.28(\mathrm{~d}, J=8.0 \mathrm{~Hz}, 1 \mathrm{H}) 8.10\left(\mathrm{dd}, J_{1}=8.0 \mathrm{~Hz}, J_{2}=4.2\right.$ $\mathrm{Hz}, 1 \mathrm{H}) .{ }^{13} \mathrm{C}$ NMR $(101 \mathrm{MHz}$, DMSO-d 6 ): $\delta 164.4,152.4,149.9,146.2,131.8,131.3,125.2$. Anal. Calcd for $\mathrm{C}_{6} \mathrm{H}_{3} \mathrm{~N}_{7} \mathrm{O}: \mathrm{C}, 38.10 ; \mathrm{H}, 1.60 ; \mathrm{N}, 51.84$. Found: $\mathrm{C}, 38.01 ; \mathrm{H}, 1.69 ; \mathrm{N}, 51.78$.

General procedures for synthesis of nitramines $\mathbf{3 f - 1} \cdot \mathrm{H}_{2} \mathrm{O}-\mathbf{3 k - 1} \cdot \mathrm{H}_{2} \mathrm{O}$ :

Nitric acid $\left(d=1.50 \mathrm{~g} / \mathrm{cm}^{3}, 5 \mathrm{~mL}\right)$ was added dropwise to sulfuric acid $(98 \%, 5 \mathrm{~mL})$ at $-5{ }^{\circ} \mathrm{C}$ cooled with an ice-salt bath. $\boldsymbol{O}$-Tetrazolylamines $\mathbf{2 f}-\mathbf{2} \mathbf{k}$ ( $3 \mathrm{mmol}$ ) was added to the solution in small portions. The reaction mixture was stirred at $-5{ }^{\circ} \mathrm{C}$ for $2 \mathrm{~h}$ and poured onto crushed ice afterwards. The formed precipitate was collected by filtration, washed with a small amount of ice-water and dried in air to give the nitramines.

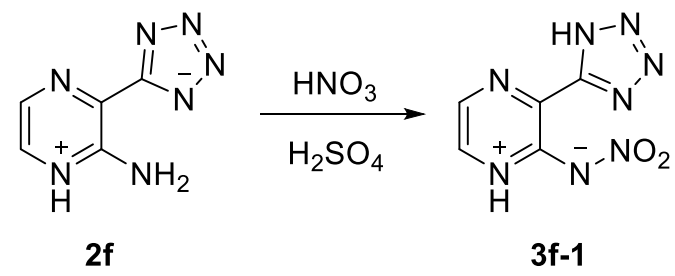

3-Tetrazolylpyrazin-2-nitramide hydrate (3f-1 $\cdot \mathrm{H}_{2} \mathrm{O}$ ). Yellow solid. Yield $0.44 \mathrm{~g}(64 \%)$. mp $136-137^{\circ} \mathrm{C}$ (dec.). ${ }^{1} \mathrm{H}$ NMR $(400 \mathrm{MHz}$, DMSO-d 6 ): $\delta 8.98$ (d, $J=2.4 \mathrm{~Hz}, 1 \mathrm{H}), 8.91$ (d, $\left.J=2.4 \mathrm{~Hz}, 1 \mathrm{H}\right) .{ }^{13} \mathrm{C} \mathrm{NMR}(101 \mathrm{MHz}$, DMSO$\left.\mathrm{d}_{6}\right): \delta$ 153.0, 145.5, 144.4, 142.2, 137.2. HRMS (ESI) m/z: [M - H] Calcd for $\mathrm{C}_{5} \mathrm{H}_{3} \mathrm{~N}_{8} \mathrm{O}_{2}{ }^{-}$207.0384; Found. 207.0388. IR: $v 3438,1633,1573,1432,1385,1208,1137,1123,1057,1008,894,831,782,771,735,632$, $591 \mathrm{~cm}^{-1}$. Anal. Calcd for $\mathrm{C}_{5} \mathrm{H}_{6} \mathrm{~N}_{8} \mathrm{O}_{3}: \mathrm{C}, 26.55 ; \mathrm{H}, 2.67 ; \mathrm{N}, 49.55$. Found: $\mathrm{C}, 26.45 ; \mathrm{H}, 2.59 ; \mathrm{N}, 49.12$.

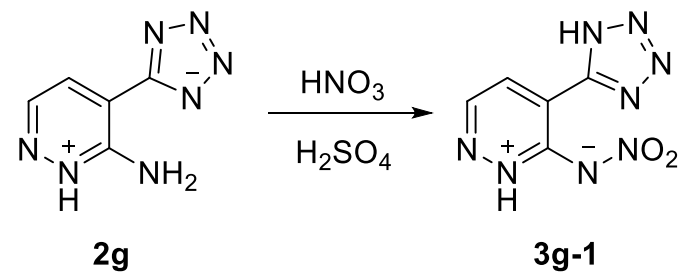

4-Tetrazolylpyridazin-3-nitramide hydrate $\left(\mathbf{3 g}-1 \cdot \mathrm{H}_{2} \mathrm{O}\right)$. Yellow solid. Yield $0.41 \mathrm{~g}(58 \%) . \mathrm{mp} 213.7-213.8{ }^{\circ} \mathrm{C}$ (dec.). ${ }^{1} \mathrm{H}$ NMR (400 MHz, DMSO-d $\left.\mathrm{d}_{6}\right): \delta 8.92(\mathrm{~s}, 1 \mathrm{H}) 8.46(\mathrm{~d}, J=4.0 \mathrm{~Hz}, 1 \mathrm{H}) .{ }^{13} \mathrm{C}$ NMR $\left(101 \mathrm{MHz}, \mathrm{DMSO}-\mathrm{d}_{6}\right)$ : $\delta$ 150.6, 149.8, 145.5, 132.3, 123.6. HRMS (ESI) m/z: [M - H] Calcd for $\mathrm{C}_{5} \mathrm{H}_{3} \mathrm{~N}_{8} \mathrm{O}_{2}^{-}$207.0384; Found. 207.0386. IR: $v$ 3550, 3467, 3205, 3057, 1609, 1575, 1559, 1446, 1348, 1190, 1135, 1094, 1064, 1007, 879, $830,778,766,730,655,586,521 \mathrm{~cm}^{-1}$. Anal. Calcd for $\mathrm{C}_{5} \mathrm{H}_{6} \mathrm{~N}_{8} \mathrm{O}_{3}$ : C, 26.55; H, 2.67; N, 49.55. Found: $\mathrm{C}$, $26.52 ; \mathrm{H}, 2.70 ; \mathrm{N}, 49.54$. 


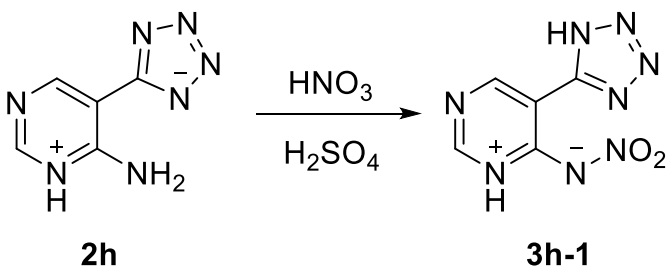

5-Tetrazolylpyrimidin-4-nitramide hydrate $\left(3 \mathrm{~h}-1 \cdot \mathrm{H}_{2} \mathrm{O}\right)$. Yellow solid. Yield $0.42 \mathrm{~g}(62 \%) . \mathrm{mp} 115-116{ }^{\circ} \mathrm{C}$ (dec.). ${ }^{1} \mathrm{H}$ NMR (400 MHz, DMSO-d 6 ): $\delta 8.83(\mathrm{~s}, 1 \mathrm{H}), 8.54(\mathrm{~s}, 1 \mathrm{H}), 8.06(\mathrm{br}) .{ }^{13} \mathrm{C}$ NMR $\left(101 \mathrm{MHz}, \mathrm{DMSO}-\mathrm{d}_{6}\right)$ : $\delta$ 159.1, 153.1, 152.5, 148.7, 112.2. IR: v 3435, 3052, 1725, 1657, 1629, 1563, 1543, 1385, 1261, 1223, $1118,1023,897,841,807,785,703,676,608,578,549,534 \mathrm{~cm}^{-1}$. Anal. Calcd for $\mathrm{C}_{5} \mathrm{H}_{6} \mathrm{~N}_{8} \mathrm{O}_{3}: \mathrm{C}, 26.55 ; \mathrm{H}$, 2.67; N, 49.55. Found: C, 26.54; H, 2.72; N, 49.12 .

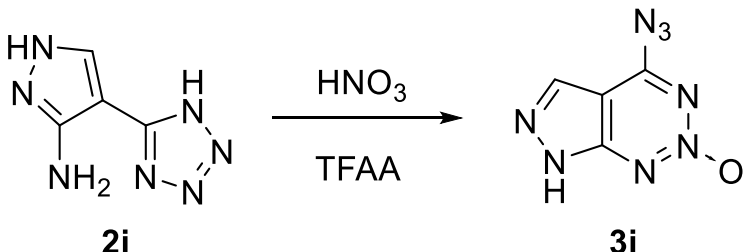

4-azido-7H-pyrazolo[3,4- $d][1,2,3]$ triazine 2-oxide (3i). Yellow solid. Yield $0.42 \mathrm{~g}$ (79\%). $\mathrm{mp} 164-165{ }^{\circ} \mathrm{C}$ (dec.). ${ }^{1} \mathrm{H}$ NMR (400 MHz, DMSO-d $\mathrm{d}_{6}$ ): $\delta 8.43(\mathrm{~s}, 1 \mathrm{H}), 5.41$ (br). ${ }^{13} \mathrm{C}$ NMR $\left(101 \mathrm{MHz}, \mathrm{DMSO}-\mathrm{d}_{6}\right): \delta 157.8,156.3$, 132.1, 96.1. $\mathrm{HRMS}$ (ESI) m/z: [M - H] ' Calcd for $\mathrm{C}_{4} \mathrm{HN}_{8} \mathrm{O}^{-}$177.0279; Found. 177.0297. IR: $v$ 3279, 3115, 2249, 2209, 2163, 1765, 1601, 1558, 1490, 1419, 1385, 1361, 1320, 1288, 1204, 1110, 1046, 993, 941, 885, 849, $779,750,731,706,664,614,570,541 \mathrm{~cm}^{-1}$. Anal. Calcd for $\mathrm{C}_{4} \mathrm{H}_{2} \mathrm{~N}_{8} \mathrm{O}: \mathrm{C}, 26.97 ; \mathrm{H}, 1.13 ; \mathrm{N}, 62.91$. Found: $\mathrm{C}$, $27.04 ; \mathrm{H}, 1.10 ; \mathrm{N}, 63.01$.

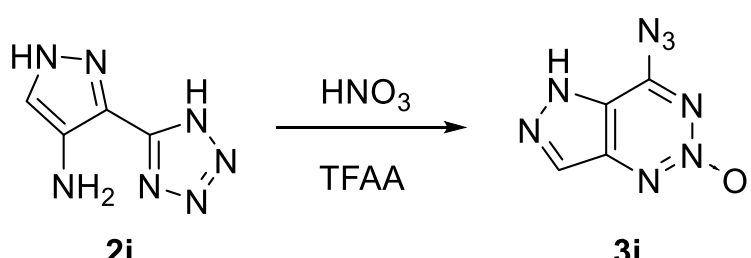

4-Azido-5H-pyrazolo[4,3-d][1,2,3]triazine 2-oxide (3j). ${ }^{2}$ Yellow solid. Yield $0.47 \mathrm{~g}(89 \%)$. Anal. Calcd for $\mathrm{C}_{4} \mathrm{H}_{2} \mathrm{~N}_{8} \mathrm{O}: \mathrm{C}, 26.97 ; \mathrm{H}, 1.13 ; \mathrm{N}, 62.91$. Found: C, 27.04; $\mathrm{H}, 1.15 ; \mathrm{N}, 63.00$.<smiles>Nc1cc([N+](=O)[O-])ccc1-c1nnn[nH]1</smiles>

5,7-Dinitro-4-oxo-3,4-dihydrobenzo[1,2,3]triazine 2-oxide (3l-1). Orange-yellow solid. Yield $0.49 \mathrm{~g}$ (64\%). mp 171-172 ${ }^{\circ} \mathrm{C}$ (dec.). ${ }^{1} \mathrm{H}$ NMR $\left(400 \mathrm{MHz}\right.$, DMSO-d 6 ): $\delta 8.74(\mathrm{~s}, 1 \mathrm{H}), 8.72(\mathrm{~s}, 1 \mathrm{H}) .{ }^{13} \mathrm{C}$ NMR $(101 \mathrm{MHz}$, DMSO$\left.\mathrm{d}_{6}\right): \delta 165.0,151.6,147.2,139.0,122.8,121.0,109.4$. HRMS (ESI) m/z: [M - H] Calcd for $\mathrm{C}_{7} \mathrm{H}_{2} \mathrm{~N}_{5} \mathrm{O}_{6}{ }^{-}$ 252.0011; Found. 252.0013. 


\subsection{Synthesis of $o$-aminoamidoxime}

General procedure for the synthesis of $4 \mathrm{a}-\mathbf{4 m}$ :

To a solution of $1 \mathrm{a}-1 \mathrm{~m}$ (10 mmol) in $10 \mathrm{~mL}$ methanol, aqueous hydroxylamine $(50 \%, 0.61 \mathrm{~mL}, 10 \mathrm{mmol})$ was added to it. The solution was stirred at $\mathrm{rt}$ for $1 \mathrm{~h}$, the precipitate that formed was filtered off, washed with a small amount of water and dried in air to give the $o$-aminoamidoximes.

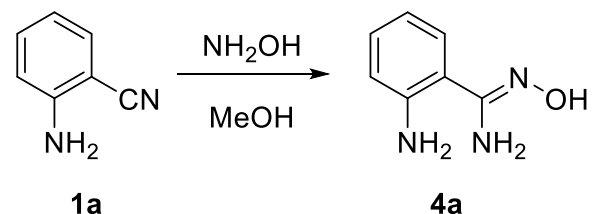

2-Amino- $N$ '-hydroxybenzimidamide (4a). Violet solid. Yield $1.28 \mathrm{~g}$ (85\%). mp 56-57 ${ }^{\circ} \mathrm{C} .{ }^{1} \mathrm{H} \mathrm{NMR}(400 \mathrm{MHz}$, DMSO-d 6 ): $\delta 9.57(\mathrm{~s}, 1 \mathrm{H}), 7.36(\mathrm{~d}, J=7.6 \mathrm{~Hz}, 1 \mathrm{H}), 7.02\left(\mathrm{dd}, J_{1}=8.0 \mathrm{~Hz}, J_{2}=7.2 \mathrm{~Hz}, 1 \mathrm{H}\right), 6.66(\mathrm{~d}, J=8.0 \mathrm{~Hz}$, $1 \mathrm{H}), 6.52(\mathrm{t}, J=7.6 \mathrm{~Hz}, 1 \mathrm{H}), 6.20(\mathrm{br}, 2 \mathrm{H}), 5.72(\mathrm{~s}, 2 \mathrm{H}) .{ }^{13} \mathrm{C} \mathrm{NMR}\left(101 \mathrm{MHz}, \mathrm{DMSO}-\mathrm{d}_{6}\right): \delta 152.9,146.8,129.0$, 127.3, 115.5, 114.9, 114.3. HRMS (ESI) m/z: $[\mathrm{M}+\mathrm{H}]^{+}$Calcd for $\mathrm{C}_{7} \mathrm{H}_{10} \mathrm{~N}_{3} \mathrm{O}^{+}$152.0818; Found 152.0828. IR: $v$ $3338,2807,1636,1537,1378,1247,1151,1078,1003,913,756,722,621,533 \mathrm{~cm}^{-1}$. Anal. Calcd for $\mathrm{C}_{7} \mathrm{H}_{9} \mathrm{~N}_{3} \mathrm{O}: \mathrm{C}, 55.62 ; \mathrm{H}, 6.00 ; \mathrm{N}, 27.80$. Found: $\mathrm{C}, 55.40 ; \mathrm{H}, 5.93 ; \mathrm{N}, 27.08$.

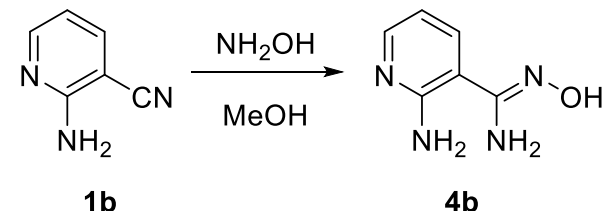

2-Amino- $N$ '-hydroxynicotinimidamide (4b). Yellow solid. Yield $1.11 \mathrm{~g}(73 \%) . \mathrm{mp} 145-147^{\circ} \mathrm{C}$ (dec.). ${ }^{1} \mathrm{H}$ NMR (400 MHz, DMSO-d $\mathrm{d}_{6}$ ): $\delta 9.82(\mathrm{~s}, 1 \mathrm{H}), 7.82(\mathrm{t}, J=2.8 \mathrm{~Hz}, 1 \mathrm{H}), 7.10(\mathrm{~d}, J=2.8 \mathrm{~Hz}, 2 \mathrm{H}), 6.64(\mathrm{~s}, 2 \mathrm{H}), 5.92(\mathrm{~s}$, 2H). ${ }^{13} \mathrm{C}$ NMR (101 MHz, DMSO-d 6 ): $\delta$ 156.6, 151.4, 147.8, 134.8, 111.2, 109.1. HRMS (ESI) m/z: [M + H] ${ }^{+}$ Calcd for $\mathrm{C}_{6} \mathrm{H}_{9} \mathrm{~N}_{4} \mathrm{O}^{+}$153.0771; Found 153.0783. IR: v 3430, 3364, 3267, 2814, 1930, 1651, 1590, 1455, 1399, $1309,1269,1148,1082,1063,967,933,879,798,776,731,654,606,570,534,509 \mathrm{~cm}^{-1}$. Anal. Calcd for $\mathrm{C}_{6} \mathrm{H}_{8} \mathrm{~N}_{4} \mathrm{O}: \mathrm{C}, 47.36 ; \mathrm{H}, 5.30 ; \mathrm{N}, 36.82$, Found: $\mathrm{C}, 47.34 ; \mathrm{H}, 5.49 ; \mathrm{N}, 36.17$.

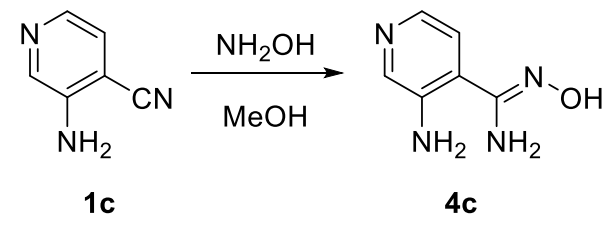

3-Amino- $N$ '-hydroxyisonicotinimidamide (4c). White solid. Yield $1.06 \mathrm{~g}(70 \%) . \mathrm{mp} 193-195{ }^{\circ} \mathrm{C}$ (dec.). ${ }^{1} \mathrm{H}$ $\operatorname{NMR}\left(400 \mathrm{MHz}, \mathrm{DMSO}-\mathrm{d}_{6}\right): \delta 9.98(\mathrm{~s}, 1 \mathrm{H}), 8.06(\mathrm{~s}, 1 \mathrm{H}), 7.74(\mathrm{~d}, J=5.2 \mathrm{~Hz}, 1 \mathrm{H}), 7.33(\mathrm{~d}, J=5.2 \mathrm{~Hz}, 1 \mathrm{H}), 6.45$ $(\mathrm{s}, 2 \mathrm{H}), 5.96(\mathrm{~s}, 2 \mathrm{H}) .{ }^{13} \mathrm{C}$ NMR $\left(101 \mathrm{MHz}, \mathrm{DMSO}-\mathrm{d}_{6}\right): \delta 151.6,142.1,138.6,135.8,120.1,118.6$. HRMS (ESI) $\mathrm{m} / \mathrm{z}:[\mathrm{M}+\mathrm{H}]^{+}$Calcd for $\mathrm{C}_{6} \mathrm{H}_{9} \mathrm{~N}_{4} \mathrm{O}^{+}$153.0771; Found 153.0778. IR: $v$ 3426, 3367, 3312, 3256, 2744, 1653, $1602,1498,1427,1381,1342,1309,1247,1069,973,883,854,809,680,666 \mathrm{~cm}^{-1}$. Anal. Calcd for $\mathrm{C}_{6} \mathrm{H}_{8} \mathrm{~N}_{4} \mathrm{O}: \mathrm{C}, 47.36 ; \mathrm{H}, 5.30 ; \mathrm{N}, 36.82$. Found: $\mathrm{C}, 47.30 ; \mathrm{H}, 5.24 ; \mathrm{N}, 36.76$. 


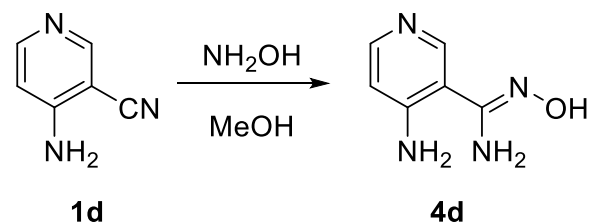

4-Amino- $N$ '-hydroxynicotinimidamide (4d). White solid. Yield $1.77 \mathrm{~g}$ (77\%). mp 230-231 ${ }^{\circ} \mathrm{C}$ (dec.). ${ }^{1} \mathrm{H}$ NMR (400 MHz, DMSO-d d $: \delta 9.78(\mathrm{~s}, 1 \mathrm{H}), 8.39(\mathrm{~s}, 1 \mathrm{H}), 7.93(\mathrm{~d}, J=5.2 \mathrm{~Hz}, 1 \mathrm{H}), 7.04(\mathrm{~s}, 1 \mathrm{H}), 6.57(\mathrm{~d}, J=5.2 \mathrm{~Hz}$, $1 \mathrm{H}), 5.91(\mathrm{~s}, 2 \mathrm{H}) .{ }^{13} \mathrm{C}$ NMR $\left(101 \mathrm{MHz}, \mathrm{DMSO}-\mathrm{d}_{6}\right): \delta 151.8,151.5,148.4,147.9,110.3,109.6$. HRMS (ESI) $\mathrm{m} / \mathrm{z}:[\mathrm{M}+\mathrm{H}]^{+}$Calcd for $\mathrm{C}_{6} \mathrm{H}_{9} \mathrm{~N}_{4} \mathrm{O}^{+}$153.0771; Found 153.0776. [M - H] Calcd for $\mathrm{C}_{6} \mathrm{H}_{7} \mathrm{~N}_{4} \mathrm{O}^{-}$151.0625; Found 151.0659. Anal. Calcd for $\mathrm{C}_{6} \mathrm{H}_{8} \mathrm{~N}_{4} \mathrm{O}: \mathrm{C}, 47.36 ; \mathrm{H}, 5.30 ; \mathrm{N}, 36.82$. Found: $\mathrm{C}, 47.24 ; \mathrm{H}, 5.40 ; \mathrm{N}, 36.70$.

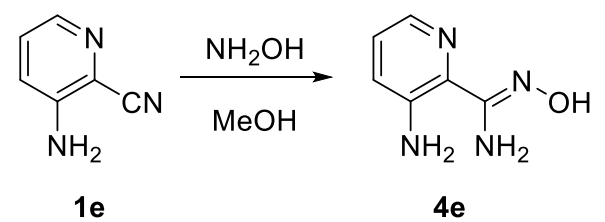

3-Amino- $N$ '-hydroxypicolinimidamide (4e). White solid. Yield $1.08 \mathrm{~g}$ (71\%). mp 233-235 ${ }^{\circ} \mathrm{C}$ (dec.). ${ }^{1} \mathrm{H}$ NMR $\left(400 \mathrm{MHz}, \mathrm{DMSO}-\mathrm{d}_{6}\right): \delta 9.82(\mathrm{~s}, 1 \mathrm{H}), 7.82(\mathrm{t}, J=2.8 \mathrm{~Hz}, 1 \mathrm{H}), 7.10(\mathrm{~d}, J=2.8 \mathrm{~Hz}, 2 \mathrm{H}), 6.64(\mathrm{~s}, 2 \mathrm{H}), 5.92(\mathrm{~s}$, 2H). ${ }^{13} \mathrm{C}$ NMR (101 MHz, DMSO-d 6 ): $\delta$ 153.4, 143.4, 135.3, 129.7, 124.1, 122.5. HRMS (ESI) m/z: [M + H] ${ }^{+}$ Calcd for $\mathrm{C}_{6} \mathrm{H}_{9} \mathrm{~N}_{4} \mathrm{O}^{+}$153.0771; Found 153.0765. [M + Na] ${ }^{+}$Calcd for $\mathrm{C}_{6} \mathrm{H}_{8} \mathrm{~N}_{4} \mathrm{NaO}^{+}$175.0590; Found 175.0592. IR: $v$ 3364, 3328, 3267, 2855, 2813, 1650, 1589, 1455, 1393, 1309, 1268, 1237, 1147, 1083, 1063, 933, 879, $798,776,729,653,602,570,533,509 \mathrm{~cm}^{-1}$. Anal. Calcd for $\mathrm{C}_{6} \mathrm{H}_{8} \mathrm{~N}_{4} \mathrm{O}: \mathrm{C}, 47.36 ; \mathrm{H}, 5.30 ; \mathrm{N}, 36.82$. Found: $\mathrm{C}$, 47.19; $\mathrm{H}, 5.54 ; \mathrm{N}, 36.11$.

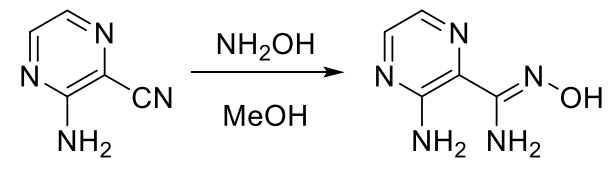

$1 f$

$4 \mathbf{f}$

3-Amino- $N$ '-hydroxypyrazine-2-carboximidamide (4f). White solid. Yield $0.99 \mathrm{~g}(65 \%) . \mathrm{mp} 181-182{ }^{\circ} \mathrm{C} .{ }^{1} \mathrm{H}$ NMR (400 MHz, DMSO-d $\left.)_{6}\right): \delta 10.18(\mathrm{~s}, 1 \mathrm{H}), 8.01(\mathrm{~d}, J=2.4 \mathrm{~Hz}, 1 \mathrm{H}), 7.82(\mathrm{~d}, J=2.4 \mathrm{~Hz}, 1 \mathrm{H}), 7.44(\mathrm{br}, 2 \mathrm{H})$, $5.97(\mathrm{~s}, 2 \mathrm{H}) .{ }^{13} \mathrm{C}$ NMR $\left(101 \mathrm{MHz}, \mathrm{DMSO}-\mathrm{d}_{6}\right): \delta 153.1,151.6,142.4,130.4,127.1$. HRMS (ESI) $\mathrm{m} / \mathrm{z}:[\mathrm{M}+\mathrm{H}]^{+}$ Calcd for $\mathrm{C}_{5} \mathrm{H}_{8} \mathrm{~N}_{5} \mathrm{O}^{+}$154.0723; Found 154.0740. IR: $v$ 3509, 3459, 3417, 3382, 3344, 3304, 3156, 2802, 1641, $1583,1482,1456,1443,1393,1323,1234,1158,1097,1052,963,953,914,852,835,809,709,665,562$, $519,478 \mathrm{~cm}^{-1}$. Anal. Calcd for $\mathrm{C}_{5} \mathrm{H}_{7} \mathrm{~N}_{5} \mathrm{O}: \mathrm{C}, 39.21 ; \mathrm{H}, 4.61 ; \mathrm{N}, 45.73$. Found: $\mathrm{C}, 39.08 ; \mathrm{H}, 4.59 ; \mathrm{N}, 44.93$.

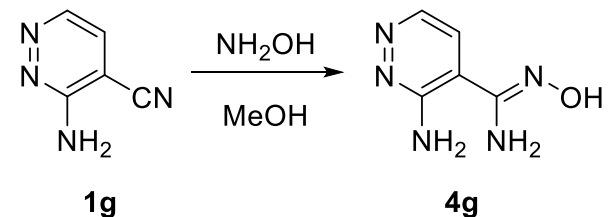

3-Amino- $N$ '-hydroxypyridazine-4-carboximidamide (4g). White solid. Yield $1.02 \mathrm{~g}(67 \%) . \mathrm{mp} 239-240{ }^{\circ} \mathrm{C}$ (dec.). ${ }^{1} \mathrm{H}$ NMR (400 MHz, DMSO-d 6 ): $\delta 10.36(\mathrm{~s}, 1 \mathrm{H}), 8.52$ (d, $\left.J=4.8 \mathrm{~Hz}, 1 \mathrm{H}\right), 7.57(\mathrm{~d}, J=4.8 \mathrm{~Hz}, 1 \mathrm{H}), 7.31$ $(\mathrm{s}, 2 \mathrm{H}), 6.14(\mathrm{~s}, 2 \mathrm{H}) .{ }^{13} \mathrm{C}$ NMR (101 MHz, DMSO-d 6 ): $\delta 157.3,150.0,142.1,123.2,113.4$. HRMS (ESI) $\mathrm{m} / \mathrm{z}$ : $[\mathrm{M}+\mathrm{H}]^{+}$Calcd for $\mathrm{C}_{5} \mathrm{H}_{8} \mathrm{~N}_{5} \mathrm{O}^{+}$154.0723; Found 154.0730. [M + Na] ${ }^{+}$Calcd for $\mathrm{C}_{5} \mathrm{H}_{7} \mathrm{~N}_{5} \mathrm{NaO}^{+}$176.0543; Found 
176.0553. $[\mathrm{M}+\mathrm{K}]^{+}$Calcd for $\mathrm{C}_{5} \mathrm{H}_{7} \mathrm{KN}_{5} \mathrm{O}^{+}$192.0282; Found 192.0287. [M - H] Calcd for $\mathrm{C}_{5} \mathrm{H}_{6} \mathrm{~N}_{5} \mathrm{O}^{-}$152.0578; Found 152.0589. IR: $v$ 3425, 3294, 3188, 3058, 2750, 1642, 1586, 1497, 1458, 1389, 1305, 1252, 1174, $1054,981,864,800,780,680,608,509 \mathrm{~cm}^{-1}$. Anal. Calcd for $\mathrm{C}_{5} \mathrm{H}_{7} \mathrm{~N}_{5} \mathrm{O}: \mathrm{C}, 39.21 ; \mathrm{H}, 4.61 ; \mathrm{N}, 45.73$. Found: C, 39.11; H, 4.69; N, 44.90 .<smiles>COO</smiles>

$1 \mathrm{~h}$<smiles>N/C(=N\O)c1cncnc1N</smiles>

$4 \mathrm{~h}-\mathrm{Z}$<smiles>N/C(=N/O)c1cncnc1N</smiles>

4h-E

4-Amino- $N^{\prime}$-hydroxypyrimidine-5-carboximidamide (4h-Z/E). Yellow solid. Yield $1.04 \mathrm{~g}(68 \%) .{ }^{1} \mathrm{H}$ NMR (400 MHz, DMSO-d $\left.\mathrm{d}_{6}\right): \delta 10.04(\mathrm{~s}, 1 \mathrm{H}), 8.67(\mathrm{~s}, 0.7 \mathrm{H}), 8.61(\mathrm{~s}, 0.8 \mathrm{H}), 8.56(\mathrm{~s}, 1 \mathrm{H}), 8.39(\mathrm{~s}, 1 \mathrm{H}), 7.66(\mathrm{br}, 2 \mathrm{H}), 6.10$ (br, 2H), 7.65 (br, 1.6H), $6.10(\mathrm{~s}, 2 \mathrm{H}) .{ }^{13} \mathrm{C}$ NMR (101 MHz, DMSO-d 6 ): $\delta 162.3,161.0,160.6,159.8,157.2$, 152.6, 149.8, 115.2, 107.2, 89.5. HRMS (ESI) m/z: [M + H] Calcd for $\mathrm{C}_{5} \mathrm{H}_{8} \mathrm{~N}_{5} \mathrm{O}^{+}$154.0723; Found 154.0740. [M - H] Calcd for $\mathrm{C}_{5} \mathrm{H}_{6} \mathrm{~N}_{5} \mathrm{O}^{-}$152.0578; Found 152.0592. IR: $v$ 3483, 3447, 3382, 3326, 2794, 1642, 1582, $1500,1456,1384,1375,1296,1257,1161,1102,1069,1036,927,875,850,804,752,652,556 \mathrm{~cm}^{-1}$. Anal. Calcd for $\mathrm{C}_{5} \mathrm{H}_{7} \mathrm{~N}_{5} \mathrm{O}$ : C, 39.21; $\mathrm{H}, 4.61 ; \mathrm{N}, 45.73$. Found: $\mathrm{C}, 39.05 ; \mathrm{H}, 4.53 ; \mathrm{N}, 45.05$.

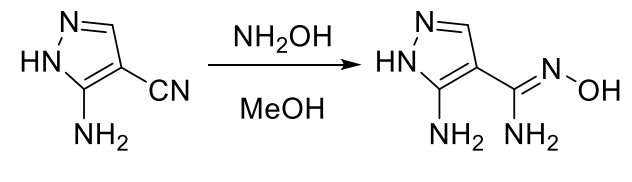

$1 \mathrm{i}$

$4 \mathbf{i}$

5-Amino- $N$ '-hydroxy-1H-pyrazole-4-carboximidamide (4i). White solid. Yield $1.18 \mathrm{~g}$ (84\%). mp 208-209 ${ }^{\circ} \mathrm{C}$ (dec.). ${ }^{1} \mathrm{H}$ NMR (400 MHz, DMSO-d 6 ): $\delta 11.60(\mathrm{~s}, 1 \mathrm{H}), 9.04(\mathrm{~s}, 1 \mathrm{H}), 7.73(\mathrm{~s}, 1 \mathrm{H}), 5.55(\mathrm{~s}, 2 \mathrm{H}), 5.10(\mathrm{~s}, 2 \mathrm{H}) .{ }^{13} \mathrm{C}$ NMR (101 MHz, DMSO-d $\left.d_{6}\right): \delta 152.8,148.9,126.9,99.4$. Anal. Calcd for $\mathrm{C}_{4} \mathrm{H}_{7} \mathrm{~N}_{5} \mathrm{O}: \mathrm{C}, 34.04 ; \mathrm{H}, 5.00 ; \mathrm{N}, 49.62$. Found: $\mathrm{C}, 34.08 ; \mathrm{H}, 5.02 ; \mathrm{N}, 49.68$.

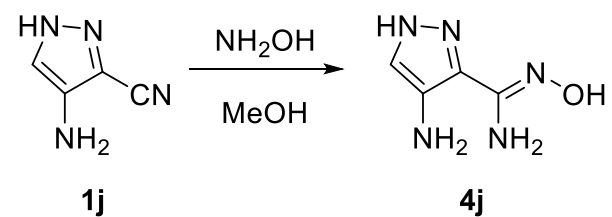

4-Amino- $N$ '-hydroxy- $1 \mathrm{H}$-pyrazole-3-carboximidamide (4j). White solid. Yield $1.23 \mathrm{~g}(87 \%) . \mathrm{mp} 212-213{ }^{\circ} \mathrm{C}$ (dec.). ${ }^{1} \mathrm{H}$ NMR (400 MHz, DMSO-d 6 ): $\delta 12.22(\mathrm{~s}, 1 \mathrm{H}), 9.36(\mathrm{~s}, 1 \mathrm{H}), 7.06(\mathrm{~s}, 1 \mathrm{H}), 5.42(\mathrm{~s}, 2 \mathrm{H}), 4.37(\mathrm{~s}, 2 \mathrm{H}) .{ }^{13} \mathrm{C}$ NMR (101 MHz, DMSO-d $\left.\mathrm{d}_{6}\right): \delta 149.4,131.4,128.9,114.8$. HRMS (ESI) m/z: $[\mathrm{M}+\mathrm{H}]^{+}$Calcd for $\mathrm{C}_{4} \mathrm{H}_{8} \mathrm{~N}_{5} \mathrm{O}^{+}$ 142.0723; Found 142.0724. IR: v 3445, 3345, 3281, 2797, 1651, 1603, 1570, 1538, 1477, 1385, 1342, 1326, $1202,1120,1080,957,927,827,731,694,640,613,561 \mathrm{~cm}^{-1}$. Anal. Calcd for $\mathrm{C}_{4} \mathrm{H}_{7} \mathrm{~N}_{5} \mathrm{O}: \mathrm{C}, 34.04 ; \mathrm{H}, 5.00$; N, 49.62. Found: $C, 34.09 ; H, 4.96 ; N, 49.66$.

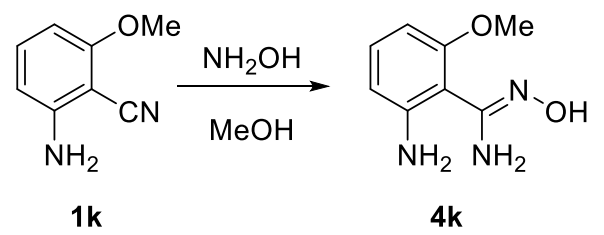


2-Amino- $N$ '-hydroxy-6-methoxybenzimidamide (4k). Yellow solid. Yield $1.61 \mathrm{~g}(89 \%) . \mathrm{mp} 119-120{ }^{\circ} \mathrm{C} .{ }^{1} \mathrm{H}$ NMR (400 MHz, DMSO-d 6 ): $\delta 9.64(\mathrm{~s}, 1 \mathrm{H}), 7.09$ (d, J = 8.0 Hz, 1H), $6.85(\mathrm{~d}, J=8.0 \mathrm{~Hz}, 1 \mathrm{H}), 6.58(\mathrm{t}, J=8.0$ $\mathrm{Hz}, 1 \mathrm{H}), 5.97(\mathrm{~s}, 2 \mathrm{H}), 5.78(\mathrm{~s}, 2 \mathrm{H}), 3.84(\mathrm{~s}, 3 \mathrm{H}) .{ }^{13} \mathrm{C}$ NMR $\left(101 \mathrm{MHz}, \mathrm{DMSO}-\mathrm{d}_{6}\right): \delta 152.8,146.6,136.4,119.3$, 114.3, 114.0, 110.1, 55.5. IR: $v$ 3313, 3120, 1671, 1600, 1524, 1476, 1429, 1372, 1298, 1268, 1199, 1161, $1059,950,919,808,755,655,610 \mathrm{~cm}^{-1}$. Anal. Calcd for $\mathrm{C}_{8} \mathrm{H}_{11} \mathrm{~N}_{3} \mathrm{O}_{2}: \mathrm{C}, 53.03 ; \mathrm{H}, 6.12 ; \mathrm{N}, 23.19$. Found: $\mathrm{C}$, $52.84 ; \mathrm{H}, 6.08 ; \mathrm{N}, 23.01$.

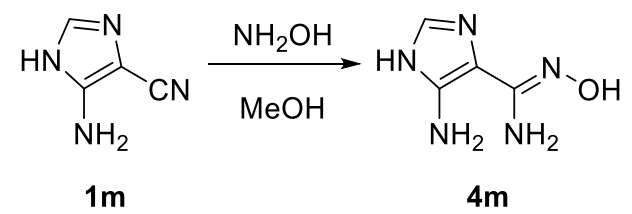

5-Amino- $N$ '-hydroxy-1H-imidazole-4-carboximidamide (4m). ${ }^{3}$ White solid. Yield $0.17 \mathrm{~g}$ (12\%). HRMS (ESI) $\mathrm{m} / \mathrm{z}:[\mathrm{M}+\mathrm{H}]^{+}$Calcd for $\mathrm{C}_{4} \mathrm{H}_{8} \mathrm{~N}_{5} \mathrm{O}^{+}$142.0723; Found 142.0734. [M + Na] $]^{+}$Calcd for $\mathrm{C}_{4} \mathrm{H}_{7} \mathrm{~N}_{5} \mathrm{NaO}^{+} 164.0543$; Found 164.0560.

\subsection{Synthesis of 1,2,3-triazine 3-oxide}

General procedure for the synthesis of $5 \mathrm{a}-5 \mathrm{~m}$ :

To a solution of $o$-aminoamidoxime $(4 \mathrm{a}-4 \mathrm{~m}, 5 \mathrm{mmol})$ in $6 \mathrm{M} \mathrm{HCl}(6 \mathrm{~mL})$ sodium nitrite $(0.41 \mathrm{~g}, 5.9 \mathrm{mmol})$ in $2 \mathrm{~mL}$ deionized water was added dropwise at $-5{ }^{\circ} \mathrm{C}$. The solution was stirred at $-5{ }^{\circ} \mathrm{C}$ for $30 \mathrm{~min}$. The precipitate was filtered off, washed with a small amount of water and dried in air to give the products.<smiles>N/C(=N\O)c1ccccc1NO[N+]([O-])([O-])[O-]</smiles>

4-Aminobenzo[1,2,3]triazine 3-oxide dihydrate (5a). White solid. Yield $0.71 \mathrm{~g}(72 \%)$. $\mathrm{mp} 174-175{ }^{\circ} \mathrm{C}$ (dec.). ${ }^{1} \mathrm{H}$ NMR (400 MHz, DMSO-d 6 ): $\delta 8.68(\mathrm{br}, 2 \mathrm{H}), 8.30(\mathrm{~d}, J=8.0 \mathrm{~Hz}, 1 \mathrm{H}), 8.18(\mathrm{~d}, J=8.0 \mathrm{~Hz}, 1 \mathrm{H}), 7.87(\mathrm{~m}, 2 \mathrm{H})$. ${ }^{13} \mathrm{C} \mathrm{NMR}\left(101 \mathrm{MHz}\right.$, DMSO-d 6 ): $\delta 141.4,138.4,131.8,131.8,128.2,121.7,110.3$. HRMS (ESI) m/z: $[\mathrm{M}+\mathrm{H}]^{+}$ Calcd for $\mathrm{C}_{7} \mathrm{H}_{7} \mathrm{~N}_{4} \mathrm{O}^{+}$163.0614; Found 163.0631. [M + Na] ${ }^{+}$Calcd for $\mathrm{C}_{7} \mathrm{H}_{6} \mathrm{~N}_{4} \mathrm{NaO}^{+}$185.0434; Found 185.0439. $[\mathrm{M}+\mathrm{K}]^{+}$Calcd for $\mathrm{C}_{7} \mathrm{H}_{6} \mathrm{KNN}_{4} \mathrm{O}^{+}$201.0173; Found 201.0175. [M - H] Calcd for $\mathrm{C}_{7} \mathrm{H}_{5} \mathrm{~N}_{4} \mathrm{O}^{-}$161.0469; Found 161.0487. IR: v 3494, 3337, 3220, 3066, 1670, 1628, 1583, 1518, 1467, 1446, 1372, 1281, 1244, 1209, $1166,1148,1118,1084,972,834,898,780,719,660,567 \mathrm{~cm}^{-1}$. Anal. Calcd for $\mathrm{C}_{7} \mathrm{H}_{10} \mathrm{~N}_{4} \mathrm{O}_{3}: \mathrm{C}, 42.42 ; \mathrm{H}$, 5.09; N, 28.27. Found: C, 42.49; H, 4.91; N, 28.01.<smiles>N/C(=N\O)c1cccnc1N</smiles>

4b 5b 
4-Aminopyrido[2,3- $d][1,2,3]$ triazine 3-oxide dihydrate (5b). Yellow solid. Yield $0.70 \mathrm{~g}(70 \%) . \mathrm{mp} 224-225^{\circ} \mathrm{C}$ (dec.). ${ }^{1} \mathrm{H}$ NMR (400 MHz, DMSO-d 6 ): $\delta 8.19(\mathrm{~m}, 1 \mathrm{H}), 7.85$ (d, J = 7.6 Hz, 1H), $6.88(\mathrm{~s}, 2 \mathrm{H}), 6.63$ (dd, $J_{1}=7.6$ $\left.\mathrm{Hz}, J_{2}=5.2 \mathrm{~Hz}, 1 \mathrm{H}\right) .{ }^{13} \mathrm{C}$ NMR $\left(101 \mathrm{MHz}, \mathrm{DMSO}-\mathrm{d}_{6}\right): \delta 160.0,153.2,142.3,117.0,111.9,89.2$. Anal. Calcd for $\mathrm{C}_{6} \mathrm{H}_{9} \mathrm{~N}_{5} \mathrm{O}_{3}: \mathrm{C}, 36.18 ; \mathrm{H}, 4.55 ; \mathrm{N}, 35.16$. Found: $\mathrm{C}, 36.04 ; \mathrm{H}, 4.51 ; \mathrm{N}, 35.08$.<smiles>N/C(=N\O)c1ccncc1N</smiles>

4c

5c

4-Aminopyrido[3,4-d][1,2,3]triazine 3-oxide (5c). White solid. Yield $0.55 \mathrm{~g}(67 \%) . \mathrm{mp} 170-178{ }^{\circ} \mathrm{C}$ (sub.). ${ }^{1} \mathrm{H}$ NMR $\left(400 \mathrm{MHz}, \mathrm{DMSO}-\mathrm{d}_{6}\right): \delta 9.53(\mathrm{~s}, 1 \mathrm{H}), 8.79(\mathrm{~s}, 1 \mathrm{H}) 8.15(\mathrm{~s}, 1 \mathrm{H}) .{ }^{13} \mathrm{C}$ NMR $\left(101 \mathrm{MHz}, \mathrm{DMSO}-\mathrm{d}_{6}\right): \delta 152.8$, 147.8, 140.6, 114.1, 113.9. Anal. Calcd for $\mathrm{C}_{6} \mathrm{H}_{9} \mathrm{~N}_{5} \mathrm{O}_{3}: \mathrm{C}, 36.18 ; \mathrm{H}, 4.55 ; \mathrm{N}, 35.16$. Found: $\mathrm{C}, 36.97 ; \mathrm{H}, 4.55$; N, 35.89 .

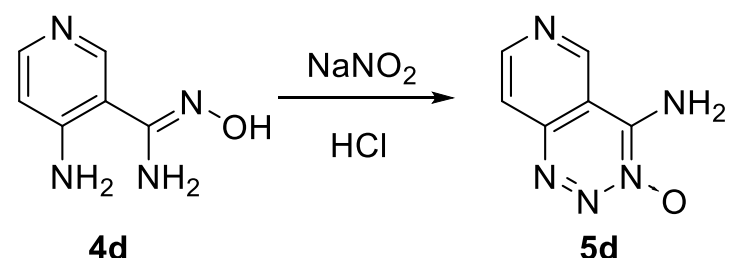

4-Aminopyrido[4,3-d][1,2,3]triazine 3-oxide (5d). White solid. Yield $0.53 \mathrm{~g}(65 \%) . \mathrm{mp} 53-55{ }^{\circ} \mathrm{C} .{ }^{1} \mathrm{H}$ NMR $\left(400 \mathrm{MHz}, \mathrm{DMSO}-\mathrm{d}_{6}\right): \delta 9.53(\mathrm{~s}, 1 \mathrm{H}), 8.90(\mathrm{br}, 2 \mathrm{H}), 8.79(\mathrm{~d}, J=5.6 \mathrm{~Hz}, 1 \mathrm{H}), 8.10(\mathrm{~d}, J=5.6 \mathrm{~Hz}, 1 \mathrm{H}) .{ }^{13} \mathrm{C} \mathrm{NMR}$ $\left(101 \mathrm{MHz}, \mathrm{DMSO}-\mathrm{d}_{6}\right): \delta 152.8,147.8,140.6,132.9,114.1,113.9$. Anal. Calcd for $\mathrm{C}_{6} \mathrm{H}_{9} \mathrm{~N}_{5} \mathrm{O}_{3}: \mathrm{C}, 36.18 ; \mathrm{H}$, 4.55; N, 35.16. Found: C, 36.04; H, 4.47; N, 35.24.

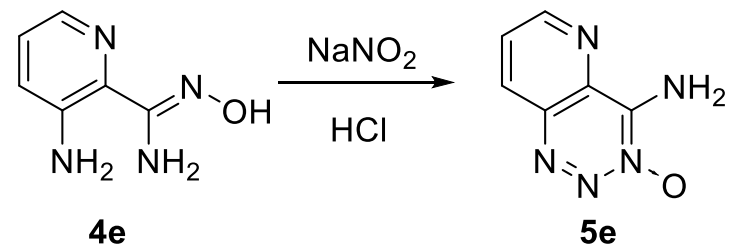

4-Aminopyrido[3,2-d][1,2,3]triazine 3-oxide (5e). Yellow solid. Yield $0.58 \mathrm{~g}(72 \%) . \mathrm{mp} 232-233{ }^{\circ} \mathrm{C}$ (dec.). ${ }^{1} \mathrm{H}$ NMR $\left(400 \mathrm{MHz}, \mathrm{DMSO}-\mathrm{d}_{6}\right): \delta 9.18(\mathrm{br}, 1 \mathrm{H}), 9.10(\mathrm{~d}, J=4.0 \mathrm{~Hz}, 1 \mathrm{H}), 8.60(\mathrm{~d}, J=8.6 \mathrm{~Hz}, 1 \mathrm{H}), 8.27(\mathrm{br}, 1 \mathrm{H})$, 7.90 (m, 1H). ${ }^{13} \mathrm{C}$ NMR (101 MHz, DMSO-d 6 ): $\delta$ 155.3, 143.1, 136.1, 134.4, 127.0, 126.4. HRMS (ESI) m/z: $[\mathrm{M}+\mathrm{Na}]^{+}$Calcd for $\mathrm{C}_{6} \mathrm{H}_{5} \mathrm{~N}_{5} \mathrm{NaO}^{+}$186.0386; Found 186.0390. IR: v 3243, 3154, 3066, 1679, 1588, 1549, 1450, 1420, 1316, 1246, 1232, 1201, 1191, 1113, 1047, 1035, 904, 818, 785, 656, 596, $571 \mathrm{~cm}^{-1}$. Anal. Calcd for $\mathrm{C}_{6} \mathrm{H}_{9} \mathrm{~N}_{5} \mathrm{O}_{3}: \mathrm{C}, 36.18 ; \mathrm{H}, 4.55 ; \mathrm{N}, 35.16$. Found: $\mathrm{C}, 36.12 ; \mathrm{H}, 4.20 ; \mathrm{N}, 35.00$.<smiles>CN(O[N+](=O)[O-])c1nccnc1/C(N)=N/O</smiles> 
3-Amino- $N$-hydroxypyrazine-2-carbimidoyl chloride (5f-1). White solid. Yield $0.59 \mathrm{~g} \mathrm{(72 \% ).} \mathrm{mp} 175-177^{\circ} \mathrm{C}$ (dec.). ${ }^{1} \mathrm{H}$ NMR $\left(400 \mathrm{MHz}, \mathrm{DMSO}-\mathrm{d}_{6}\right): \delta 12.73(\mathrm{~s}, 1 \mathrm{H}), 8.10(\mathrm{~d}, J=2.0 \mathrm{~Hz}, 1 \mathrm{H}), 7.97(\mathrm{~d}, J=2.0 \mathrm{~Hz}, 1 \mathrm{H}), 7.32$ (s, 2H). ${ }^{13} \mathrm{C}$ NMR $(101 \mathrm{MHz}$, DMSO-d $): \delta 152.7,143.2,138.4,131.9,127.2$. HRMS (ESI) m/z: [M+H] ${ }^{+}$Calcd for $\mathrm{C}_{6} \mathrm{H}_{6} \mathrm{CIN}_{4} \mathrm{O}^{+}$173.0225, 175.0195; Found 173.0224, 175.0196. IR: v 3373, 3280, 3148, 3017, 2865, 1632, $1564,1527,1492,1462,1440,1273,1237,1104,1038,966,664,818,786,686,554,512 \mathrm{~cm}^{-1}$. Anal. Calcd for $\mathrm{C}_{5} \mathrm{H}_{5} \mathrm{CIN}{ }_{4} \mathrm{O}: \mathrm{C}, 34.80 ; \mathrm{H}, 2.92 ; \mathrm{N}, 32.47$. Found: $\mathrm{C}, 34.21 ; \mathrm{H}, 3.03 ; \mathrm{N}, 31.74$.

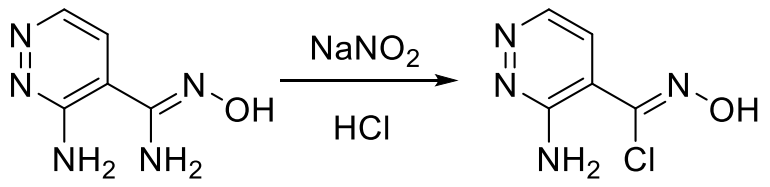

$4 g$

$5 g-1$

3-Amino- $N$-hydroxypyridazine-4-carbimidoyl chloride (5g-1). White solid. Yield $0.55 \mathrm{~g}(67 \%)$. mp 234$236{ }^{\circ} \mathrm{C}$. (dec.) ${ }^{1} \mathrm{H}$ NMR (400 MHz, DMSO-d 6 ): $\delta 13.84(\mathrm{~s}, 1 \mathrm{H}), 8.69(\mathrm{~d}, J=5.0 \mathrm{~Hz}, 1 \mathrm{H}), 8.64(\mathrm{br}, 2 \mathrm{H}), 8.24(\mathrm{~d}$, $J=5.0 \mathrm{~Hz}, 1 \mathrm{H}$ ). ${ }^{13} \mathrm{C}$ NMR $(101 \mathrm{MHz}$, DMSO-d 6 ): $\delta 153.4,141.4,132.7,131.7,123.0$. HRMS (ESI) m/z: [M + $\mathrm{H}]^{+}$Calcd for $\mathrm{C}_{5} \mathrm{H}_{6} \mathrm{ClN}_{4} \mathrm{O}^{+} 173.0225,175.0195$; Found 173.0236, 175.0089. IR: $v$ 3365, 3252, 3080, 2975, 2842, 1665, 1643, 1578, 1438, 1385, 1340, 1300, 1260, 1167, 1083, 1046, 973, 872, 833, 679, 663, 561 $\mathrm{cm}^{-1}$. Anal. Calcd for $\mathrm{C}_{5} \mathrm{H}_{5} \mathrm{ClN}{ }_{4} \mathrm{O}: \mathrm{C}, 34.80 ; \mathrm{H}, 2.92 ; \mathrm{N}, 32.47$. Found: $\mathrm{C}, 34.04 ; \mathrm{H}, 2.94 ; \mathrm{N}, 32.02$.<smiles>CN(O[N+](=O)[O-])c1ncncc1/C(N)=N/O</smiles>

4h

$5 h-1$

5-Amino- $N$-hydroxypyrimidine-4-carbimidoyl chloride (5h-1). White solid. Yield $0.62 \mathrm{~g} \mathrm{(76 \% ).} \mathrm{mp} \mathrm{213-}$ $214{ }^{\circ} \mathrm{C}$ (dec.). ${ }^{1} \mathrm{H}$ NMR (400 MHz, DMSO-d 6 ): $\delta 13.26$ (s, 1H), $9.86(\mathrm{~s}, 1 \mathrm{H}), 8.80(\mathrm{~s}, 1 \mathrm{H}), 8.74(\mathrm{~s}, 2 \mathrm{H}) .{ }^{13} \mathrm{C}$ NMR (101 MHz, DMSO-d $): \delta 160.5,151.1,145.2,131.3,108.7$. HRMS (ESI) m/z: $[\mathrm{M}+\mathrm{H}]^{+}$Calcd for $\mathrm{C}_{5} \mathrm{H}_{6} \mathrm{ClN}_{4} \mathrm{O}^{+}$ 173.0225, 175.0195; Found 173.0253, 175.0234. IR: $v$ 3336, 3204, 3002, 2892, 2855, 1664, 1626, 1572, $1527,1463,1384,1363,1302,1269,1210,1122,1038,958,860,836,780,690,582,523 \mathrm{~cm}^{-1}$. Anal. Calcd for $\mathrm{C}_{5} \mathrm{H}_{5} \mathrm{CIN}{ }_{4} \mathrm{O}: \mathrm{C}, 34.80 ; \mathrm{H}, 2.92 ; \mathrm{N}, 32.47$. Found: $\mathrm{C}, 34.10 ; \mathrm{H}, 3.12 ; \mathrm{N}, 31.85$.

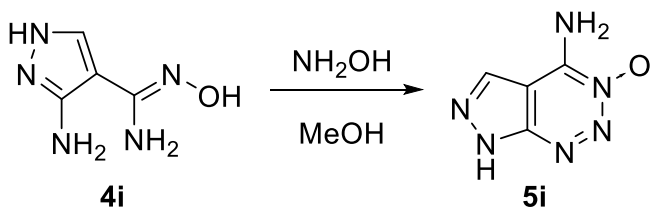

4-Amino-7H-pyrazolo[3,4- $d][1,2,3]$ triazine 3-oxide (5i). White solid. Yield $0.52 \mathrm{~g} \mathrm{(68 \% ).} \mathrm{mp} 212-213{ }^{\circ} \mathrm{C}$ (dec.). ${ }^{1} \mathrm{H}$ NMR (400 MHz, $\left.\mathrm{D}_{2} \mathrm{SO}_{4}\right): \delta 8.99$ (s, $\left.1 \mathrm{H}\right) .{ }^{13} \mathrm{C} \mathrm{NMR}\left(101 \mathrm{MHz}, \mathrm{D}_{2} \mathrm{SO}_{4}\right): \delta 150.3,137.5,122.3,110.9$. HRMS (ESI) m/z: [M-H] Calcd for $\mathrm{C}_{4} \mathrm{H}_{3} \mathrm{~N}_{6} \mathrm{O}^{-}$151.0374; Found 151.0356. Anal. Calcd for $\mathrm{C}_{4} \mathrm{H}_{4} \mathrm{~N}_{6} \mathrm{O}: \mathrm{C}, 31.58$; $H, 2.65 ; N, 55.25$. Found: C, 31.61; H, 2.68; N, 55.20. 


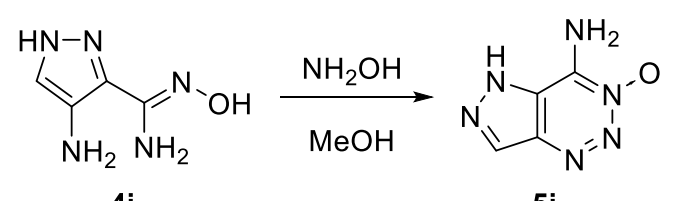

$4 \mathbf{j}$

$5 \mathbf{j}$

4-Amino-5H-pyrazolo[4,3-d][1,2,3]triazine 3-oxide (5j). White solid. Yield 0.54 g (72\%). $\mathrm{mp} 185.3-185.4{ }^{\circ} \mathrm{C}$ (dec.). ${ }^{1} \mathrm{H}$ NMR $\left(400 \mathrm{MHz}, \mathrm{D}_{2} \mathrm{SO}_{4}\right): \delta .9 .71(\mathrm{~s}, 1 \mathrm{H}) .{ }^{13} \mathrm{C} \mathrm{NMR}\left(101 \mathrm{MHz}, \mathrm{D}_{2} \mathrm{SO}_{4}\right): \delta 150.1,145.5,138.0,93.0$. HRMS (ESI) m/z: $[\mathrm{M}+\mathrm{H}]^{+}$Calcd for $\mathrm{C}_{4} \mathrm{H}_{5} \mathrm{~N}_{6} \mathrm{O}^{+}$153.0519; Found 153.0519. IR: $v$ 3351, 3114, 1985, 1697, 1592, 1556, 1506, 1428, 1337, 1287, 1206, 1178, 1072, 1047, 919, 901, 840, 679, 600, 587, $540 \mathrm{~cm}^{-1}$. Anal. Calcd for $\mathrm{C}_{4} \mathrm{H}_{4} \mathrm{~N}_{6} \mathrm{O}: \mathrm{C}, 31.58 ; \mathrm{H}, 2.65 ; \mathrm{N}, 55.25$. Found: $\mathrm{C}, 31.54 ; \mathrm{H}, 2.60 ; \mathrm{N}, 55.21$.<smiles>COc1cccc(N)c1/C(N)=N/O[N+]([O-])([O-])O</smiles>

4-Amino-5-methoxybenzo[1,2,3]triazine 3-oxide (5k). White solid. Yield $0.76 \mathrm{~g}(79 \%) . \mathrm{mp} 180-181{ }^{\circ} \mathrm{C} .{ }^{1} \mathrm{H}$ NMR (400 MHz, DMSO-d $): \delta 8.61(\mathrm{br}, 2 \mathrm{H}), 7.78(\mathrm{~m}, 2 \mathrm{H}), 7.35(\mathrm{t}, J=4.4 \mathrm{~Hz}, 1 \mathrm{H}), 4.03(\mathrm{~s}, 3 \mathrm{H}) .{ }^{13} \mathrm{C}$ NMR $(101$ MHz, DMSO-d $\left.\mathrm{d}_{6}\right): \delta 155.4,141.3,133.4,130.4,122.5,111.7,111.4,56.3$. HRMS (ESI) $\mathrm{m} / \mathrm{z}:[\mathrm{M}+\mathrm{H}]^{+}$Calcd for $\mathrm{C}_{8} \mathrm{H}_{9} \mathrm{~N}_{4} \mathrm{O}_{2}{ }^{+}$193.0720; Found 193.0724. [M + Na] ${ }^{+}$Calcd for $\mathrm{C}_{8} \mathrm{H}_{8} \mathrm{~N}_{4} \mathrm{NaO}_{2}{ }^{+}$215.0539; Found 215.0550. [M + $\mathrm{K}]^{+}$Calcd for $\mathrm{C}_{8} \mathrm{H}_{8} \mathrm{KNN}_{4} \mathrm{O}_{2}{ }^{+}$231.0279; Found 231.0260. IR: $v$ 3312, 1671, 1600, 1524, 1476, 1429, 1372, 1268, 1199, 1161, 1058, 950, 919, 808, 765, 665, $610 \mathrm{~cm}^{-1}$. Anal. Calcd for $\mathrm{C}_{8} \mathrm{H}_{8} \mathrm{~N}_{4} \mathrm{O}_{2}: \mathrm{C}, 50.00 ; \mathrm{H}, 4.20 ; \mathrm{N}, 29.15$. Found: C, 49.77; H, 4.44; N, 28.82.<smiles>N/C(=N\O)c1[nH]cnc1N</smiles>

4-Amino-5H-imidazo[4,5-d][1,2,3]triazine 3-oxide $(5 \mathrm{~m}) .{ }^{3}$ White solid. Yield $0.52 \mathrm{~g}(68 \%)$. Anal. Calcd for $\mathrm{C}_{4} \mathrm{H}_{4} \mathrm{~N}_{6} \mathrm{O}: \mathrm{C}, 31.58 ; \mathrm{H}, 2.65 ; \mathrm{N}, 55.25$. Found: C, 31.24; $\mathrm{H}, 2.76 ; \mathrm{N}, 55.20$. 


\section{Calculation Details}

All the DFT calculations were performed with Gaussian 09 suite of package. ${ }^{4}$ The geometry optimizations of all species in this study were performed using M06-2X method. The $6-31+G(d, p)$ basis set was used for all atoms. Frequency calculations at the same level of theory were carried out to characterize each stationary point (minimum or transition state) and to obtain the thermodynamic corrections to Gibbs free energy. Intrinsic reaction coordinate (IRC) ${ }^{5,6}$ was calculated to confirm the connection between the transition state and the right reactant/product. Then the single-point calculation was conducted to further refine the relative energies at $M P 2 / 6-311++G(d, p)$ level. The reported Gibbs free energy was calculated in the gas phase at $298.15 \mathrm{~K}, 1 \mathrm{~atm}$.

Table S1. Gibbs free energy change calculated under MP2/6-311++G(d, p) // M06-2X/6-31+G(d, p).

$\begin{array}{lllll} & \mathrm{E}\left(\mathrm{MP} 2 / 6-311++\mathrm{G}^{* *}\right) & \mathrm{TCG} & \mathrm{G} & \Delta \mathrm{G}(\mathrm{kcal} / \mathrm{mol}) \\ \text { IM-1 } & & & -839.582 & 0 \\ \text { TS-1 } & -763.347 & 0.098629 & -839.519 & 39.71 \\ \text { IM-2 } & -763.358 & 0.101349 & -839.527 & 34.85 \\ \text { IM-3 } & -687.12 & 0.075269 & -839.586 & -2.18 \\ \text { 3b } & -687.131 & 0.072395 & -839.6 & -10.95\end{array}$

Table S2. M06-2X geometries for IM-1.

$\begin{array}{llll}\text { Atom } & x & y & z \\ \mathrm{O} & -3.17317900 & -1.01706700 & 0.00037500 \\ \mathrm{O} & -2.08164900 & -2.87975000 & -0.00040900 \\ \mathrm{~N} & -0.89705400 & -1.05151800 & -0.00000400 \\ \mathrm{~N} & -1.86552900 & 1.15548000 & -0.00010800 \\ \mathrm{~N} & 1.79140800 & 1.29813700 & 0.00009800 \\ \mathrm{~N} & -2.10986400 & -1.66827200 & 0.00027600 \\ \mathrm{~N} & 2.92883600 & 0.52540300 & 0.00003900 \\ \mathrm{~N} & 3.07977500 & -1.63800700 & -0.00015500 \\ \mathrm{~N} & 3.74891100 & -0.53747300 & 0.00033300 \\ \mathrm{C} & 0.49070600 & 0.86946600 & -0.00006400 \\ \mathrm{C} & -0.81922700 & 0.28359600 & -0.00009000 \\ \mathrm{C} & 1.69841500 & 0.04270800 & 0.00001200 \\ \mathrm{C} & -0.50441000 & 3.08581800 & -0.00010700 \\ \mathrm{H} & -2.77983100 & 0.69161800 & -0.00017000 \\ \mathrm{H} & 1.03133600 & -1.97138100 & 0.00009700 \\ \mathrm{H} & -0.40265000 & 4.16276000 & -0.00013400 \\ \mathrm{C} & 0.62431200 & 2.23996000 & -0.00007900 \\ \mathrm{H} & 1.62889700 & 2.65232500 & -0.00007600\end{array}$




$\begin{array}{llll}\mathrm{C} & -1.74050100 & 2.50602300 & -0.00011400 \\ \mathrm{H} & -2.67027600 & 3.06145900 & -0.00015100\end{array}$

Table S3. M06-2X geometries for TS-1.

$\begin{array}{llll}\text { Atom } & x & y & z \\ \mathrm{O} & 0.07711100 & 0.80261300 & -0.40415200 \\ \mathrm{O} & -0.49004000 & -0.28377500 & 1.40791600 \\ \mathrm{~N} & 2.03194000 & -0.17306100 & 0.91563600 \\ \mathrm{~N} & 0.16356800 & 0.38560300 & 0.69909900 \\ \mathrm{~N} & 4.06727600 & 0.02515100 & 0.14696800 \\ \mathrm{~N} & 2.49922000 & -1.38971700 & 0.68456200 \\ \mathrm{~N} & 3.72502900 & -1.26720800 & 0.25358000 \\ \mathrm{C} & 2.76058800 & 2.12247100 & 0.48082800 \\ \mathrm{C} & 1.59397600 & 2.63843500 & 1.12892900 \\ \mathrm{C} & 2.99431500 & 0.69265200 & 0.54447200 \\ \mathrm{C} & 3.17244200 & 4.29592200 & -0.51610700 \\ \mathrm{H} & 1.22493900 & 1.24870300 & 2.42198600 \\ \mathrm{H} & 3.76960100 & 4.95310400 & -1.13449400 \\ \mathrm{C} & 3.54125800 & 2.95738500 & -0.30057500 \\ \mathrm{H} & 4.42019000 & 2.53567800 & -0.78023300 \\ \mathrm{C} & 1.99367400 & 4.74764200 & 0.02647100 \\ \mathrm{H} & 1.60083200 & 5.74477400 & -0.12874900 \\ \mathrm{~N} & 0.74180100 & 1.98894000 & 1.91544500 \\ \mathrm{~N} & 1.25607200 & 3.92642500 & 0.80262900 \\ \mathrm{H} & 0.39758800 & 4.25170800 & 1.23869300\end{array}$

Table S4. M06-2X geometries for IM-2.

$\begin{array}{llll}\text { Atom } & x & y & z \\ \text { O } & -1.20445900 & -1.40928700 & 1.41060600 \\ \mathrm{~N} & -1.40851000 & 0.23957500 & -0.15969200 \\ \mathrm{~N} & -1.20210600 & -1.18728700 & 0.17680200 \\ \mathrm{~N} & -0.98350600 & 2.33418900 & -0.06695300 \\ \mathrm{~N} & -2.59181300 & 0.84561700 & -0.08474800 \\ \mathrm{~N} & 2.32045300 & 2.10252800 & -0.06414900 \\ \mathrm{C} & 0.95206600 & 0.68055200 & -0.06304800 \\ \mathrm{C} & 1.12830200 & -0.70033700 & -0.23749100 \\ \mathrm{C} & -0.41623500 & 1.14891900 & -0.09462200 \\ \mathrm{C} & 3.32031700 & 0.84675300 & 0.16760800 \\ \mathrm{H} & 0.20447600 & -2.49469700 & -0.34492300 \\ \mathrm{H} & 4.22965800 & 1.41314800 & 0.32865000 \\ \mathrm{C} & 2.07715700 & 1.46810400 & 0.15171100 \\ \mathrm{H} & 1.96338900 & 2.53747200 & 0.29911900 \\ \mathrm{C} & 3.37849100 & -0.53466000 & -0.01997900 \\ \mathrm{H} & 4.33278700 & -1.05365900 & -0.00652600 \\ \mathrm{~N} & 0.02120300 & -1.51542100 & -0.56709800\end{array}$




$\begin{array}{llll}\mathrm{N} & 2.30464200 & -1.30619200 & -0.21244500 \\ \mathrm{O} & -2.20238500 & -1.88961500 & -0.53834200 \\ \mathrm{H} & -2.85236000 & -2.05808000 & 0.16846900\end{array}$

Table S5. M06-2X geometries for IM-3.

$\begin{array}{llll}\text { Atom } & x & y & z \\ \mathrm{O} & 2.12212000 & 2.21151800 & -0.00007700 \\ \mathrm{~N} & 1.18407700 & 1.43947500 & 0.00004700 \\ \mathrm{~N} & 1.51275100 & 0.07388200 & 0.00000400 \\ \mathrm{~N} & -0.04570000 & 1.76968300 & 0.00001300 \\ \mathrm{~N} & 2.60979800 & -1.69037900 & 0.00013600 \\ \mathrm{~N} & 1.30675100 & -2.06343600 & -0.00003000 \\ \mathrm{~N} & 2.27874900 & 1.27837500 & 0.00000200 \\ \mathrm{~N} & 2.76416000 & -0.41698100 & -0.00010700 \\ \mathrm{C} & -0.76951100 & -0.59788900 & 0.00001400 \\ \mathrm{C} & -1.03460100 & 0.78521100 & 0.00001400 \\ \mathrm{C} & 0.61850900 & -0.94434800 & 0.00000700 \\ \mathrm{C} & -1.83354100 & -1.50498200 & 0.00000200 \\ \mathrm{C} & -3.11403300 & -0.98931500 & -0.00000300 \\ \mathrm{H} & -3.98311200 & -1.63651800 & -0.00001200 \\ \mathrm{H} & -1.63192300 & -2.57137600 & -0.00000300 \\ \mathrm{C} & -3.27651200 & 0.40860700 & -0.00000300 \\ \mathrm{H} & -4.27542200 & 0.83771000 & -0.00001000\end{array}$

Table S6. M06-2X geometries for $\mathbf{3 b}$.

$\begin{array}{llll}\text { Atom } & x & y & z \\ \mathrm{O} & -1.21495300 & 2.94808700 & -0.00026200 \\ \mathrm{~N} & -0.61115700 & 1.88480500 & -0.00023300 \\ \mathrm{~N} & -1.41035000 & 0.76587300 & -0.00007600 \\ \mathrm{~N} & 0.69215100 & 1.87906600 & -0.00030000 \\ \mathrm{~N} & -2.81785200 & -1.34668200 & 0.00026700 \\ \mathrm{~N} & -1.57874500 & -1.55536300 & 0.00026000 \\ \mathrm{~N} & 2.66367000 & 0.71002900 & -0.00022400 \\ \mathrm{~N} & -3.93417400 & -1.28650300 & 0.00021600 \\ \mathrm{C} & 0.60293700 & -0.54914000 & 0.00007600 \\ \mathrm{C} & 1.31308400 & 0.66618400 & -0.00014800 \\ \mathrm{C} & -0.81123600 & -0.39506000 & 0.00007500 \\ \mathrm{C} & 1.30173200 & -1.76907800 & 0.00024400 \\ \mathrm{C} & 2.67484800 & -1.71560400 & 0.00017500 \\ \mathrm{H} & 3.27971200 & -2.61458400 & 0.00029500 \\ \mathrm{H} & 0.75392900 & -2.70600000 & 0.00042000 \\ \mathrm{C} & 3.29808000 & -0.44383800 & -0.00006700 \\ \mathrm{H} & 4.38450300 & -0.38347100 & -0.00013400\end{array}$




\section{Crystallographic Data}

X-ray qualified crystals of $\mathbf{2 a - 1}, \mathbf{2} \mathbf{b} \cdot 3 \mathrm{H}_{2} \mathrm{O}, \mathbf{3 b}, \mathbf{3 b}-\mathbf{2}, \mathbf{3} \mathbf{d}-\mathbf{1} \cdot 1 / 2 \mathrm{H}_{2} \mathrm{O}, \mathbf{3 e}, \mathbf{3} \mathbf{f}-\mathbf{1} \mathrm{H}_{2} \mathrm{O}, \mathbf{3} \mathbf{h}-\mathbf{1} \cdot \mathrm{H}_{2} \mathrm{O}, \mathbf{4 a}, \mathbf{5 a} \cdot 2 \mathrm{H}_{2} \mathrm{O}$ were obtained by dissolving the corresponding powder samples in ethyl acetate and slow evaporation of solvent.

Single crystal X-ray diffraction data was collected on an Oxford Xcalibur diffratometer with MoK $\alpha$ or CuK $\alpha$ monochromated radiation ( $\lambda=0.71073$ or $1.54178 \AA$, respectively) at 170,173 or $295 \mathrm{~K}$. The crystal structures were solved by ShelXT using intrinsic phasing methods. ${ }^{7}$ The structures were refined by ShelXL using full-matrix least-squares methods based on $\mathrm{F}^{2} .^{8}$ The molecular graphics were demonstrated on Olex2 GUI. ${ }^{9}$ All non-hydrogen atoms were refined anisotropically. The positions of hydrogen atoms in $\mathbf{3 b}$ $\mathbf{2}$ were calculated based on a riding model. Other hydrogen atoms were located from difference Fourier maps. All hydrogen atoms were refined isotropically.

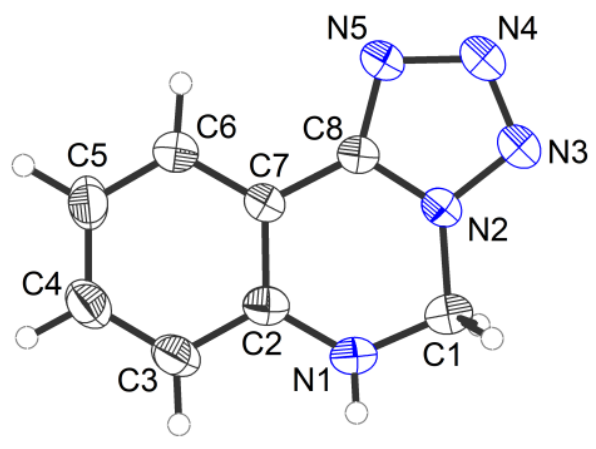

Figure S1. Crystal structure of 2a-1. Thermal ellipsoids were drawn at 50\% probability level.

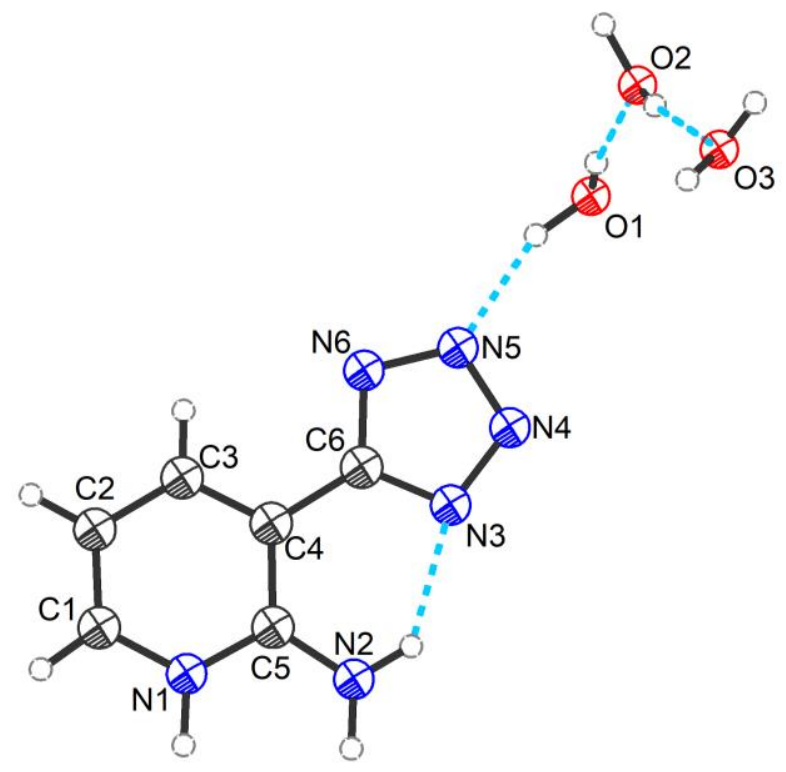

Figure S2. Crystal structure of $\mathbf{2 b} \cdot 3 \mathrm{H}_{2} \mathrm{O}$. Thermal ellipsoids were drawn at $50 \%$ probability level. Blue dotted lines represent hydrogen bonds. 


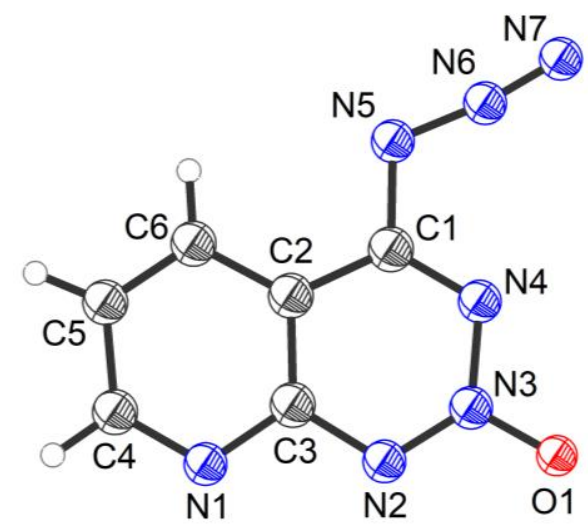

Figure S3. Crystal structure of 3b. Thermal ellipsoids were drawn at 50\% probability level.
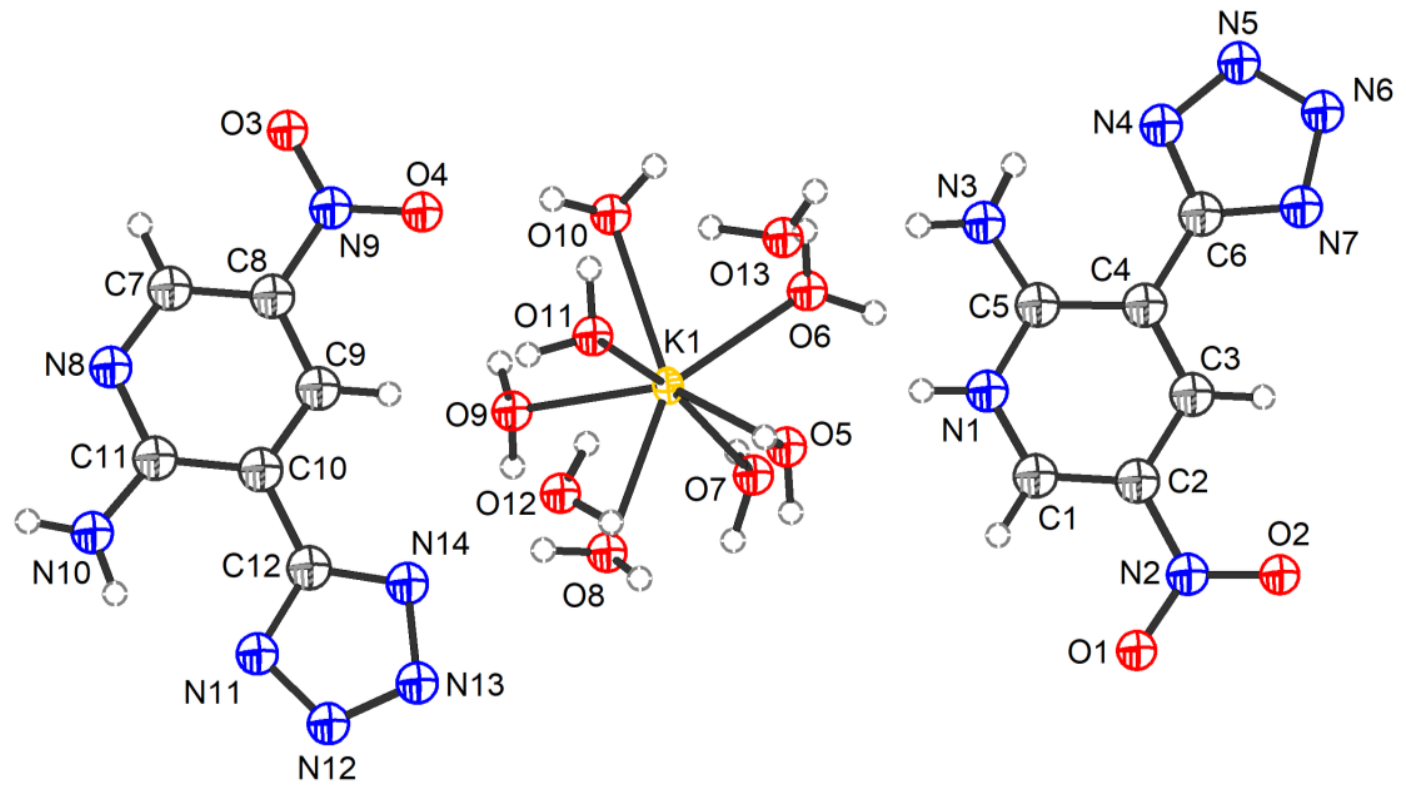

Figure S4. Crystal structure of $\mathbf{3 b - 2}$ (potassium salt with 9 hydrates). Thermal ellipsoids were drawn at $50 \%$ probability level.

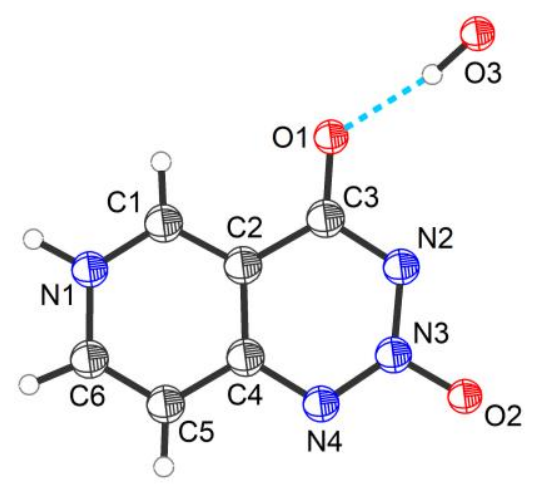

Figure S5. Crystal structure of $\mathbf{3 d}-\mathbf{1} \cdot \frac{1}{2} \mathrm{H}_{2} \mathrm{O}$. Thermal ellipsoids were drawn at $50 \%$ probability level. Blue dotted lines represent hydrogen bonds. 


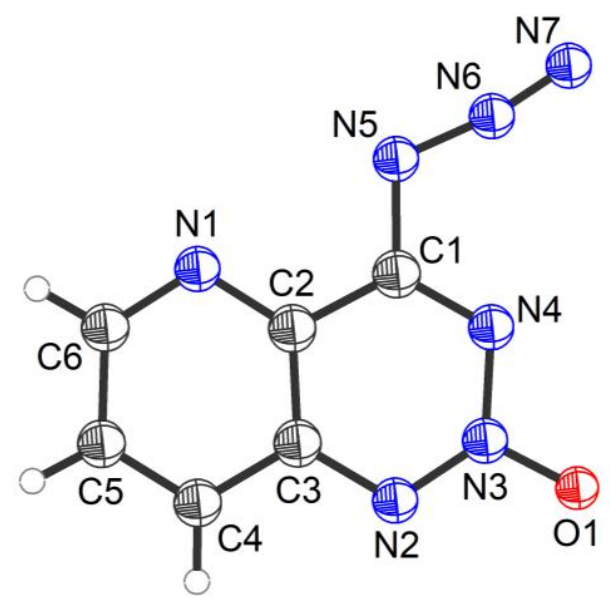

Figure S6. Crystal structure of 3e. Thermal ellipsoids were drawn at 50\% probability level.

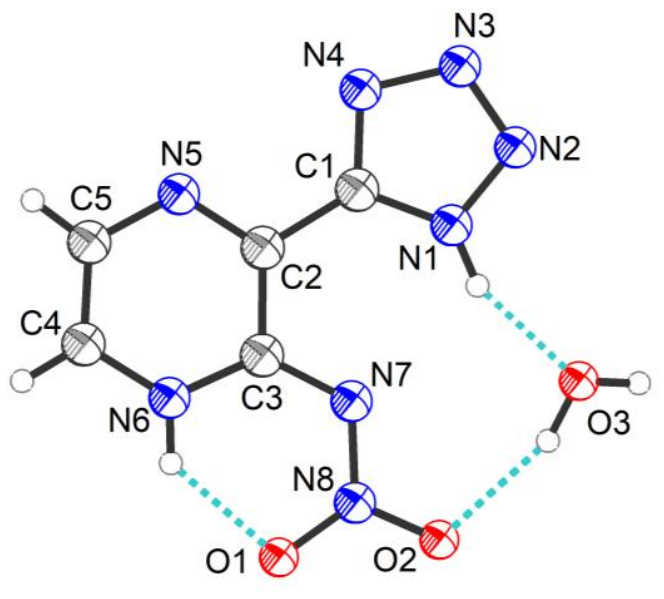

Figure S7. Crystal structure of $\mathbf{3 f - 1} \cdot \mathrm{H}_{2} \mathrm{O}$. Thermal ellipsoids were drawn at $50 \%$ probability level. Blue dotted lines represent hydrogen bonds.

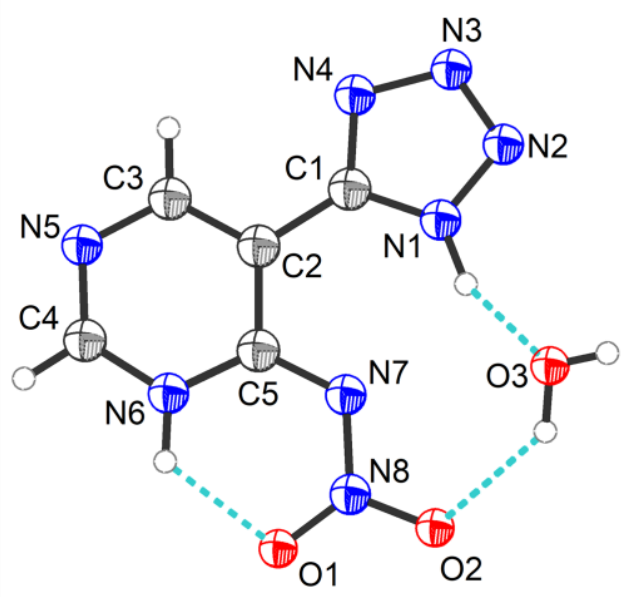

Figure S8. Crystal structure of $\mathbf{3 h}-\mathbf{1} \cdot \mathrm{H}_{2} \mathrm{O}$. Thermal ellipsoids were drawn at $\mathbf{5 0 \%}$ probability level. Another molecule of $\mathbf{3 h}-\mathbf{1} \cdot \mathrm{H}_{2} \mathrm{O}$ in the asymmetric unit was omitted for clarity. Blue dotted lines represent hydrogen bonds. 


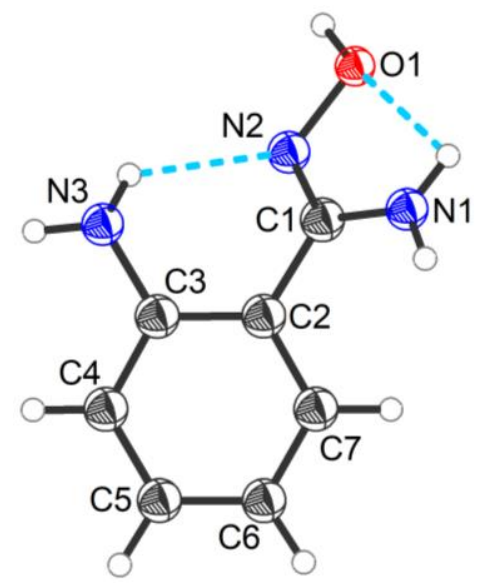

Figure S9. Crystal structure of 4a. Thermal ellipsoids were drawn at 50\% probability level. Other molecules of $\mathbf{4 a}$ in the asymmetric unit were omitted for clarity. Blue dotted lines represent hydrogen bonds.

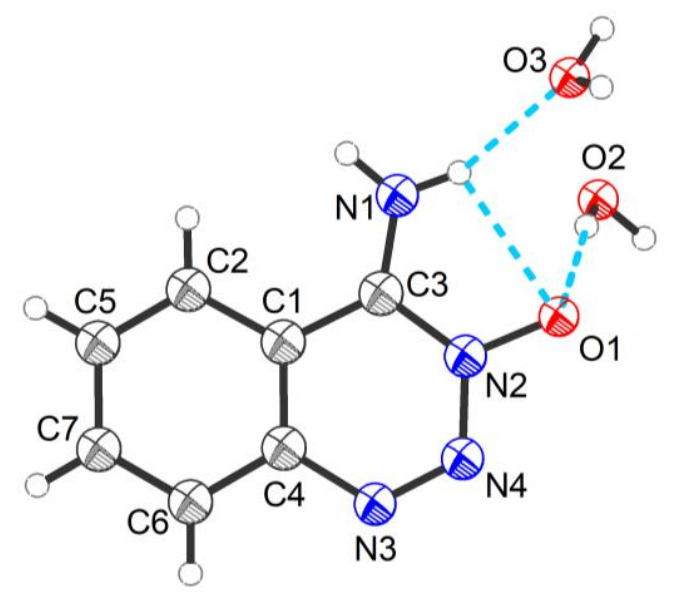

Figure S10. Crystal structure of $5 \mathrm{a} \cdot 2 \mathrm{H}_{2} \mathrm{O}$. Thermal ellipsoids were drawn at $50 \%$ probability level. Blue dotted lines represent hydrogen bonds. 
Table S7. Crystal data and structure refinement (1).

\begin{tabular}{|c|c|c|c|c|}
\hline Compound & $2 a-1$ & $\mathbf{2 b} \cdot 3 \mathrm{H}_{2} \mathrm{O}$ & $3 b$ & $3 b-2$ \\
\hline Empirical formula & $\mathrm{C}_{8} \mathrm{H}_{7} \mathrm{~N}_{5}$ & $\mathrm{C}_{6} \mathrm{H}_{12} \mathrm{~N}_{6} \mathrm{O}_{3}$ & $\mathrm{C}_{6} \mathrm{H}_{3} \mathrm{~N}_{7} \mathrm{O}$ & $\mathrm{C}_{12} \mathrm{H}_{27} \mathrm{KN}_{14} \mathrm{O}_{13}$ \\
\hline Formula weight & 173.19 & 216.22 & 189.15 & 614.57 \\
\hline Temperature/K & 173.0 & 170.0 & 170.0 & 170.0 \\
\hline Crystal system & triclinic & monoclinic & monoclinic & triclinic \\
\hline Space group & $P \overline{1}$ & $P 2_{1} / c$ & $P 2_{1} / c$ & $P \overline{1}$ \\
\hline $\mathrm{a} / \AA ̊ \AA$ & $6.8022(12)$ & $10.2202(4)$ & $9.8196(7)$ & $6.772(3)$ \\
\hline$b / \AA$ & $7.0362(15)$ & $15.3338(7)$ & 11.6192(7) & $12.173(5)$ \\
\hline$c / \AA ̊$ & 8.8798(16) & $6.7378(3)$ & $6.8836(4)$ & $15.622(6)$ \\
\hline$\alpha /^{\circ}$ & $74.652(7)$ & 90 & 90 & $95.721(12)$ \\
\hline$\beta /^{\circ}$ & 77.599(6) & $103.0683(18)$ & $103.218(2)$ & $91.037(11)$ \\
\hline$\gamma /{ }^{\circ}$ & $71.071(7)$ & 90 & 90 & $95.633(12)$ \\
\hline Volume $/ \AA^{3}$ & $383.79(13)$ & $1028.56(8)$ & $764.58(8)$ & $1274.8(9)$ \\
\hline Z & 2 & 4 & 4 & 2 \\
\hline$\rho_{\text {calc }} \mathrm{g} / \mathrm{cm}^{3}$ & 1.499 & 1.396 & 1.643 & 1.601 \\
\hline$\mu / \mathrm{mm}^{-1}$ & 0.101 & 0.113 & 0.126 & 0.298 \\
\hline$F(000)$ & 180.0 & 456.0 & 384.0 & 640.0 \\
\hline Crystal size $/ \mathrm{mm}^{3}$ & $0.26 \times 0.18 \times 0.12$ & $0.24 \times 0.19 \times 0.18$ & $0.24 \times 0.09 \times 0.08$ & $0.27 \times 0.09 \times 0.07$ \\
\hline Radiation & $\begin{array}{c}\text { MoK } \alpha \\
(\lambda=0.71073)\end{array}$ & $\begin{array}{c}\text { MoK } \alpha \\
(\lambda=0.71073)\end{array}$ & $\begin{array}{c}\text { MoK } \alpha \\
(\lambda=0.71073)\end{array}$ & $\begin{array}{c}\text { MoK } \alpha \\
(\lambda=0.71073)\end{array}$ \\
\hline $2 \Theta$ range for data collection ${ }^{\circ}$ & 4.806 to 52.73 & 4.878 to 52.804 & 7.014 to 52.776 & 5.244 to 50.054 \\
\hline Index ranges & $\begin{array}{l}-8 \leq h \leq 8 \\
-8 \leq k \leq 8 \\
-10 \leq 1 \leq 11\end{array}$ & $\begin{array}{c}-12 \leq \mathrm{h} \leq 12 \\
-18 \leq \mathrm{k} \leq 18 \\
-8 \leq \mathrm{I} \\
\leq 8\end{array}$ & $\begin{array}{c}-12 \leq \mathrm{h} \leq 11 \\
-14 \leq \mathrm{k} \leq 14 \\
-8 \leq \mathrm{I} \\
\leq 7\end{array}$ & $\begin{array}{c}-7 \leq h \leq 8 \\
-14 \leq k \leq 14 \\
-18 \leq \mathrm{I} \leq 18\end{array}$ \\
\hline Reflections collected & 3647 & 9489 & 5682 & 11804 \\
\hline Independent reflections & $\begin{array}{c}1533 \\
{\left[R_{\text {int }}=0.0581\right.} \\
\left.R_{\text {sigma }}=0.0740\right]\end{array}$ & $\begin{array}{c}2068 \\
{\left[R_{\text {int }}=0.0602,\right.} \\
\left.R_{\text {sigma }}=0.0513\right]\end{array}$ & $\begin{array}{c}1559 \\
{\left[R_{\text {int }}=0.0428\right.} \\
\left.R_{\text {sigma }}=0.0439\right]\end{array}$ & $\begin{array}{c}4459 \\
{\left[R_{\text {int }}=0.1290\right.} \\
\left.\mathrm{R}_{\text {sigma }}=0.1836\right]\end{array}$ \\
\hline Data/restraints/parameters & $1533 / 0 / 146$ & 2068/0/184 & 1559/0/139 & $4459 / 2 / 375$ \\
\hline Goodness-of-fit on $F^{2}$ & 1.062 & 1.076 & 1.088 & 1.066 \\
\hline Final $R$ indexes $[I>=2 \sigma(I)]$ & $\begin{array}{c}R_{1}=0.0801 \\
w R_{2}=0.1992\end{array}$ & $\begin{array}{c}R_{1}=0.0457 \\
w R_{2}=0.0893\end{array}$ & $\begin{array}{c}R_{1}=0.0420 \\
w R_{2}=0.0876\end{array}$ & $\begin{array}{c}R_{1}=0.1269 \\
W R_{2}=0.2692\end{array}$ \\
\hline Final $R$ indexes [all data] & $\begin{array}{r}R_{1}=0.1375 \\
W R_{2}=0.2441\end{array}$ & $\begin{array}{c}\mathrm{R}_{1}=0.0800 \\
\mathrm{wR}\end{array}$ & $\begin{array}{c}\mathrm{R}_{1}=0.0704 \\
\mathrm{wR}_{2}=0.1040\end{array}$ & $\begin{array}{c}\mathrm{R}_{1}=0.2082 \\
\mathrm{wR}_{2}=0.3061\end{array}$ \\
\hline Largest diff. peak/hole / e $\AA^{-3}$ & $0.37 /-0.23$ & $0.18 /-0.20$ & $0.19 /-0.23$ & $1.15 /-0.60$ \\
\hline CCDC Number & 2035798 & 2035803 & 2035802 & 2047071 \\
\hline
\end{tabular}


Table S8. Crystal data and structure refinement (2).

\begin{tabular}{|c|c|c|c|c|}
\hline Compound & 3d-1.1 $1 / 2 \mathrm{H}_{2} \mathrm{O}$ & $3 e$ & $3 f-1 \mathrm{H}_{2} \mathrm{O}$ & $3 h-1 \cdot \mathrm{H}_{2} \mathrm{O}$ \\
\hline Empirical formula & $\mathrm{C}_{6} \mathrm{H}_{5} \mathrm{~N}_{4} \mathrm{O}_{2.5}$ & $\mathrm{C}_{6} \mathrm{H}_{3} \mathrm{~N}_{7} \mathrm{O}$ & $\mathrm{C}_{5} \mathrm{H}_{6} \mathrm{~N}_{8} \mathrm{O}_{3}$ & $\mathrm{C}_{5} \mathrm{H}_{6} \mathrm{~N}_{8} \mathrm{O}_{3}$ \\
\hline Formula weight & 173.14 & 189.15 & 226.18 & 226.18 \\
\hline Temperature/K & 170.0 & 170.0 & 170.0 & 170.0 \\
\hline Crystal system & monoclinic & monoclinic & monoclinic & monoclinic \\
\hline Space group & $P 2 / C$ & $12 / a$ & $P 2_{1} / c$ & $P 2_{1} / C$ \\
\hline$a / \AA ̊$ & $9.3902(18)$ & $16.3690(7)$ & $6.7334(2)$ & $6.7568(5)$ \\
\hline $\mathrm{b} / \AA ̊ \AA$ & $4.5634(9)$ & $4.9203(2)$ & $15.5167(6)$ & $18.2448(14)$ \\
\hline$c / \AA ̊$ & $15.734(3)$ & $18.2740(7)$ & $8.6401(3)$ & $13.8771(10)$ \\
\hline$\alpha /^{\circ}$ & 90 & 90 & 90 & 90 \\
\hline$\beta /^{\circ}$ & $94.483(7)$ & $91.270(3)$ & $110.321(1)$ & $95.614(3)$ \\
\hline$\gamma /{ }^{\circ}$ & 90 & 90 & 90 & 90 \\
\hline Volume $/ \AA^{3}$ & $672.2(2)$ & $1471.43(10)$ & $846.54(5)$ & $1702.5(2)$ \\
\hline Z & 4 & 8 & 4 & 8 \\
\hline$\rho_{\text {calc }} \mathrm{g} / \mathrm{cm}^{3}$ & 1.711 & 1.708 & 1.775 & 1.765 \\
\hline$\mu / \mathrm{mm}^{-1}$ & 0.138 & 0.131 & 0.149 & 0.148 \\
\hline$F(000)$ & 356.0 & 768.0 & 464.0 & 928.0 \\
\hline Crystal size $/ \mathrm{mm}^{3}$ & $0.19 \times 0.08 \times 0.07$ & $0.23 \times 0.19 \times 0.16$ & $0.16 \times 0.12 \times 0.07$ & $0.19 \times 0.09 \times 0.07$ \\
\hline Radiation & $\begin{array}{c}\text { MoK } \alpha \\
(\lambda=0.71073)\end{array}$ & $\begin{array}{c}\text { MoK } \alpha \\
(\lambda=0.71073)\end{array}$ & $\begin{array}{c}\text { MoK } \alpha \\
(\lambda=0.71073)\end{array}$ & $\begin{array}{c}\text { MoK } \alpha \\
(\lambda=0.71073)\end{array}$ \\
\hline $2 \Theta$ range for data collection $/^{\circ}$ & 4.35 to 52.58 & \multicolumn{3}{|c|}{6.612 to 54.9965 .672 to 52.8084 .466 to 54.902} \\
\hline Index ranges & $\begin{array}{c}-11 \leq h \leq 11 \\
-5 \leq k \leq 5 \\
-19 \leq 1 \leq 19\end{array}$ & $\begin{array}{c}-21 \leq \mathrm{h} \leq 19 \\
-6 \leq \mathrm{k} \leq 6 \\
-23 \leq \mathrm{I} \leq 23\end{array}$ & $\begin{array}{r}-7 \leq h \leq 8 \\
-19 \leq k \leq 19 \\
-10 \leq I \leq 10\end{array}$ & $\begin{array}{r}-7 \leq h \leq 8 \\
-23 \leq k \leq 23 \\
-17 \leq I \leq 17\end{array}$ \\
\hline Reflections collected & 6481 & 8453 & 9368 & 20155 \\
\hline Independent reflections & $\begin{array}{c}1352 \\
{\left[R_{\text {int }}=0.0776\right.} \\
\left.R_{\text {sigma }}=0.0651\right]\end{array}$ & $\begin{array}{c}1689 \\
{\left[R_{\text {int }}=0.0459\right.} \\
\left.R_{\text {sigma }}=0.0364\right]\end{array}$ & $\begin{array}{c}1741 \\
{\left[R_{\text {int }}=0.0446,\right.} \\
\left.R_{\text {sigma }}=0.0323\right]\end{array}$ & $\begin{array}{c}3854 \\
{\left[R_{\text {int }}=0.0931,\right.} \\
\left.R_{\text {sigma }}=0.0755\right]\end{array}$ \\
\hline Data/restraints/parameters & $1352 / 0 / 135$ & $1689 / 7 / 127$ & $1741 / 0 / 169$ & $3854 / 0 / 337$ \\
\hline Goodness-of-fit on $\mathrm{F}^{2}$ & 1.073 & 1.041 & 1.107 & 1.026 \\
\hline Final $R$ indexes $[\mid>=2 \sigma(I)]$ & $\begin{array}{c}R_{1}=0.0561 \\
w R_{2}=0.1229\end{array}$ & $\begin{array}{c}R_{1}=0.0387 \\
w R_{2}=0.0931\end{array}$ & $\begin{array}{c}R_{1}=0.0419 \\
W R_{2}=0.0880\end{array}$ & $\begin{array}{c}\mathrm{R}_{1}=0.0548 \\
w \mathrm{R}_{2}=0.1064\end{array}$ \\
\hline Final $R$ indexes [all data] & $\begin{array}{c}R_{1}=0.1075 \\
w R_{2}=0.1523\end{array}$ & $\begin{array}{c}R_{1}=0.0549 \\
w R_{2}=0.1027\end{array}$ & $\begin{array}{c}\mathrm{R}_{1}=0.0579 \\
\mathrm{wR}_{2}=0.0971\end{array}$ & $\begin{array}{c}R_{1}=0.1162 \\
w R_{2}=0.1343\end{array}$ \\
\hline Largest diff. peak/hole / e $\AA^{-3}$ & $0.31 /-0.24$ & $0.21 /-0.22$ & $0.26 /-0.25$ & $0.28 /-0.31$ \\
\hline CCDC Number & 2035807 & 2035805 & 2035804 & 2035800 \\
\hline
\end{tabular}


Table S9. Crystal data and structure refinement (3).

\begin{tabular}{|c|c|c|}
\hline Compound & $4 a$ & $5 \mathrm{a} \cdot 2 \mathrm{H}_{2} \mathrm{O}$ \\
\hline Empirical formula & $\mathrm{C}_{7} \mathrm{H}_{9} \mathrm{~N}_{3} \mathrm{O}$ & $\mathrm{C}_{7} \mathrm{H}_{10} \mathrm{~N}_{4} \mathrm{O}_{3}$ \\
\hline Formula weight & 151.17 & 198.19 \\
\hline Temperature/K & 170.0 & 294.72 \\
\hline Crystal system & triclinic & monoclinic \\
\hline Space group & $P \overline{1}$ & $P 2_{1} / n$ \\
\hline $\mathrm{a} / \AA ̊ \AA$ & $7.0345(3)$ & $7.3450(2)$ \\
\hline b/Å & $9.7718(4)$ & $7.2749(13)$ \\
\hline$c / \AA ̊$ & $23.3724(9)$ & $16.8400(2)$ \\
\hline$\alpha /^{\circ}$ & $79.3216(13)$ & 90 \\
\hline$\beta /{ }^{\circ}$ & $87.7578(13)$ & $101.920(6)$ \\
\hline$\gamma / /^{\circ}$ & $76.0282(14)$ & 90 \\
\hline Volume $/ \AA^{3}$ & $1532.05(11)$ & $880.50(11)$ \\
\hline Z & 8 & 4 \\
\hline$\rho_{\text {calc }} \mathrm{g} / \mathrm{cm}^{3}$ & 1.311 & 1.495 \\
\hline$\mu / \mathrm{mm}^{-1}$ & 0.093 & 1.018 \\
\hline$F(000)$ & 640.0 & 416.0 \\
\hline Crystal size $/ \mathrm{mm}^{3}$ & $0.27 \times 0.18 \times 0.16$ & $0.2 \times 0.16 \times 0.14$ \\
\hline Radiation & $\begin{array}{c}\text { MoK } \alpha \\
(\lambda=0.71073)\end{array}$ & $\begin{array}{c}\text { CuK } \alpha \\
(\lambda=1.54178)\end{array}$ \\
\hline $2 \Theta$ range for data collection $/^{\circ}$ & 4.368 to 52.852 & $\begin{array}{c}10.738 \text { to } \\
148.938\end{array}$ \\
\hline Index ranges & $\begin{array}{c}-8 \leq h \leq 8 \\
-12 \leq k \leq 12 \\
-29 \leq 1 \leq 29\end{array}$ & $\begin{array}{l}-9 \leq h \leq 8 \\
-9 \leq k \leq 9 \\
-21 \leq 1 \leq 20\end{array}$ \\
\hline Reflections collected & 17449 & 14455 \\
\hline Independent reflections & $\begin{array}{c}6217 \\
{\left[R_{\text {int }}=0.0511\right.} \\
\left.R_{\text {sigma }}=0.0576\right]\end{array}$ & $\begin{array}{c}1779 \\
{\left[R_{\text {int }}=0.2288,\right.} \\
\left.R_{\text {sigma }}=0.0550\right]\end{array}$ \\
\hline Data/restraints/parameters & $6217 / 0 / 541$ & $1779 / 0 / 134$ \\
\hline Goodness-of-fit on $\mathrm{F}^{2}$ & 1.032 & 1.154 \\
\hline Final $R$ indexes $[\mid>=2 \sigma(I)]$ & $\begin{array}{c}R_{1}=0.0448 \\
w R_{2}=0.0997\end{array}$ & $\begin{array}{c}R_{1}=0.0713 \\
w R_{2}=0.1801\end{array}$ \\
\hline Final $R$ indexes [all data] & $\begin{array}{c}R_{1}=0.0647 \\
w R_{2}=0.1127\end{array}$ & $\begin{array}{c}R_{1}=0.0899 \\
w R_{2}=0.2187\end{array}$ \\
\hline Largest diff. peak/hole / e $\AA^{-3}$ & $0.21 /-0.25$ & $0.28 /-0.39$ \\
\hline CCDC Number & 2035799 & 2035806 \\
\hline
\end{tabular}


4. NMR Spectra 


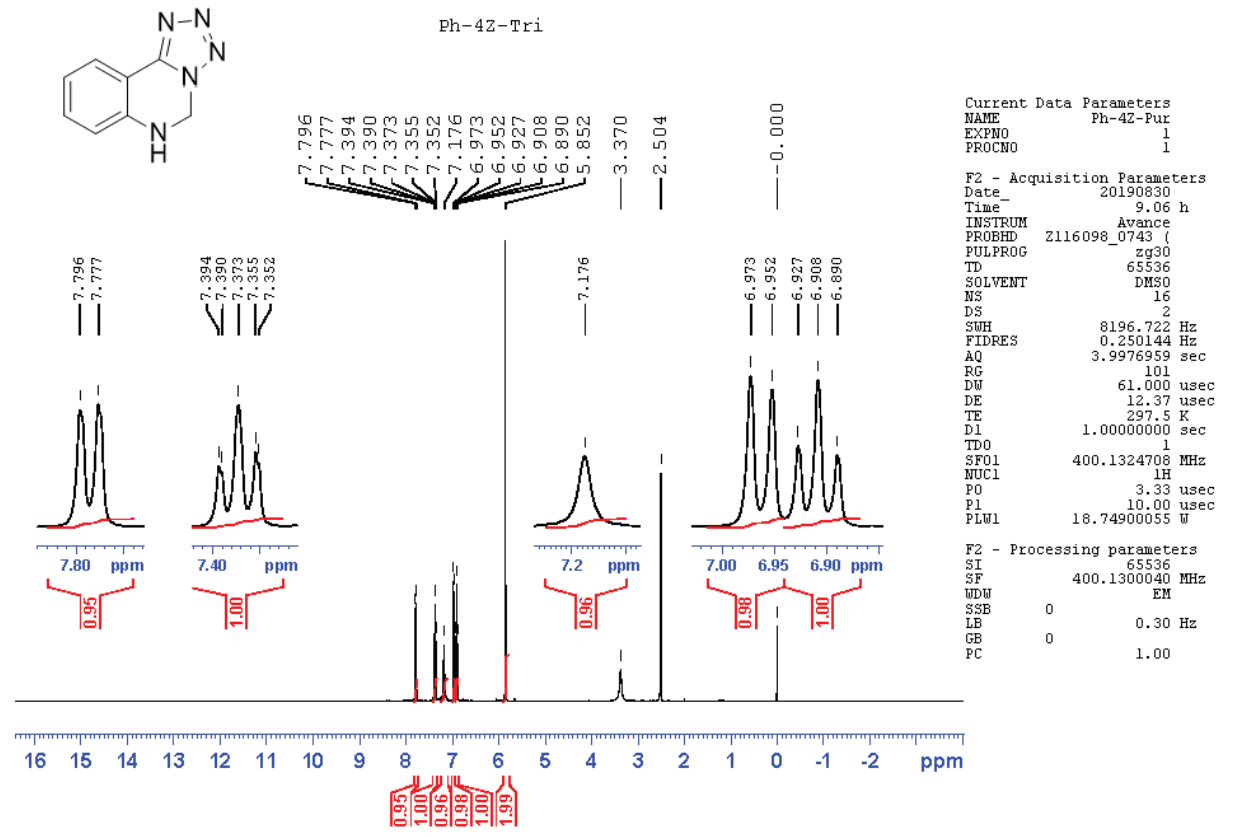

Figure S11. ${ }^{1} \mathrm{H}$ NMR of $2 \mathrm{a}-\mathbf{1}$ in DMSO- $\mathrm{d}_{6}$ operating at $400.13 \mathrm{MHz}$.

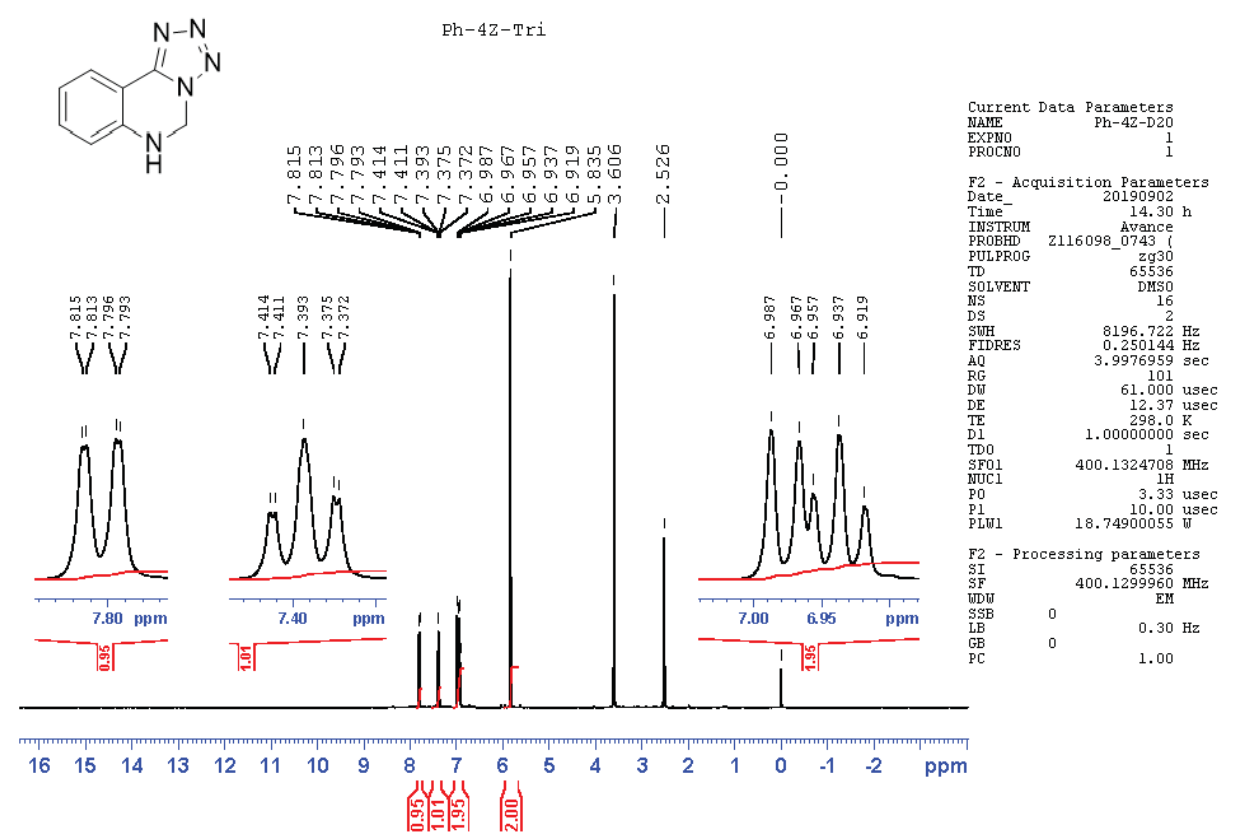

Figure S12. ${ }^{1} \mathrm{H}$ NMR of $\mathbf{2 a - 1}$ (with a drop of $\mathrm{D}_{2} \mathrm{O}$ ) in DMSO-d $\mathrm{d}_{6}$ operating at $400.13 \mathrm{MHz}$. 


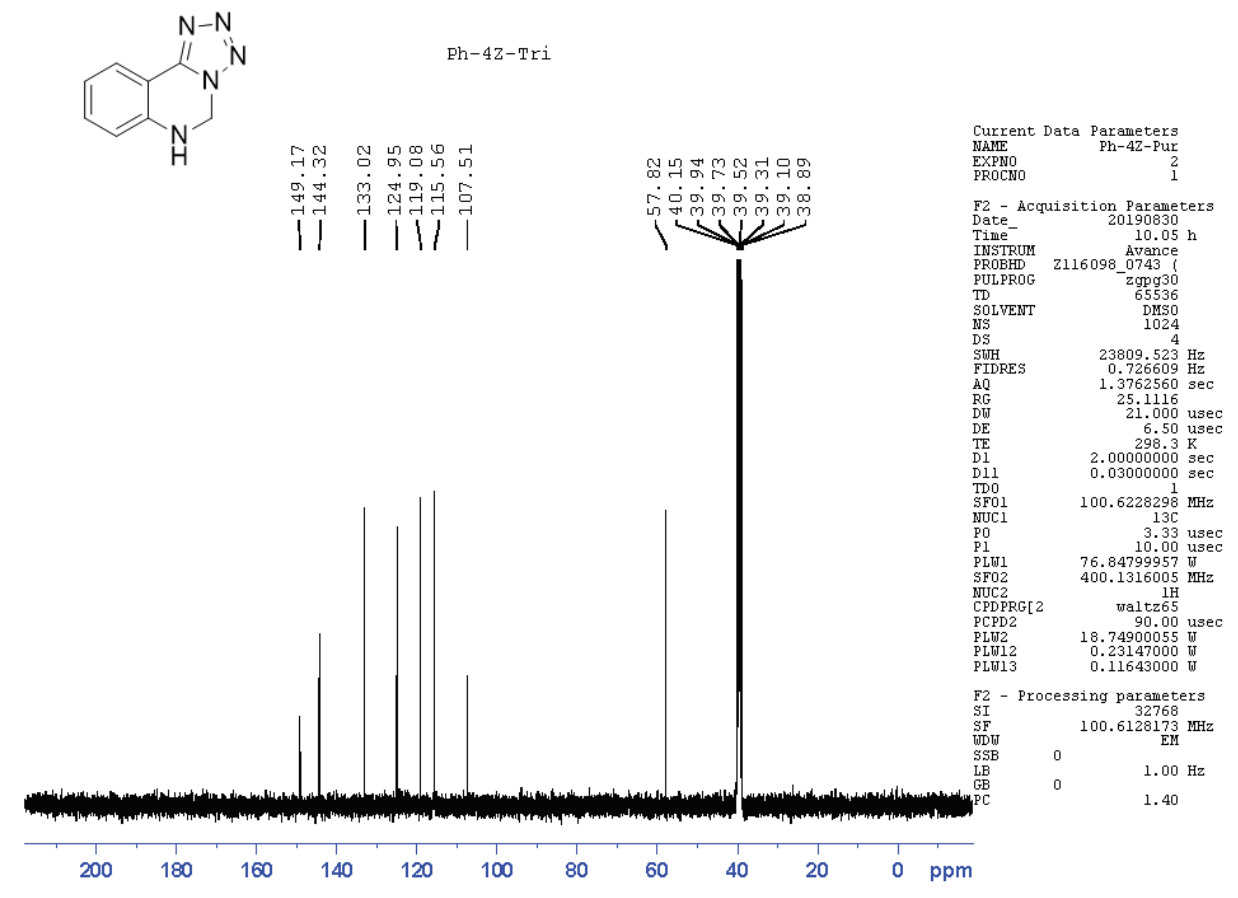

Figure S13. ${ }^{13} \mathrm{C}$ NMR of $2 \mathrm{a}-\mathbf{1}$ in DMSO-d $\mathrm{d}_{6}$ operating at $100.61 \mathrm{MHz}$.

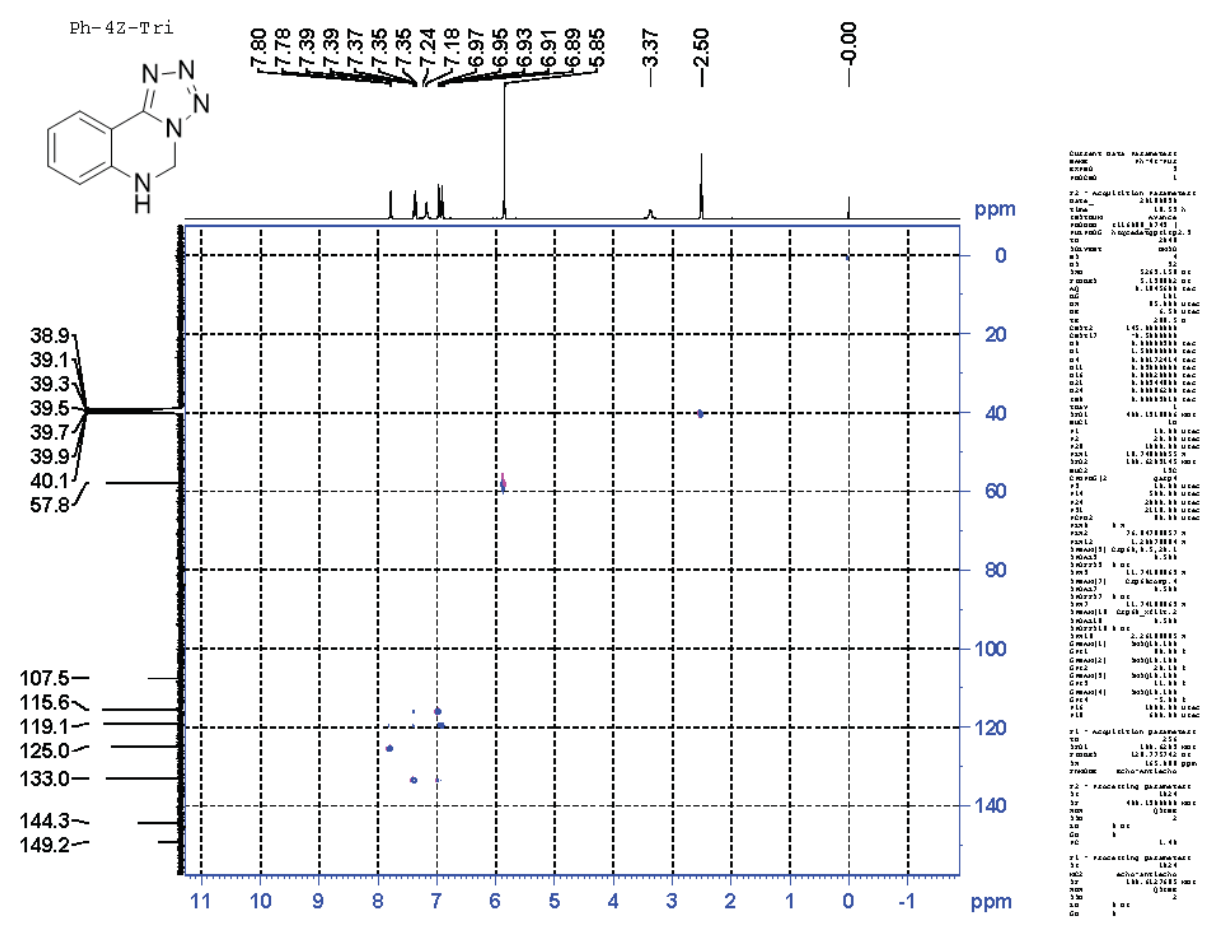

Figure S14. ${ }^{1} \mathrm{H}-{ }^{13} \mathrm{C}$ HSQC of $2 \mathrm{a}-1$ in DMSO- $\mathrm{d}_{6}$ operating at $100.61 \mathrm{MHz}$ (F1) / $400.13 \mathrm{MHz}$ (F2). 


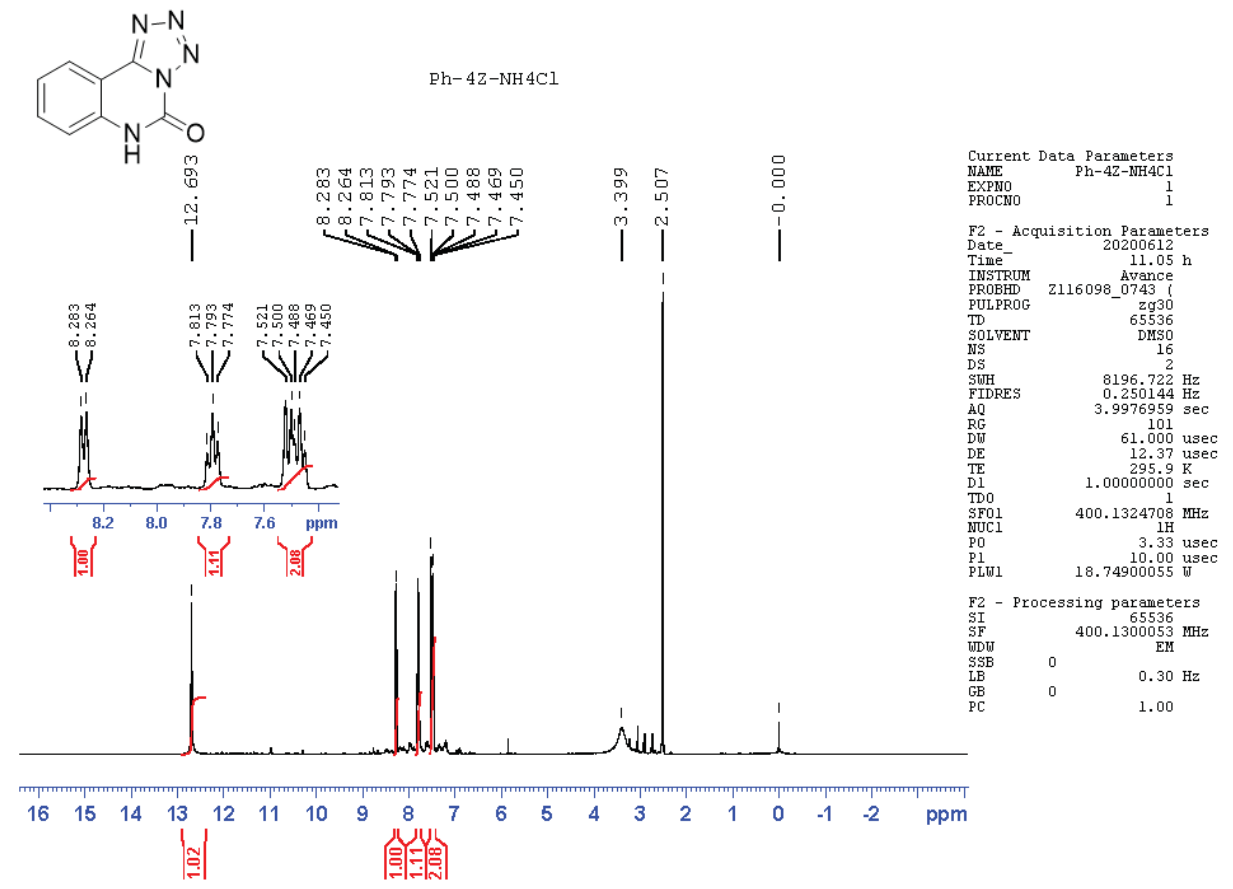

Figure S15. ${ }^{1} \mathrm{H}$ NMR of $\mathbf{2 a - 2}$ in DMSO- $\mathrm{d}_{6}$ operating at $400.13 \mathrm{MHz}$.

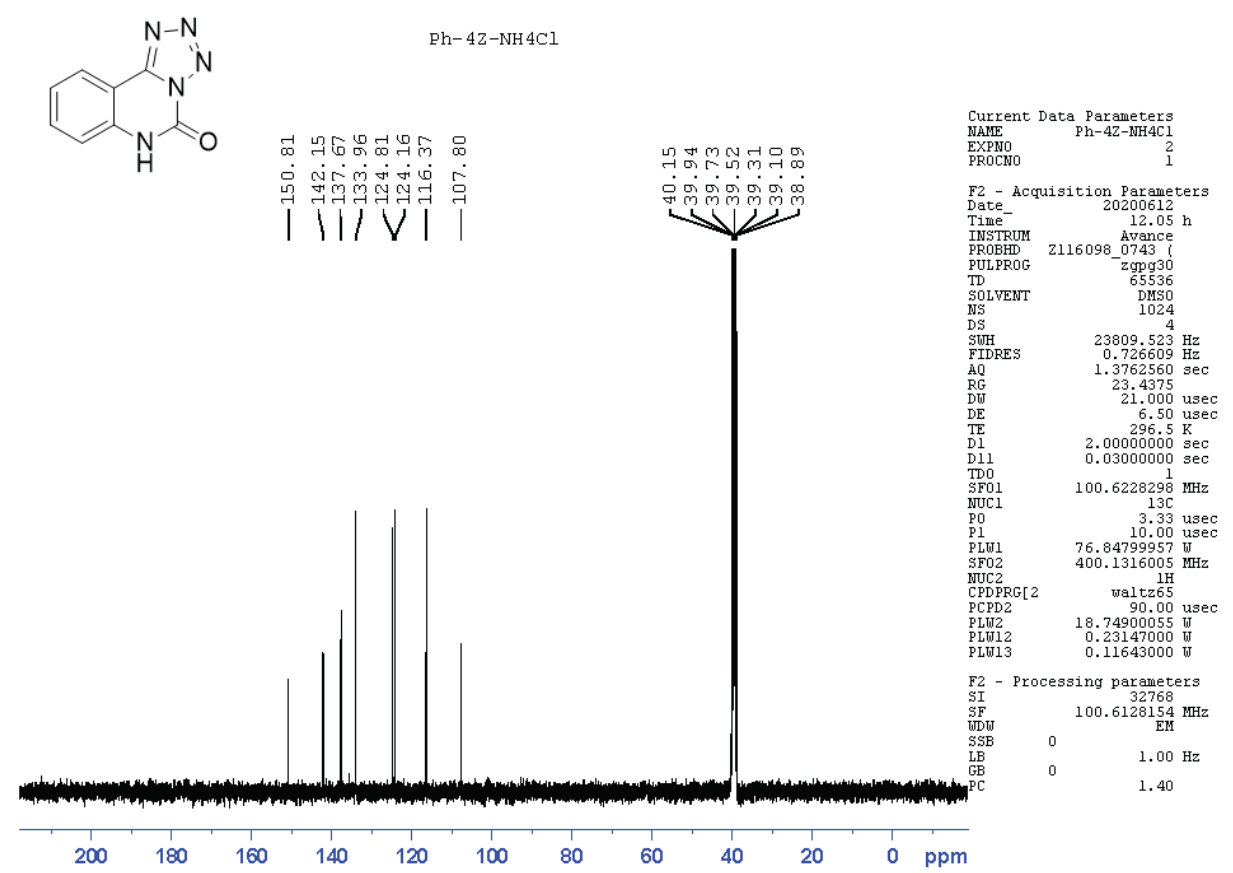

Figure S16. ${ }^{13} \mathrm{C}$ NMR of $\mathbf{2 a - 2}$ in DMSO-d $\mathrm{d}_{6}$ operating at $100.61 \mathrm{MHz}$. 


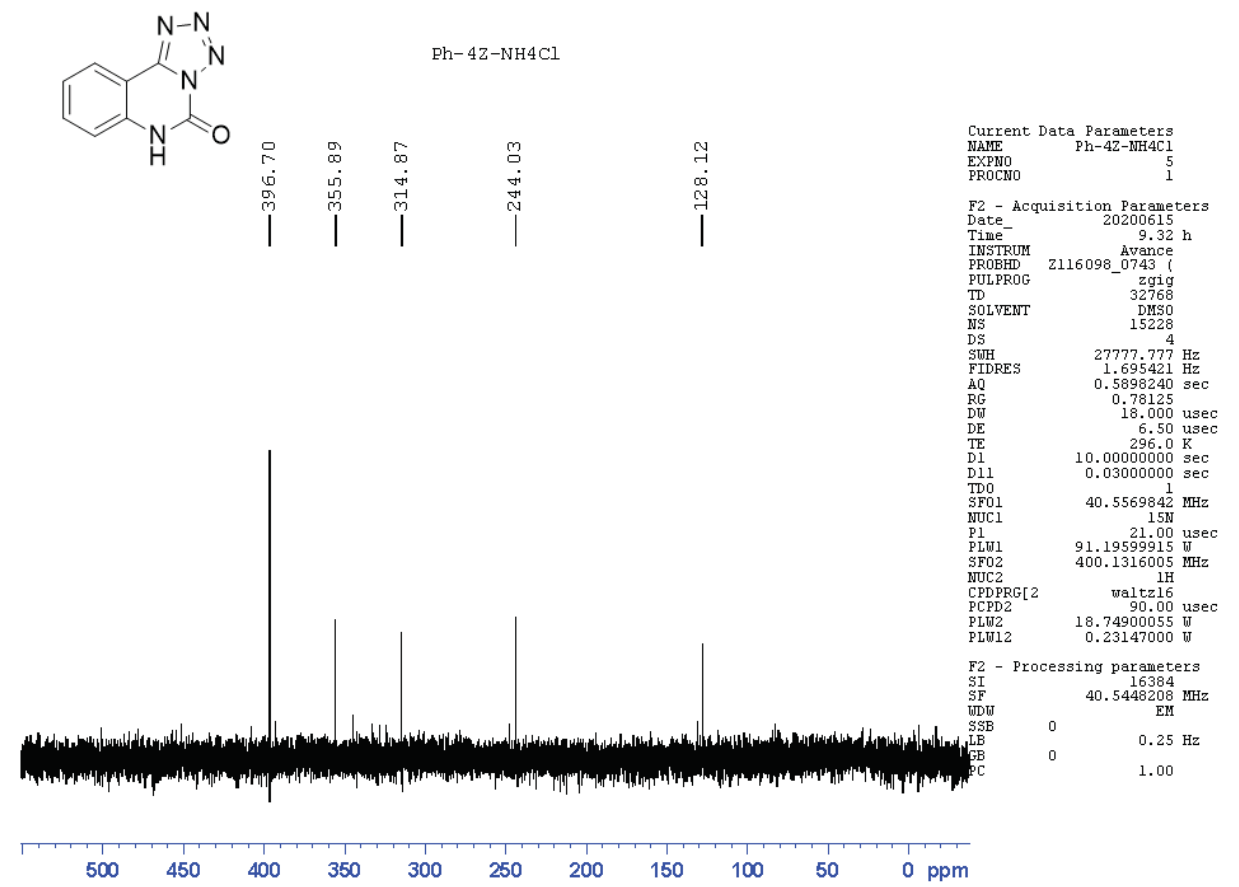

Figure S17. ${ }^{15} \mathrm{~N}$ NMR of $\mathbf{2 a - 2}$ in DMSO-d $\mathrm{d}_{6}$ operating at $40.54 \mathrm{MHz}$.

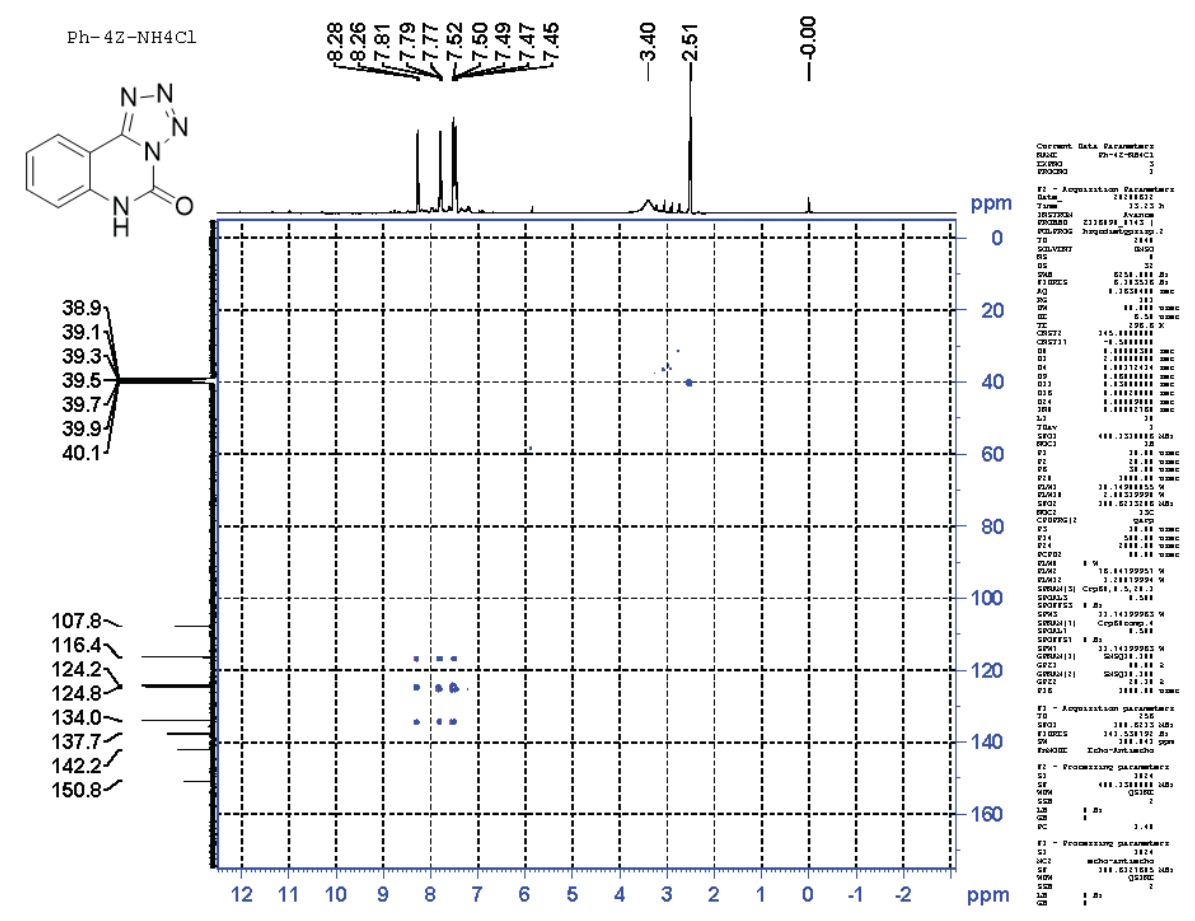

Figure S18. ${ }^{1} \mathrm{H}-{ }^{13} \mathrm{C}$ HSQC of $\mathbf{2 a - 2}$ in DMSO-d $\mathrm{d}_{6}$ operating at $100.61 \mathrm{MHz}$ (F1) / $400.13 \mathrm{MHz}$ (F2). 


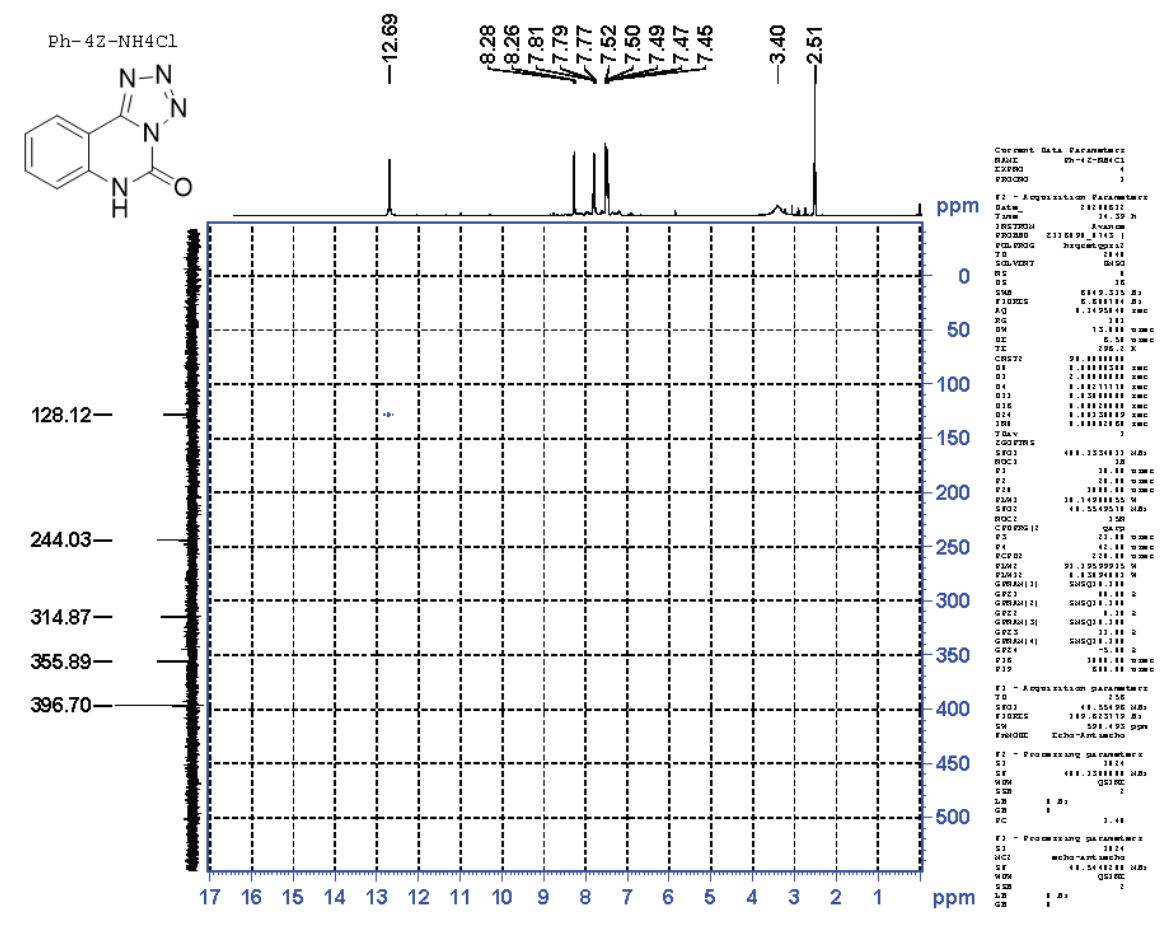

Figure S19. ${ }^{1} \mathrm{H}^{-15} \mathrm{~N}$ HSQC of $\mathbf{2 a - 2}$ in DMSO-d 6 operating at $40.54 \mathrm{MHz}$ (F1) / $400.13 \mathrm{MHz}$ (F2). 


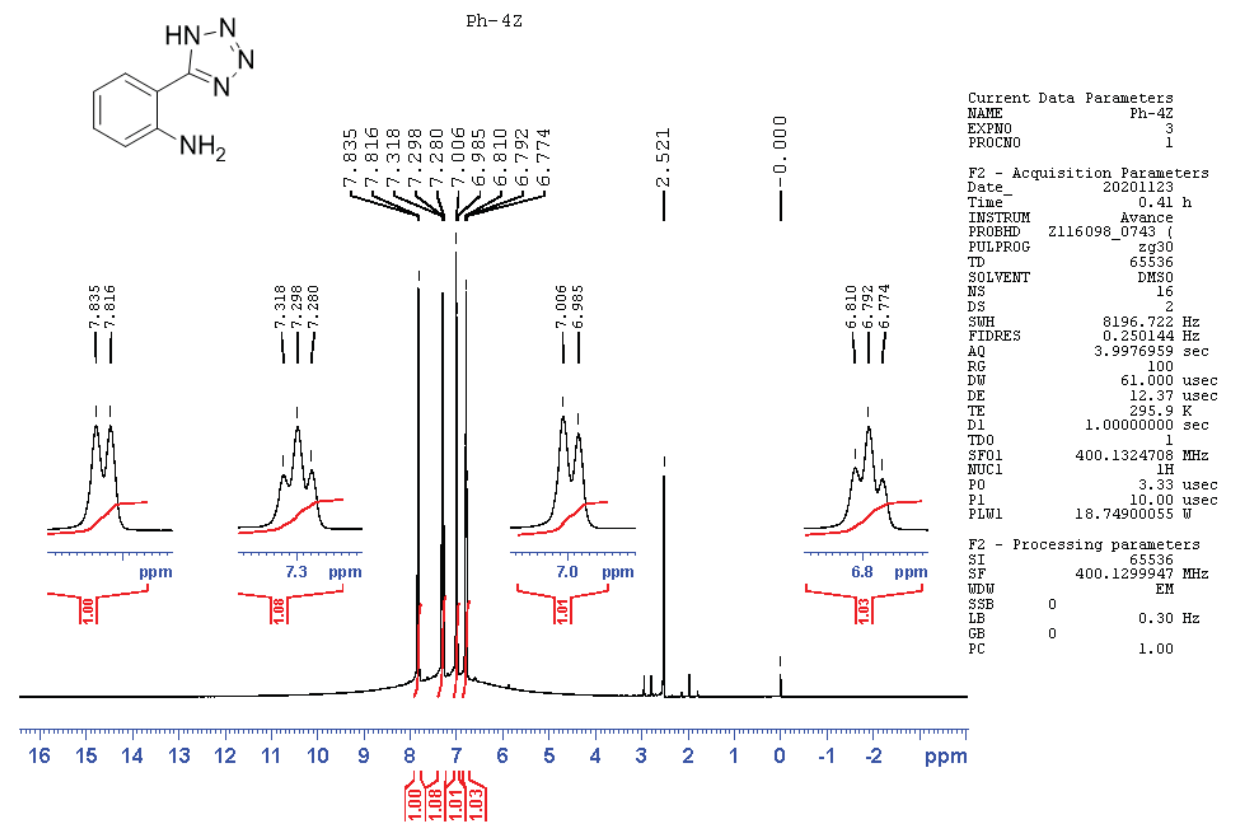

Figure $\mathrm{S} 20 .{ }^{1} \mathrm{H}$ NMR of $\mathbf{2 a}$ in DMSO- $\mathrm{d}_{6}$ operating at $400.13 \mathrm{MHz}$.

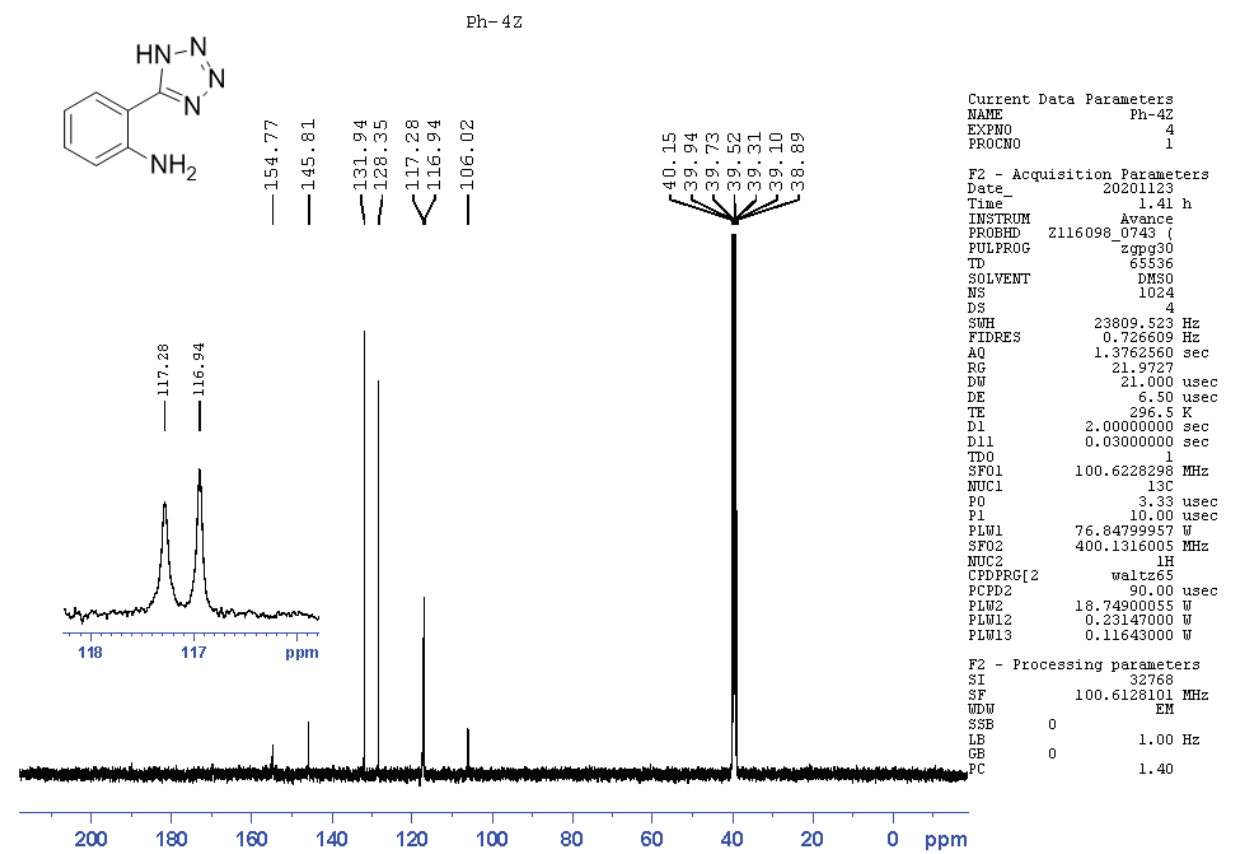

Figure $\mathrm{S} 21 .{ }^{13} \mathrm{C}$ NMR of $2 \mathrm{a}$ in DMSO- $\mathrm{d}_{6}$ operating at $100.61 \mathrm{MHz}$. 


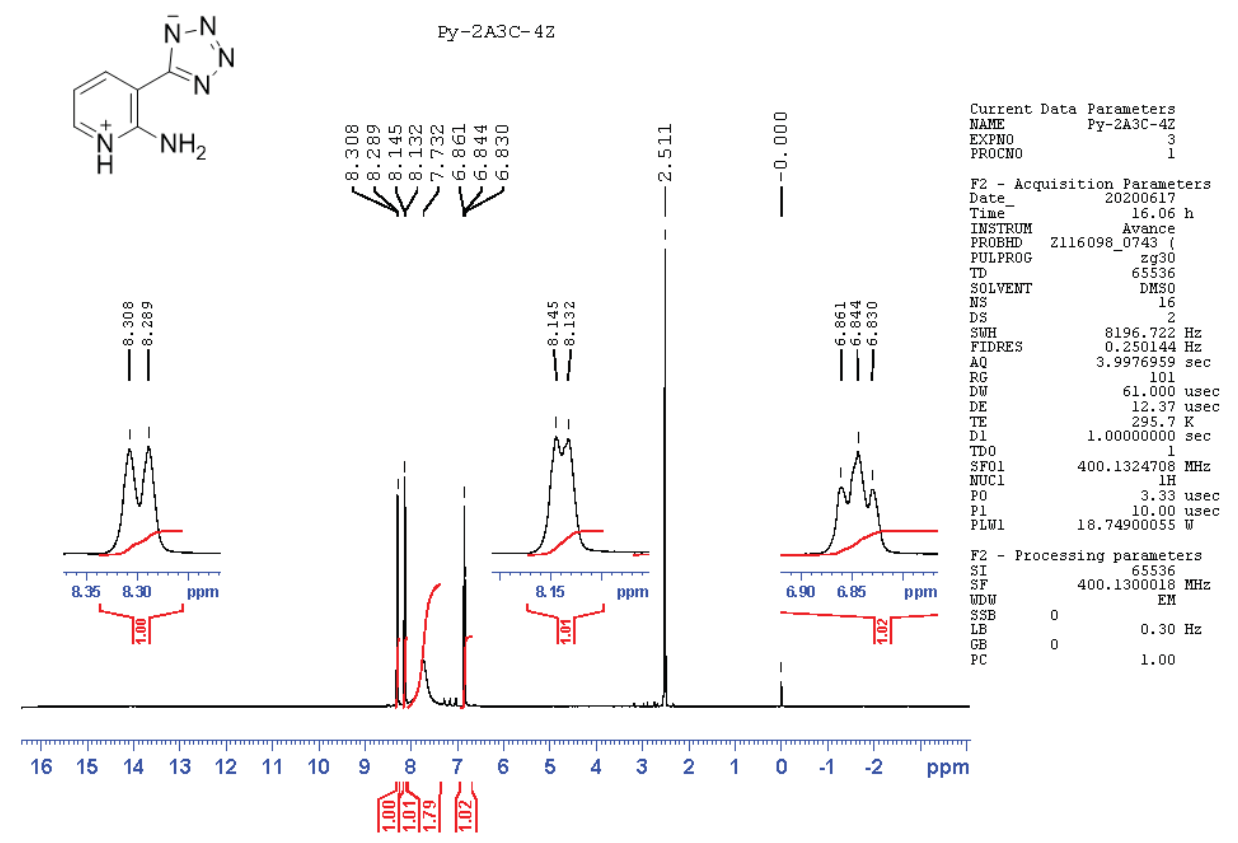

Figure $\mathrm{S} 22 .{ }^{1} \mathrm{H}$ NMR of $\mathbf{2} \mathbf{b}$ in DMSO-d $\mathrm{d}_{6}$ operating at $400.13 \mathrm{MHz}$.

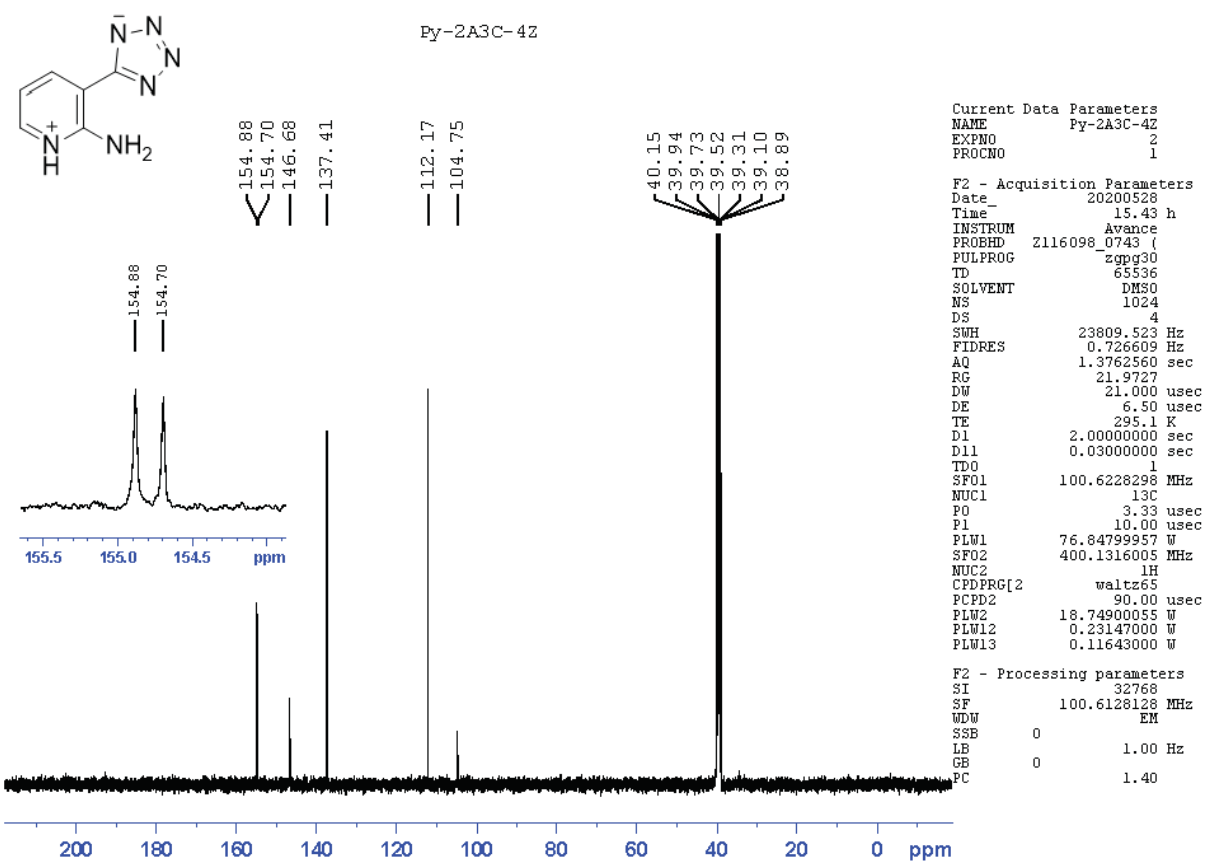

Figure $\mathrm{S} 23 .{ }^{13} \mathrm{C}$ NMR of $\mathbf{2} \mathbf{b}$ in DMSO- $\mathrm{d}_{6}$ operating at $100.61 \mathrm{MHz}$. 


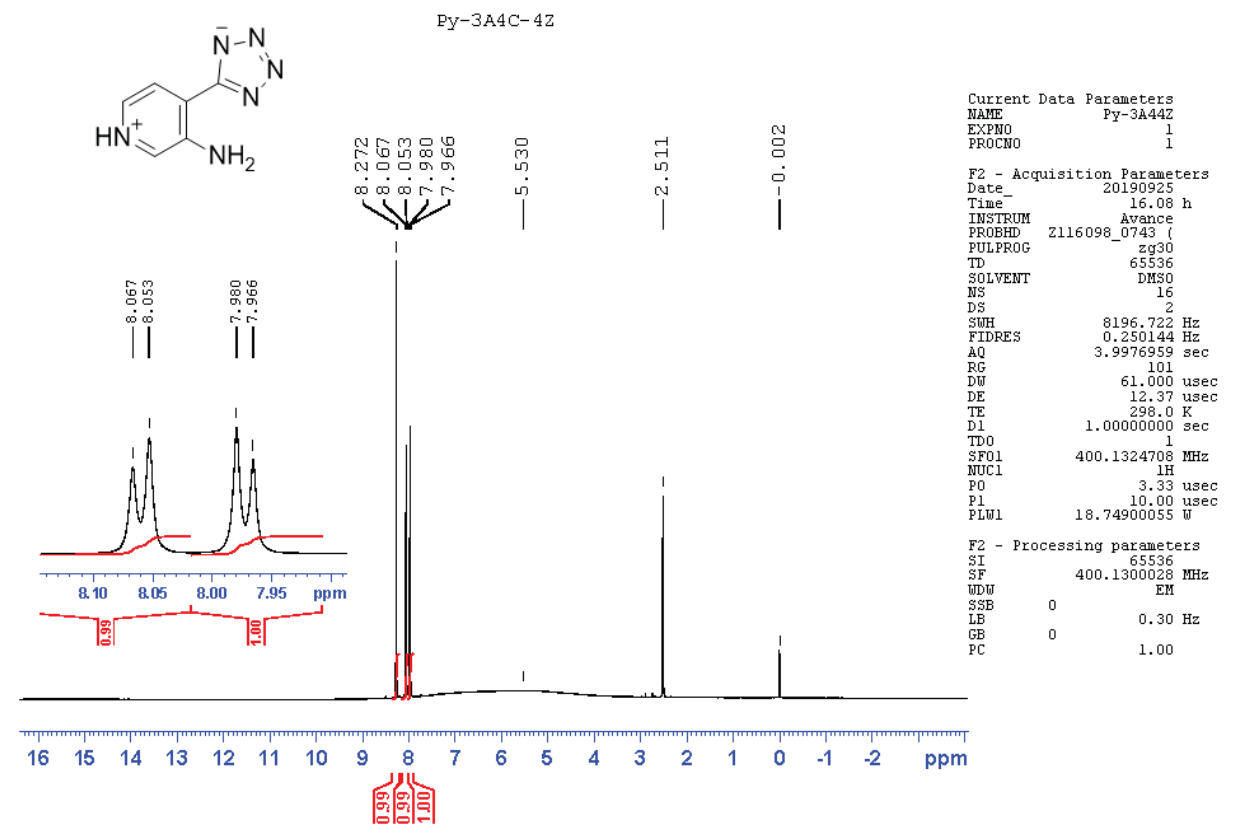

Figure $\mathrm{S} 24 .{ }^{1} \mathrm{H}$ NMR of $2 \mathrm{c}$ in DMSO- $\mathrm{d}_{6}$ operating at $400.13 \mathrm{MHz}$.

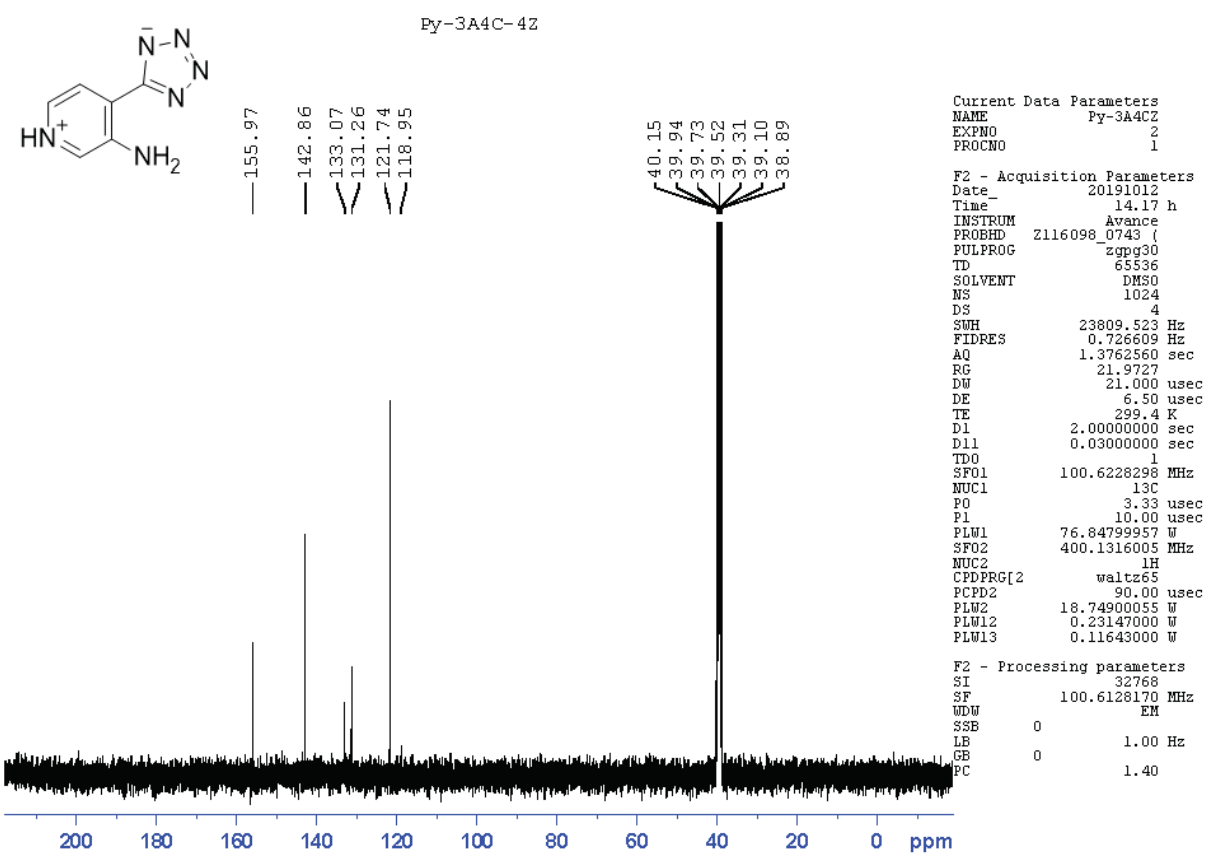

Figure $\mathrm{S} 25 .{ }^{13} \mathrm{C}$ NMR of $\mathbf{2 c}$ in DMSO- $\mathrm{d}_{6}$ operating at $100.61 \mathrm{MHz}$. 


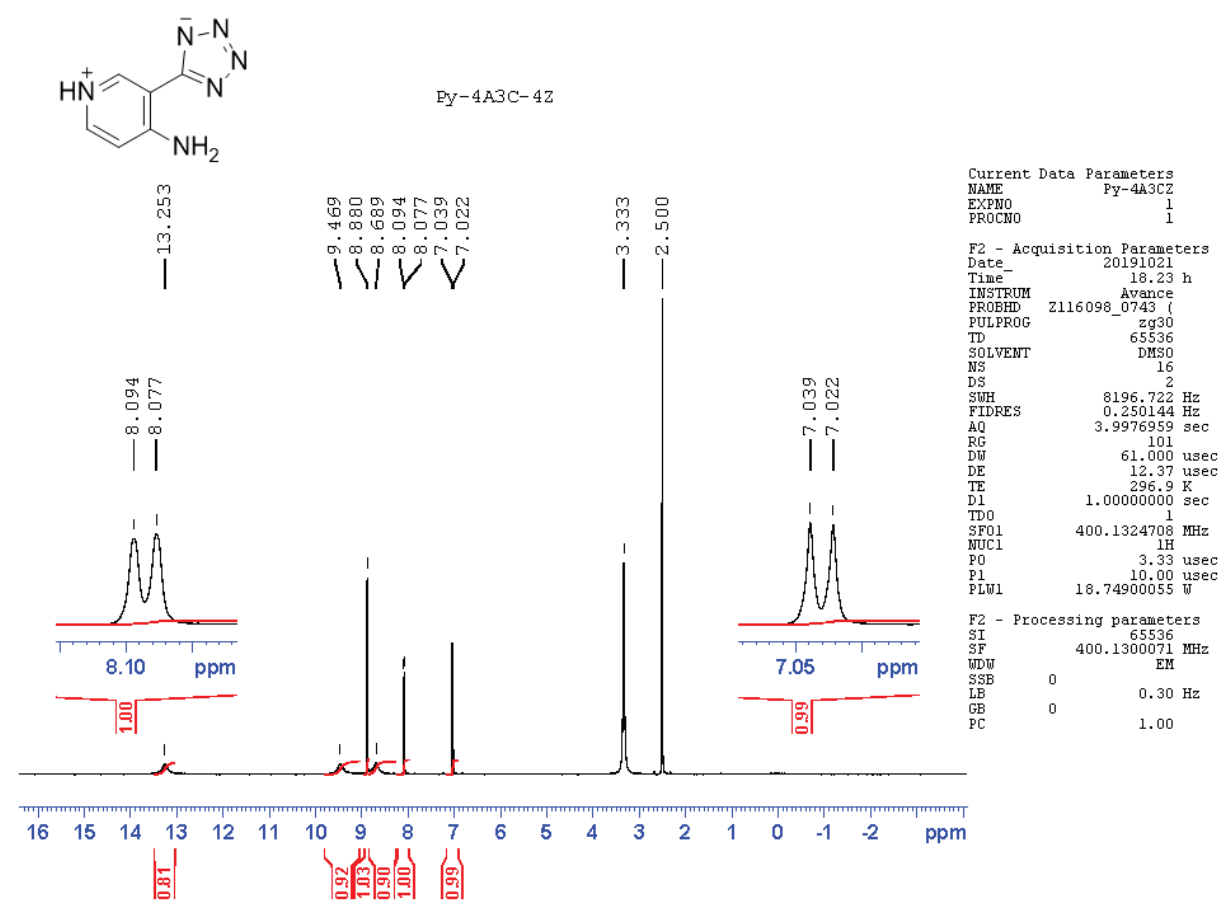

Figure $\mathrm{S} 26 .{ }^{1} \mathrm{H}$ NMR of $\mathbf{2} \mathbf{d}$ in DMSO-d $\mathrm{d}_{6}$ operating at $400.13 \mathrm{MHz}$.

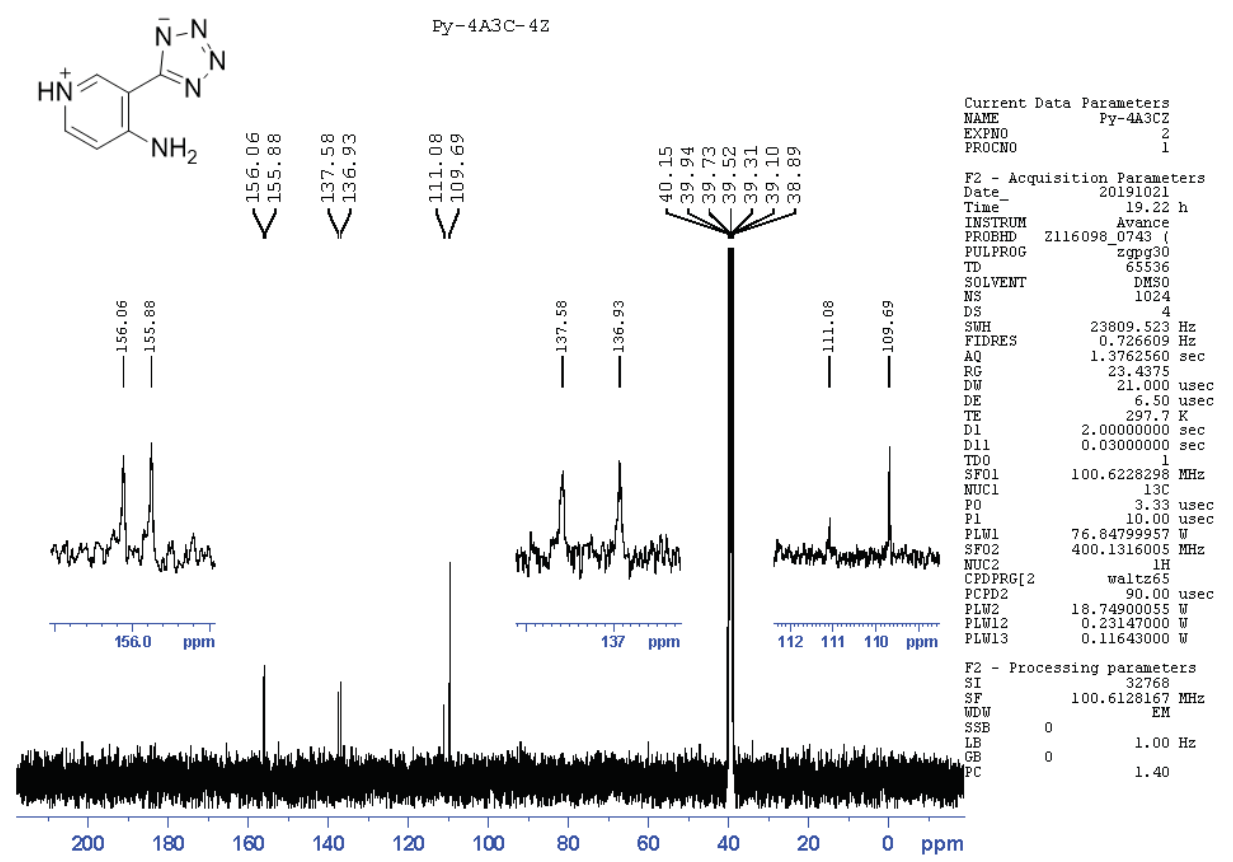

Figure S27. ${ }^{13} \mathrm{C}$ NMR of $\mathbf{2} \mathbf{d}$ in DMSO- $\mathrm{d}_{6}$ operating at $100.61 \mathrm{MHz}$. 


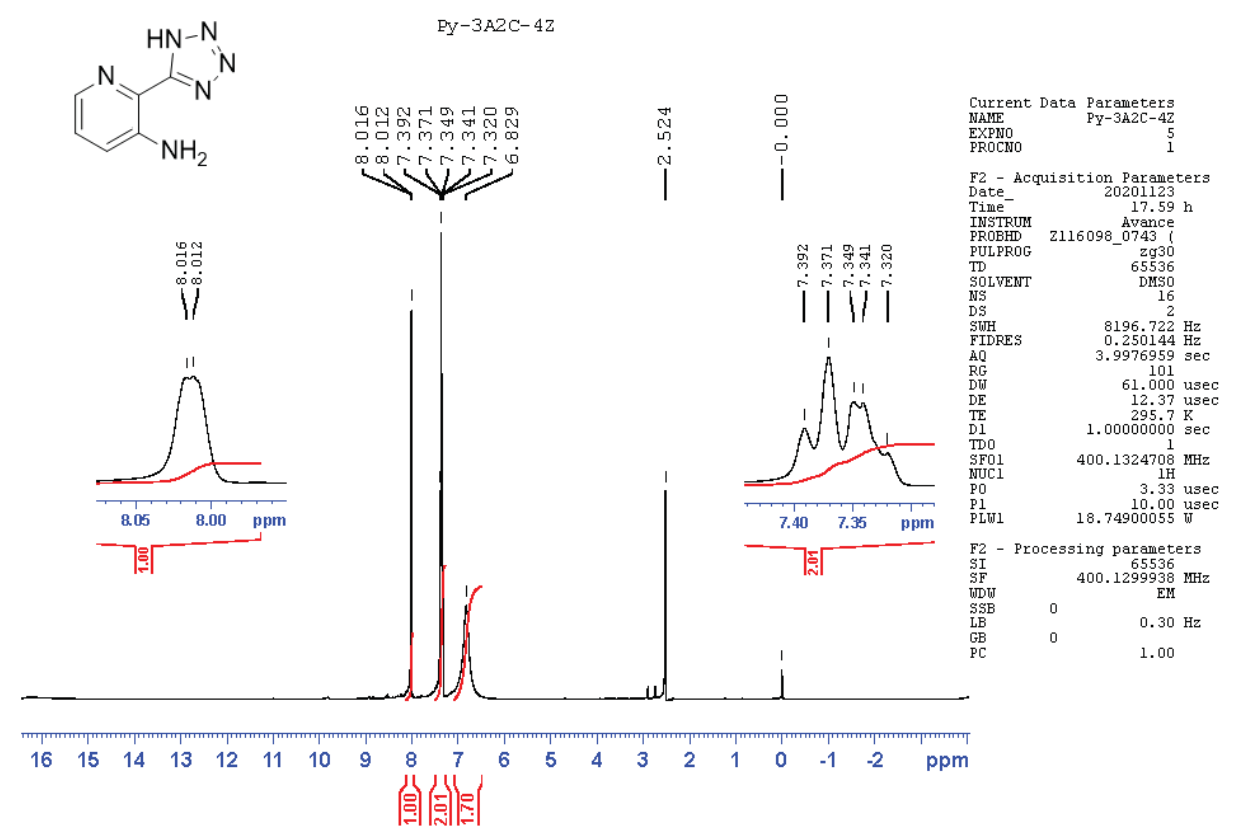

Figure S28. ${ }^{1} \mathrm{H}$ NMR of $2 \mathrm{e}$ in DMSO- $\mathrm{d}_{6}$ operating at $400.13 \mathrm{MHz}$.

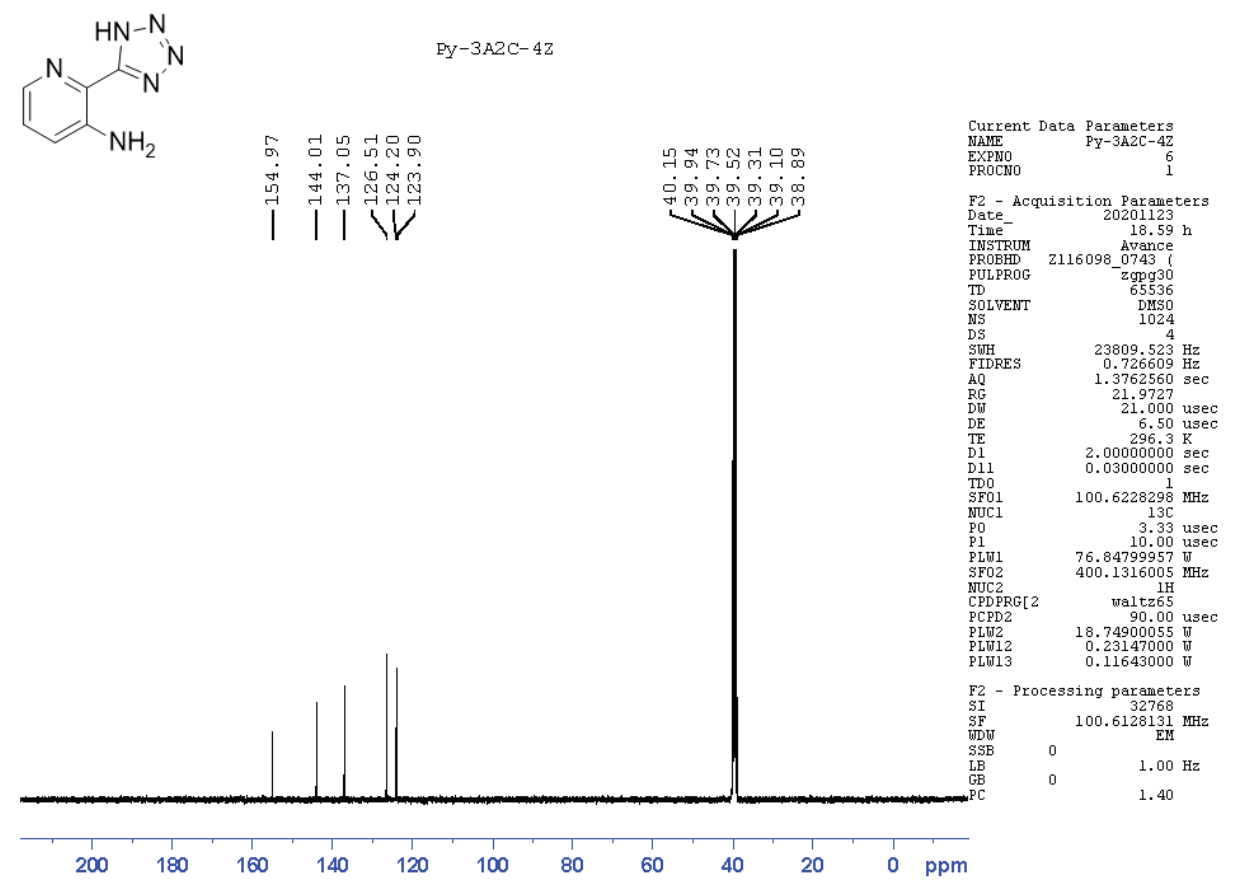

Figure S29. ${ }^{13} \mathrm{C}$ NMR of $2 \mathrm{e}$ in DMSO-d $\mathrm{d}_{6}$ operating at $100.61 \mathrm{MHz}$. 


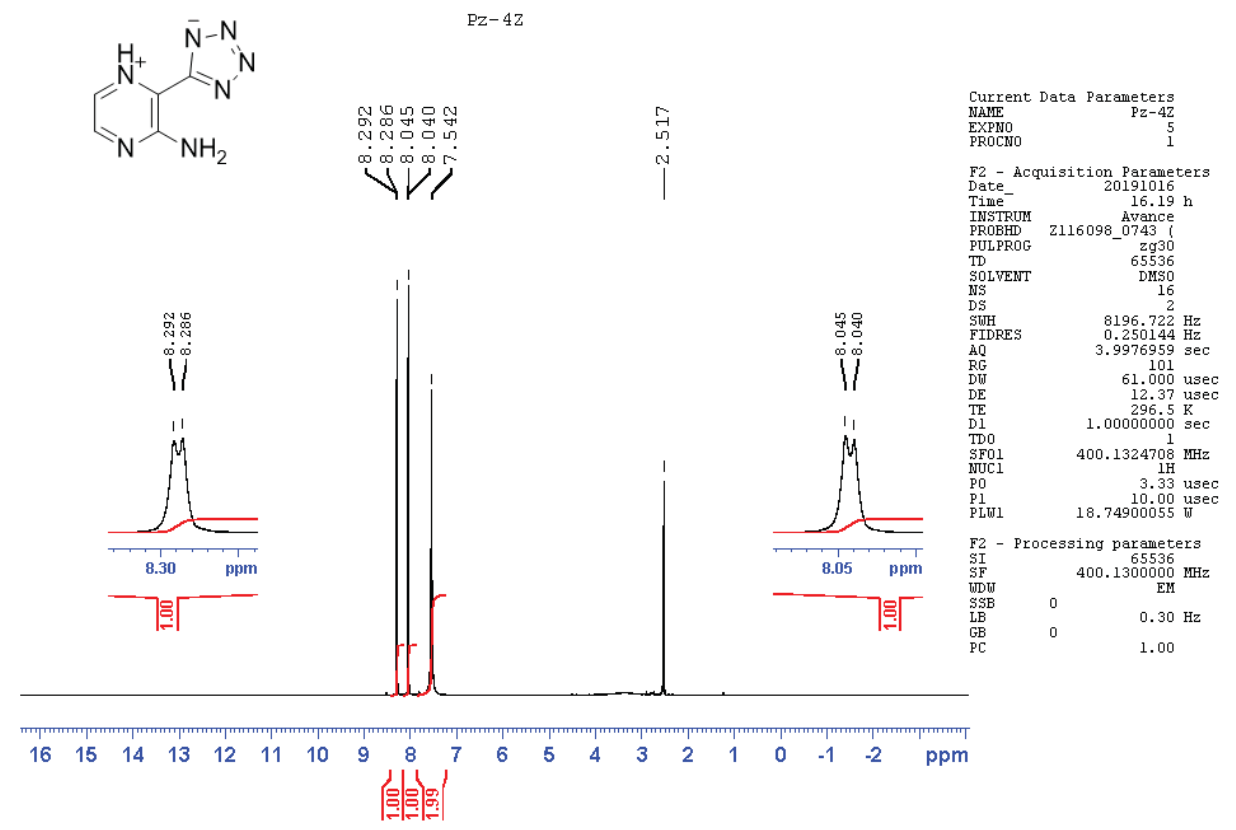

Figure $\mathrm{S} 30 .{ }^{1} \mathrm{H}$ NMR of $\mathbf{2} f$ in DMSO- $\mathrm{d}_{6}$ operating at $400.13 \mathrm{MHz}$.

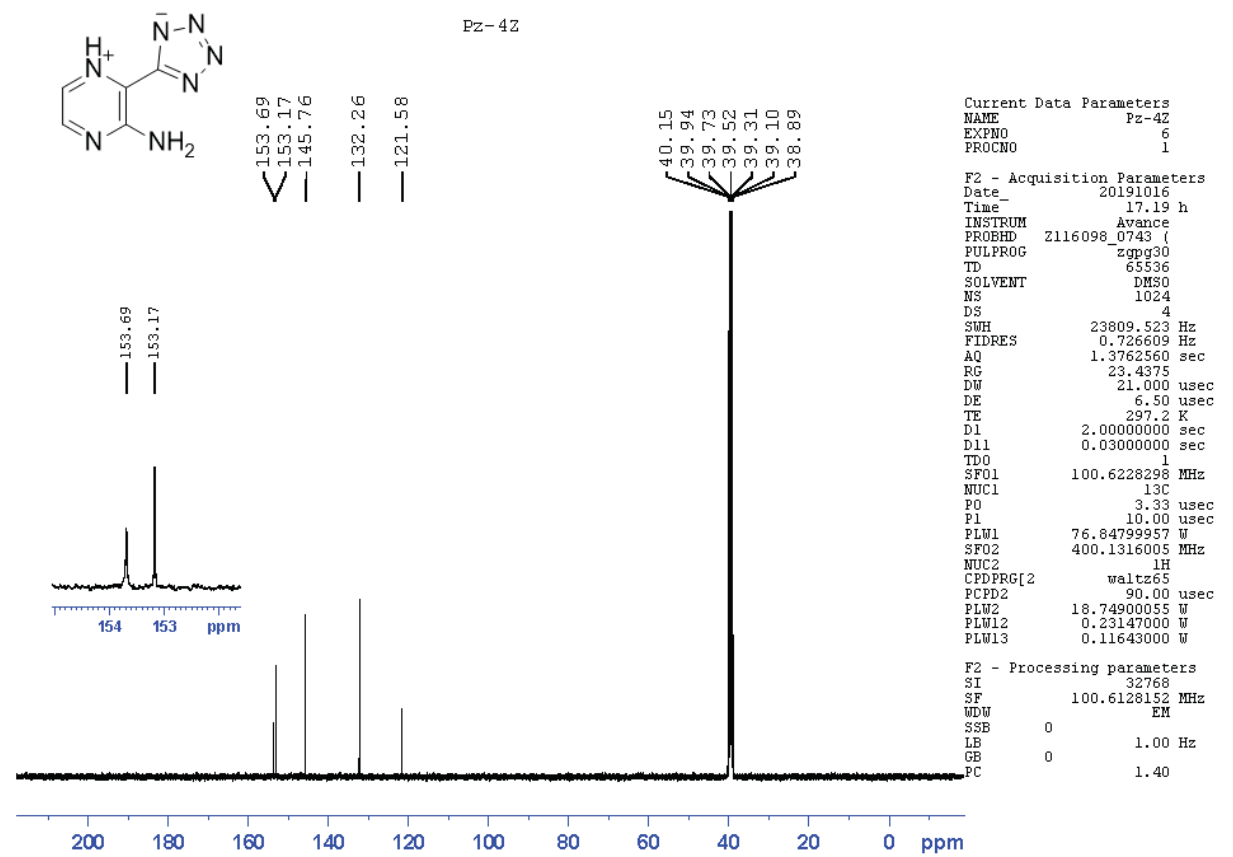

Figure S31. ${ }^{13} \mathrm{C}$ NMR of $\mathbf{2} f$ in DMSO- $\mathrm{d}_{6}$ operating at $100.61 \mathrm{MHz}$. 


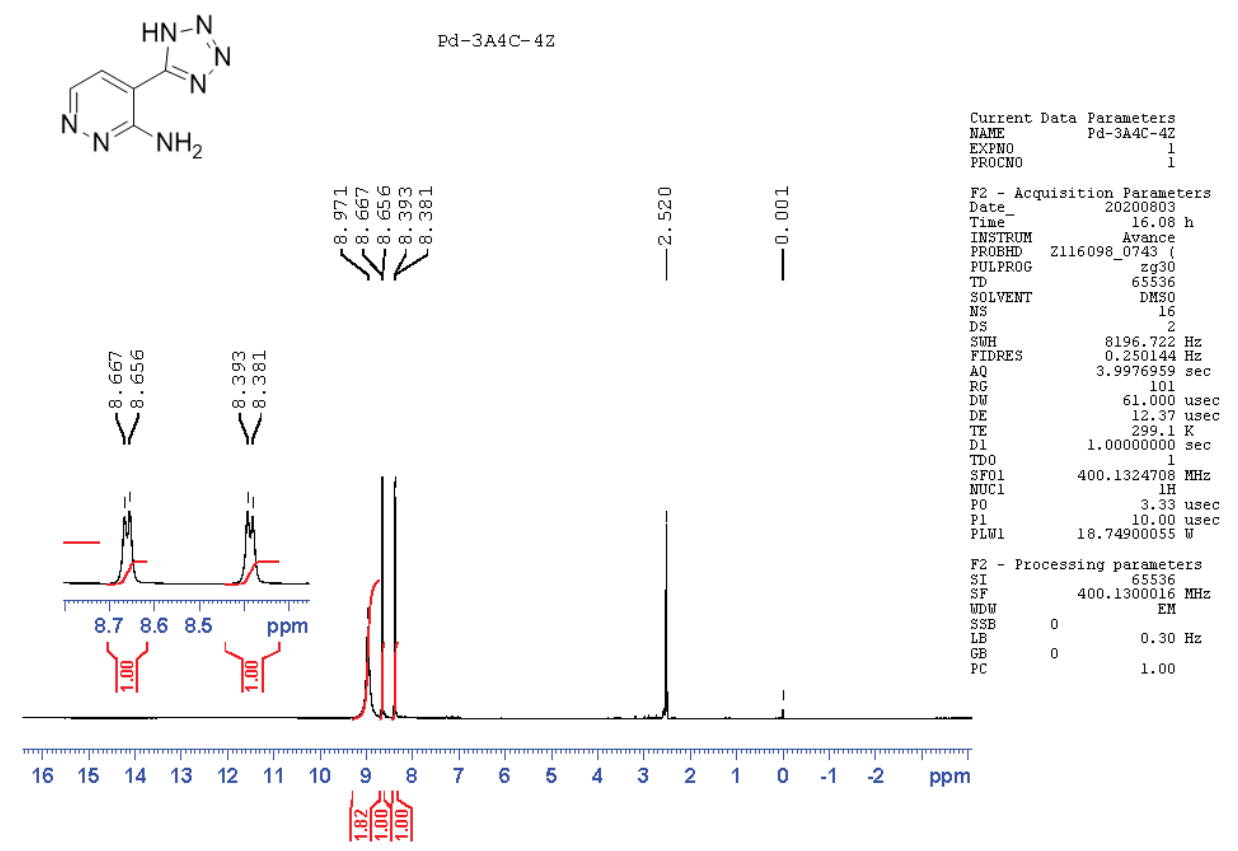

Figure S32. ${ }^{1} \mathrm{H}$ NMR of $\mathbf{2 g}$ in DMSO-d 6 operating at $400.13 \mathrm{MHz}$.

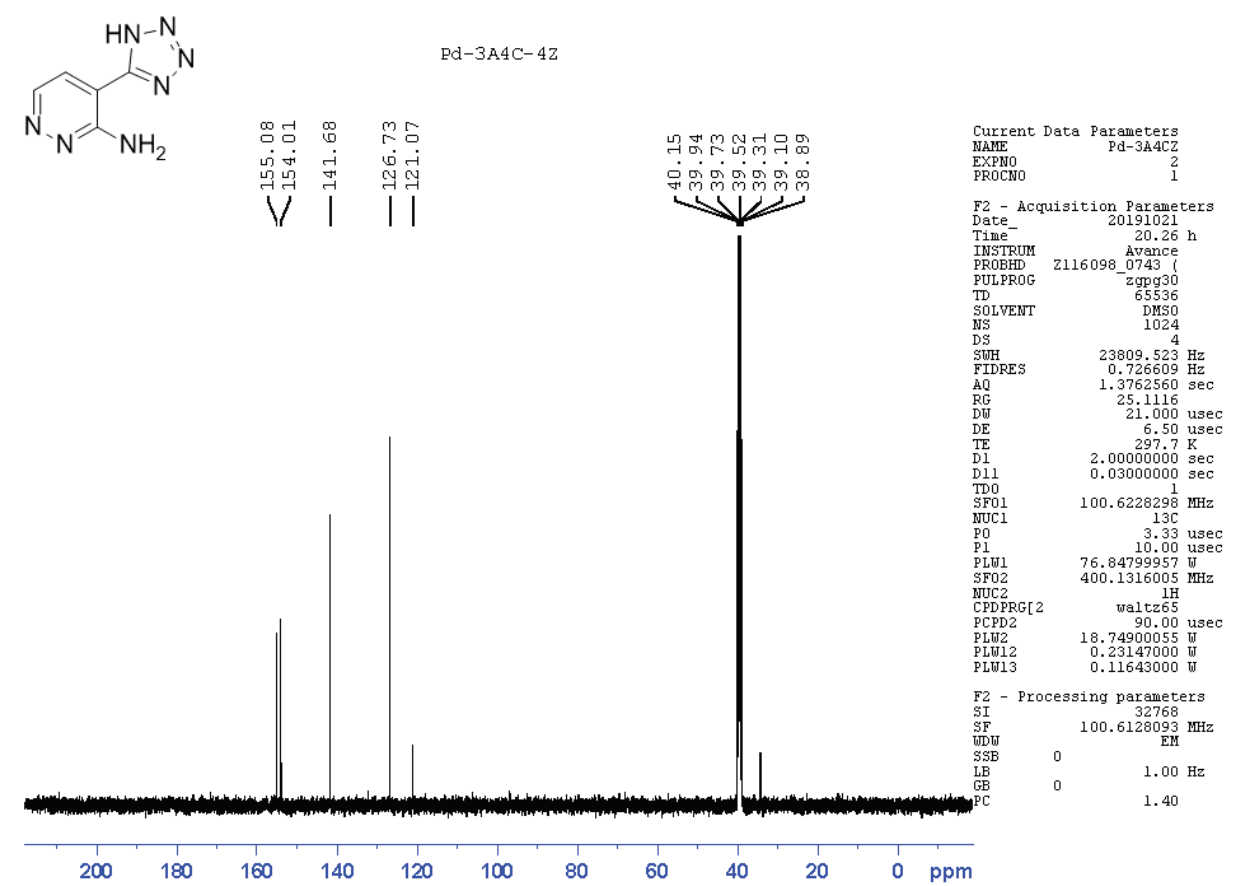

Figure S33. ${ }^{13} \mathrm{C}$ NMR of $\mathbf{2 g}$ in DMSO-d $\mathrm{d}_{6}$ operating at $100.61 \mathrm{MHz}$. 


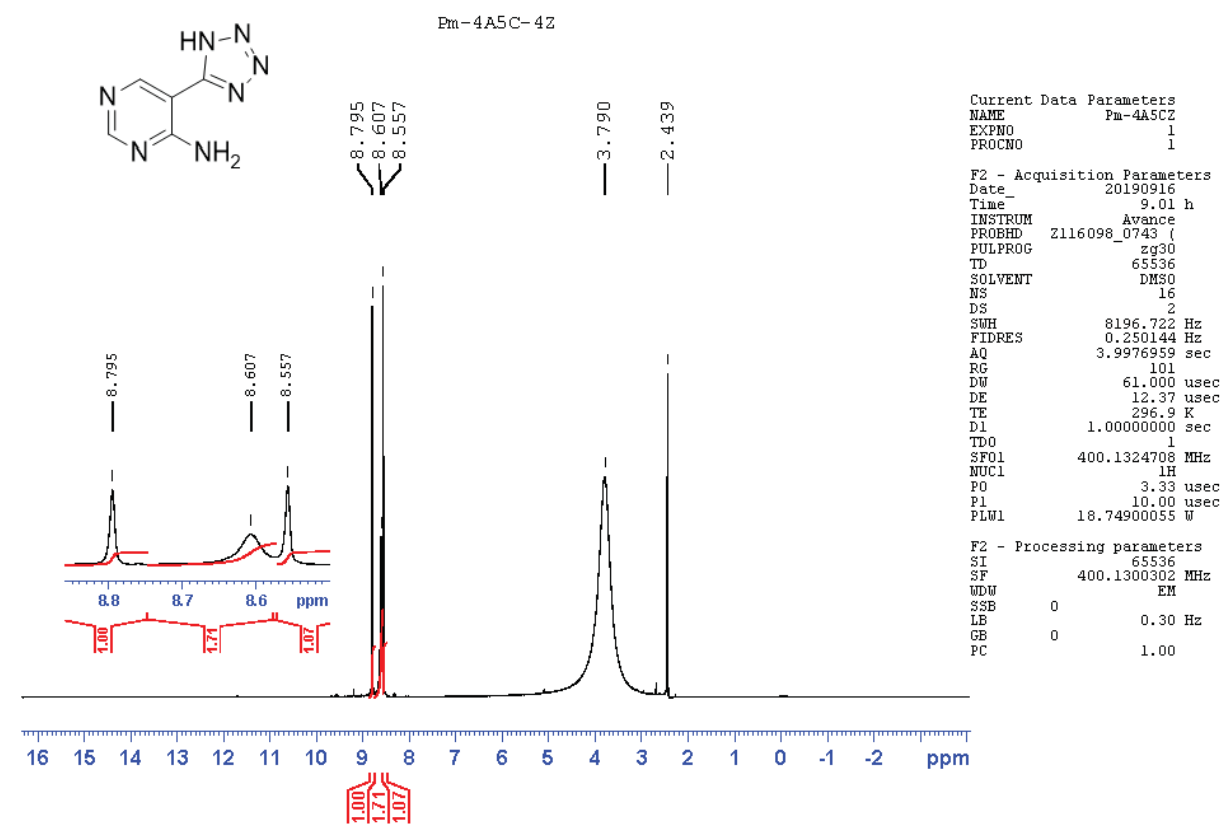

Figure S34. ${ }^{1} \mathrm{H}$ NMR of $\mathbf{2} \mathbf{h}$ in DMSO- $\mathrm{d}_{6}$ operating at $400.13 \mathrm{MHz}$.

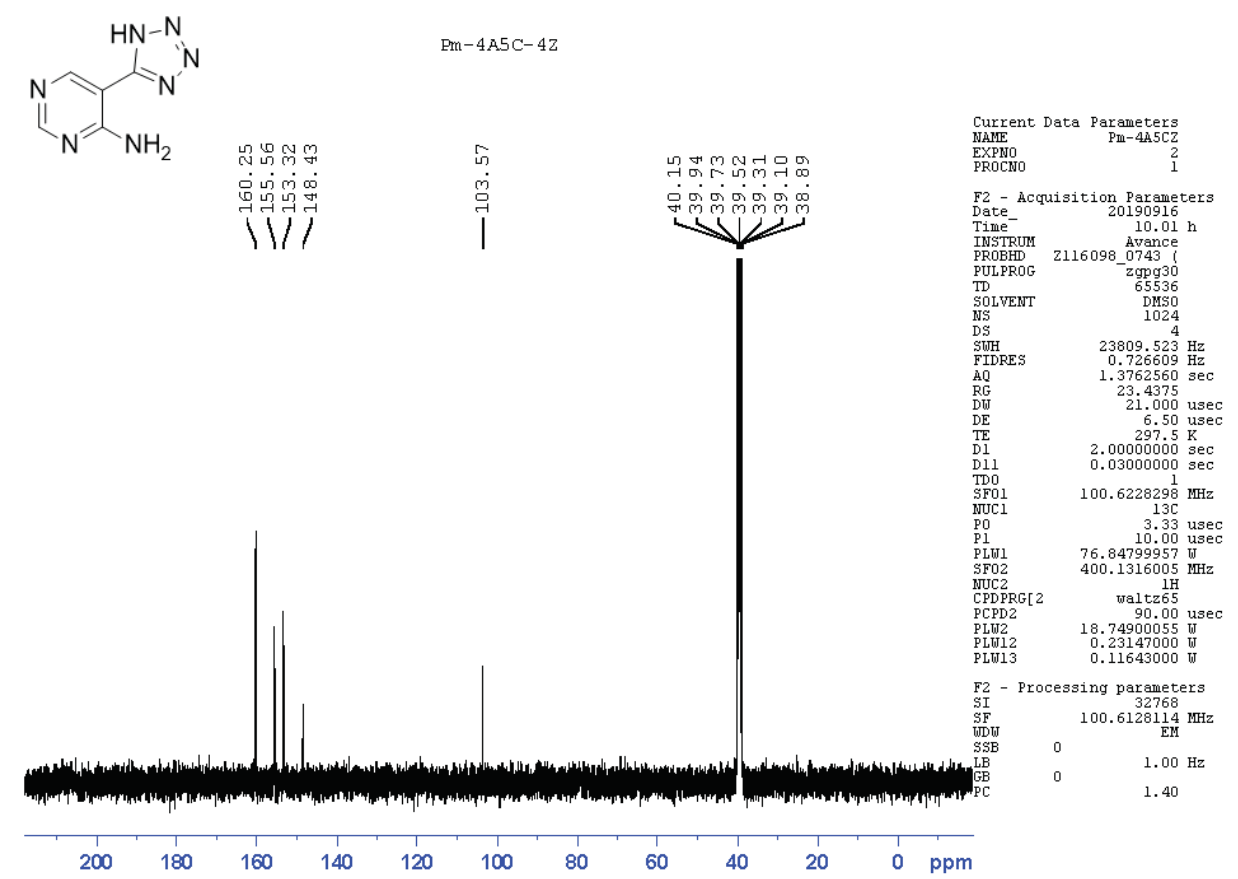

Figure S35. ${ }^{13} \mathrm{C}$ NMR of $\mathbf{2} \mathbf{h}$ in DMSO- $\mathrm{d}_{6}$ operating at $100.61 \mathrm{MHz}$. 


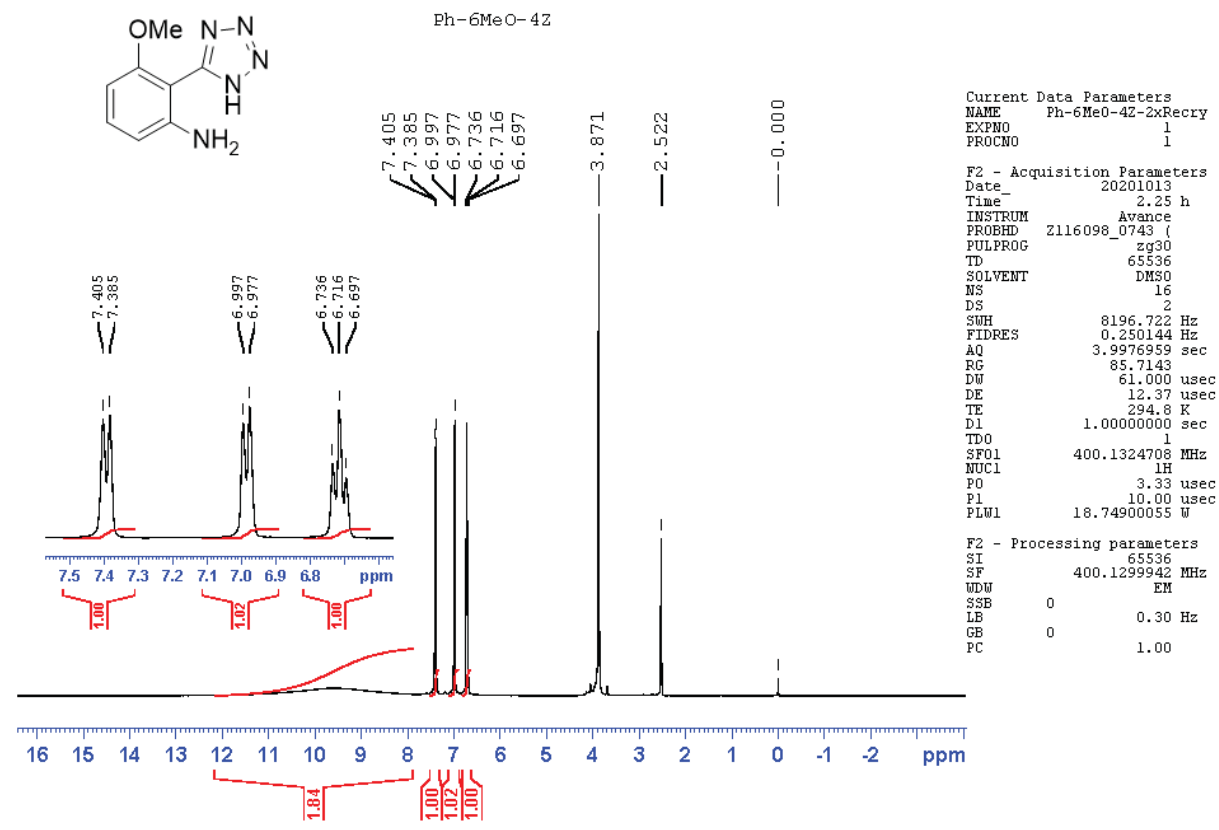

Figure S36. ${ }^{1} \mathrm{H}$ NMR of $\mathbf{2 k}$ in DMSO-d 6 operating at $400.13 \mathrm{MHz}$.

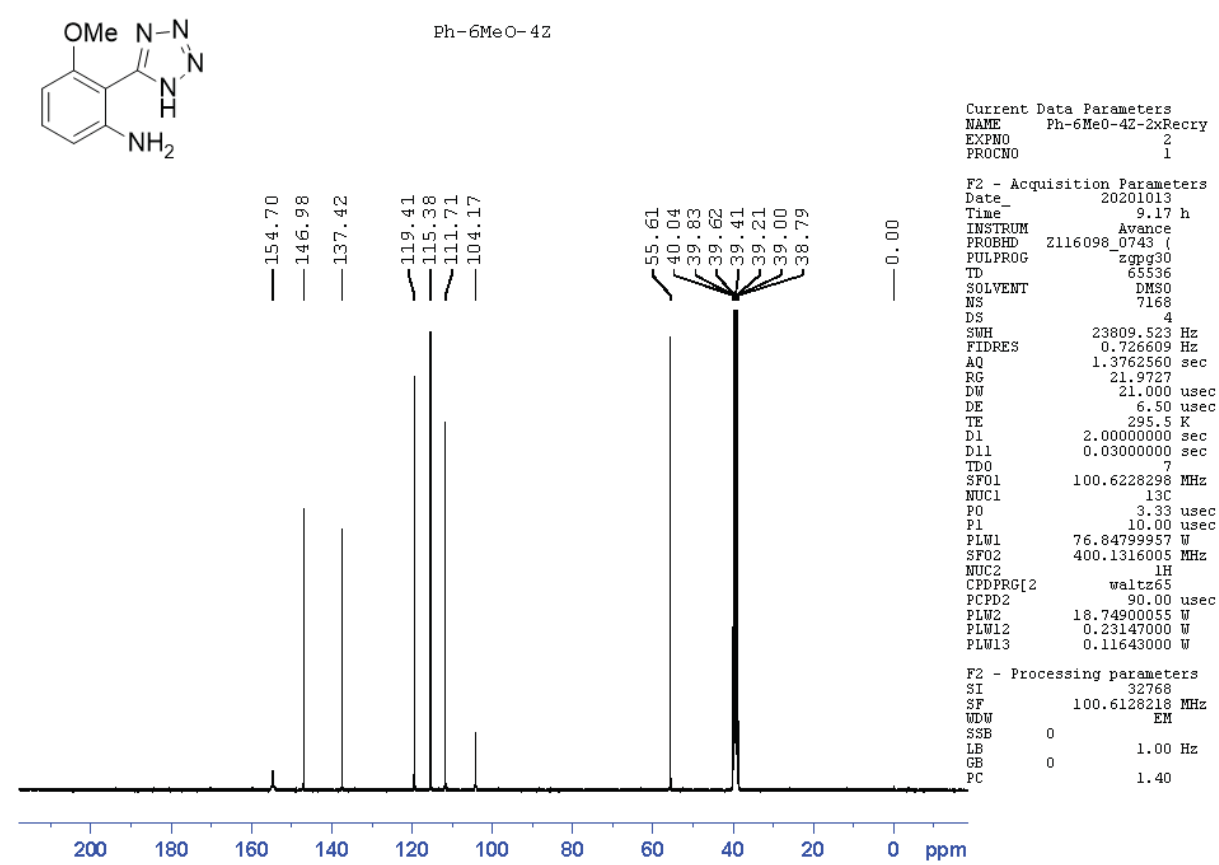

Figure S37. ${ }^{13} \mathrm{C}$ NMR of $\mathbf{2 k}$ in DMSO- $\mathrm{d}_{6}$ operating at $100.61 \mathrm{MHz}$. 


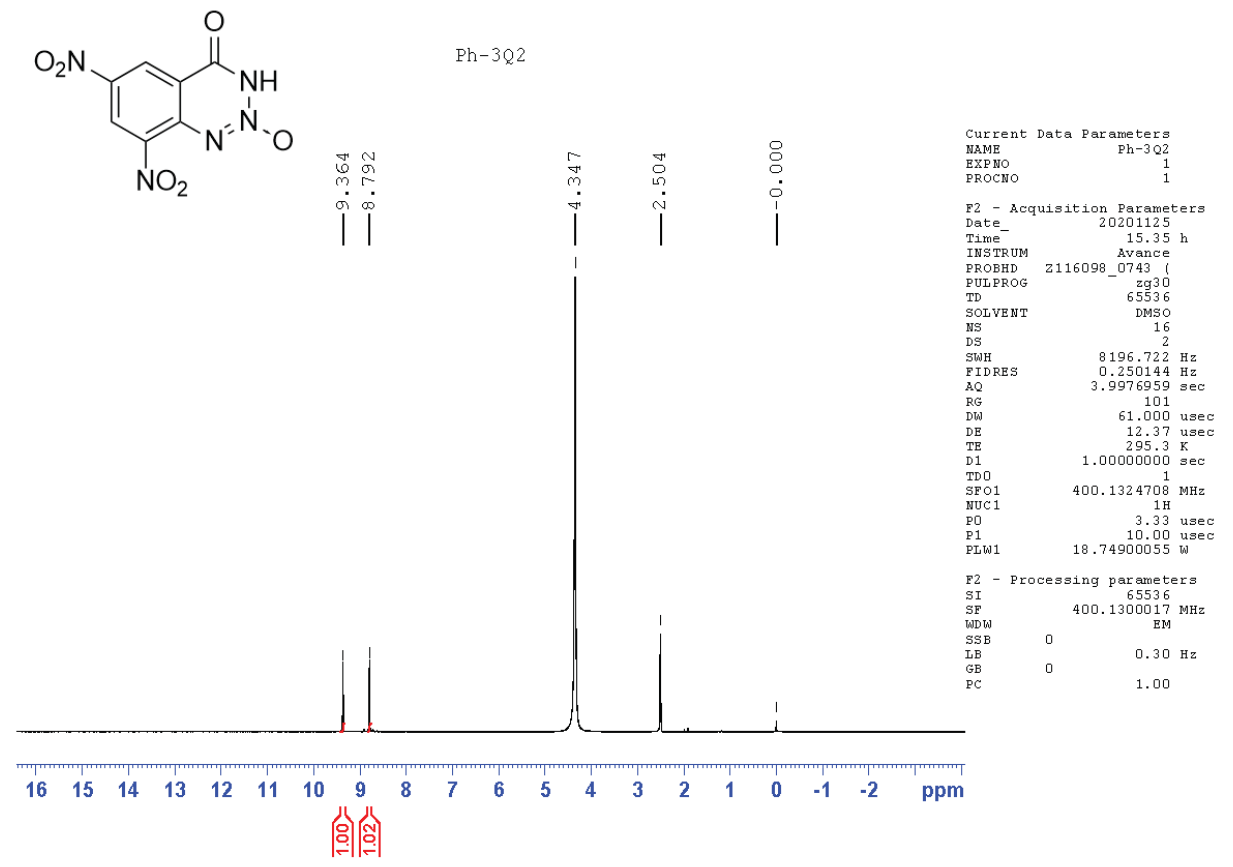

Figure S38. ${ }^{1} \mathrm{H}$ NMR of 3a-1 in DMSO- $\mathrm{d}_{6}$ operating at $400.13 \mathrm{MHz}$.

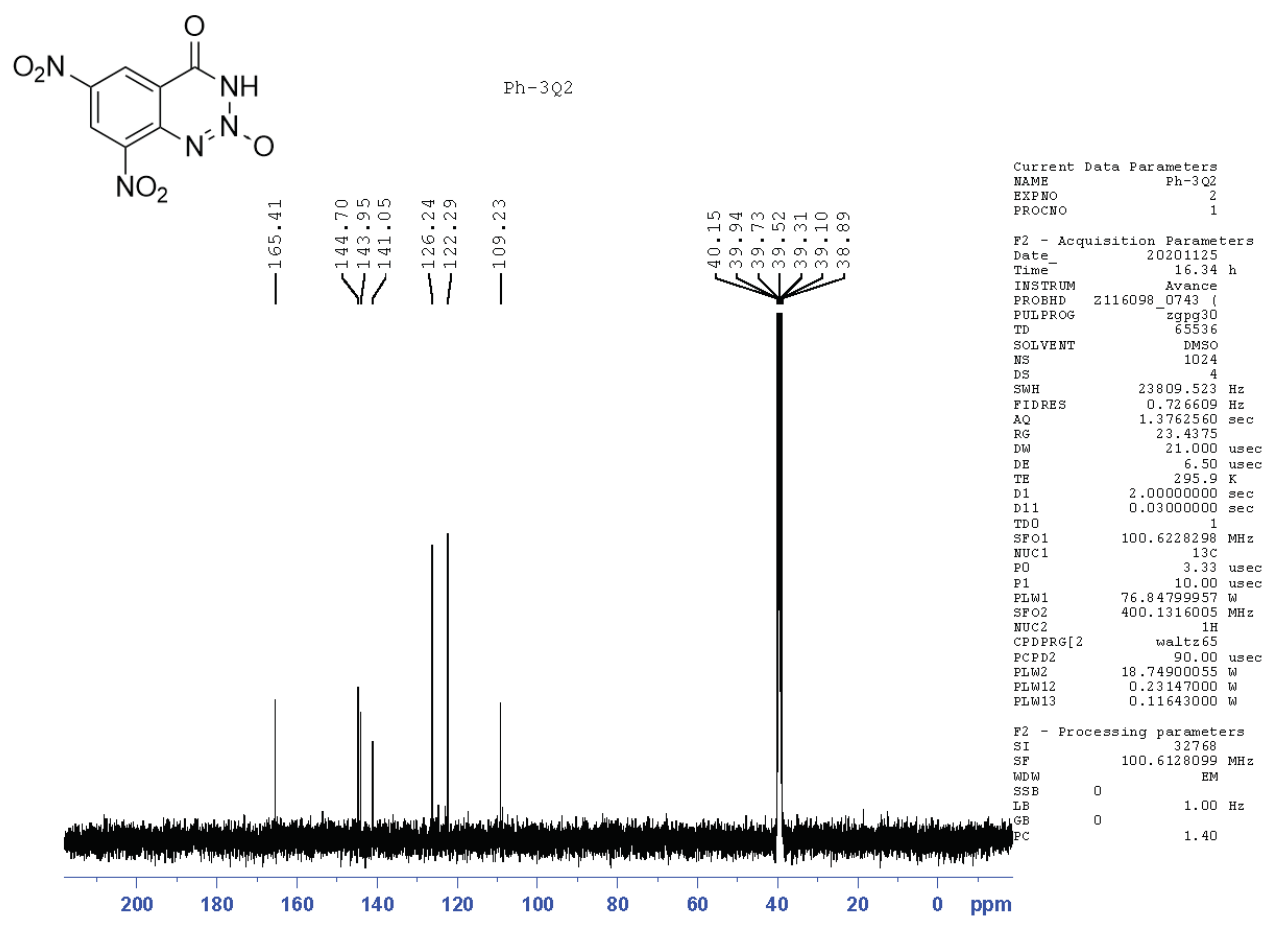

Figure S39. ${ }^{13} \mathrm{C}$ NMR of $\mathbf{3 a - 1}$ in DMSO-d $\mathrm{d}_{6}$ operating at $100.61 \mathrm{MHz}$. 


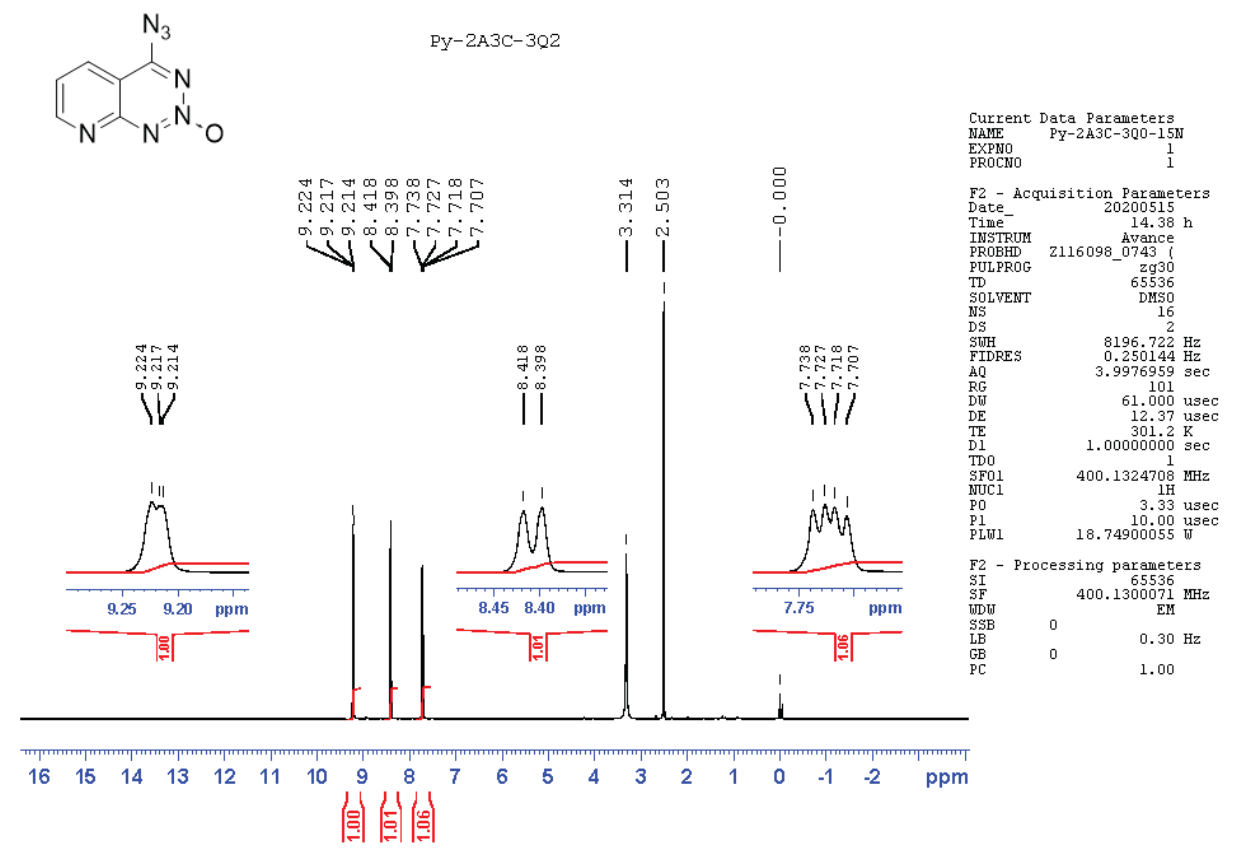

Figure $\mathrm{S} 40 .{ }^{1} \mathrm{H}$ NMR of $\mathbf{3 b}$ in DMSO-d $\mathrm{d}_{6}$ operating at $400.13 \mathrm{MHz}$.

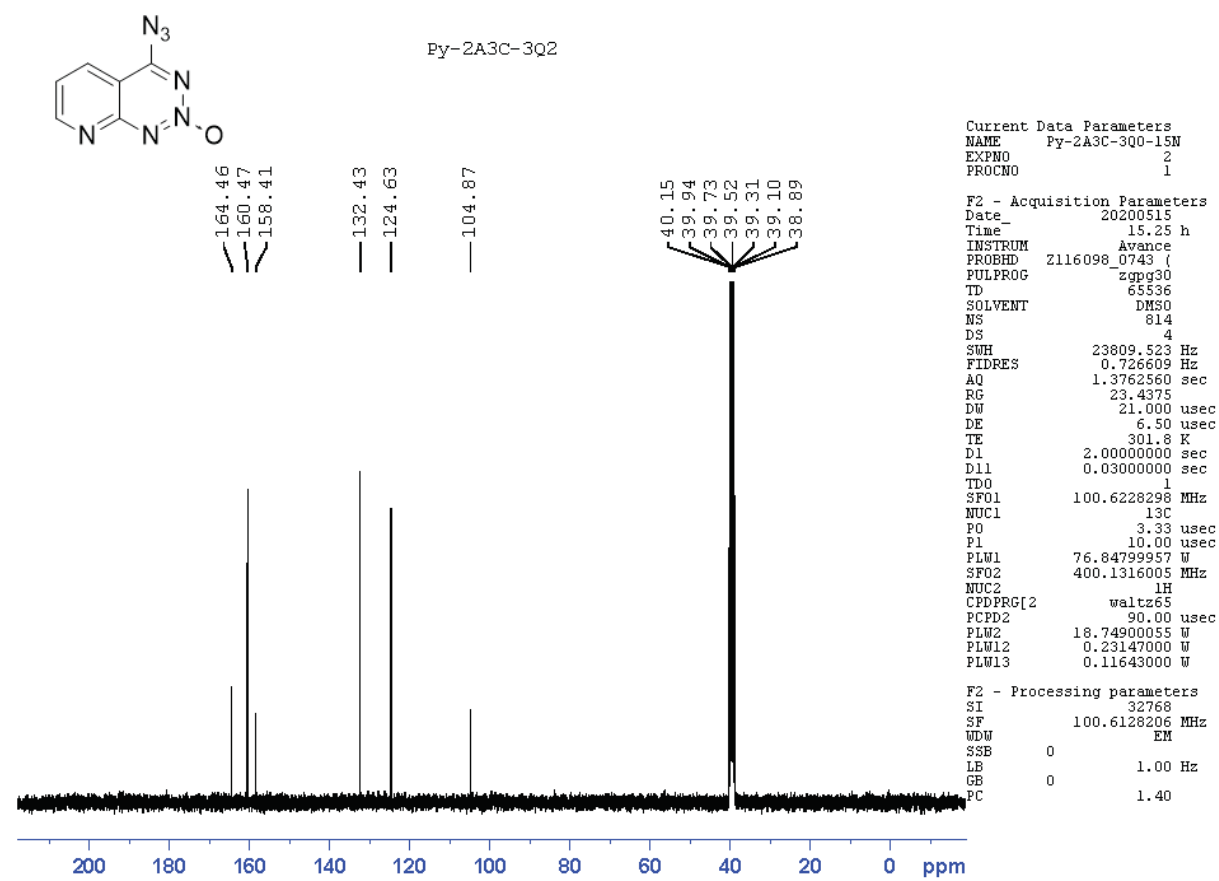

Figure $\mathrm{S} 41 .{ }^{13} \mathrm{C}$ NMR of $\mathbf{3 b}$ in DMSO- $\mathrm{d}_{6}$ operating at $100.61 \mathrm{MHz}$. 


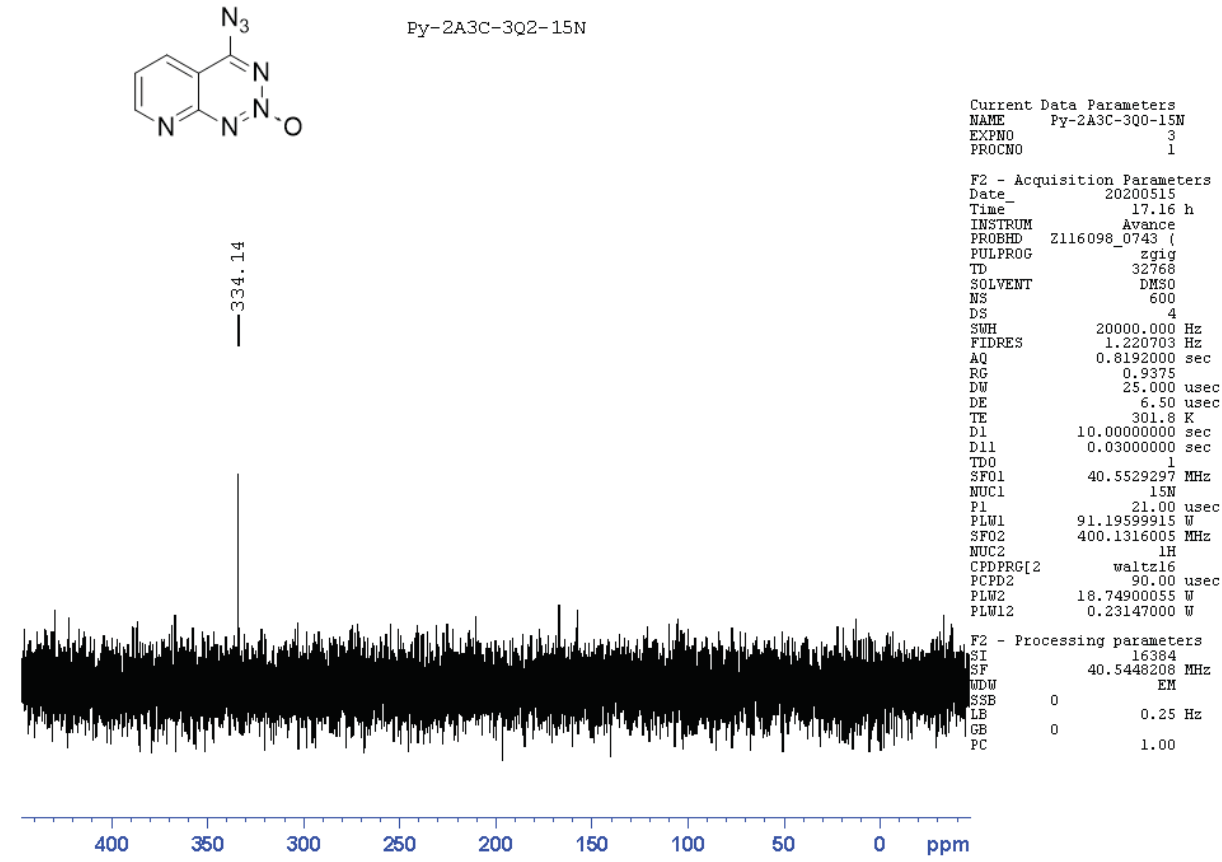

Figure S42. ${ }^{15} \mathrm{~N}$ NMR of ${ }^{15} \mathrm{~N}$-labeled $\mathbf{3 b}$ in DMSO- $\mathrm{d}_{6}$ operating at $40.54 \mathrm{MHz}$. 


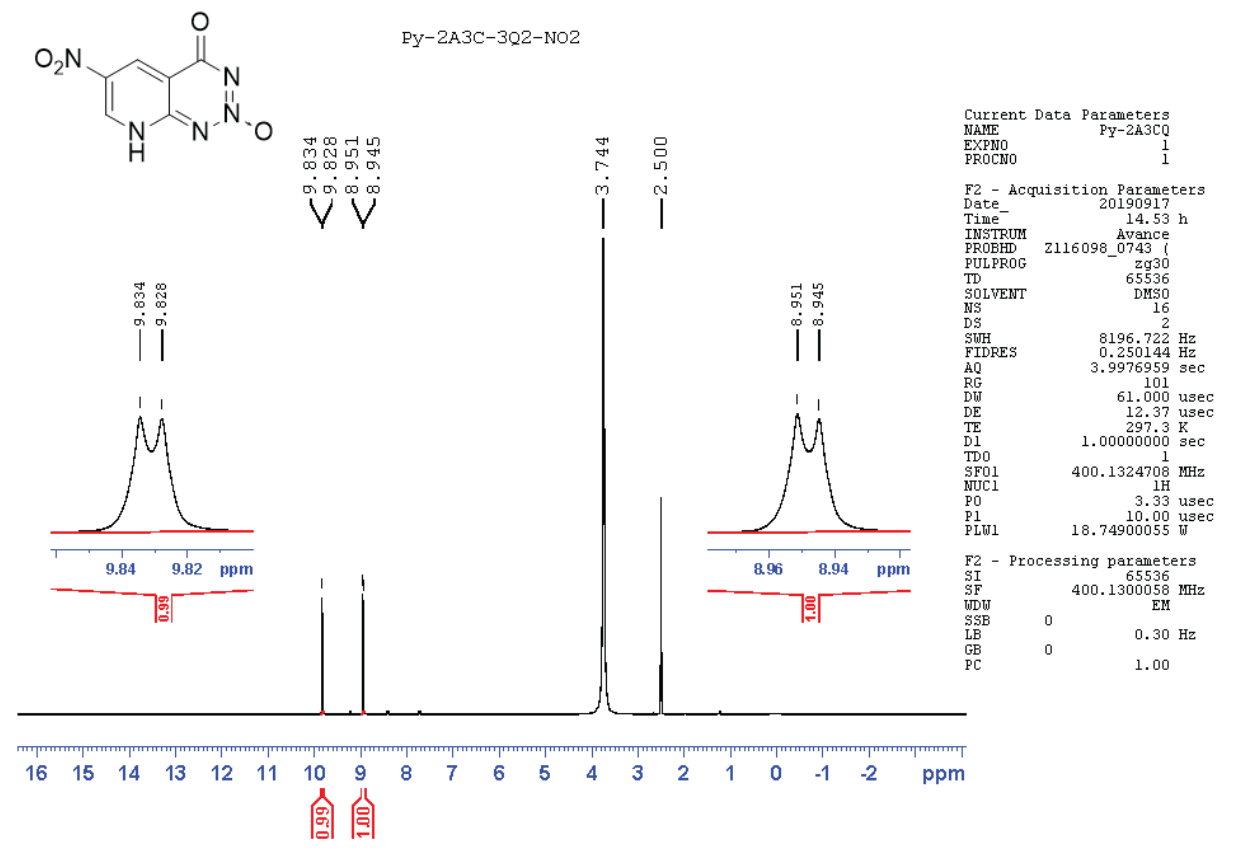

Figure S43. ${ }^{1} \mathrm{H}$ NMR of $\mathbf{3 b - 1}$ in DMSO-d $\mathrm{d}_{6}$ operating at $400.13 \mathrm{MHz}$.

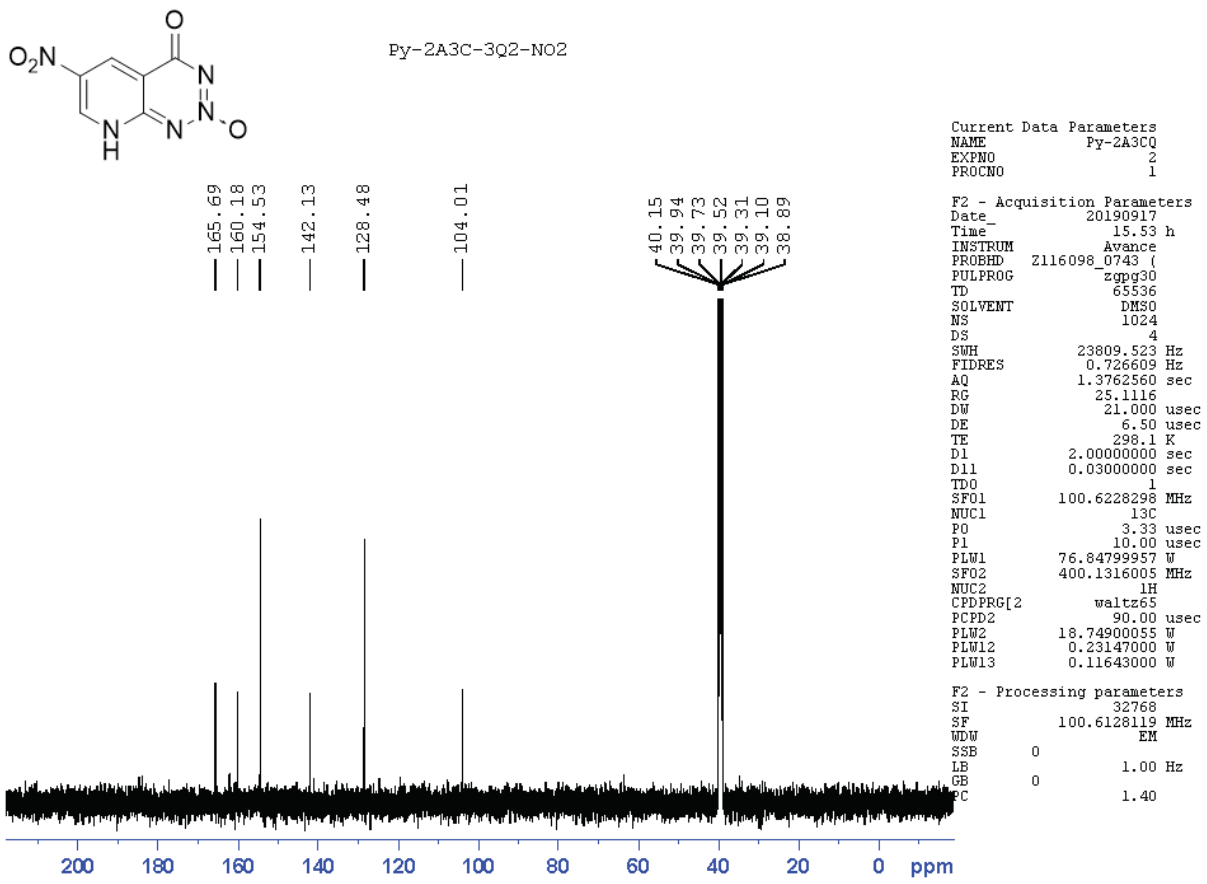

Figure S44. ${ }^{13} \mathrm{C}$ NMR of $\mathbf{3 b - 1}$ in DMSO-d $\mathrm{d}_{6}$ operating at $100.61 \mathrm{MHz}$. 


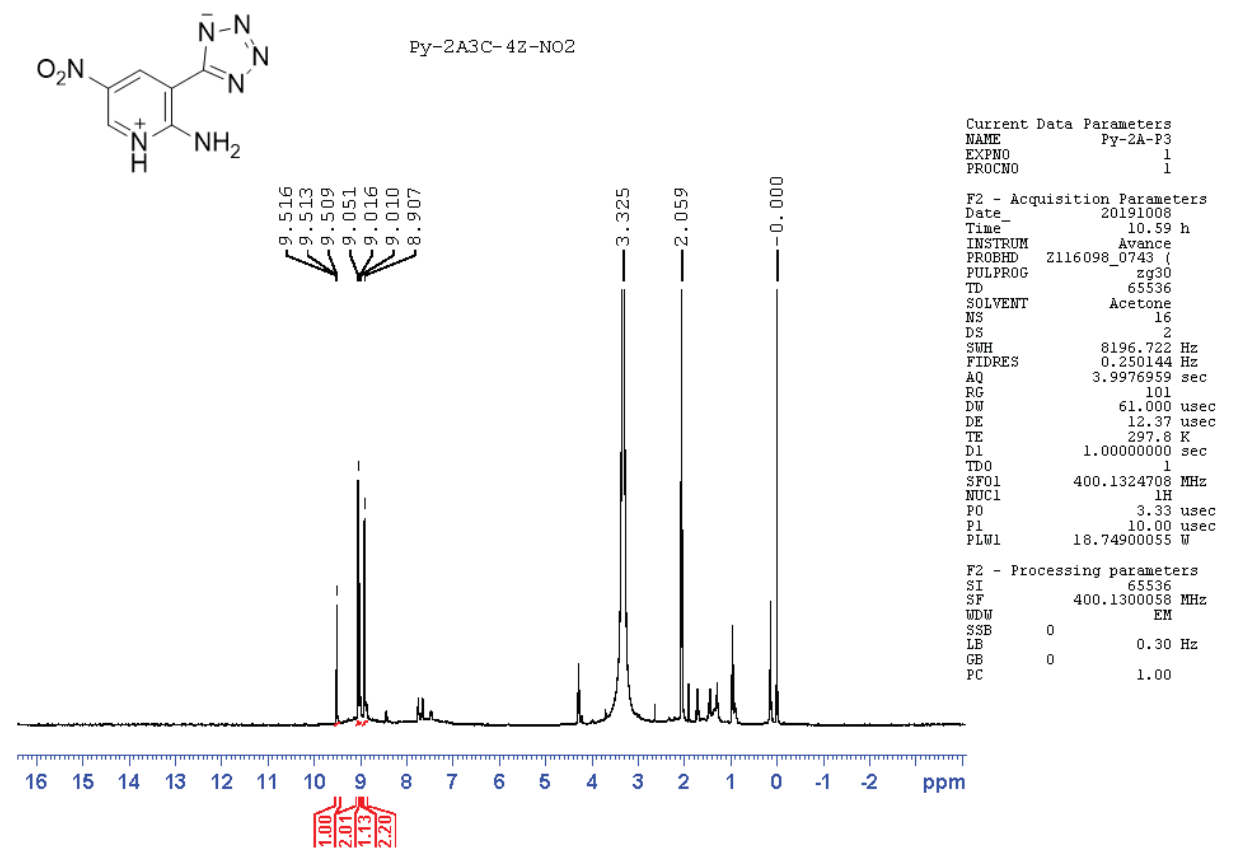

Figure $\mathrm{S} 45 .{ }^{1} \mathrm{H}$ NMR of $\mathbf{3 b - 2}$ in Acetone- $\mathrm{d}_{6}$ operating at $400.13 \mathrm{MHz}$.

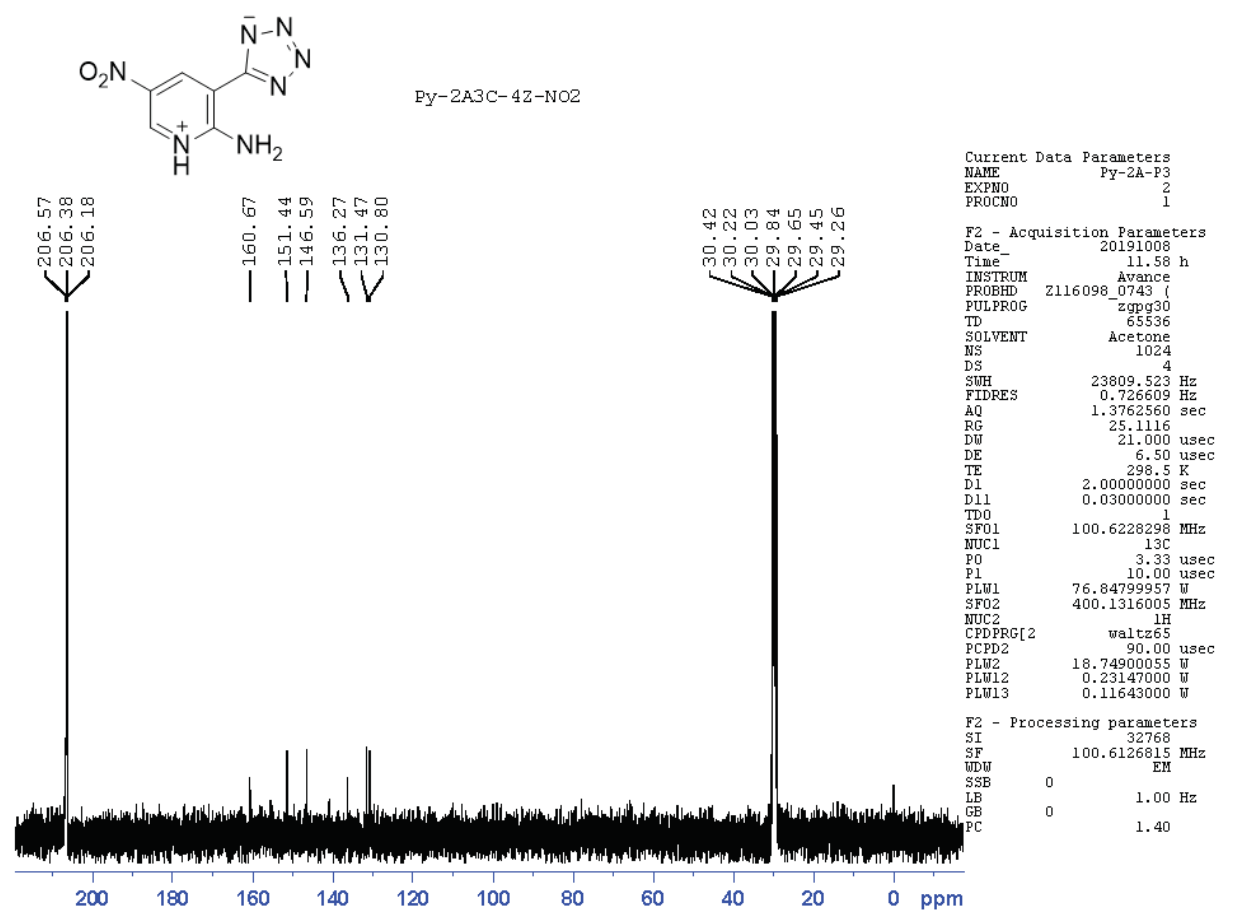

Figure S46. ${ }^{13} \mathrm{C}$ NMR of $\mathbf{3 b - 2}$ in Acetone- $\mathrm{d}_{6}$ operating at $100.61 \mathrm{MHz}$. 


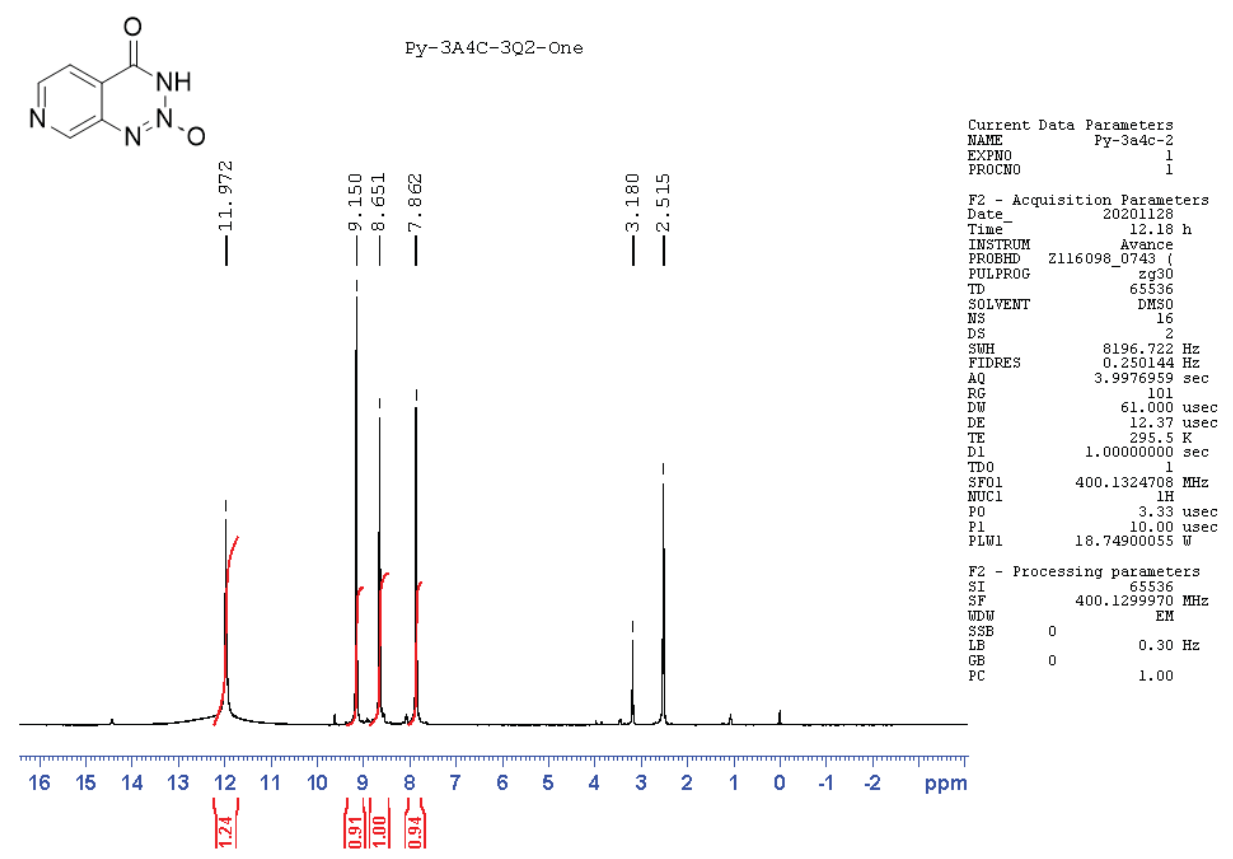

Figure S47. ${ }^{1} \mathrm{H}$ NMR of $3 \mathrm{c}-\mathbf{1}$ in DMSO- $\mathrm{d}_{6}$ operating at $400.13 \mathrm{MHz}$.

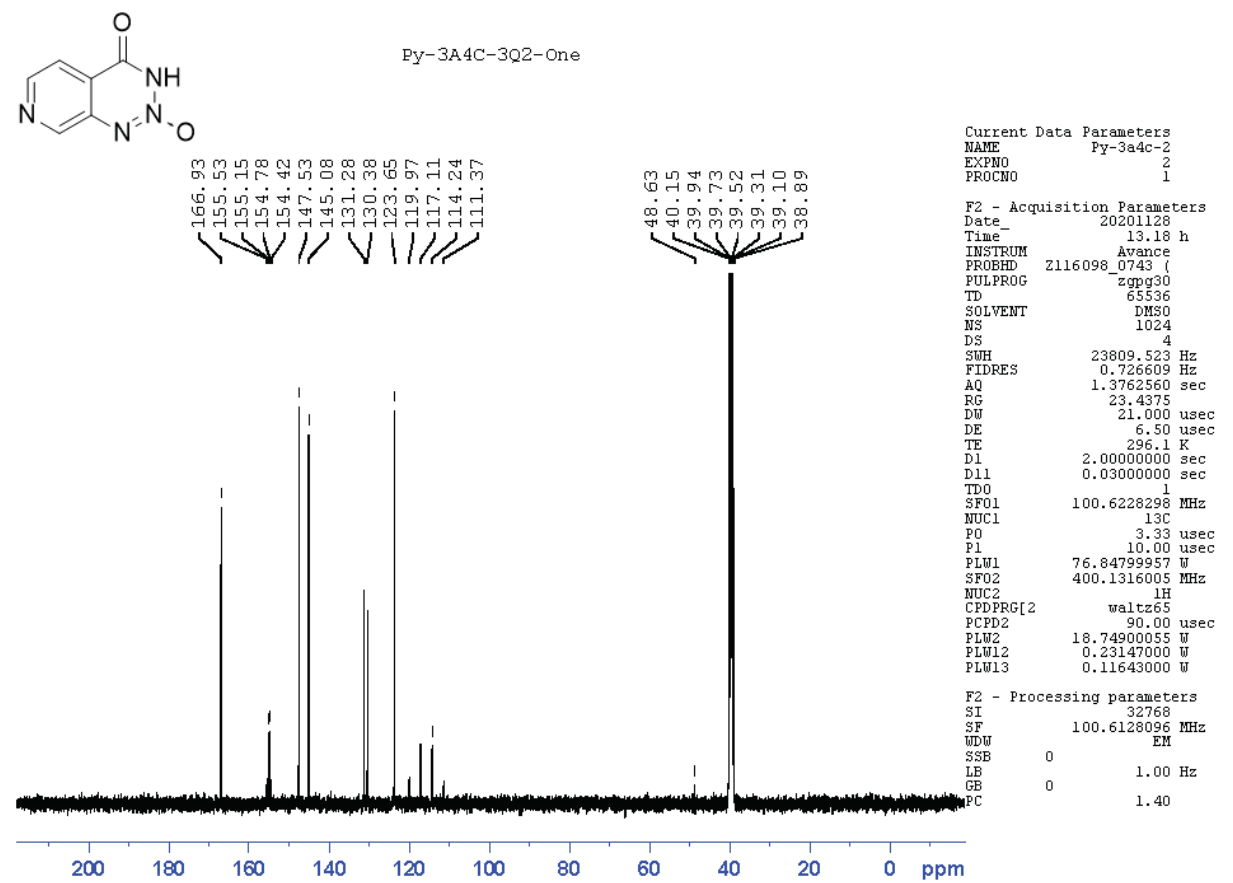

Figure S48. ${ }^{13} \mathrm{C}$ NMR of $\mathbf{3 c}-\mathbf{1}$ in DMSO- $\mathrm{d}_{6}$ operating at $100.61 \mathrm{MHz}$. 


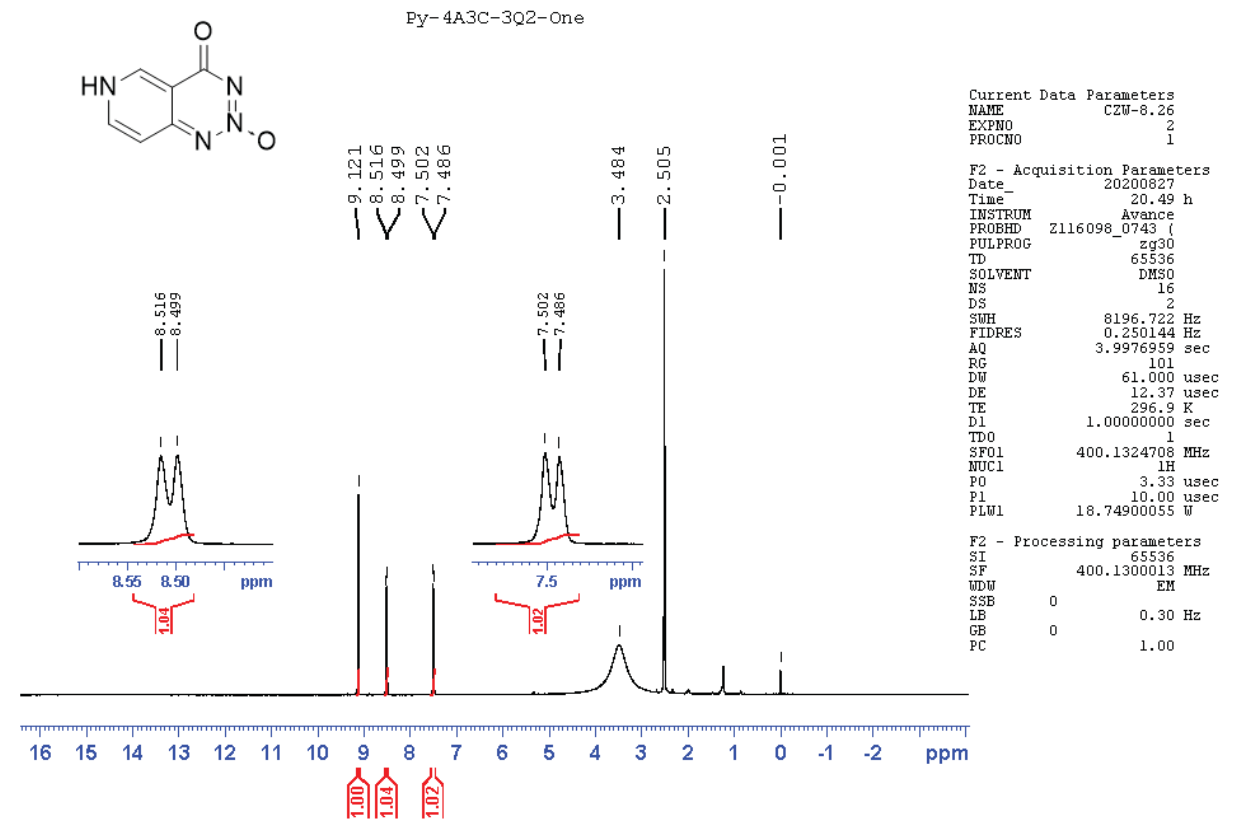

Figure S49. ${ }^{1} \mathrm{H}$ NMR of $\mathbf{3 d - 1}$ in DMSO-d $\mathrm{d}_{6}$ operating at $400.13 \mathrm{MHz}$.

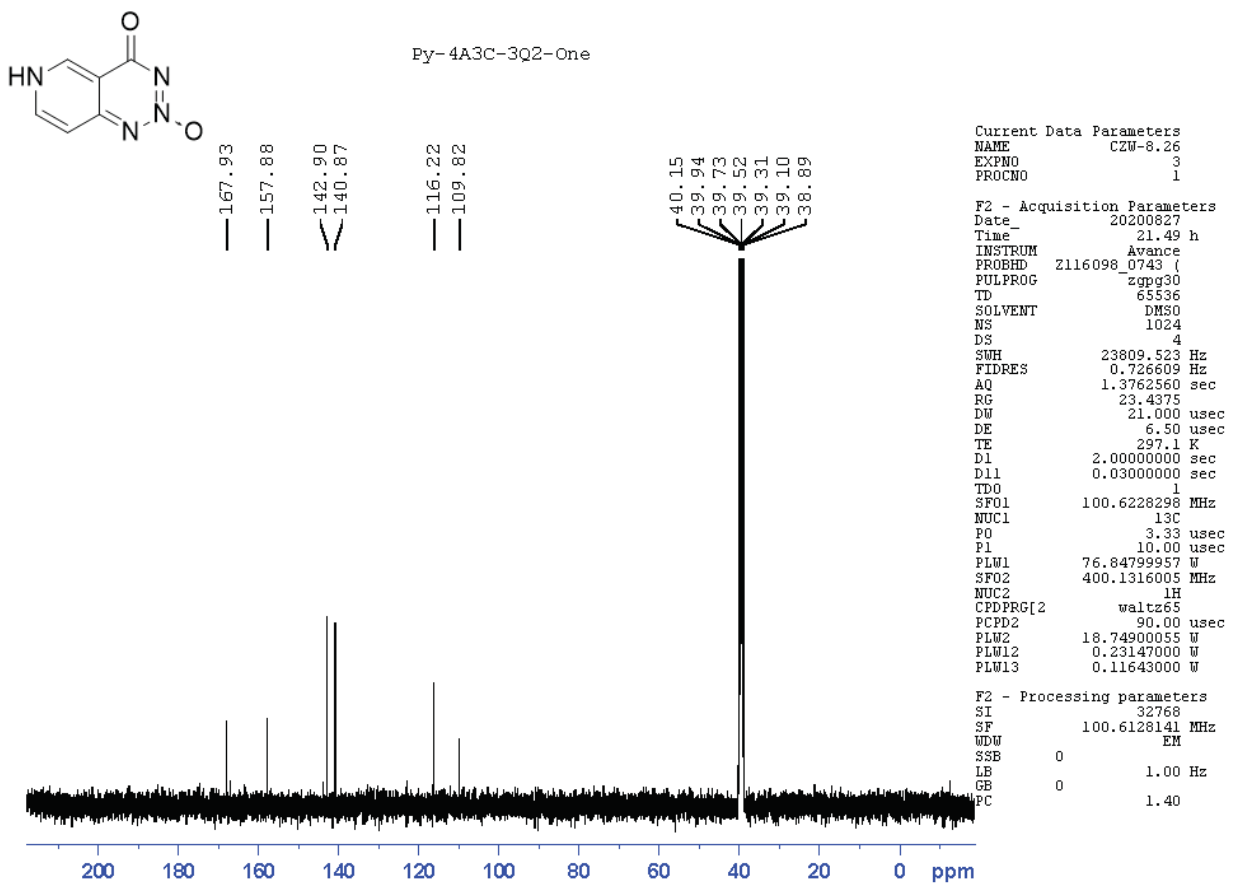

Figure S50. ${ }^{13} \mathrm{C}$ NMR of $\mathbf{3 d - 1}$ in DMSO- $\mathrm{d}_{6}$ operating at $100.61 \mathrm{MHz}$. 


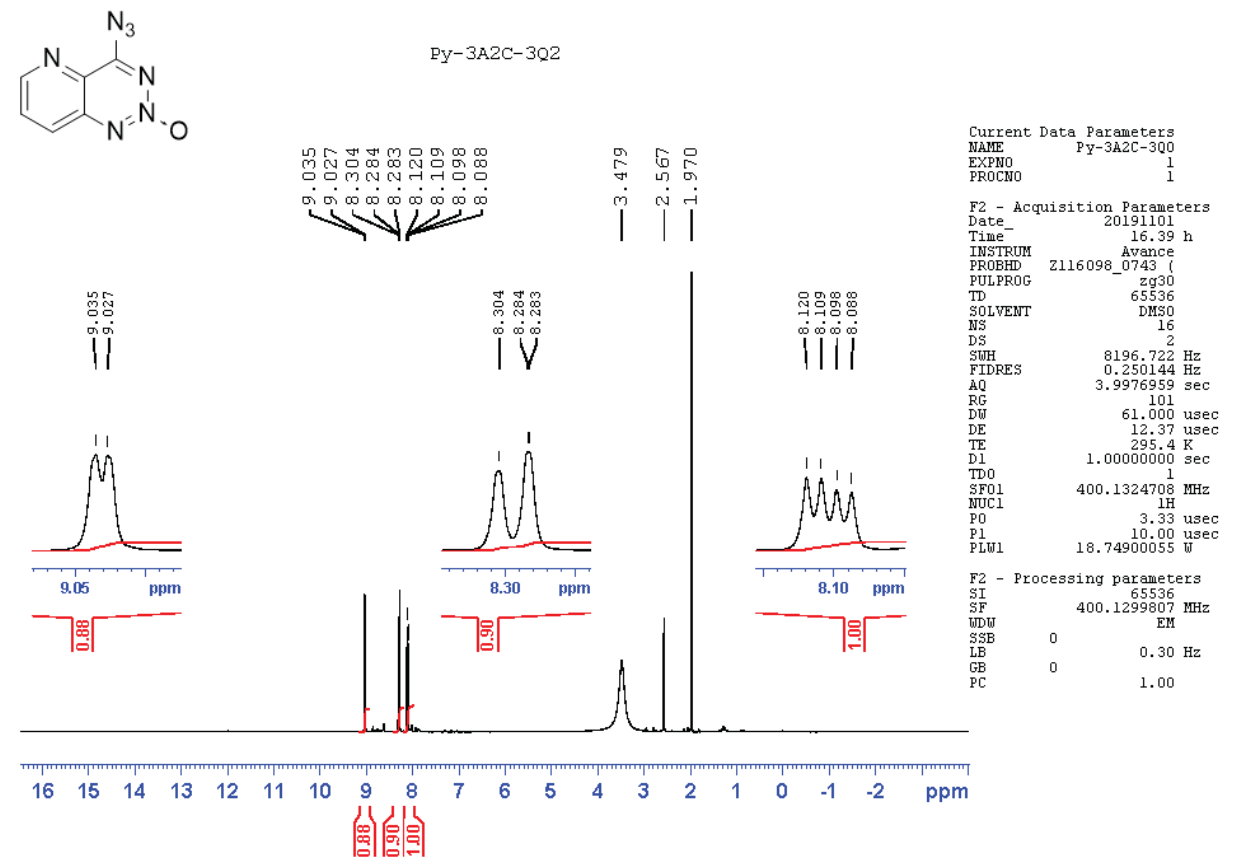

Figure $\mathrm{S} 51 .{ }^{1} \mathrm{H}$ NMR of $3 \mathrm{e}$ in DMSO- $\mathrm{d}_{6}$ operating at $400.13 \mathrm{MHz}$.

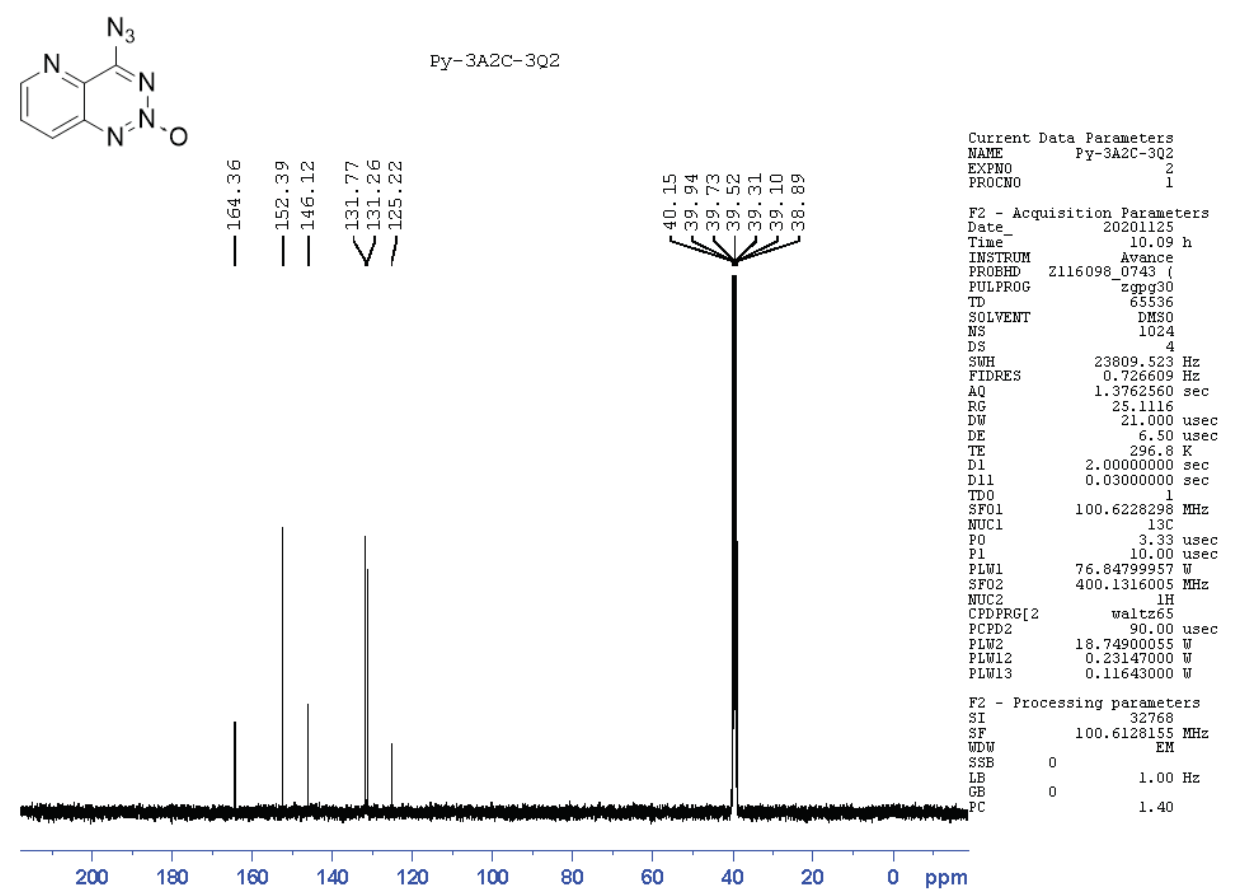

Figure $552 .{ }^{13} \mathrm{C}$ NMR of $3 e$ in DMSO- $\mathrm{d}_{6}$ operating at $100.61 \mathrm{MHz}$. 


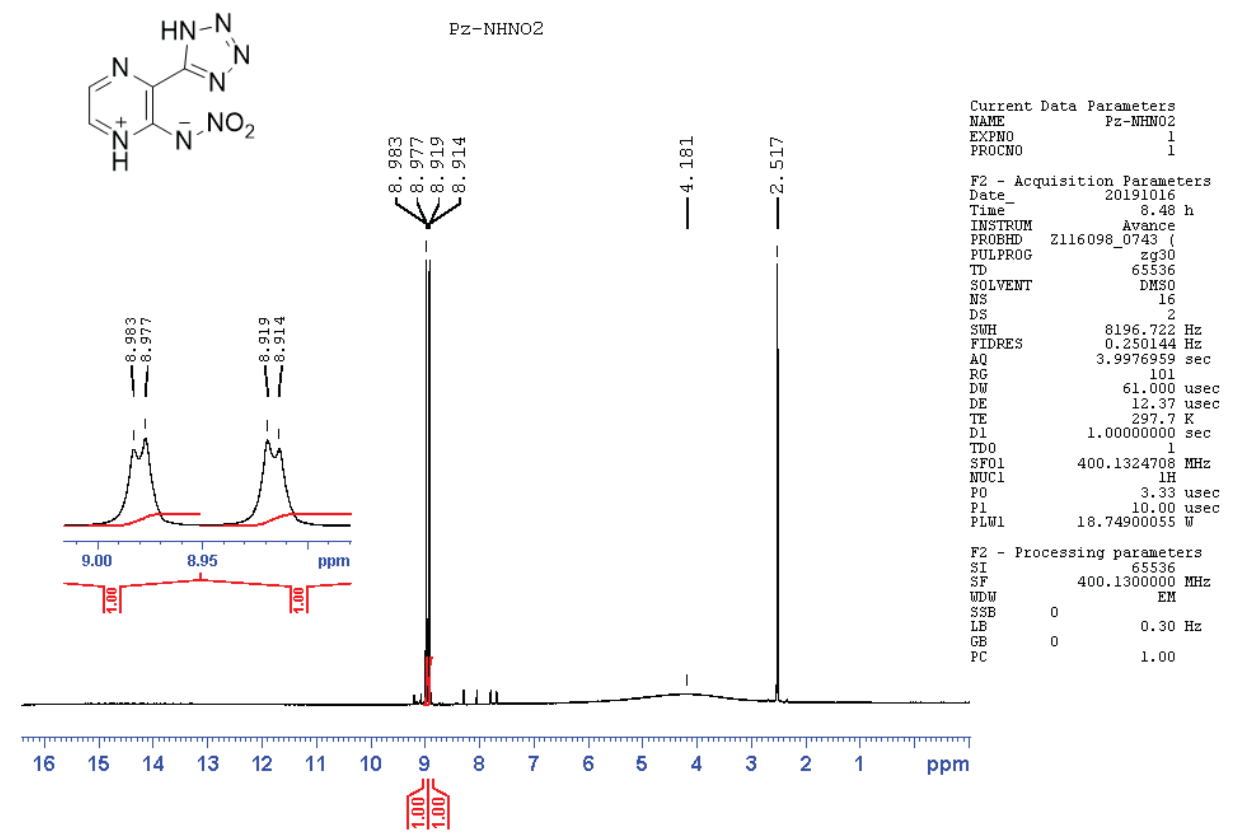

Figure $\mathrm{S} 53 .{ }^{1} \mathrm{H}$ NMR of $\mathbf{3 f - 1}$ in DMSO- $\mathrm{d}_{6}$ operating at $400.13 \mathrm{MHz}$.

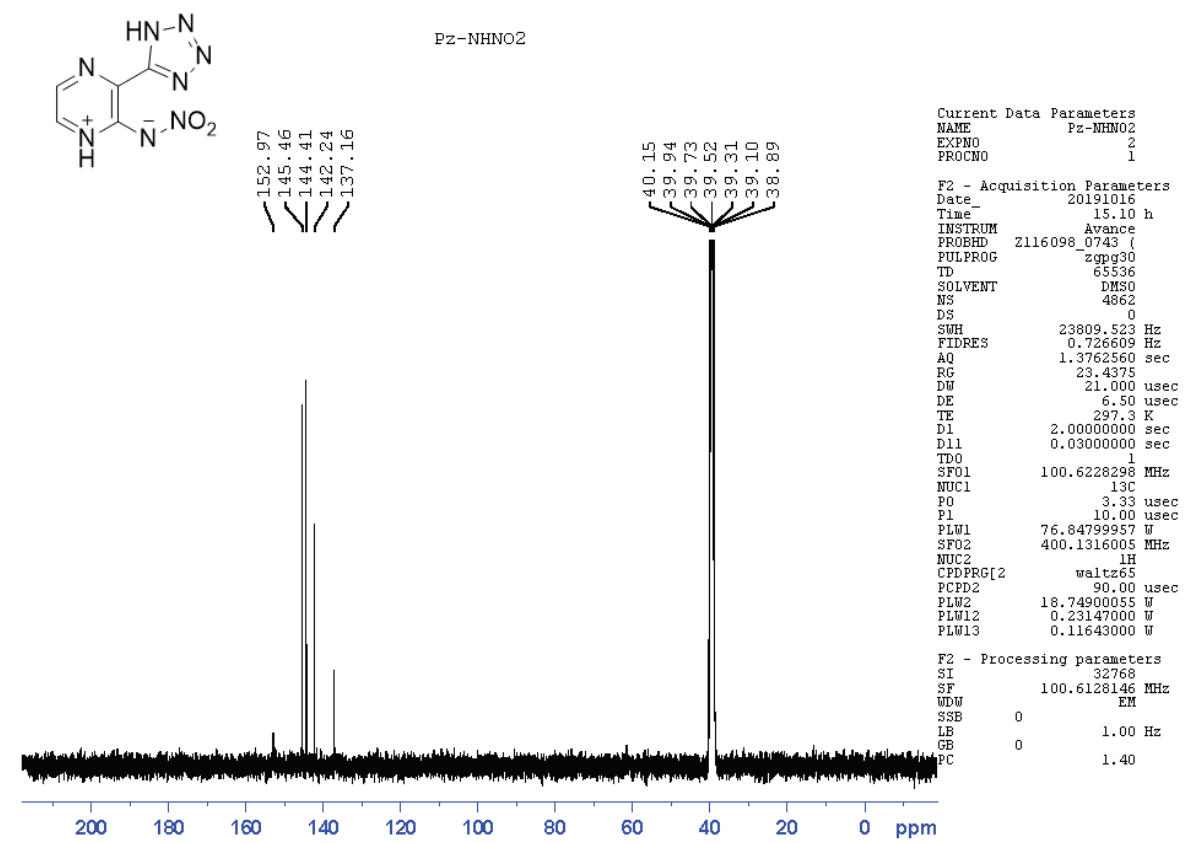

Figure S54. ${ }^{13} \mathrm{C}$ NMR of $\mathbf{3 f - 1}$ in DMSO- $\mathrm{d}_{6}$ operating at $100.61 \mathrm{MHz}$. 


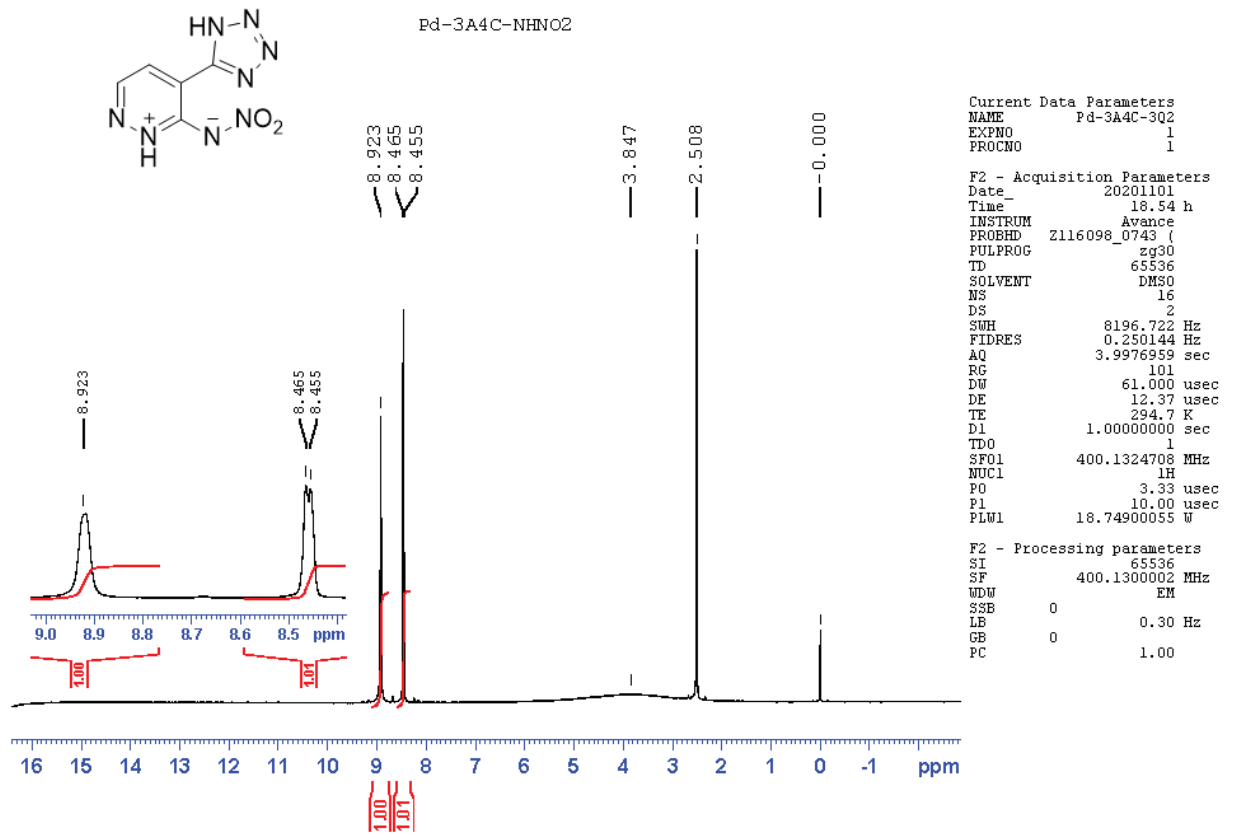

Figure S55. ${ }^{1} \mathrm{H}$ NMR of $\mathbf{3 g - 1}$ in DMSO- $\mathrm{d}_{6}$ operating at $400.13 \mathrm{MHz}$.

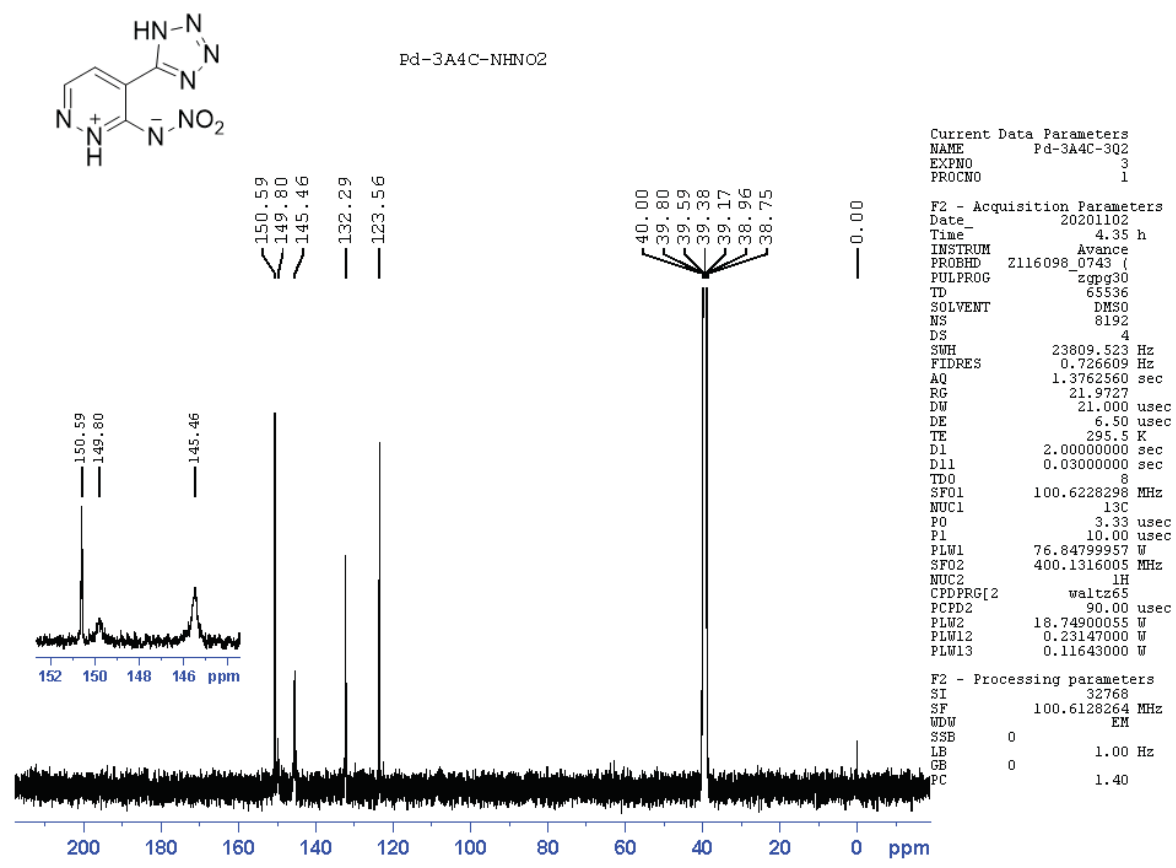

Figure S56. ${ }^{13} \mathrm{C}$ NMR of $3 g-1$ in DMSO- $d_{6}$ operating at $100.61 \mathrm{MHz}$. 


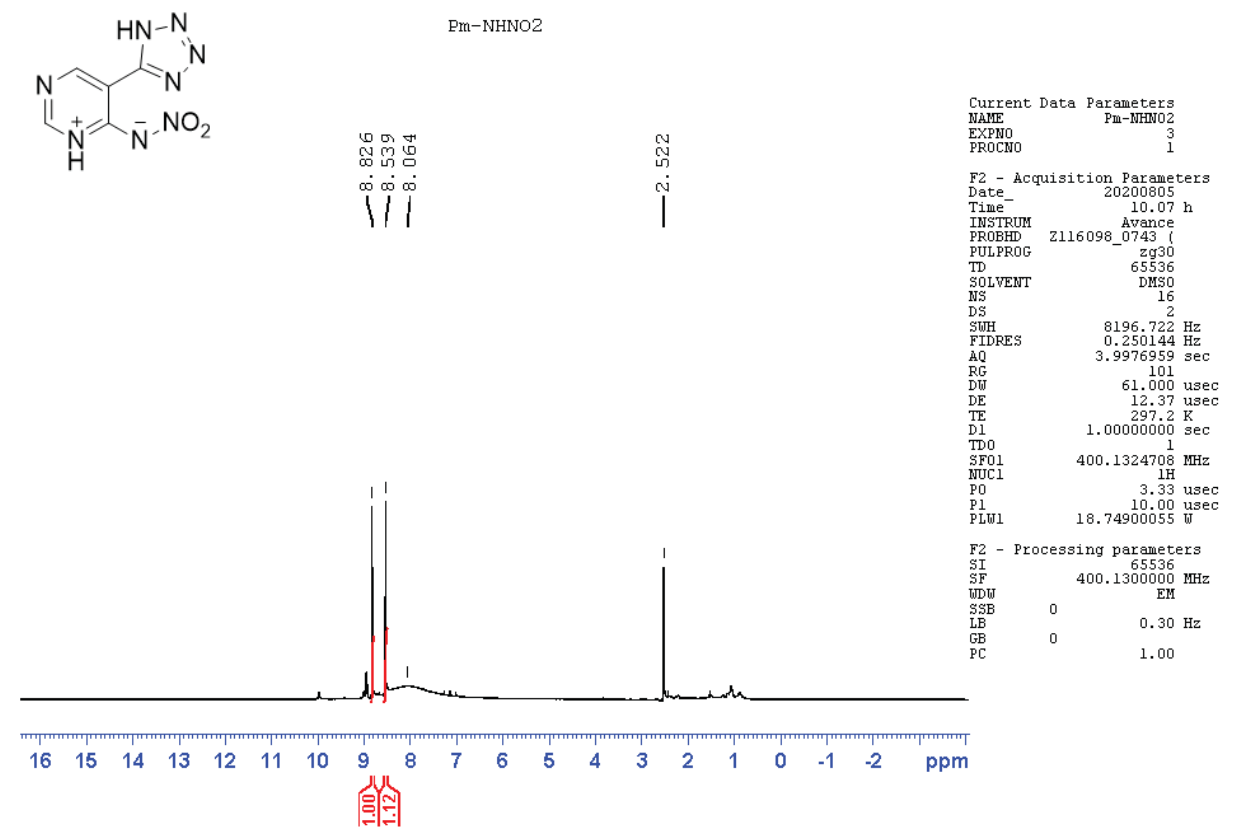

Figure S57. ${ }^{1} \mathrm{H}$ NMR of $\mathbf{3 h - 1}$ in DMSO-d 6 operating at $400.13 \mathrm{MHz}$.

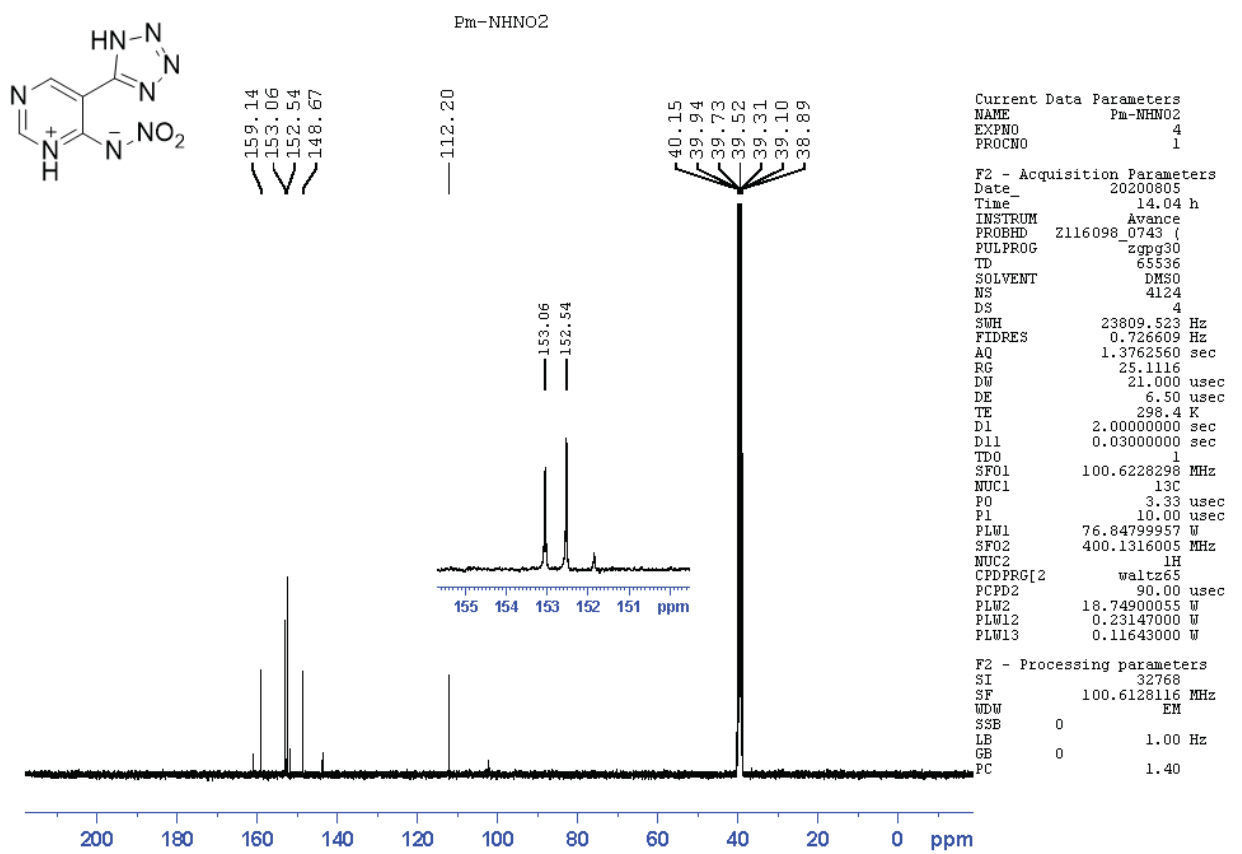

Figure S58. ${ }^{13} \mathrm{C}$ NMR of $\mathbf{3 h - 1}$ in DMSO-d $\mathrm{d}_{6}$ operating at $100.61 \mathrm{MHz}$. 


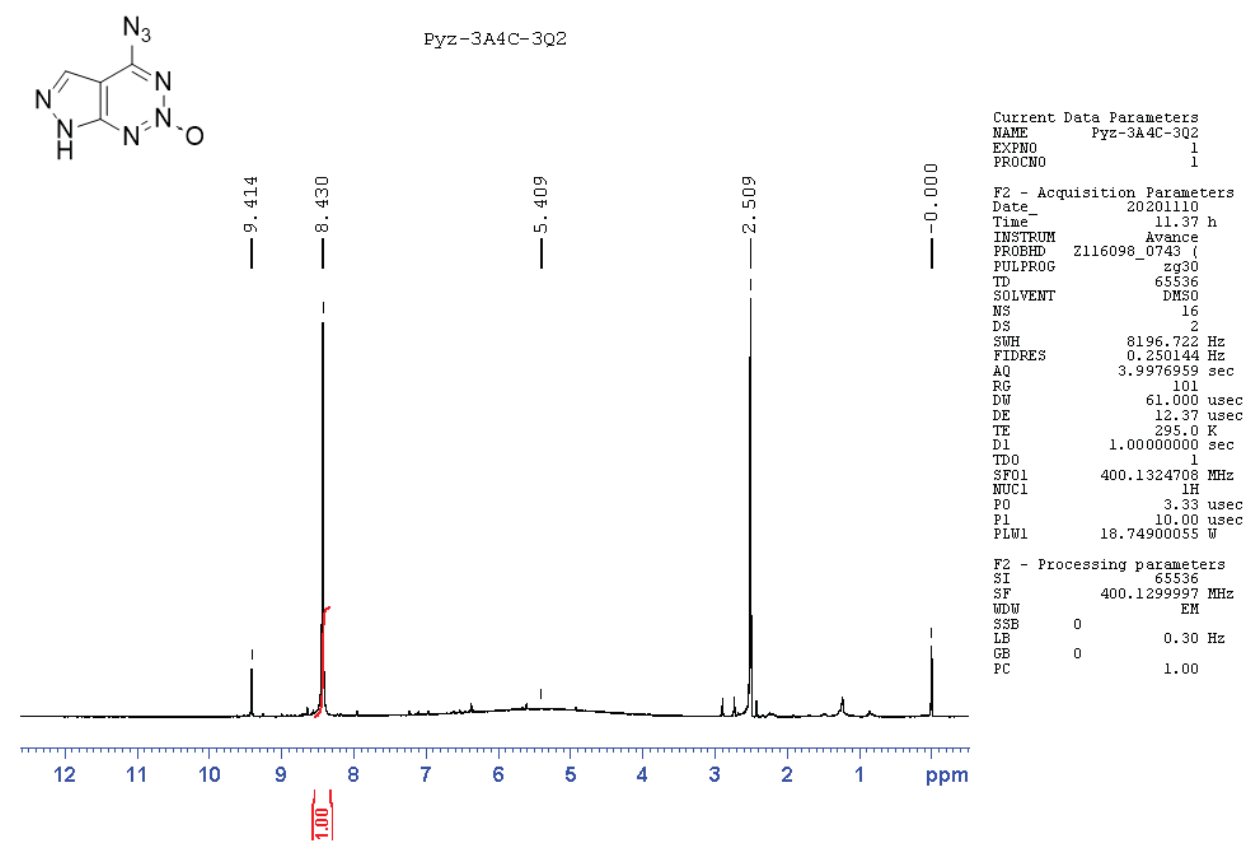

Figure S59. ${ }^{1} \mathrm{H}$ NMR of $3 \mathbf{i}$ in DMSO- $\mathrm{d}_{6}$ operating at $400.13 \mathrm{MHz}$.

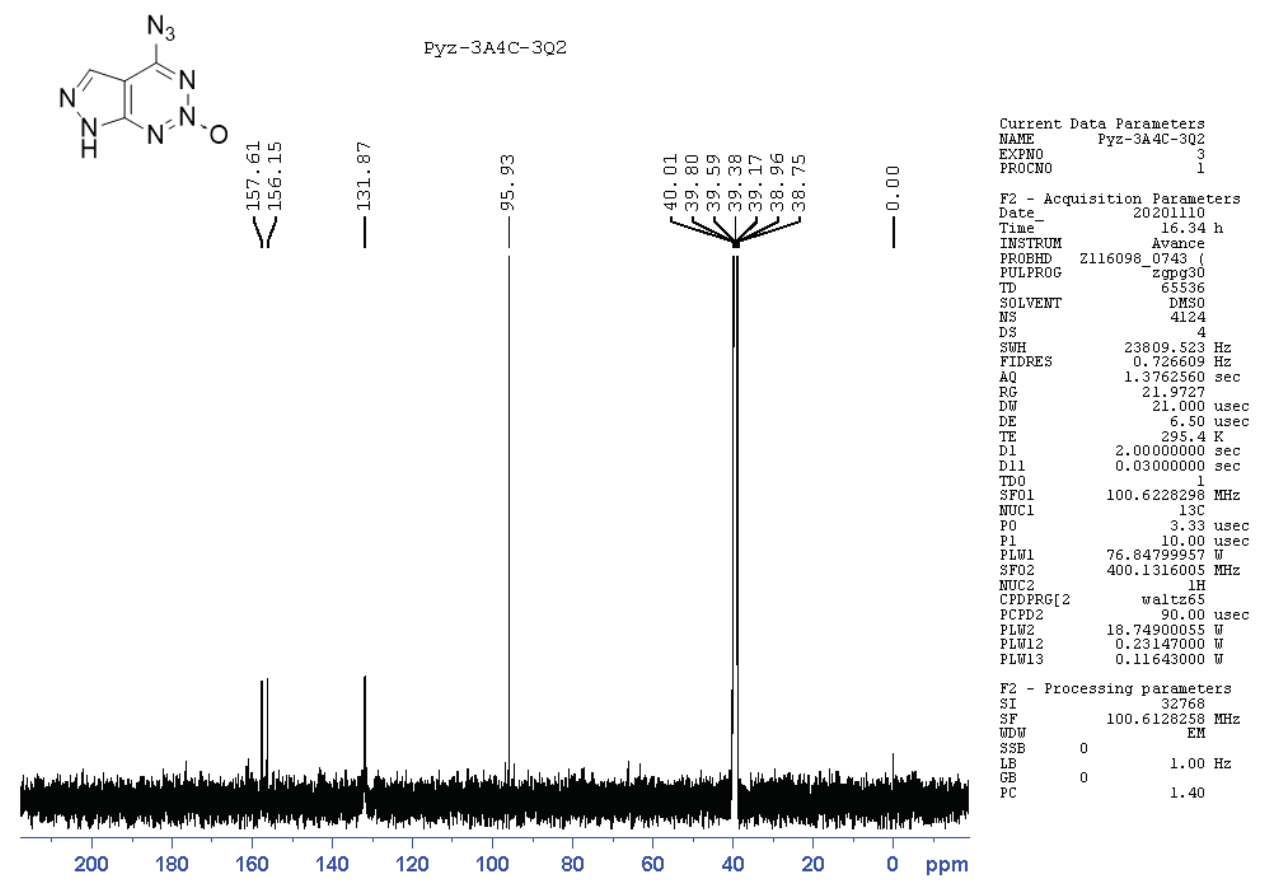

Figure S60. ${ }^{13} \mathrm{C}$ NMR of $3 \mathbf{i}$ in DMSO- $\mathrm{d}_{6}$ operating at $100.61 \mathrm{MHz}$. 


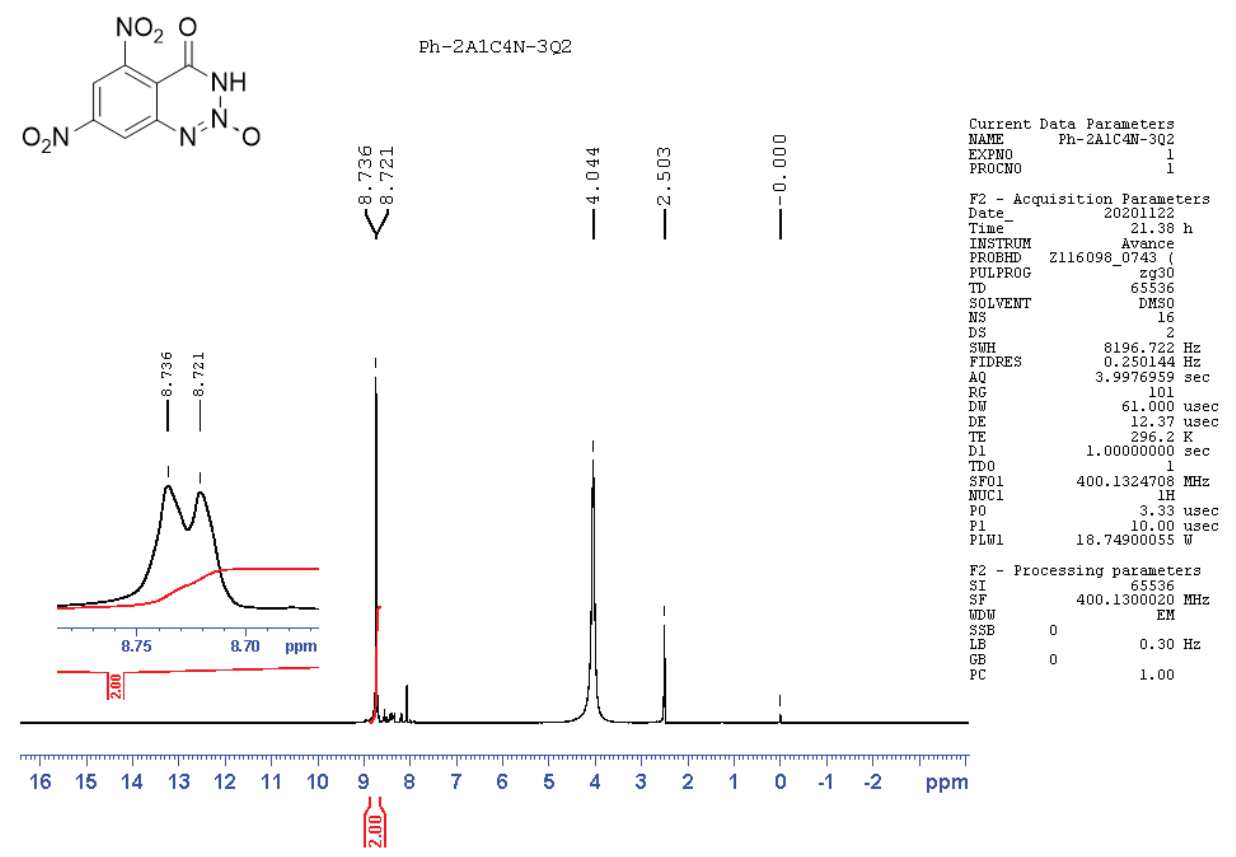

Figure S61. ${ }^{1} \mathrm{H}$ NMR of $3 \mathrm{I}-1$ in DMSO- $\mathrm{d}_{6}$ operating at $400.13 \mathrm{MHz}$.

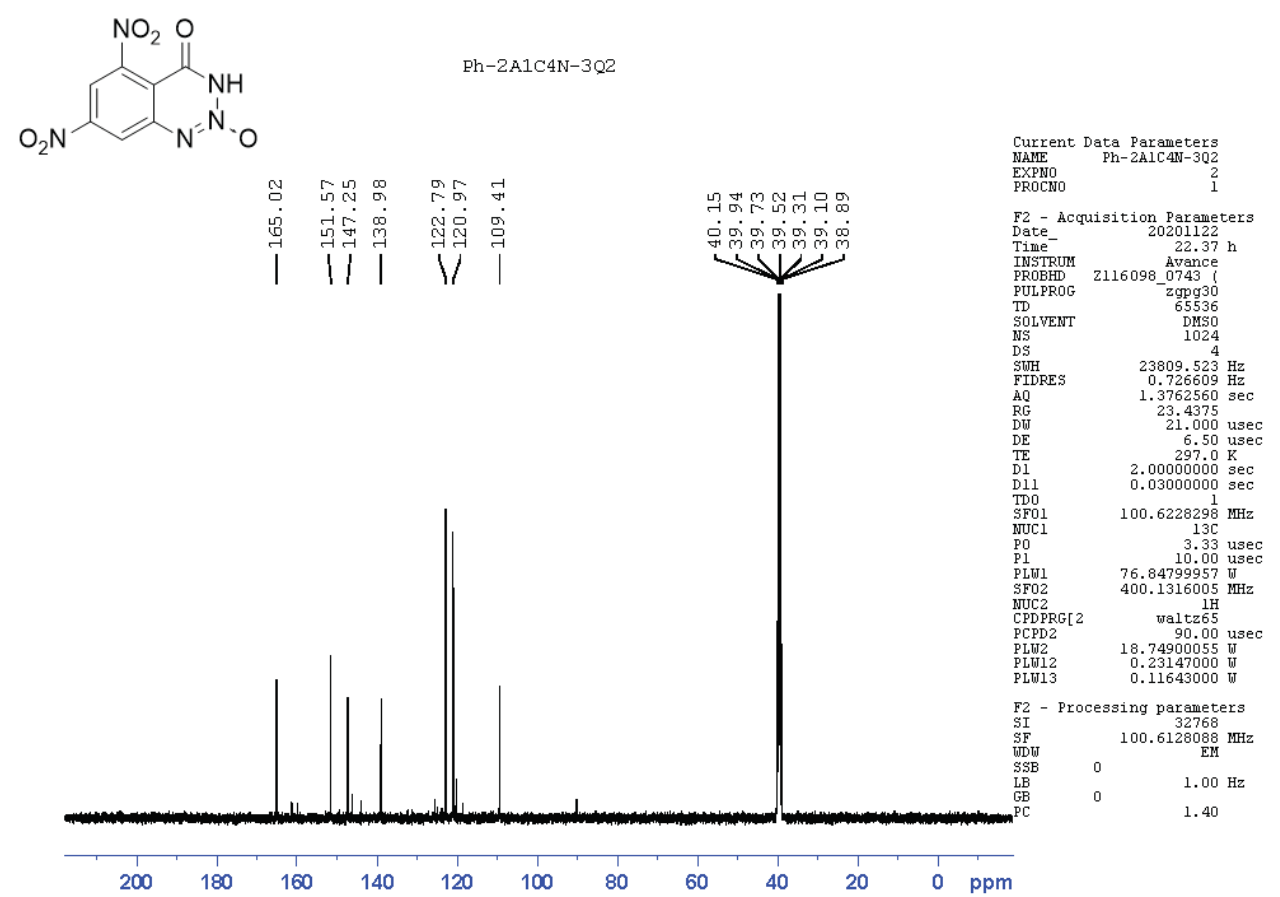

Figure S62. ${ }^{13} \mathrm{C}$ NMR of $3 \mathrm{I}-1$ in DMSO-d 6 operating at $100.61 \mathrm{MHz}$. 


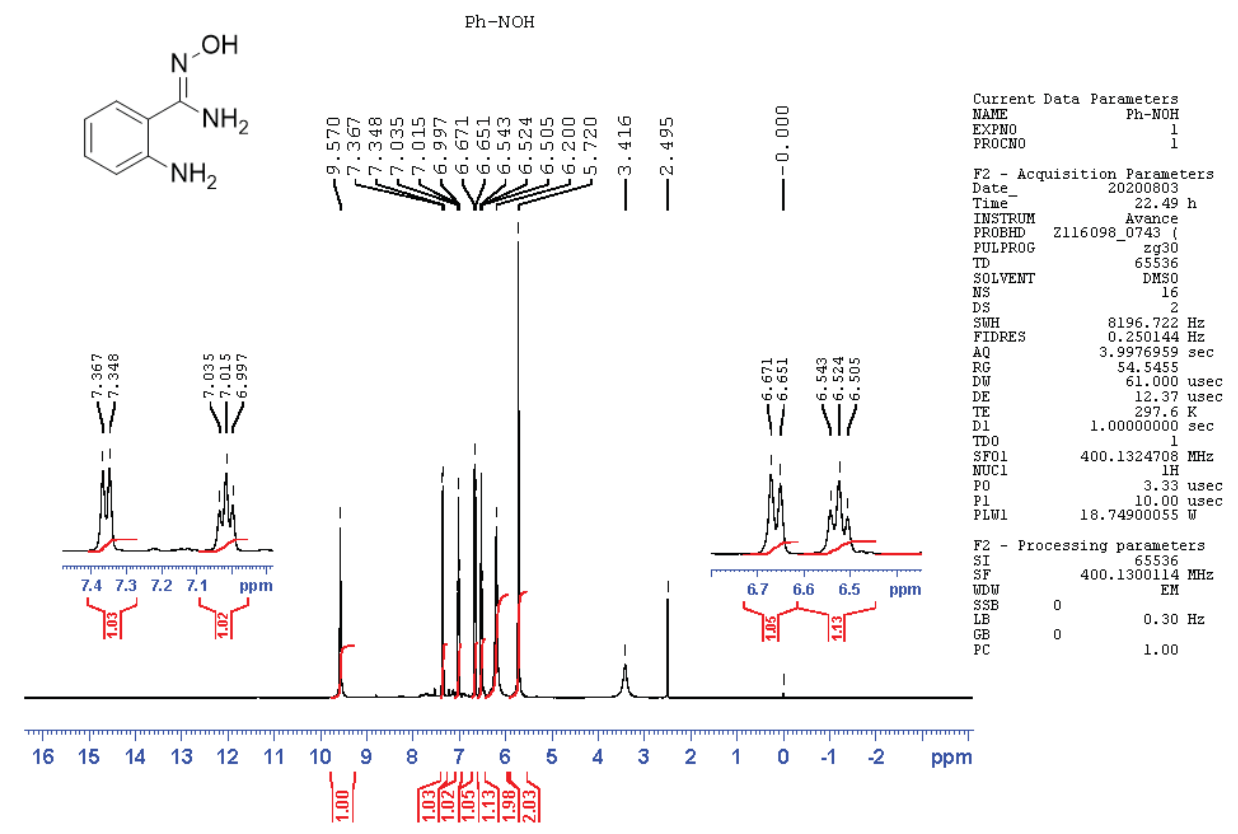

Figure S63. ${ }^{1} \mathrm{H}$ NMR of 4 a in DMSO-d 6 operating at $400.13 \mathrm{MHz}$.

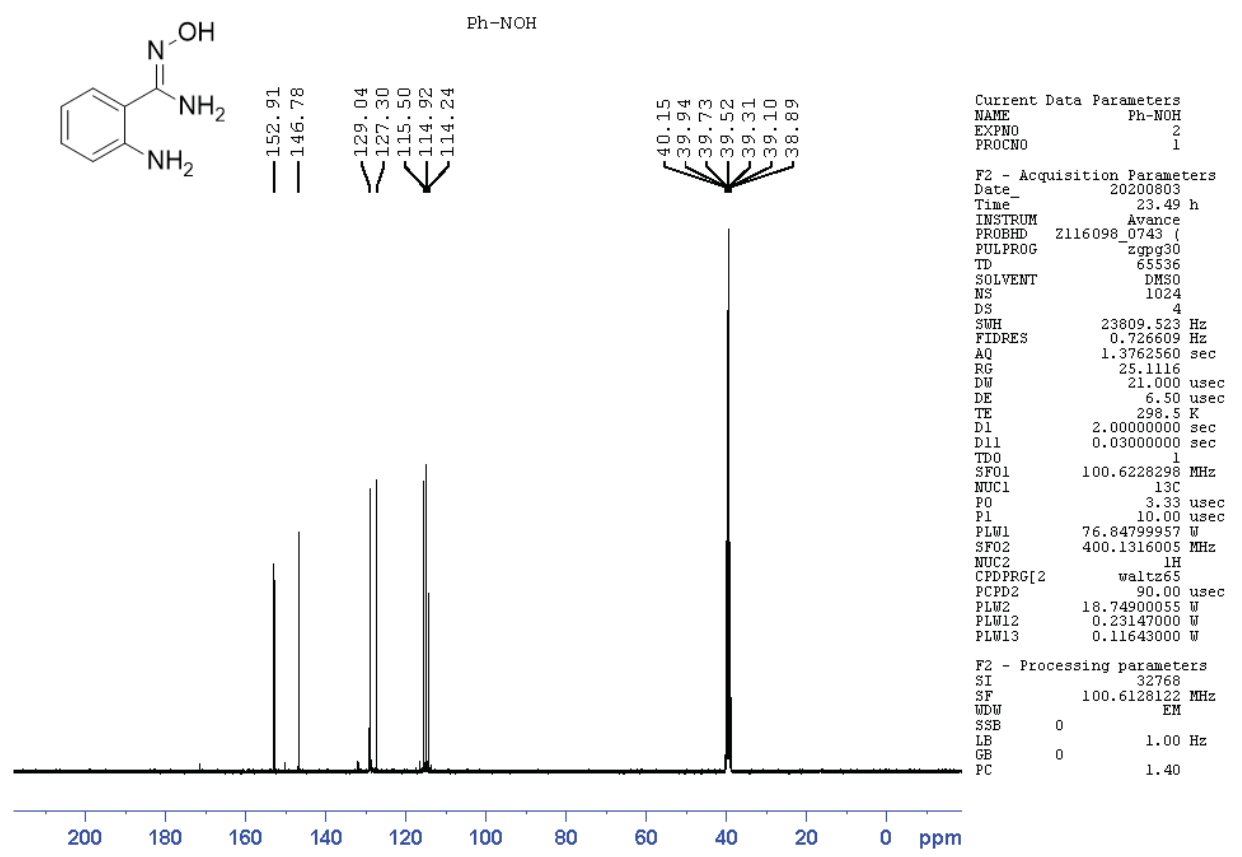

Figure $564 .{ }^{13} \mathrm{C}$ NMR of $4 a$ in DMSO- $\mathrm{d}_{6}$ operating at $100.61 \mathrm{MHz}$. 


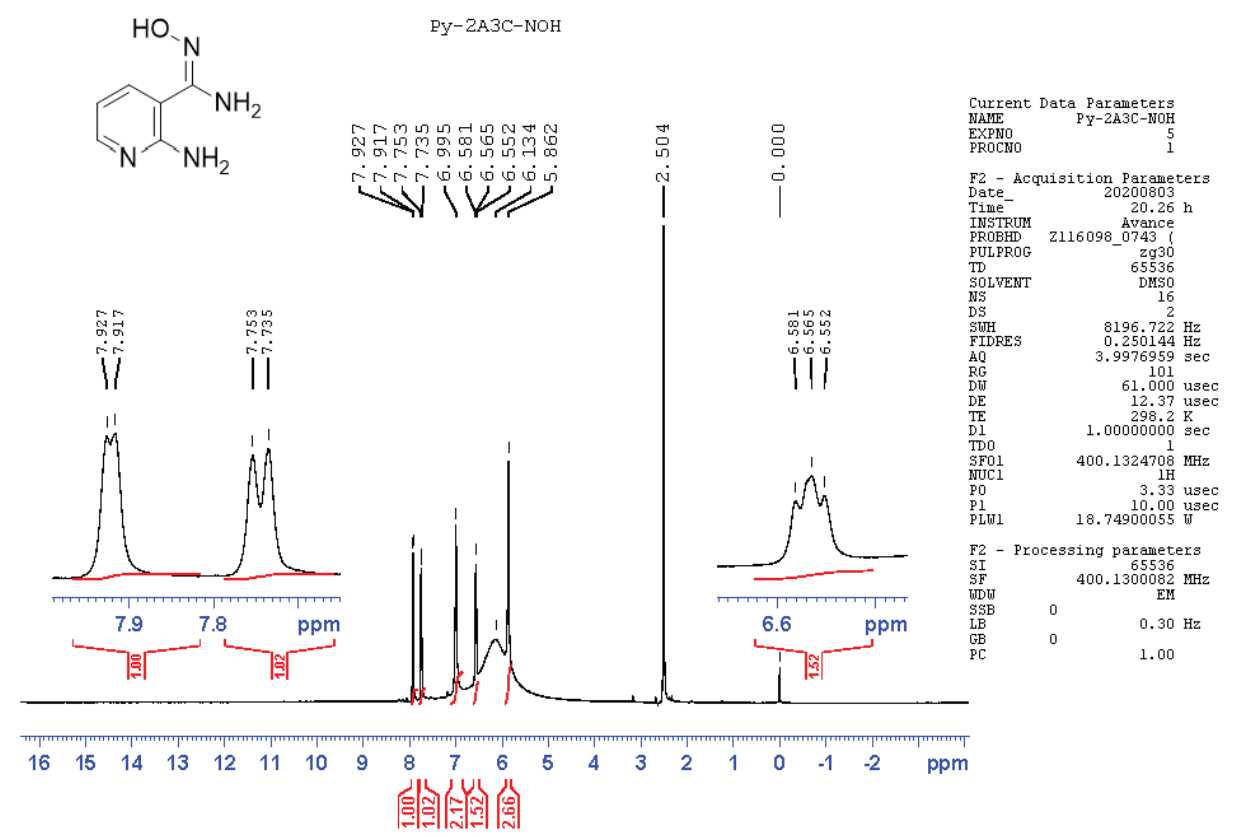

Figure $\mathrm{S} 65 .{ }^{1} \mathrm{H}$ NMR of $\mathbf{4 b}$ in DMSO-d $\mathrm{d}_{6}$ operating at $400.13 \mathrm{MHz}$.

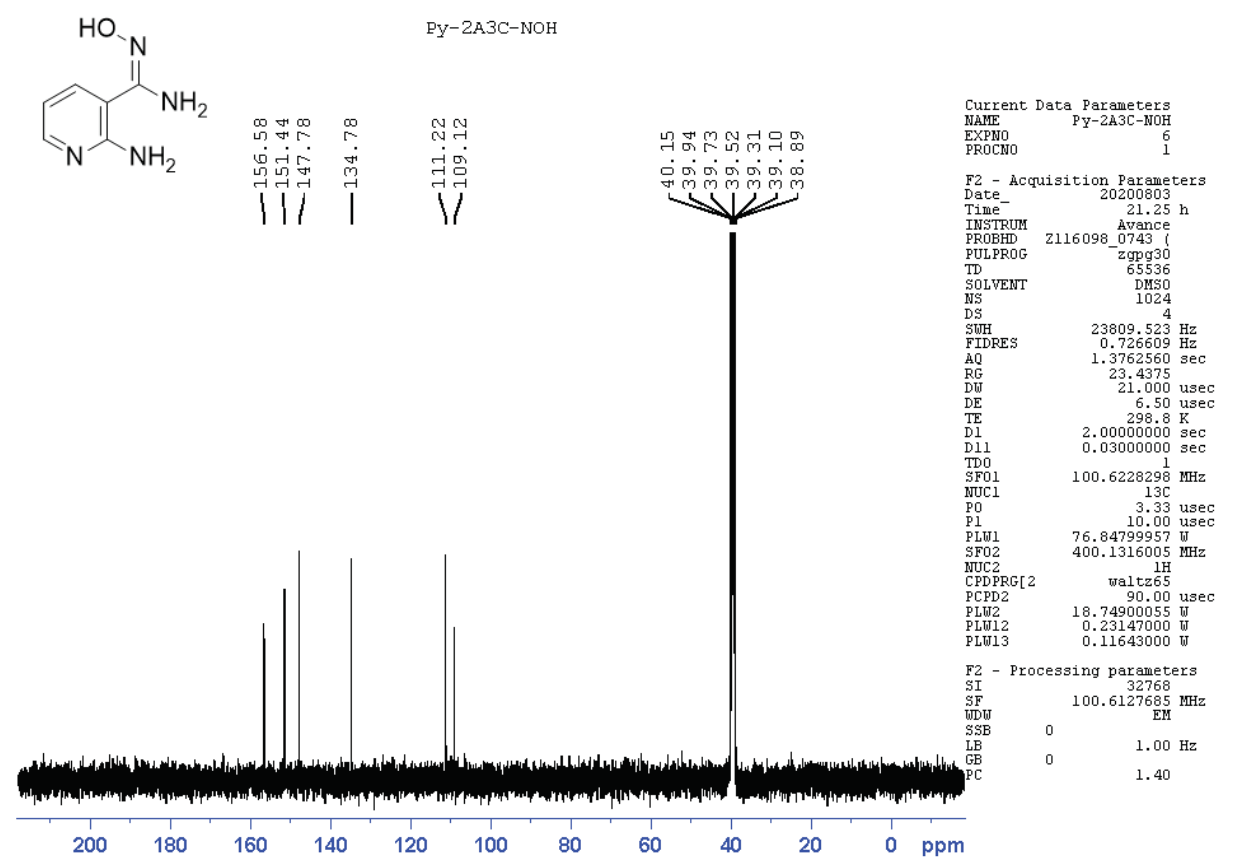

Figure S66. ${ }^{13} \mathrm{C}$ NMR of $4 b$ in DMSO- $d_{6}$ operating at $100.61 \mathrm{MHz}$. 


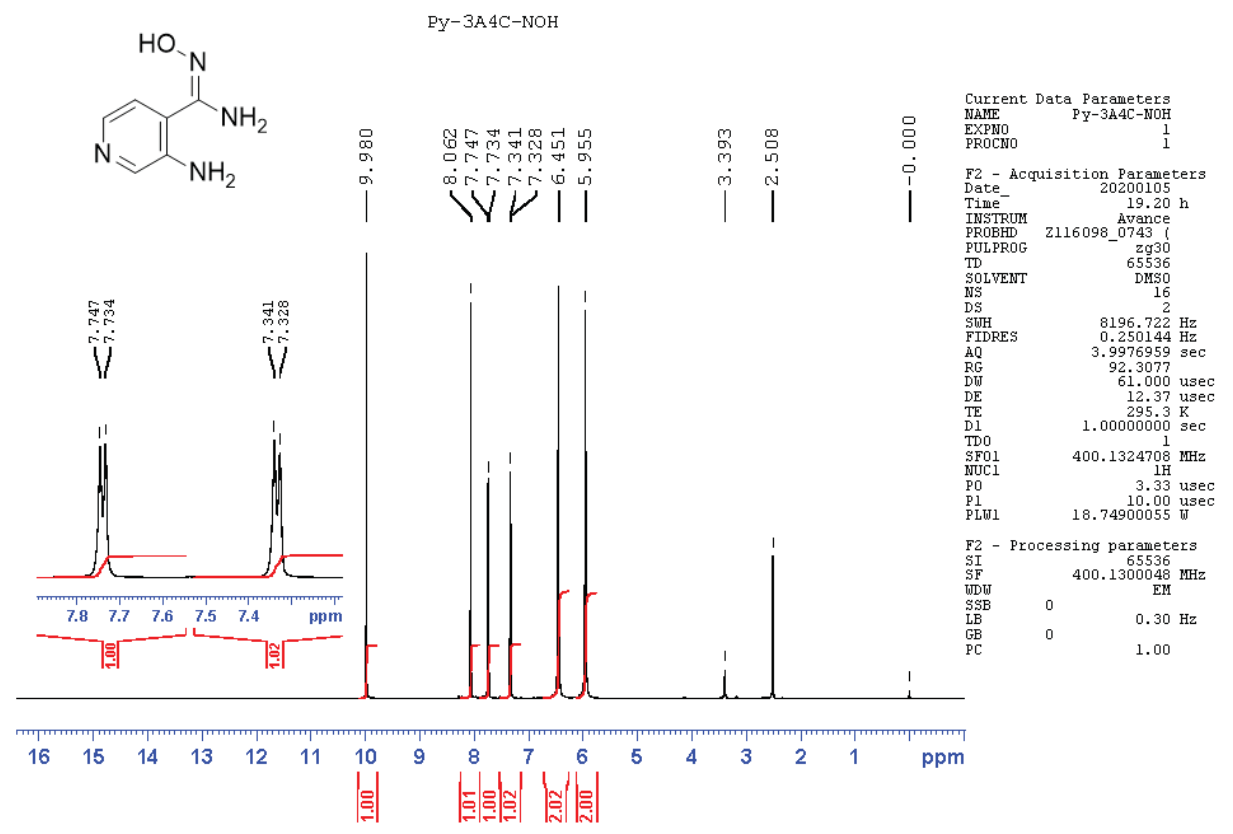

Figure S67. ${ }^{1} \mathrm{H}$ NMR of $4 \mathrm{c}$ in DMSO- $\mathrm{d}_{6}$ operating at $400.13 \mathrm{MHz}$.

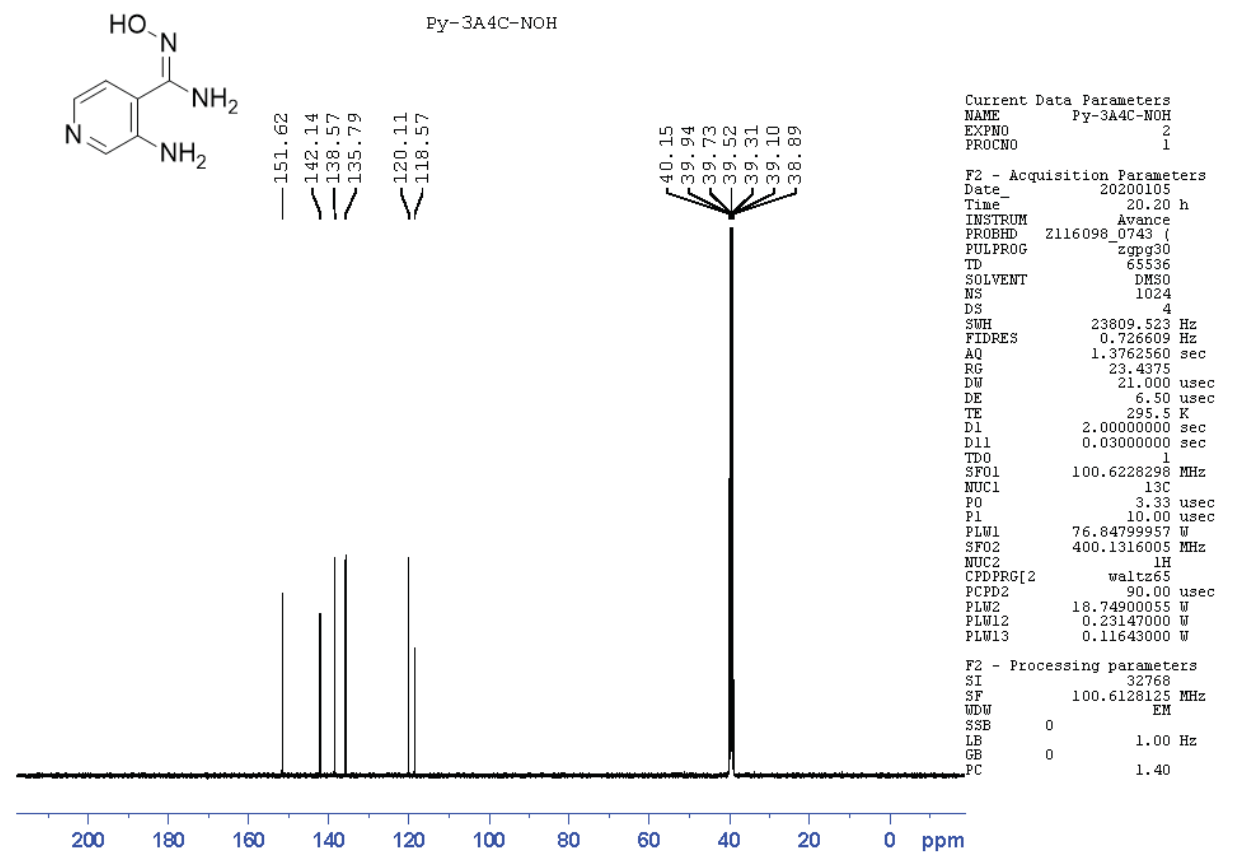

Figure S68. ${ }^{13} \mathrm{C}$ NMR of $4 \mathrm{c}$ in DMSO- $\mathrm{d}_{6}$ operating at $100.61 \mathrm{MHz}$. 


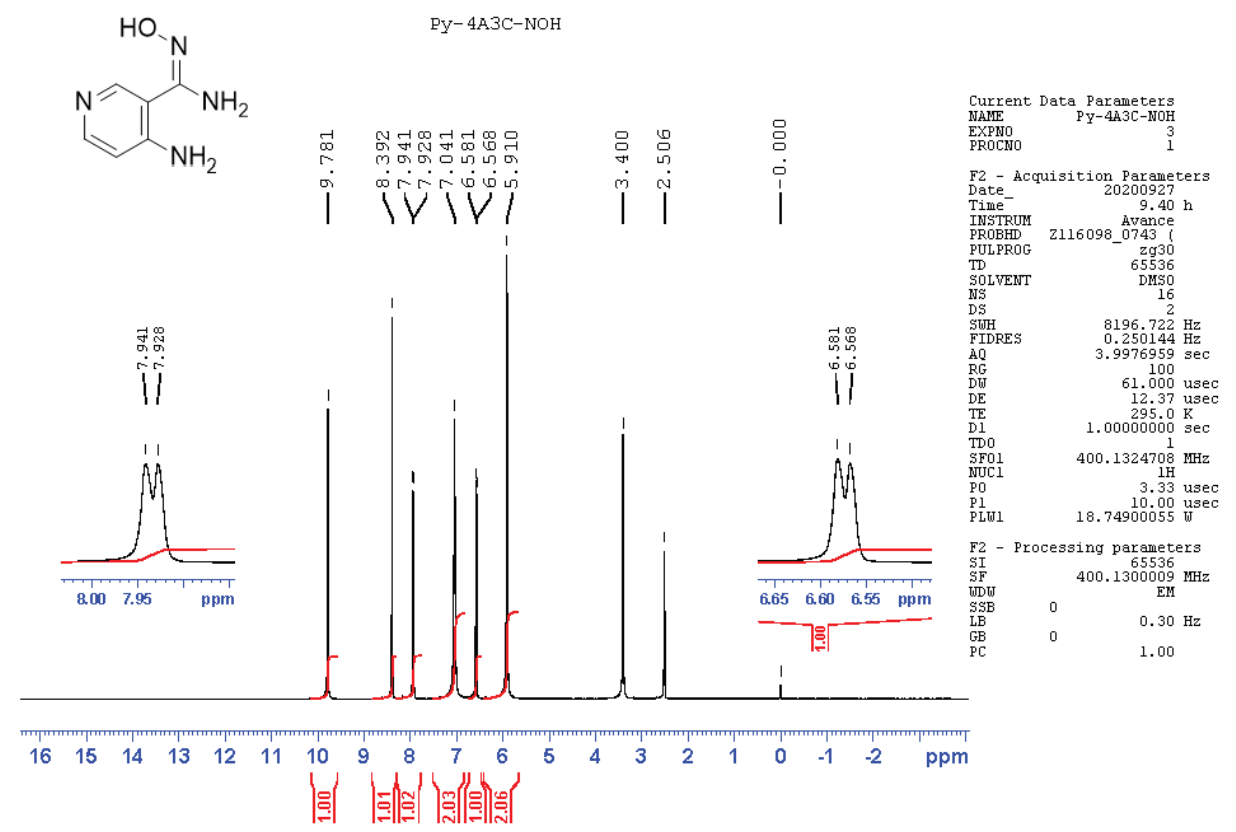

Figure S69. ${ }^{1} \mathrm{H}$ NMR of $4 \mathrm{~d}$ in DMSO- $\mathrm{d}_{6}$ operating at $400.13 \mathrm{MHz}$.

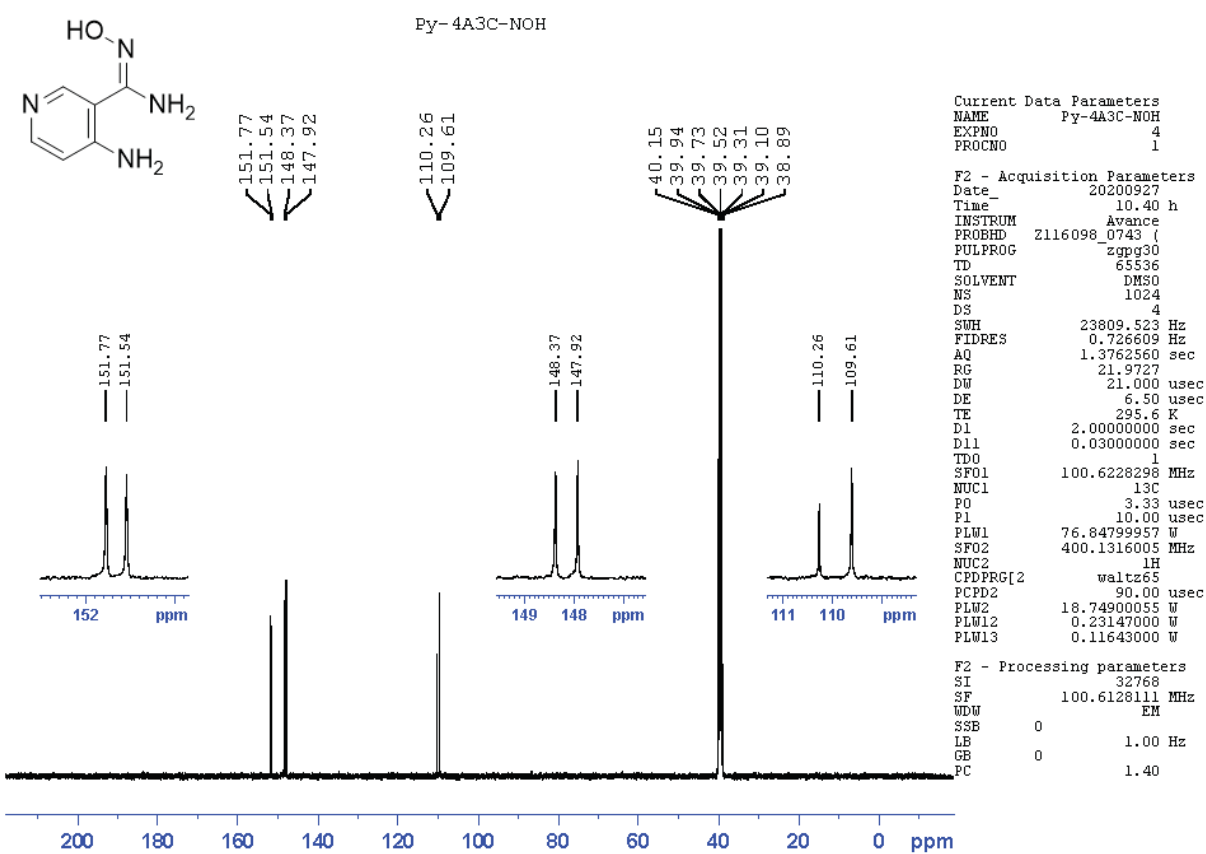

Figure S70. ${ }^{13} \mathrm{C}$ NMR of $4 d$ in DMSO- $\mathrm{d}_{6}$ operating at $100.61 \mathrm{MHz}$. 


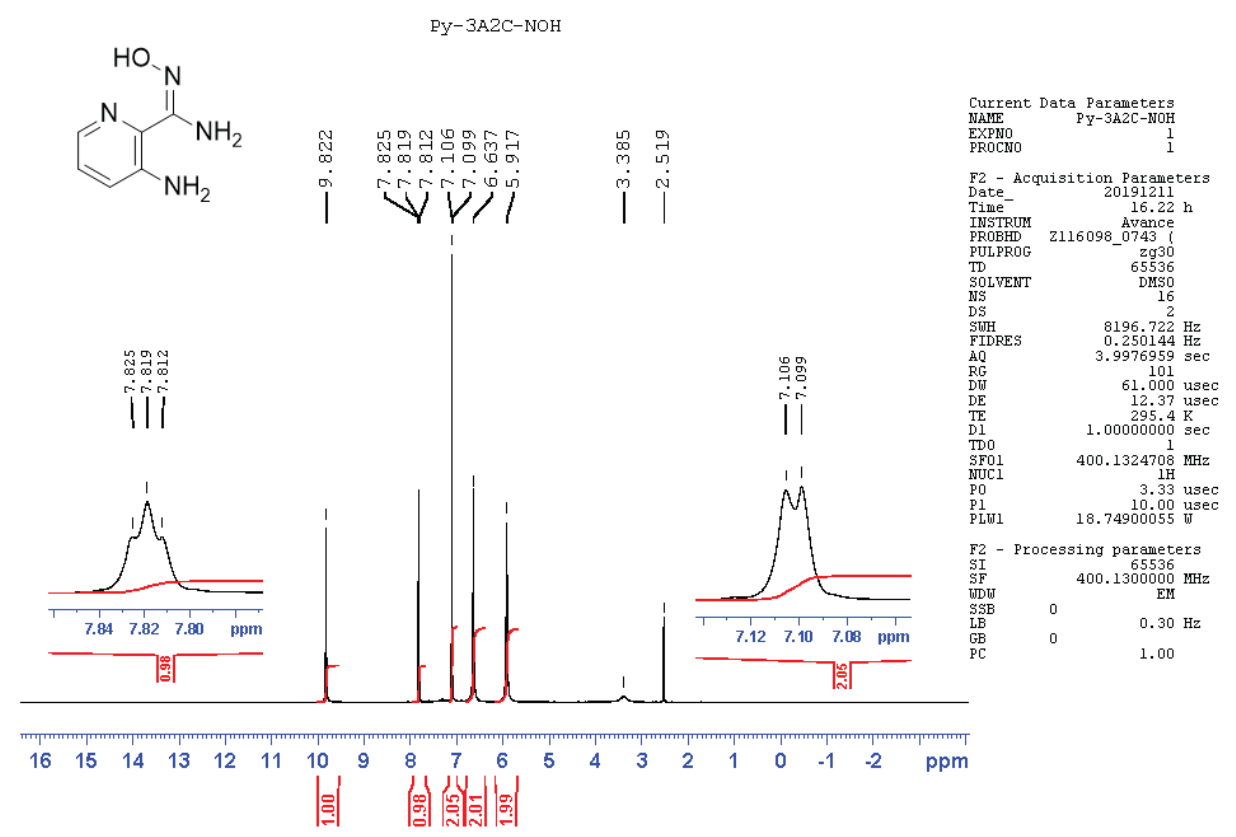

Figure $\mathrm{S} 71 .{ }^{1} \mathrm{H}$ NMR of $4 \mathrm{e}$ in DMSO- $\mathrm{d}_{6}$ operating at $400.13 \mathrm{MHz}$.

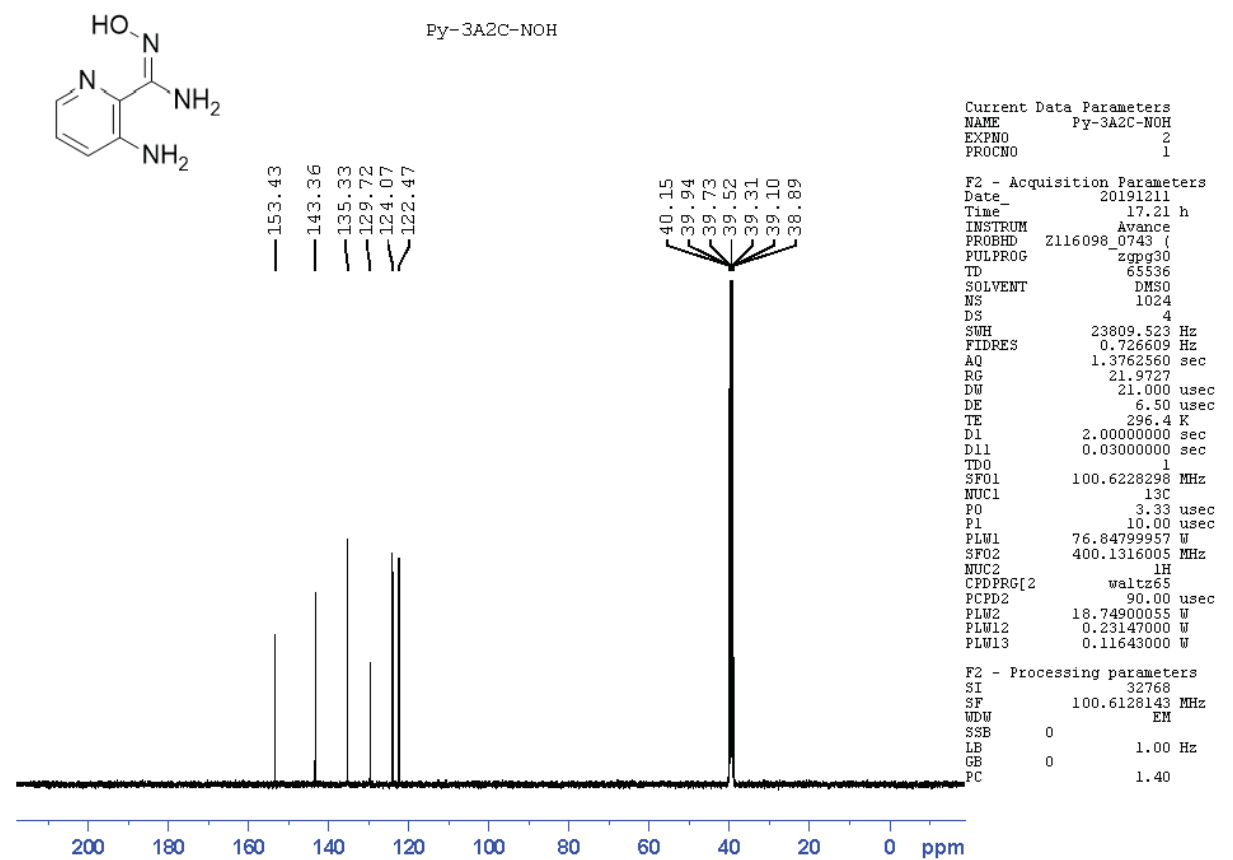

Figure $572 .{ }^{13} \mathrm{C}$ NMR of $4 \mathrm{e}$ in DMSO- $\mathrm{d}_{6}$ operating at $100.61 \mathrm{MHz}$. 


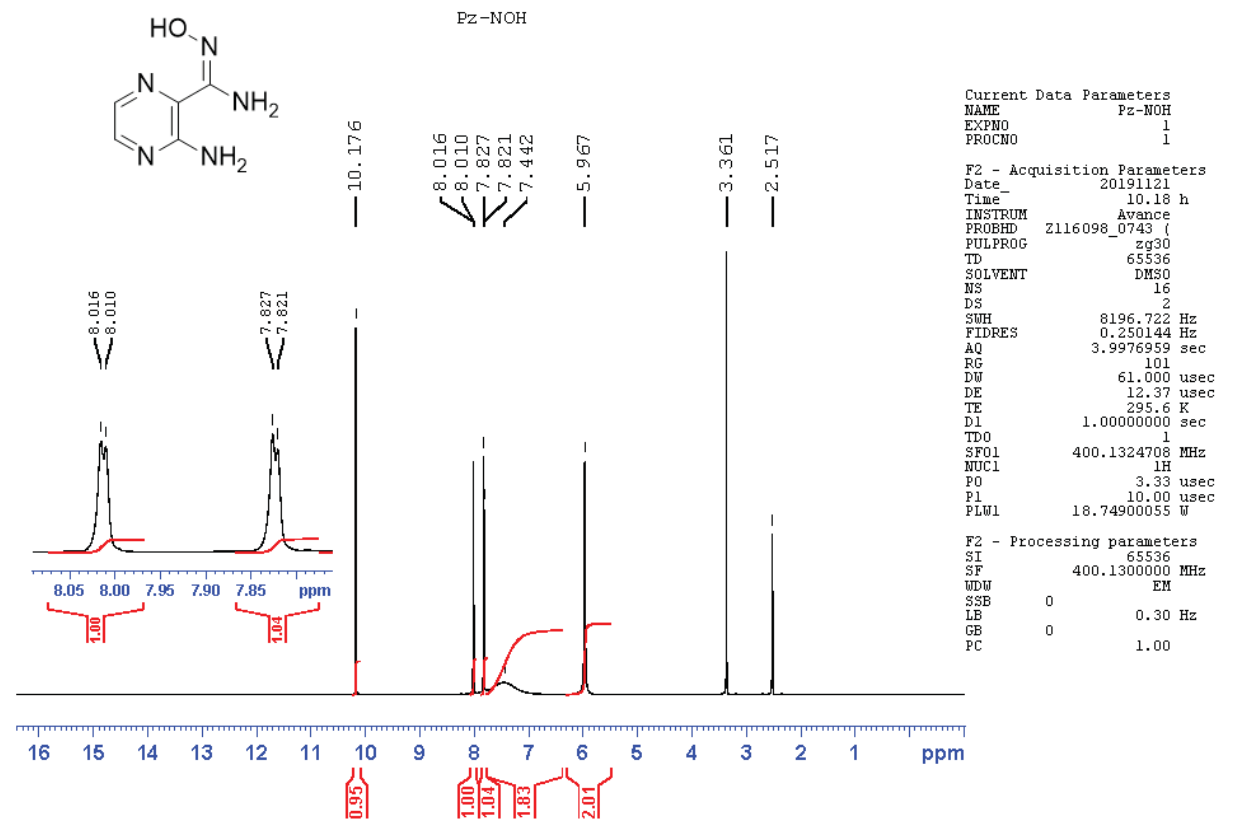

Figure $\mathrm{S} 73 .{ }^{1} \mathrm{H}$ NMR of $\mathbf{4 f}$ in DMSO- $\mathrm{d}_{6}$ operating at $400.13 \mathrm{MHz}$.

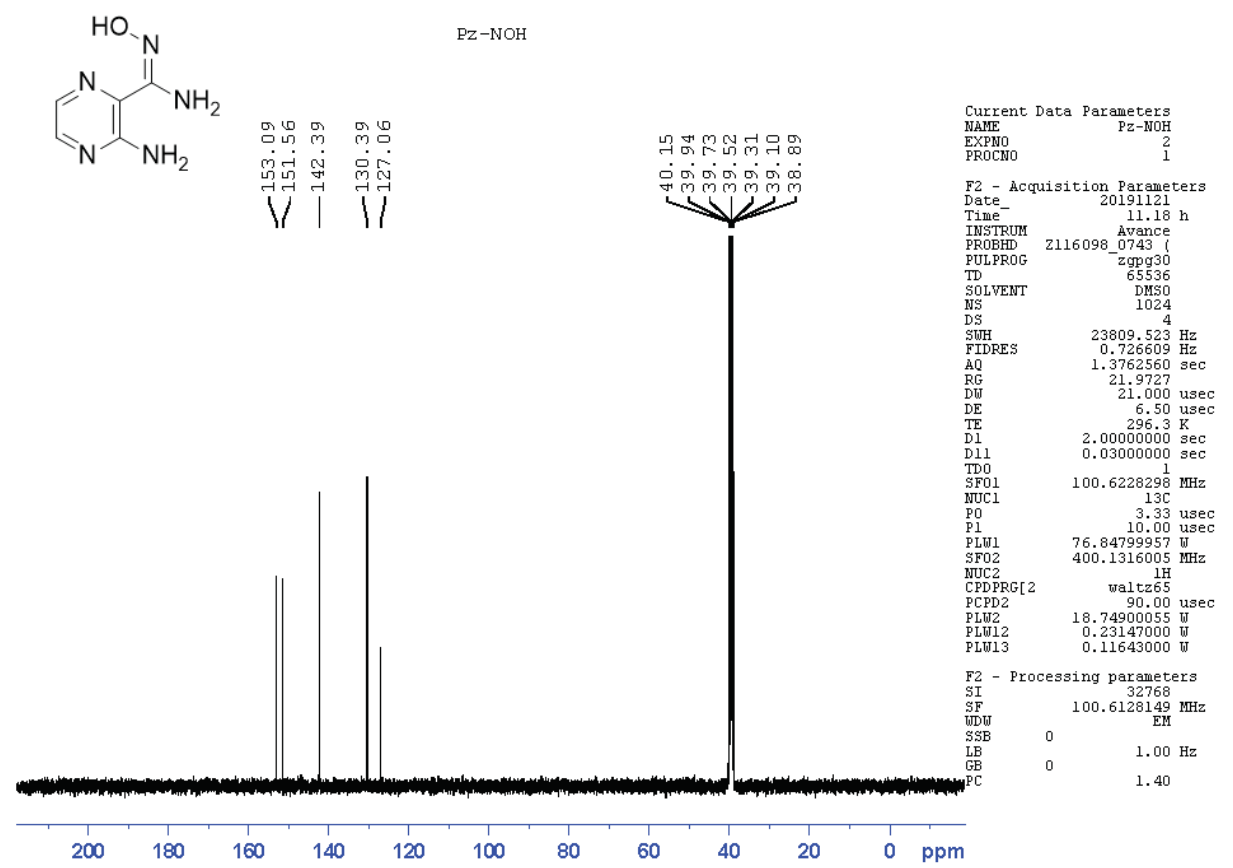

Figure $\mathrm{S} 74 .{ }^{13} \mathrm{C}$ NMR of $\mathbf{4 f}$ in DMSO- $\mathrm{d}_{6}$ operating at $100.61 \mathrm{MHz}$. 


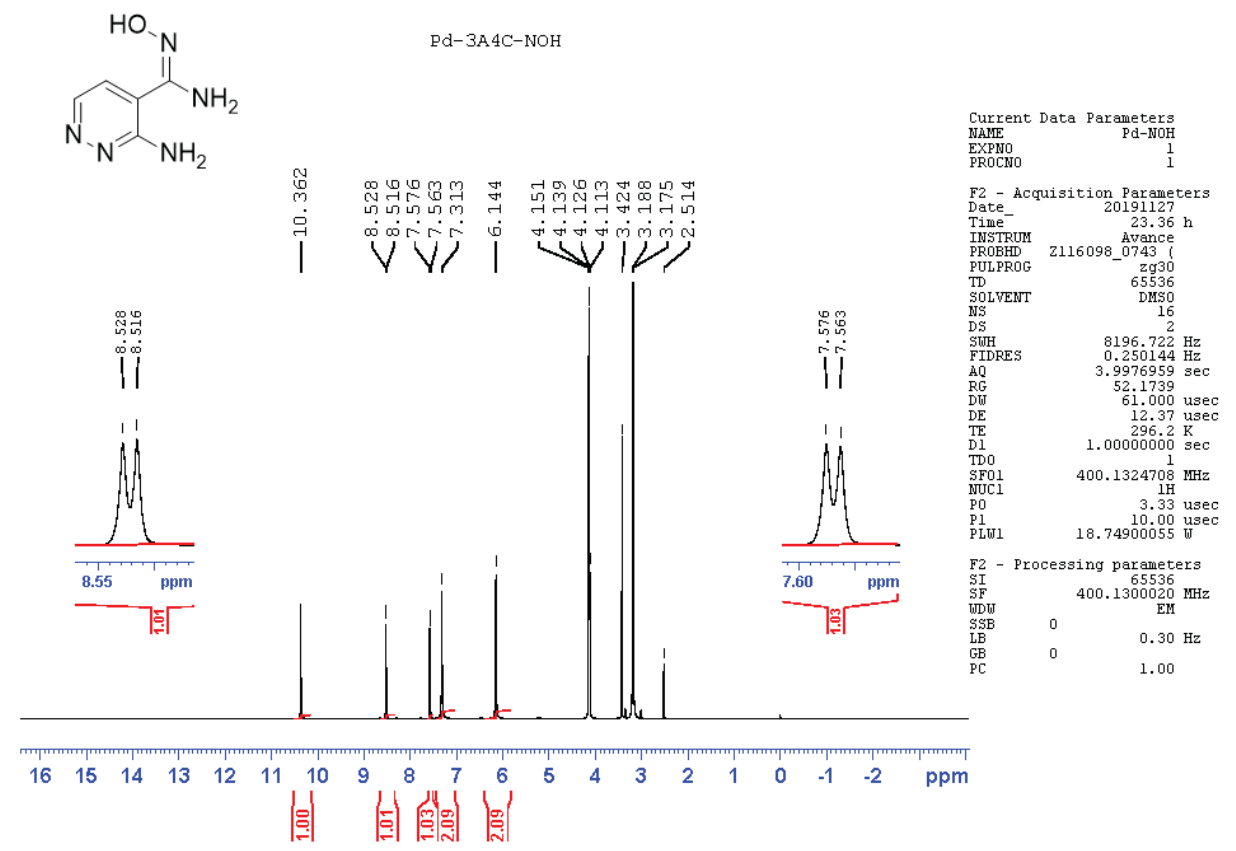

Figure $\mathrm{S} 75 .{ }^{1} \mathrm{H}$ NMR of $\mathbf{4 g}$ in DMSO- $\mathrm{d}_{6}$ operating at $400.13 \mathrm{MHz}$.

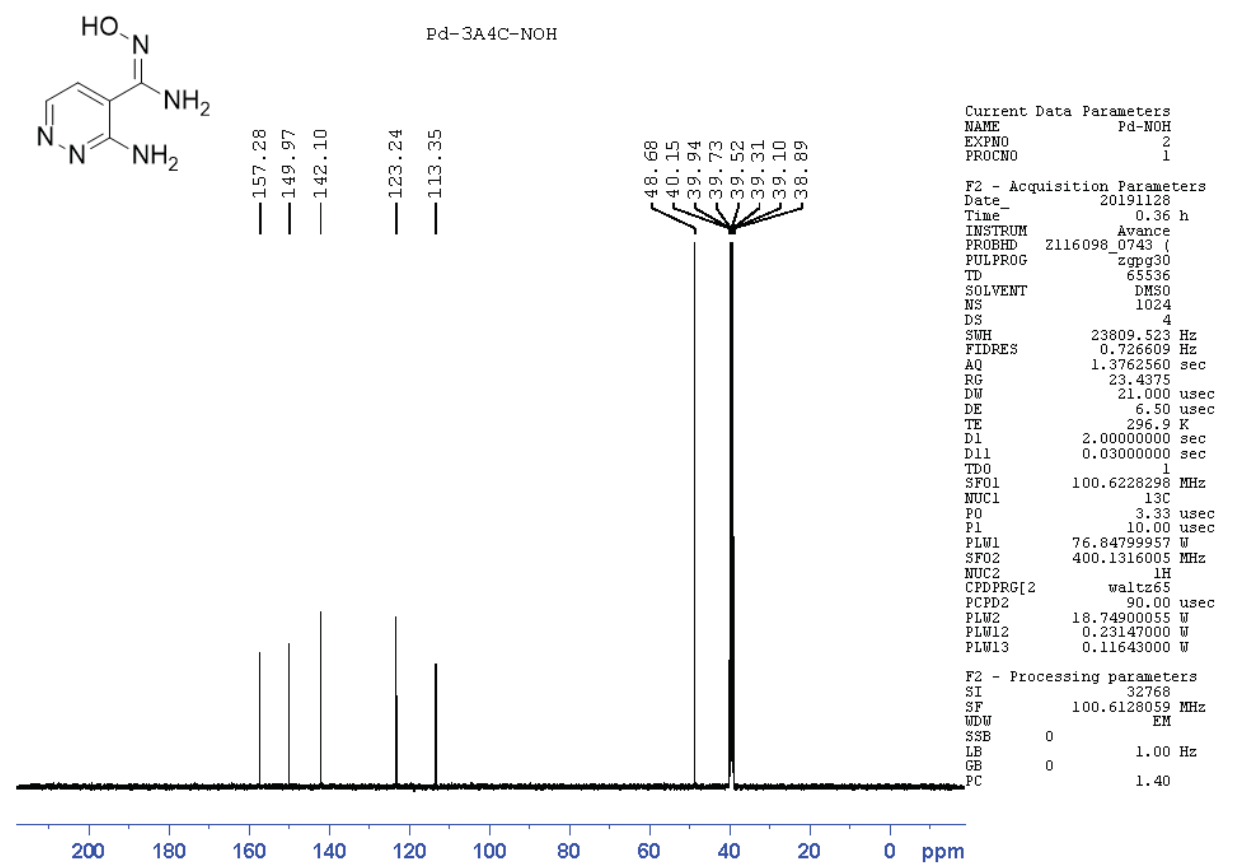

Figure $576 .{ }^{13} \mathrm{C}$ NMR of $4 \mathrm{~g}$ in DMSO-d $\mathrm{d}_{6}$ operating at $100.61 \mathrm{MHz}$. 


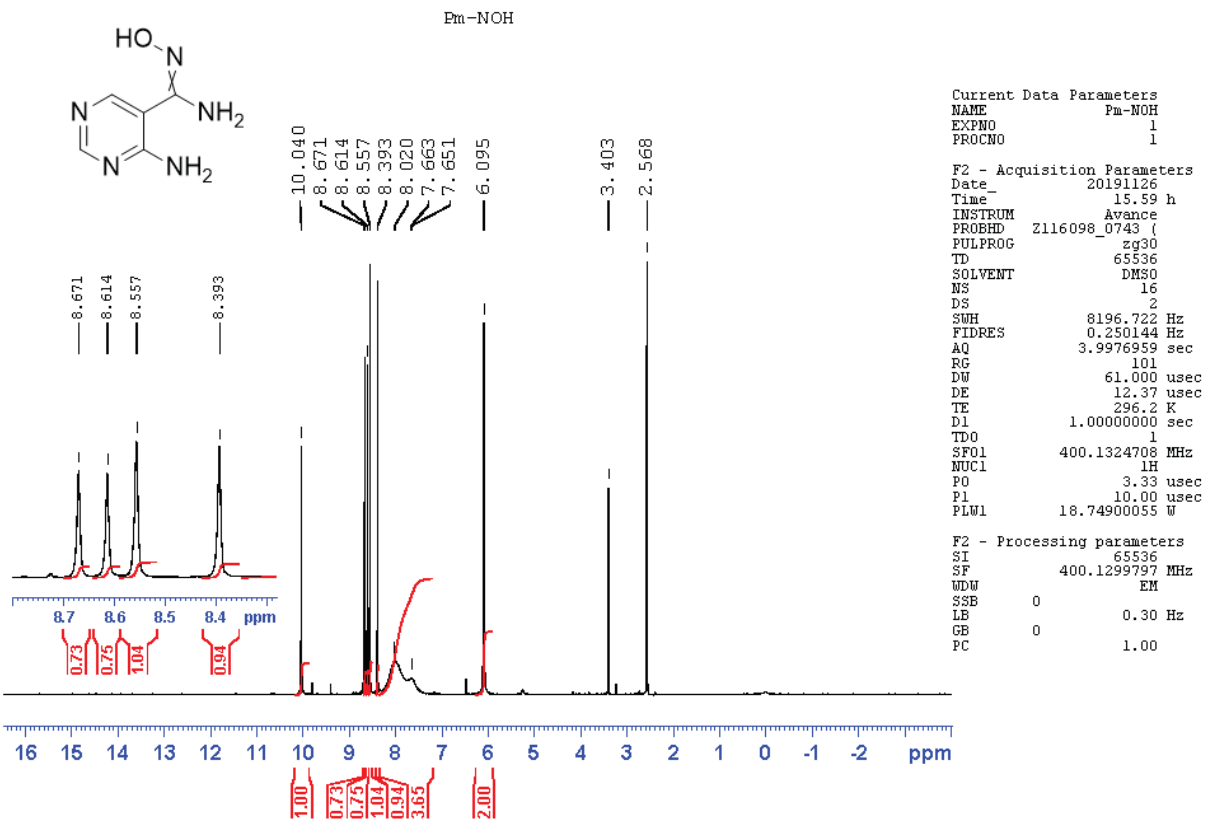

Figure S77. ${ }^{1} \mathrm{H}$ NMR of $\mathbf{4 h - E} / \mathrm{Z}$ in DMSO-d 6 operating at $400.13 \mathrm{MHz}$.

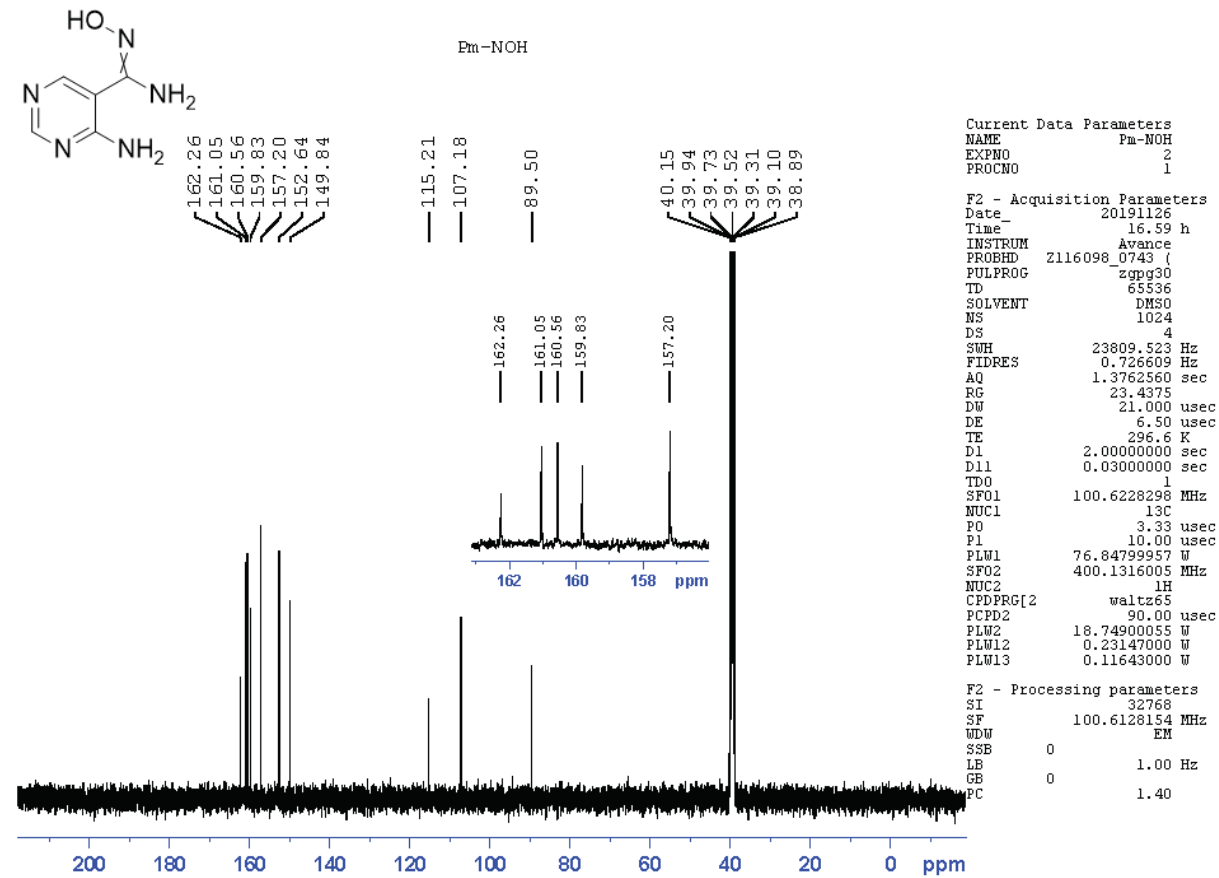

Figure $578 .{ }^{13} \mathrm{C}$ NMR of $\mathbf{4 h}-\mathrm{E} / \mathrm{Z}$ in DMSO- $\mathrm{d}_{6}$ operating at $100.61 \mathrm{MHz}$. 


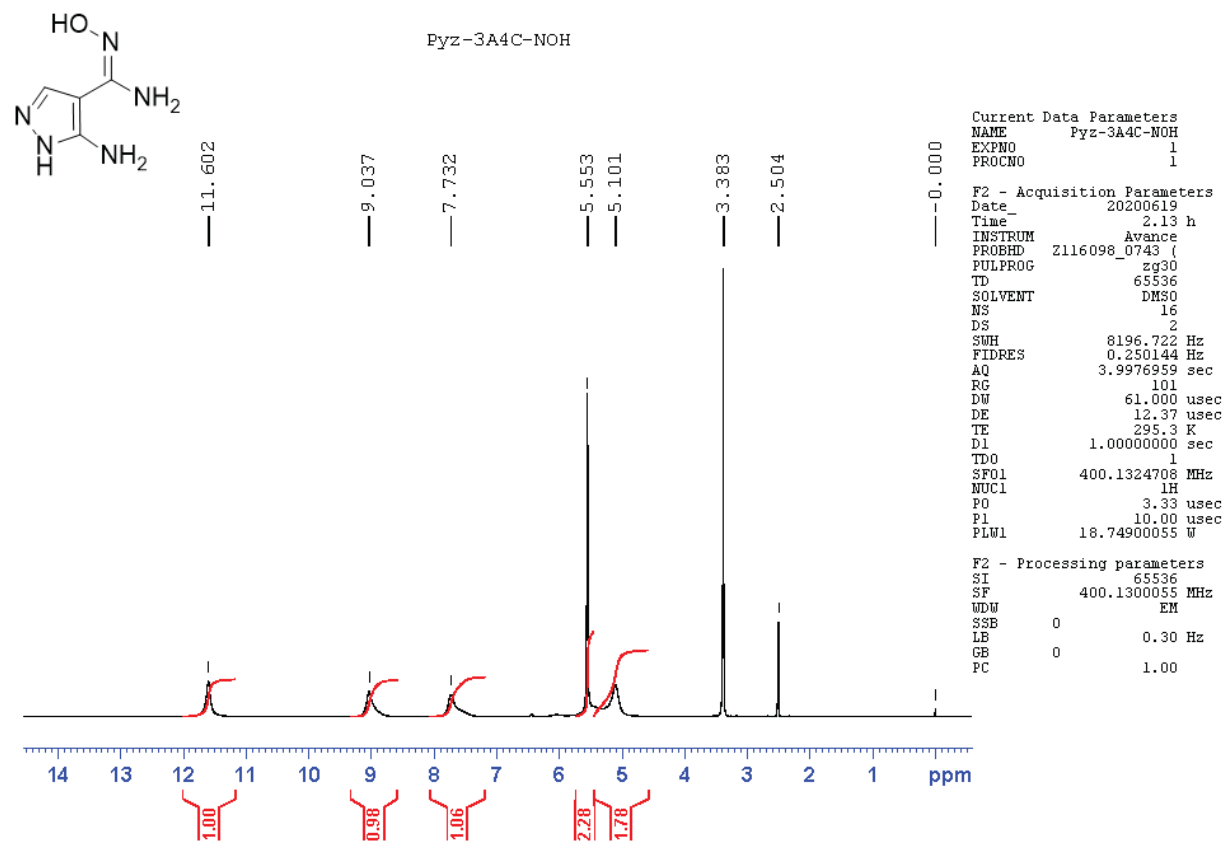

Figure S79. ${ }^{1} \mathrm{H}$ NMR of $4 \mathbf{i}$ in DMSO- $\mathrm{d}_{6}$ operating at $400.13 \mathrm{MHz}$.

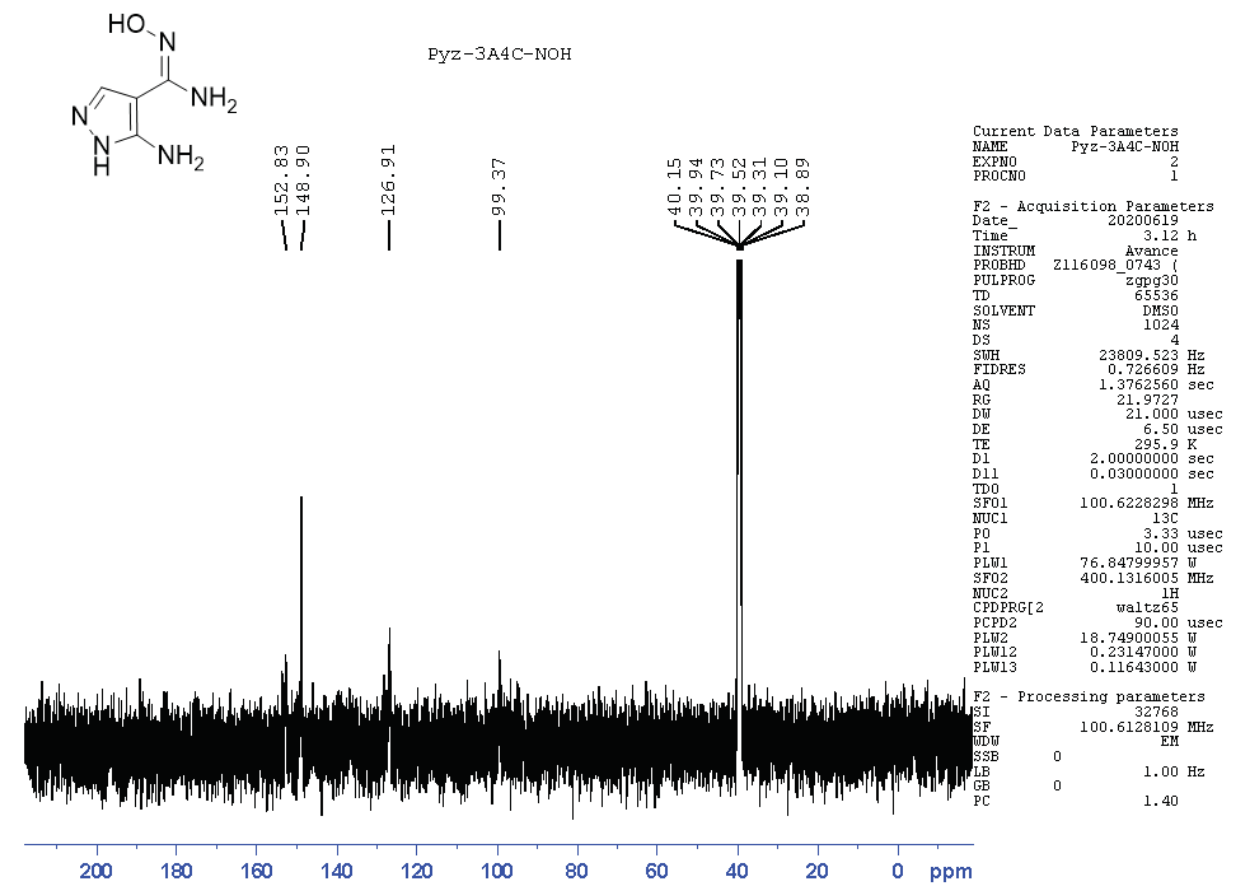

Figure S80. ${ }^{13} \mathrm{C}$ NMR of $4 \mathbf{i}$ in DMSO- $\mathrm{d}_{6}$ operating at $100.61 \mathrm{MHz}$. 


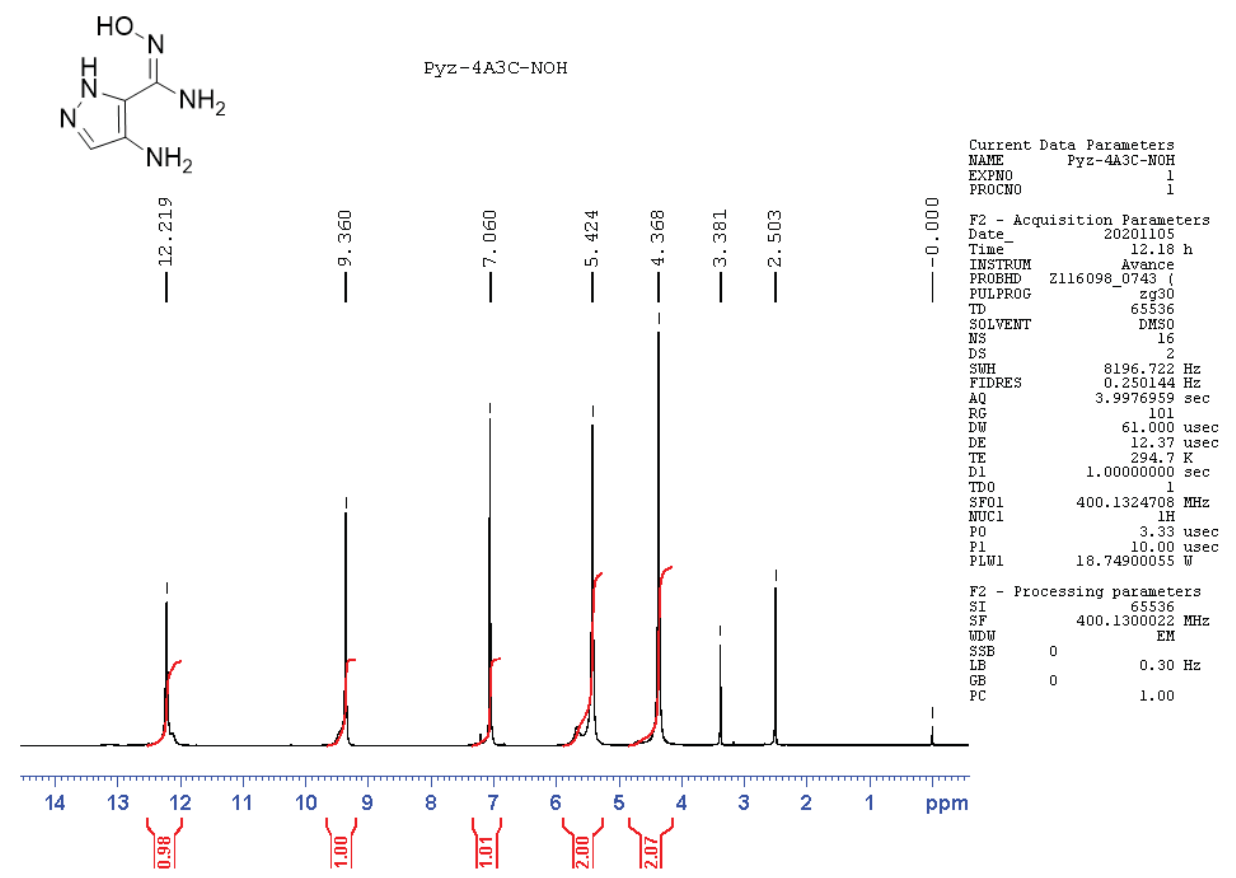

Figure $\mathrm{S} 81 .{ }^{1} \mathrm{H}$ NMR of $4 \mathbf{j}$ in DMSO- $\mathrm{d}_{6}$ operating at $400.13 \mathrm{MHz}$.

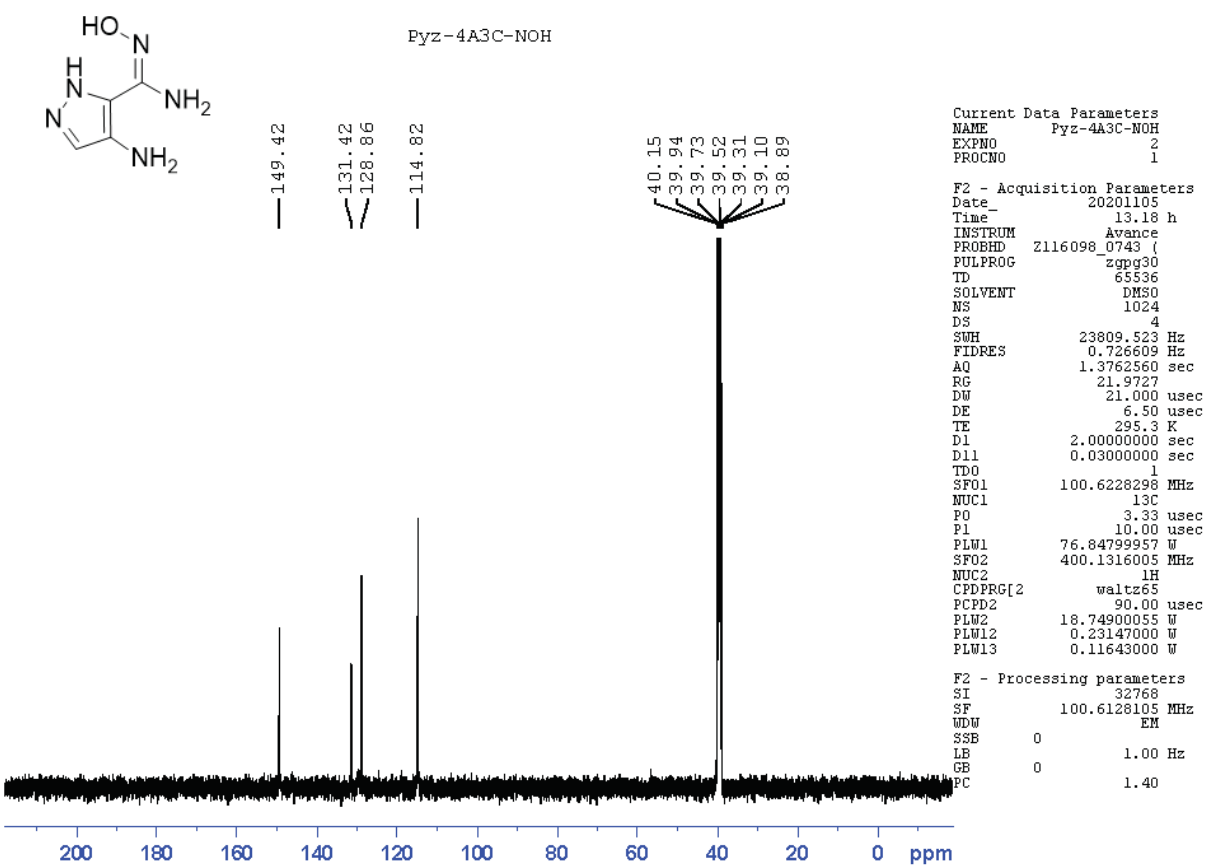

Figure $582 .{ }^{13} \mathrm{C}$ NMR of $\mathbf{4} \mathbf{j}$ in DMSO- $\mathrm{d}_{6}$ operating at $100.61 \mathrm{MHz}$. 


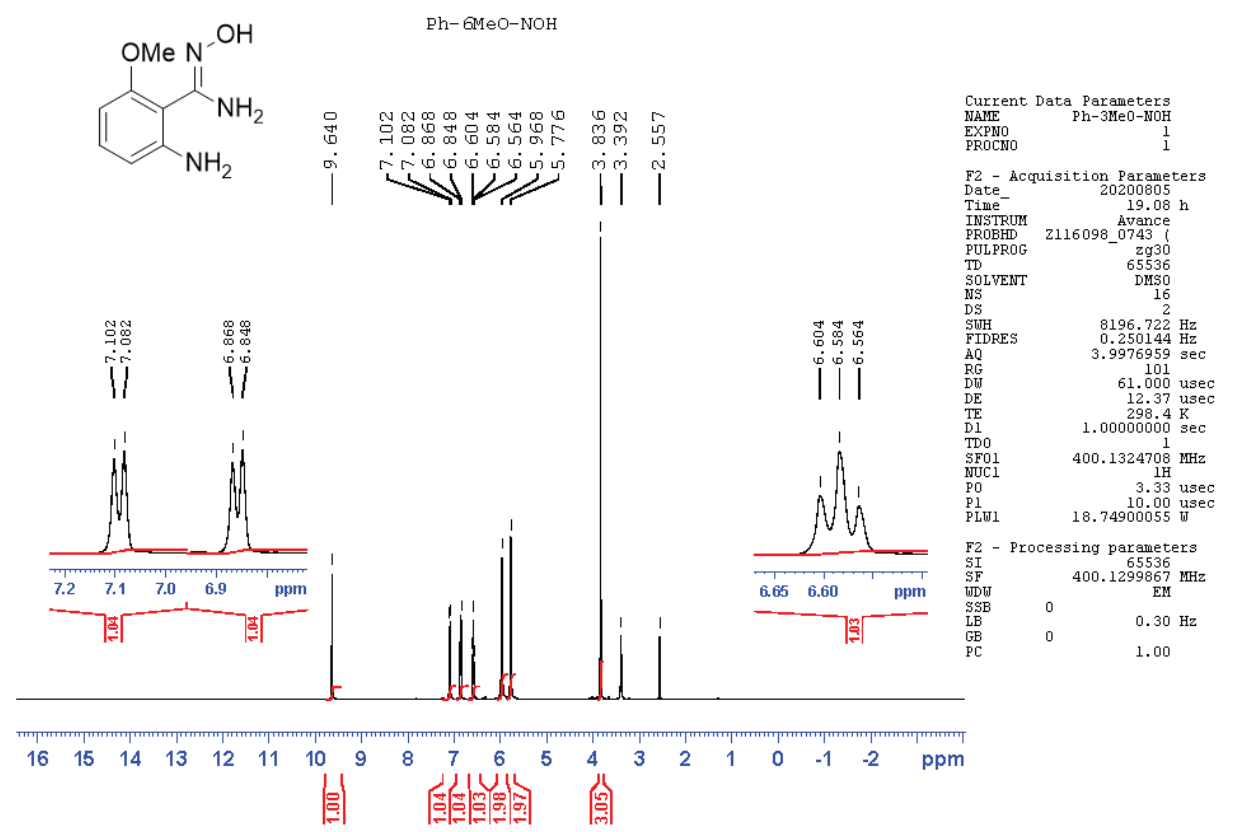

Figure S83. ${ }^{1} \mathrm{H}$ NMR of $\mathbf{4 k}$ in DMSO-d 6 operating at $400.13 \mathrm{MHz}$.

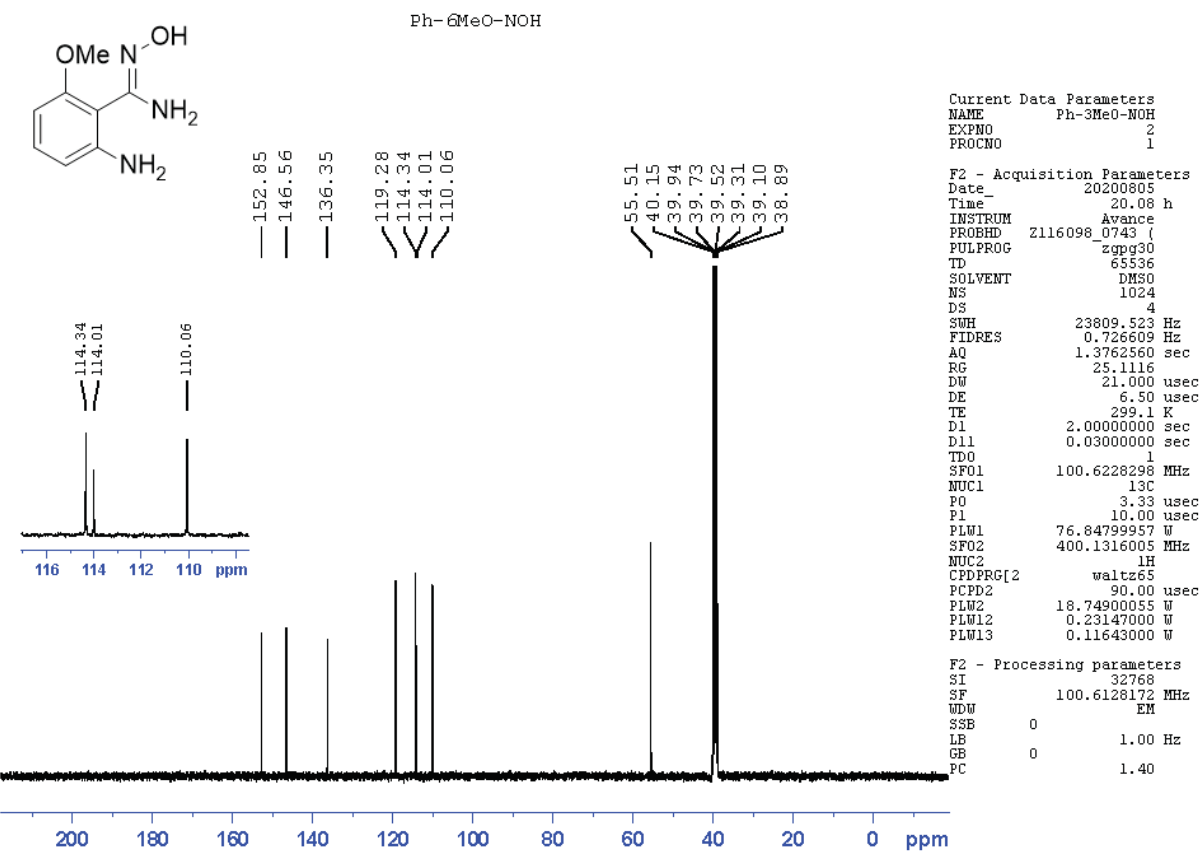

Figure $584 .{ }^{13} \mathrm{C}$ NMR of $\mathbf{4 k}$ in DMSO-d $\mathrm{d}_{6}$ operating at $100.61 \mathrm{MHz}$. 


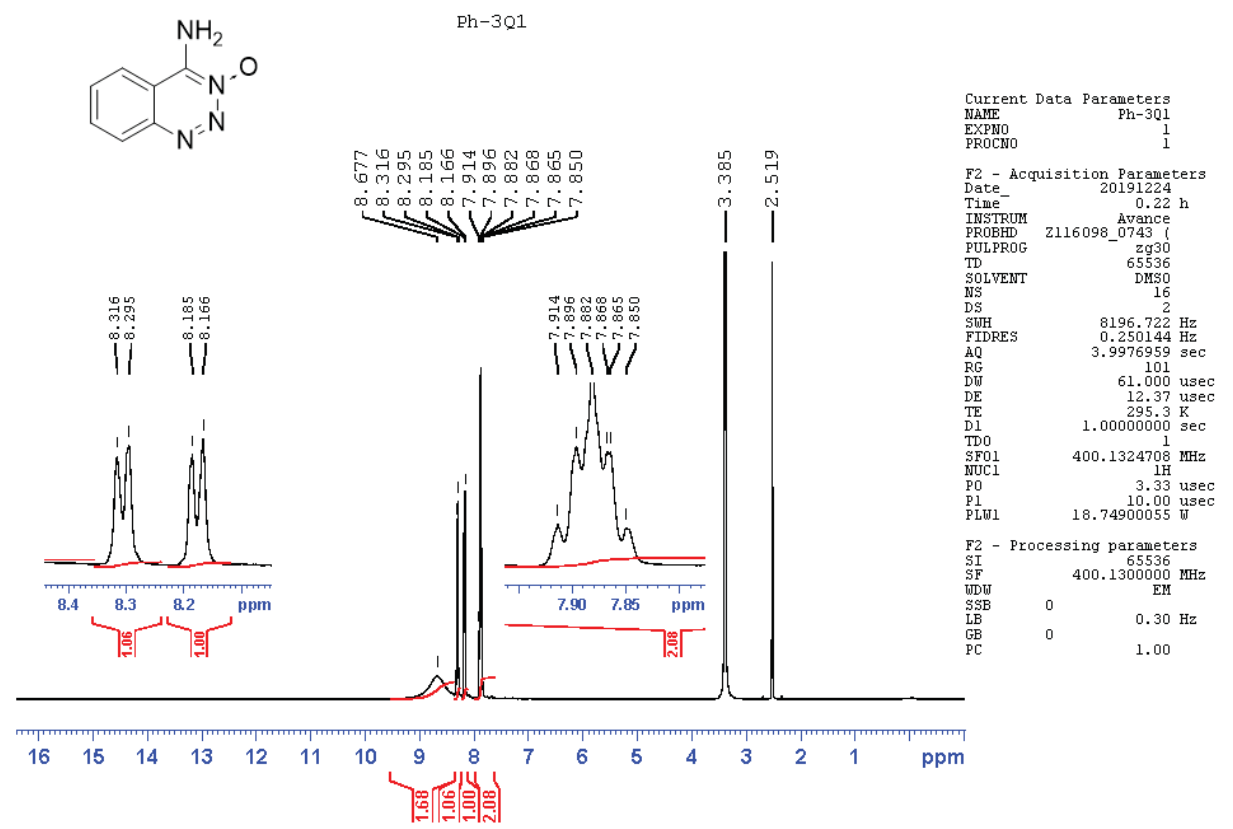

Figure $\mathrm{S} 85 .{ }^{1} \mathrm{H}$ NMR of $5 \mathrm{a}$ in DMSO- $\mathrm{d}_{6}$ operating at $400.13 \mathrm{MHz}$.

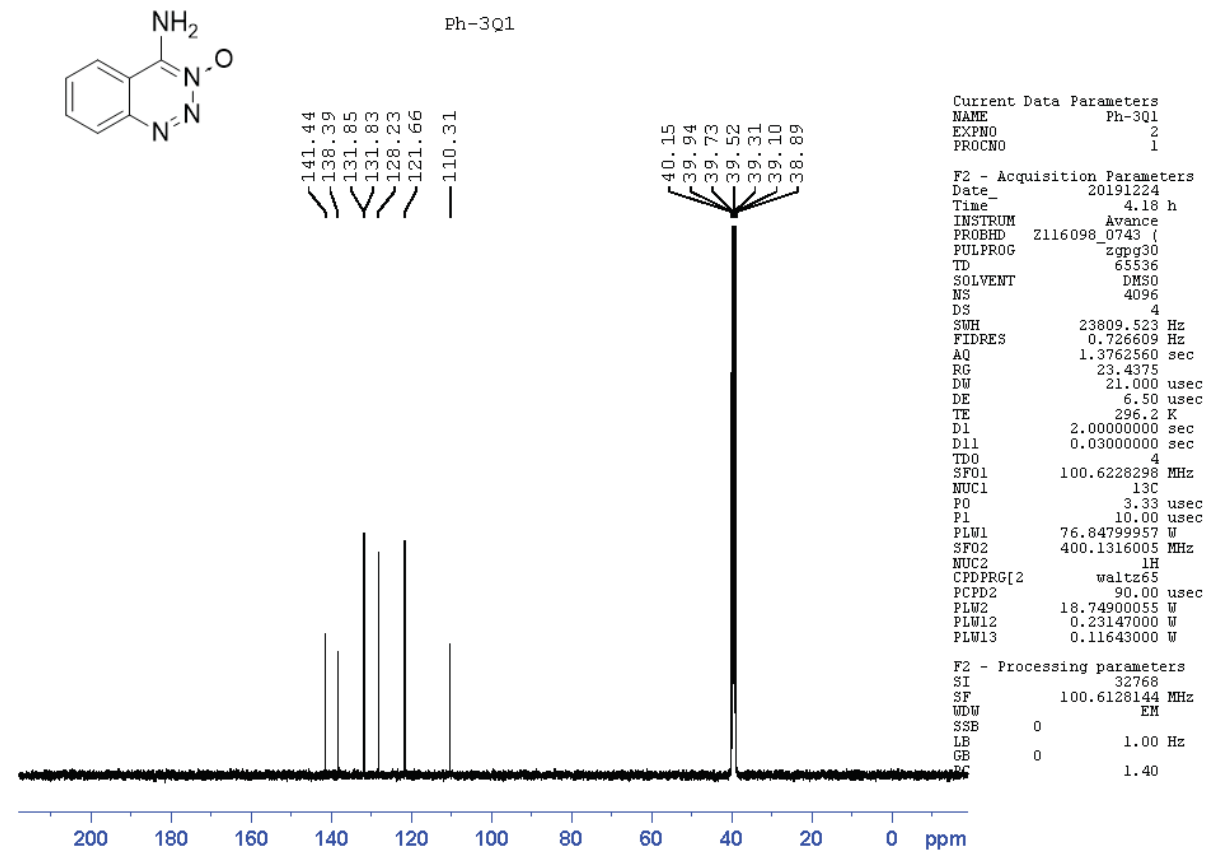

Figure $586 .{ }^{13} \mathrm{C}$ NMR of 5 a in DMSO- $\mathrm{d}_{6}$ operating at $100.61 \mathrm{MHz}$. 


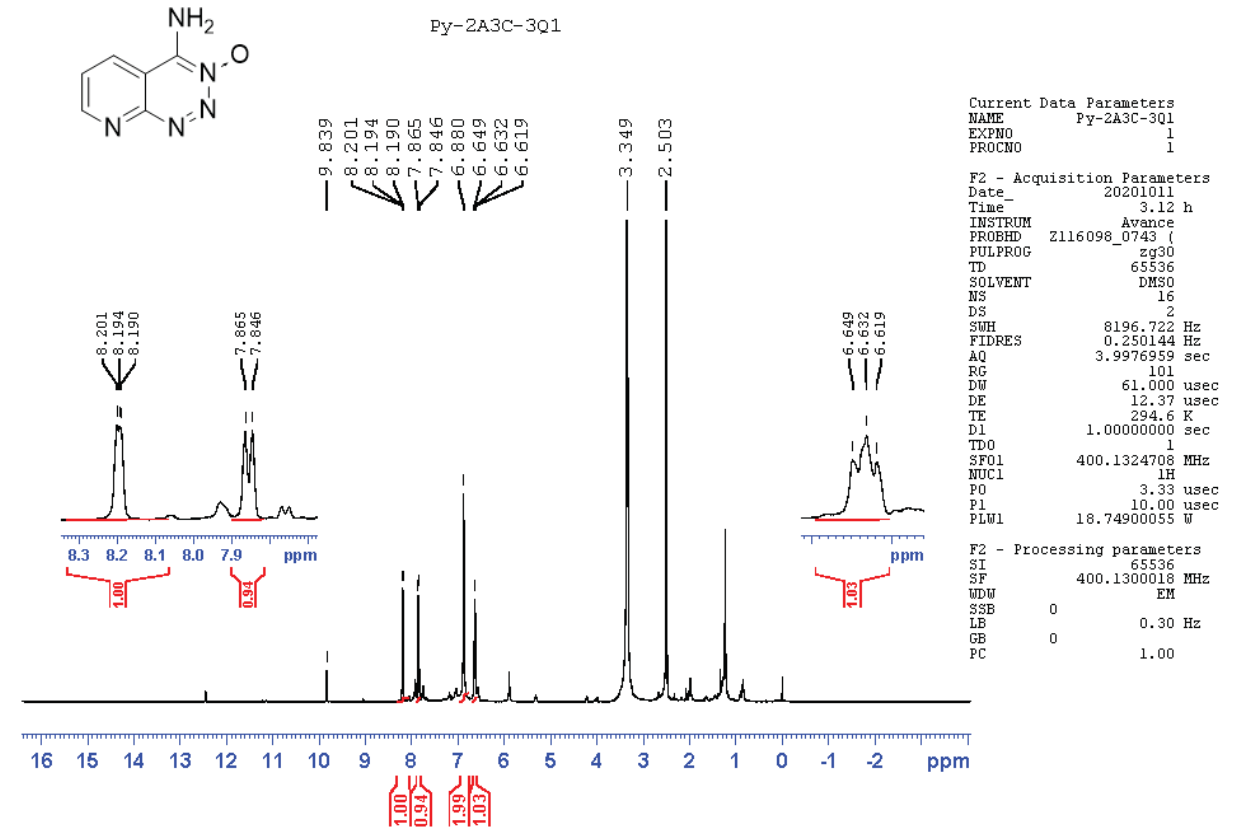

Figure S87. ${ }^{1} \mathrm{H}$ NMR of $\mathbf{5 b}$ in DMSO-d $\mathrm{d}_{6}$ operating at $400.13 \mathrm{MHz}$.

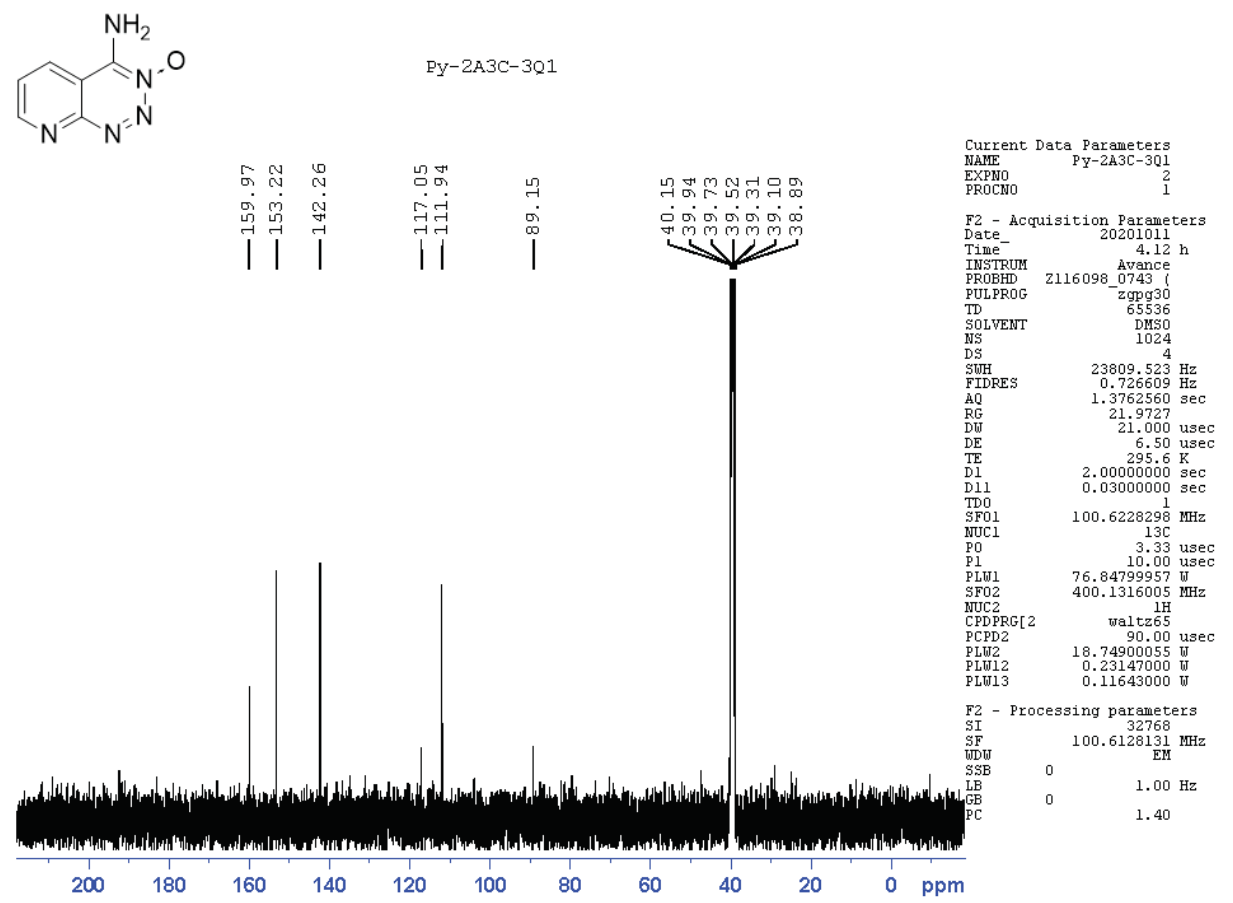

Figure $588 .{ }^{13} \mathrm{C}$ NMR of $\mathbf{5 b}$ in DMSO-d 6 operating at $100.61 \mathrm{MHz}$. 


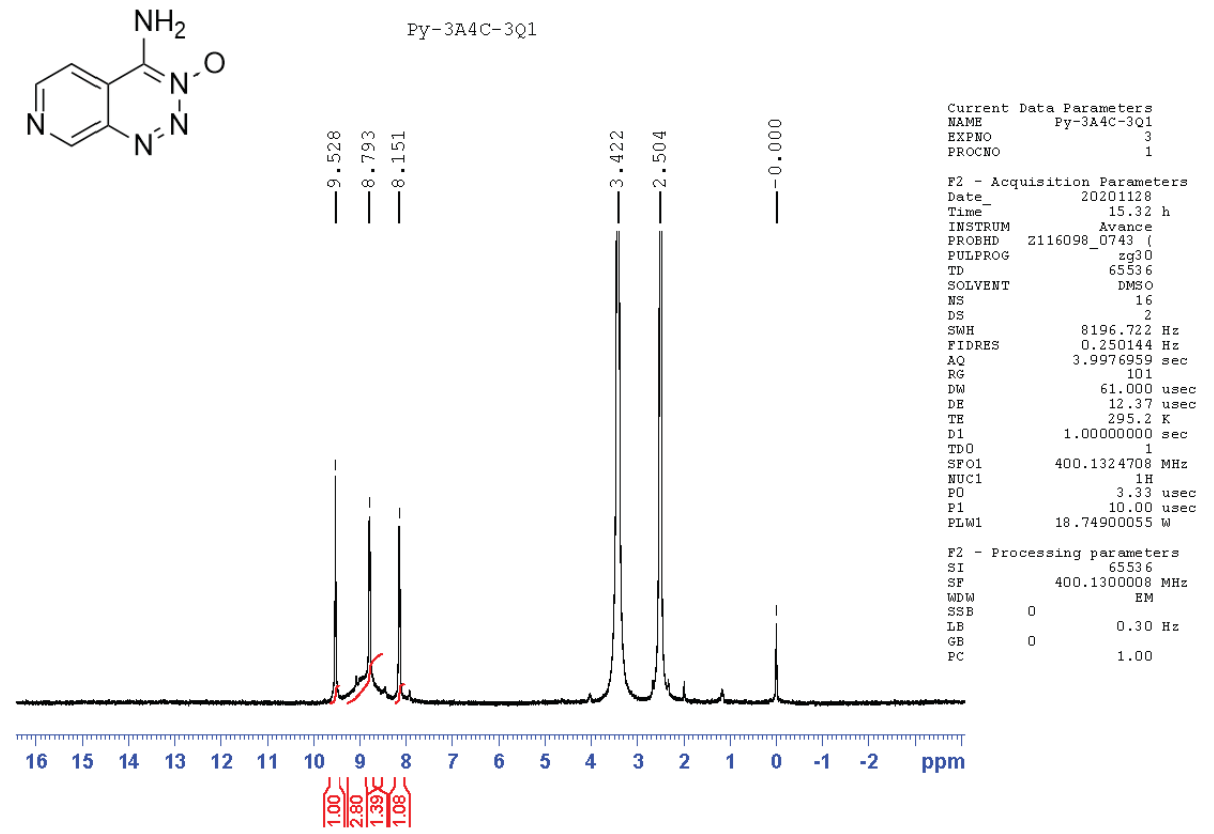

Figure S89. ${ }^{1} \mathrm{H}$ NMR of $5 c$ in DMSO- $\mathrm{d}_{6}$ operating at $400.13 \mathrm{MHz}$.

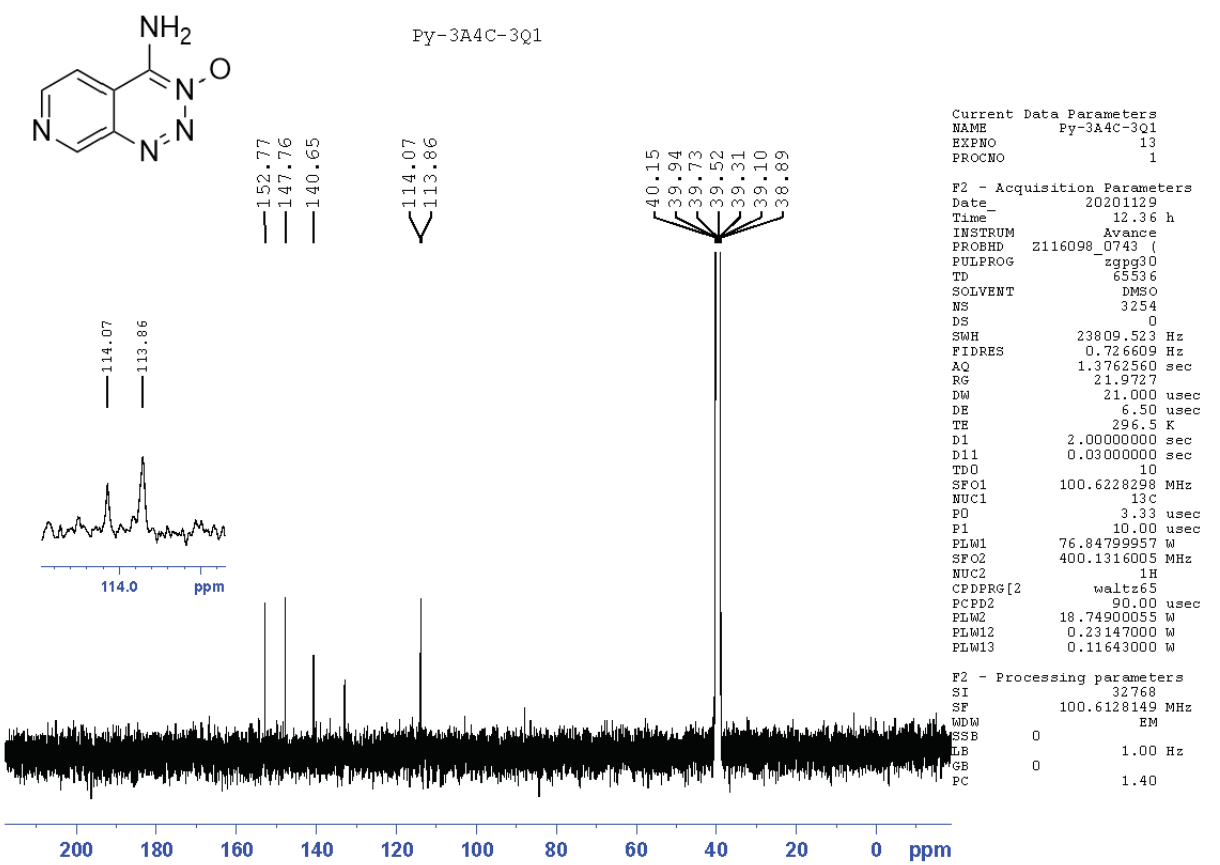

Figure S90. ${ }^{13} \mathrm{C}$ NMR of $5 \mathrm{c}$ in DMSO- $\mathrm{d}_{6}$ operating at $100.61 \mathrm{MHz}$. 


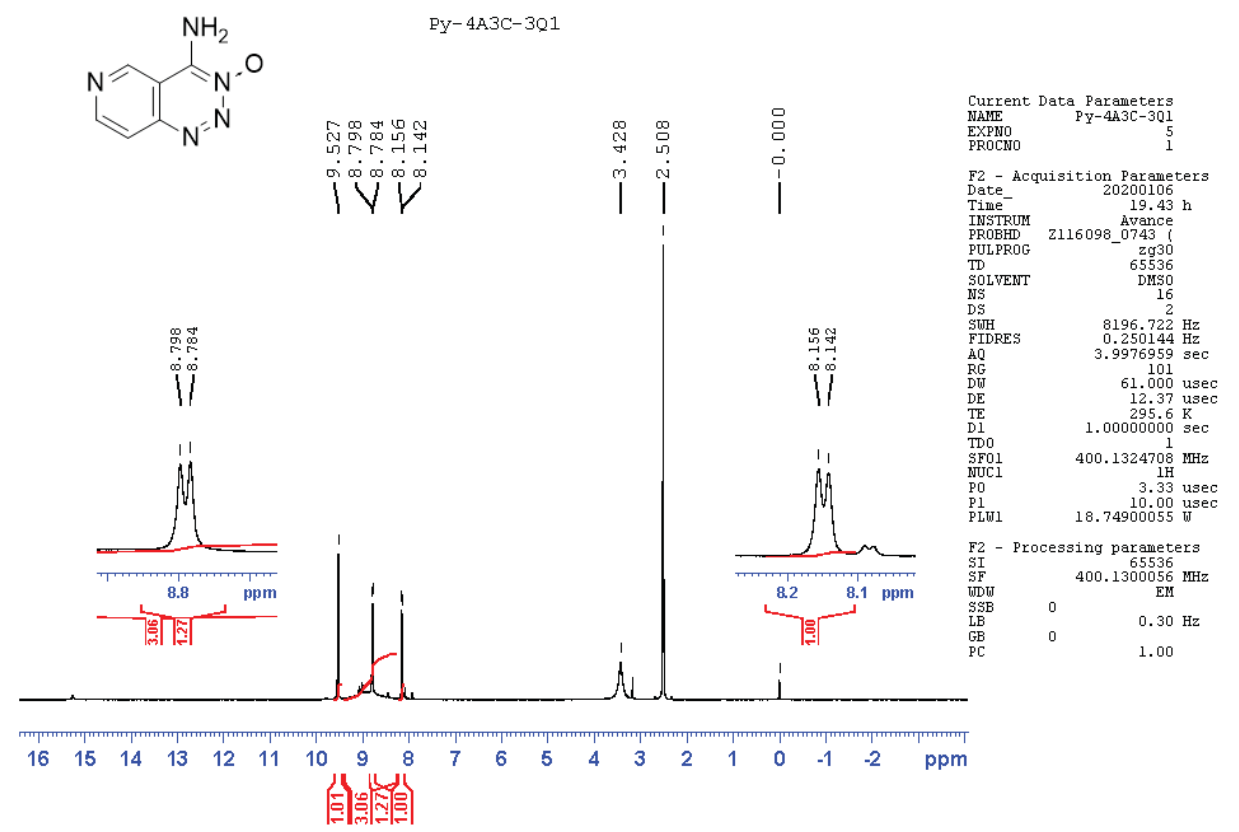

Figure S91. ${ }^{1} \mathrm{H}$ NMR of $\mathbf{5 d}$ in DMSO- $\mathrm{d}_{6}$ operating at $400.13 \mathrm{MHz}$.

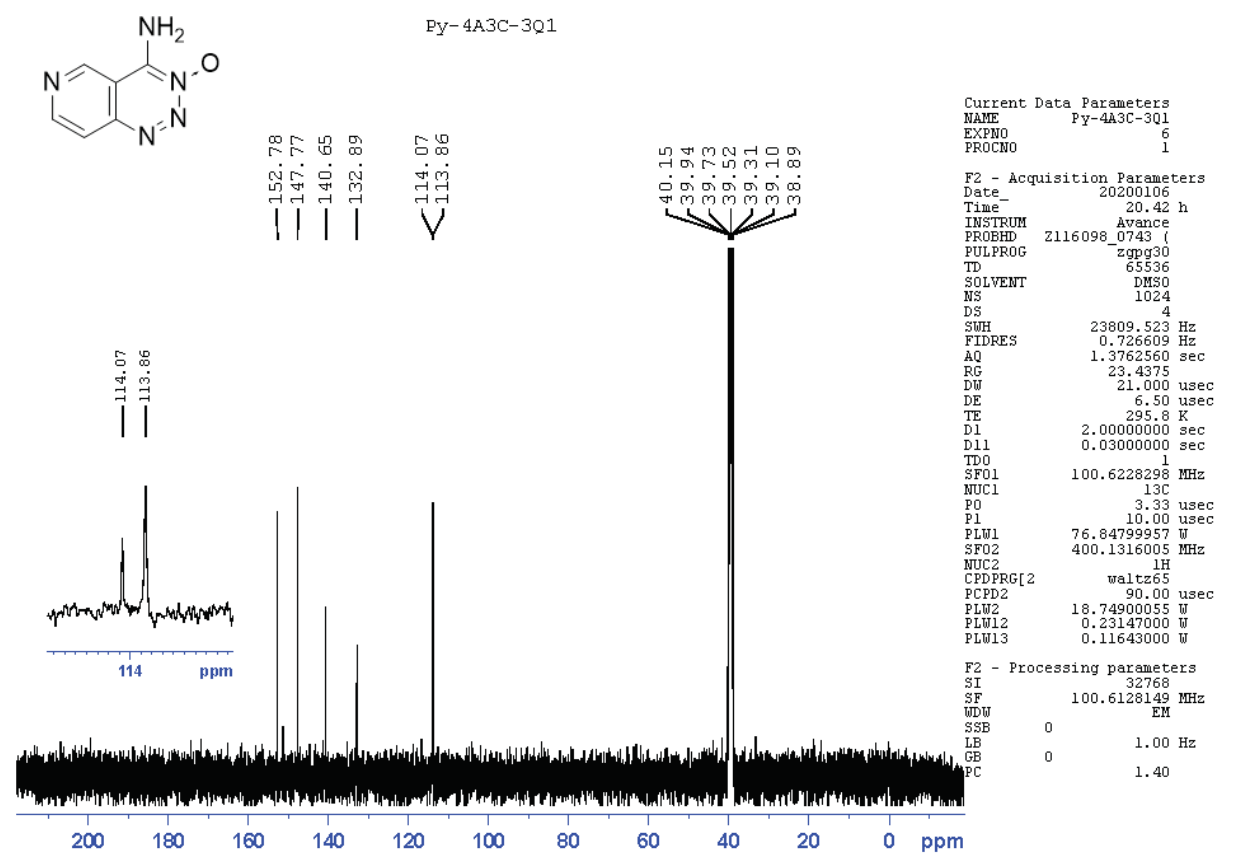

Figure S92. ${ }^{13} \mathrm{C}$ NMR of $\mathbf{5 d}$ in DMSO-d 6 operating at $100.61 \mathrm{MHz}$. 


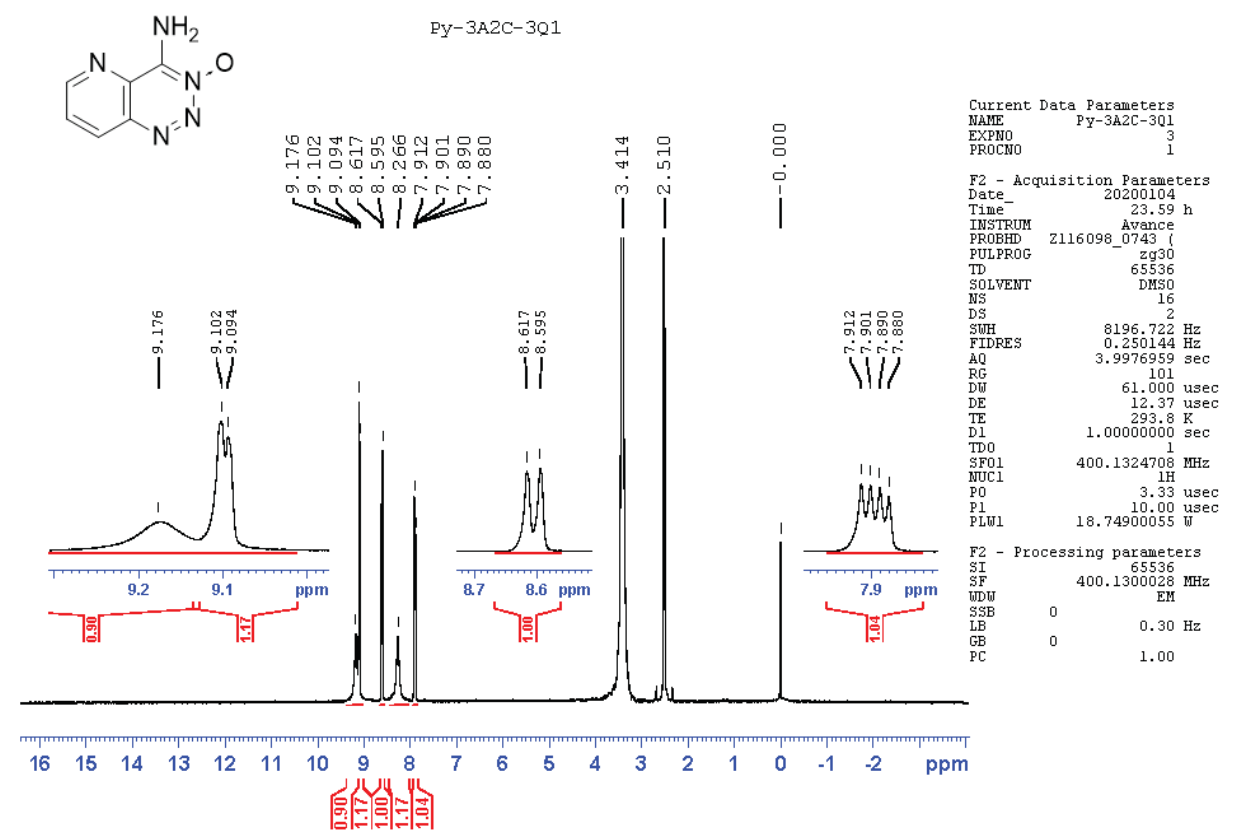

Figure S93. ${ }^{1} \mathrm{H}$ NMR of $5 e$ in DMSO-d $\mathrm{d}_{6}$ operating at $400.13 \mathrm{MHz}$.

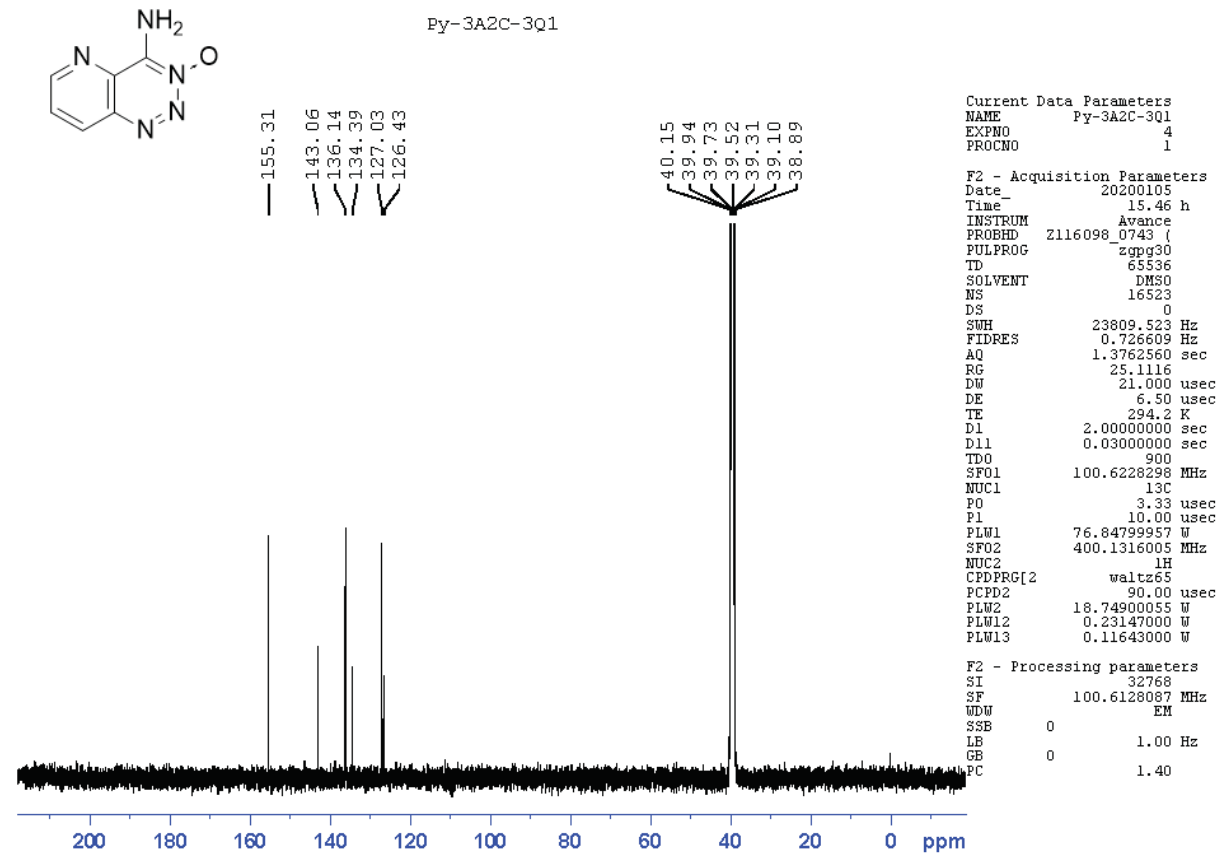

Figure $594 .{ }^{13} \mathrm{C}$ NMR of $5 e$ in DMSO-d 6 operating at $100.61 \mathrm{MHz}$. 


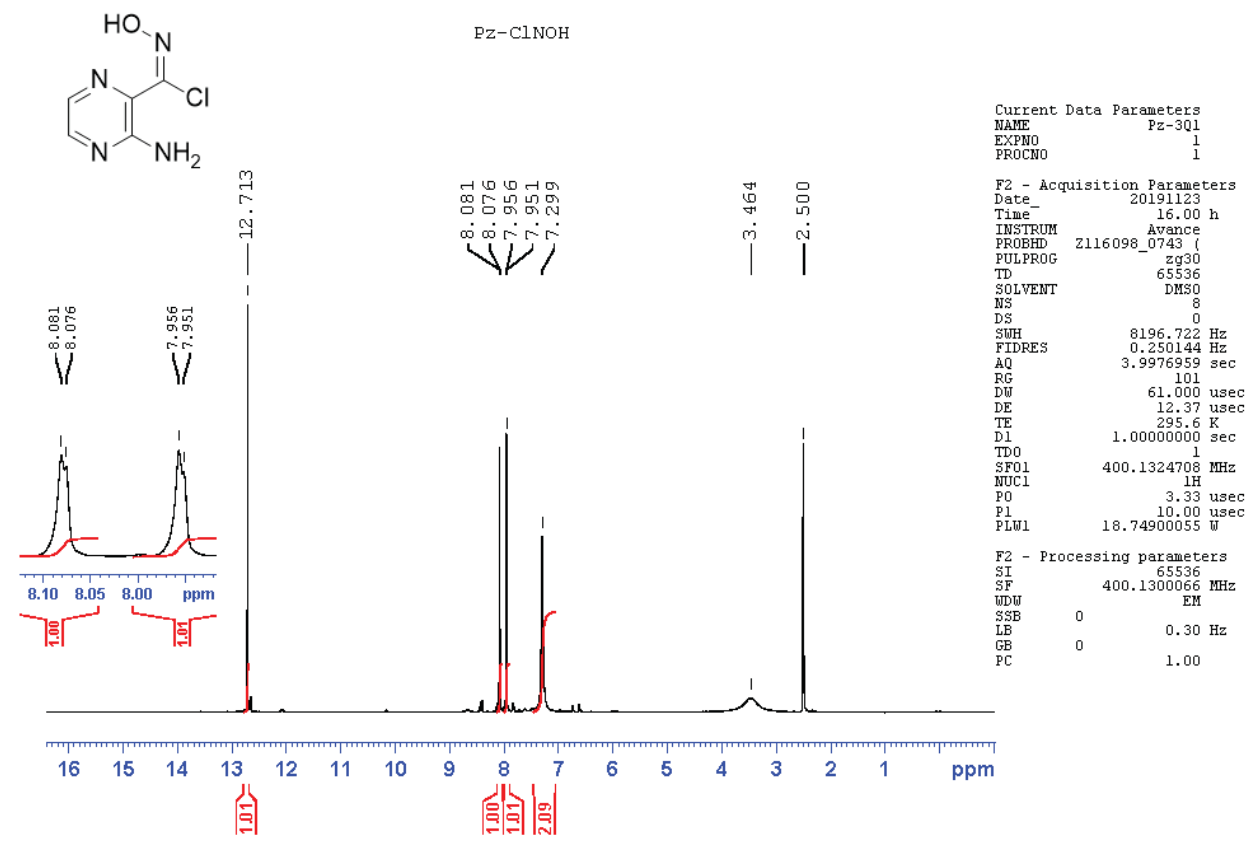

Figure S95. ${ }^{1} \mathrm{H}$ NMR of $\mathbf{5 f - 1}$ in DMSO- $\mathrm{d}_{6}$ operating at $400.13 \mathrm{MHz}$.

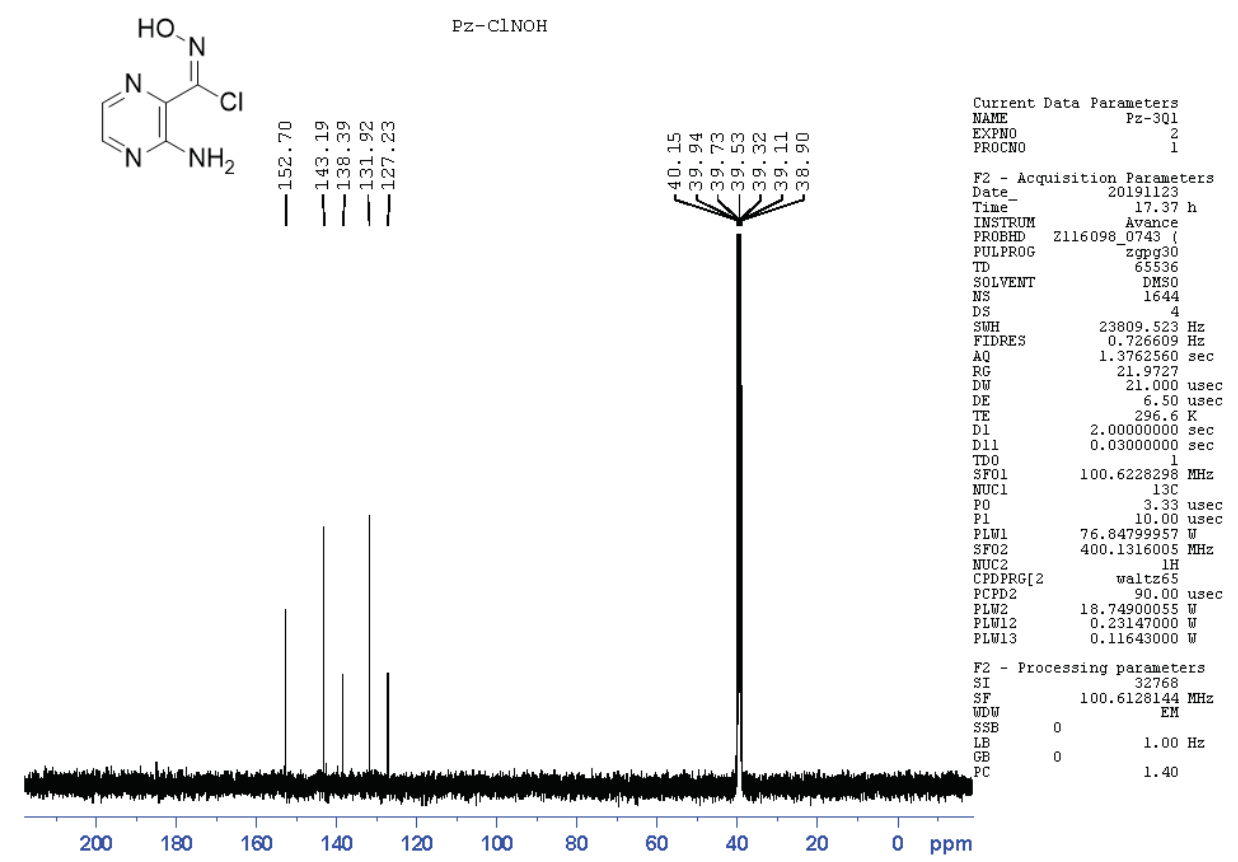

Figure S96. ${ }^{13} \mathrm{C}$ NMR of $\mathbf{5 f - 1}$ in DMSO-d $\mathrm{d}_{6}$ operating at $100.61 \mathrm{MHz}$. 


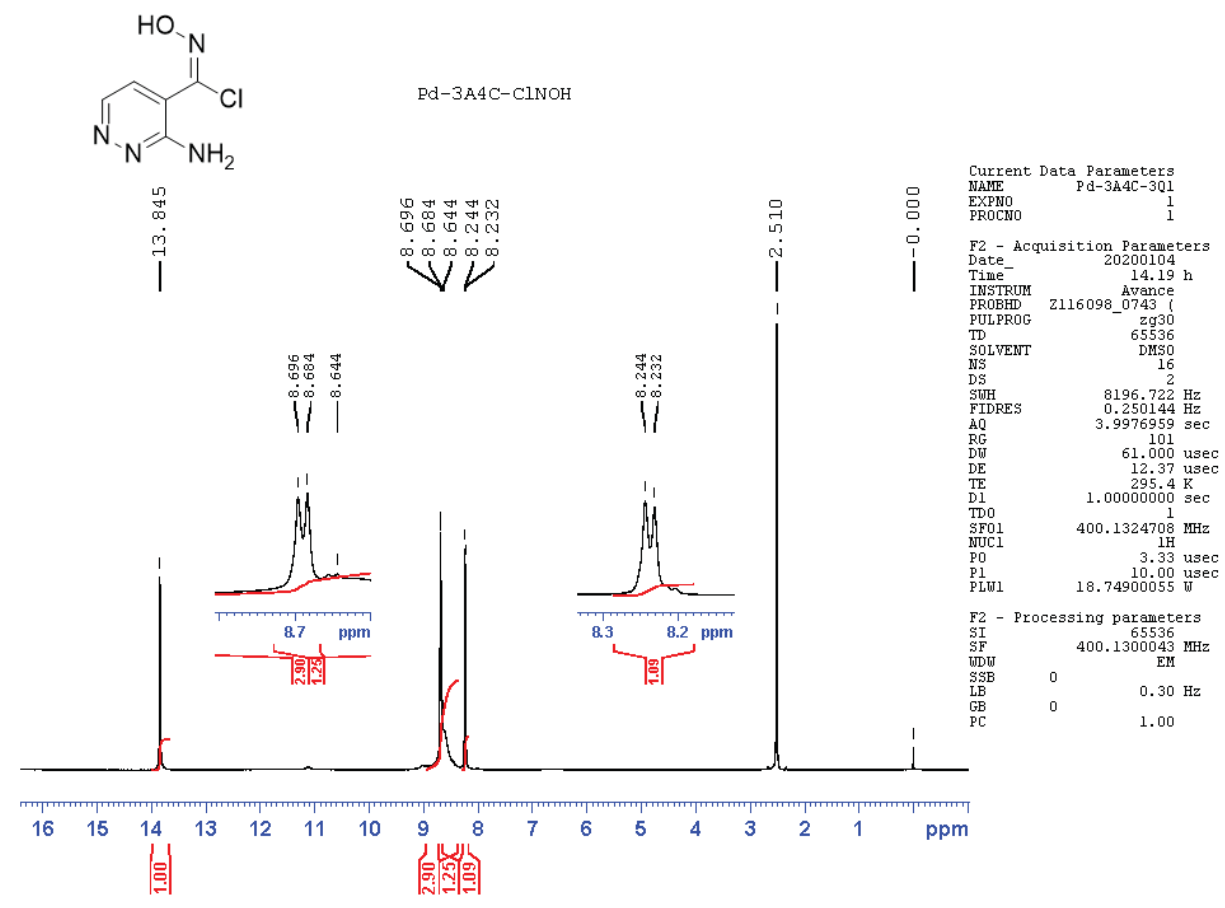

Figure S97. ${ }^{1} \mathrm{H}$ NMR of $5 \mathrm{~g}-1$ in DMSO- $\mathrm{d}_{6}$ operating at $400.13 \mathrm{MHz}$.

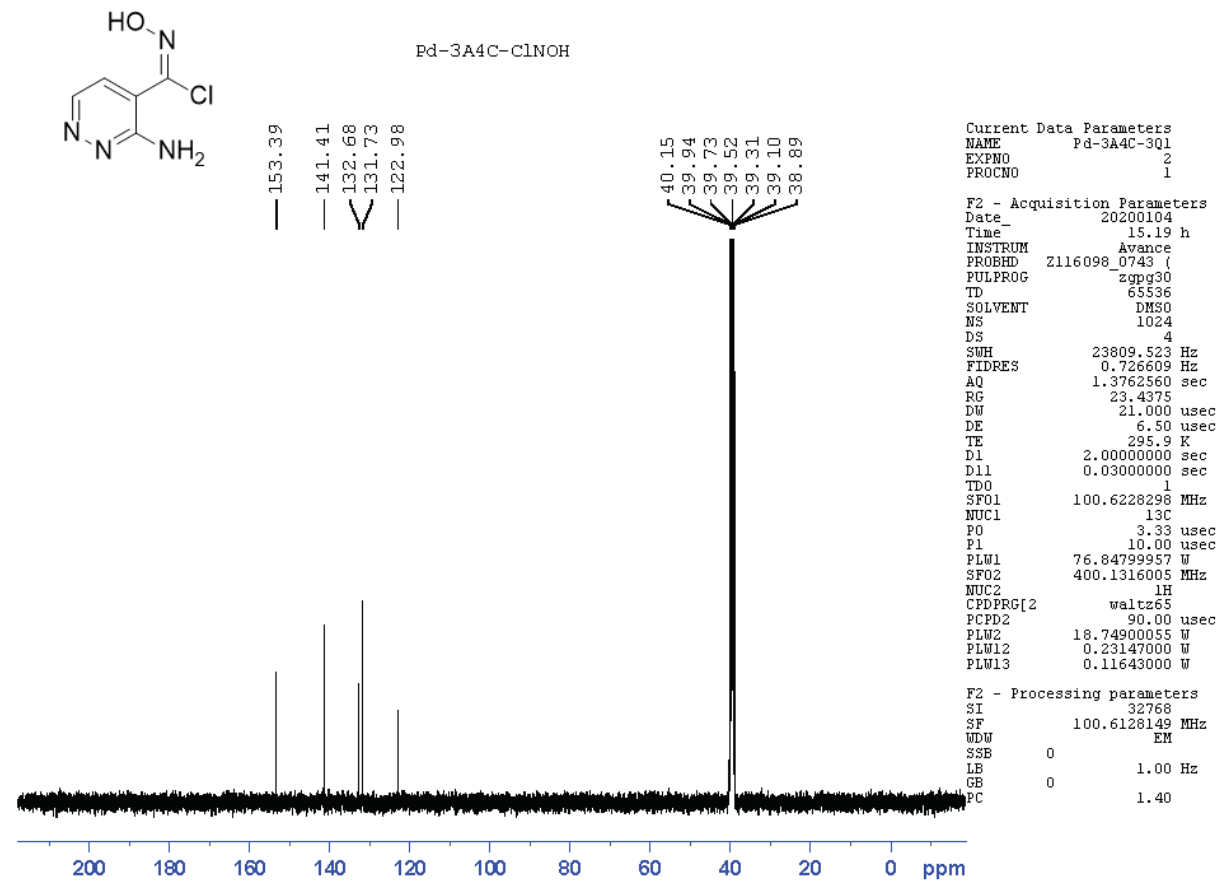

Figure S98. ${ }^{13} \mathrm{C}$ NMR of $\mathbf{5 g - 1}$ in DMSO- $\mathrm{d}_{6}$ operating at $100.61 \mathrm{MHz}$. 


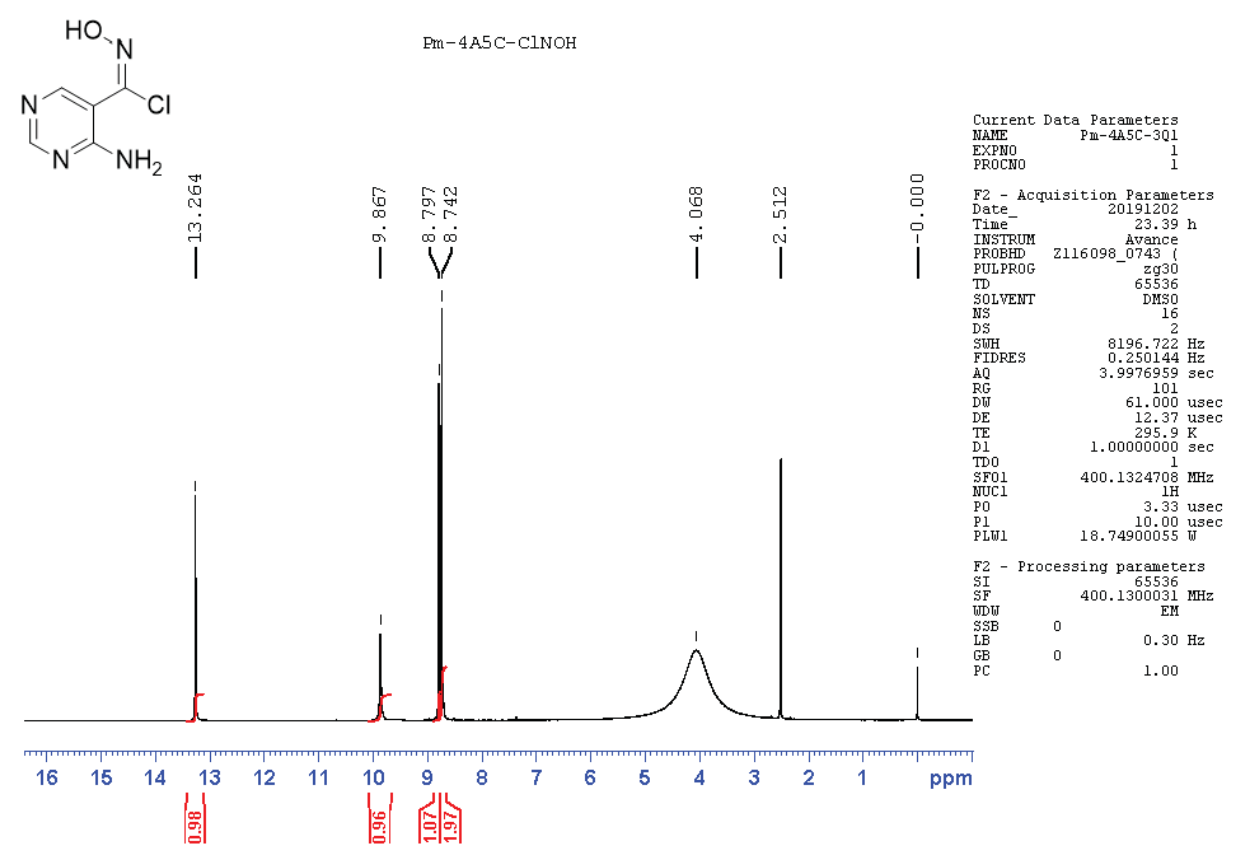

Figure S99. ${ }^{1} \mathrm{H}$ NMR of $\mathbf{5 h - 1}$ in DMSO-d 6 operating at $400.13 \mathrm{MHz}$.

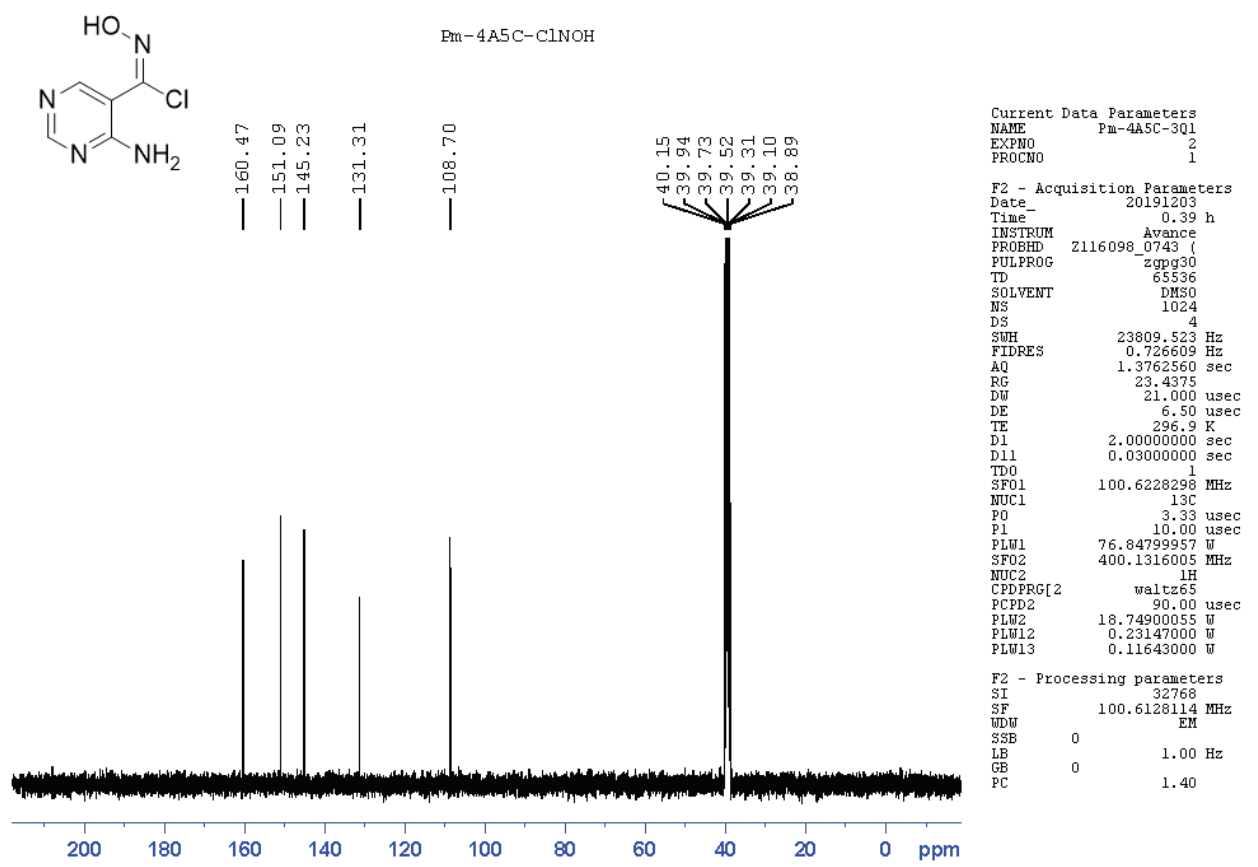

Figure S100. ${ }^{13} \mathrm{C}$ NMR of $\mathbf{5 h - 1}$ in DMSO-d 6 operating at $100.61 \mathrm{MHz}$. 


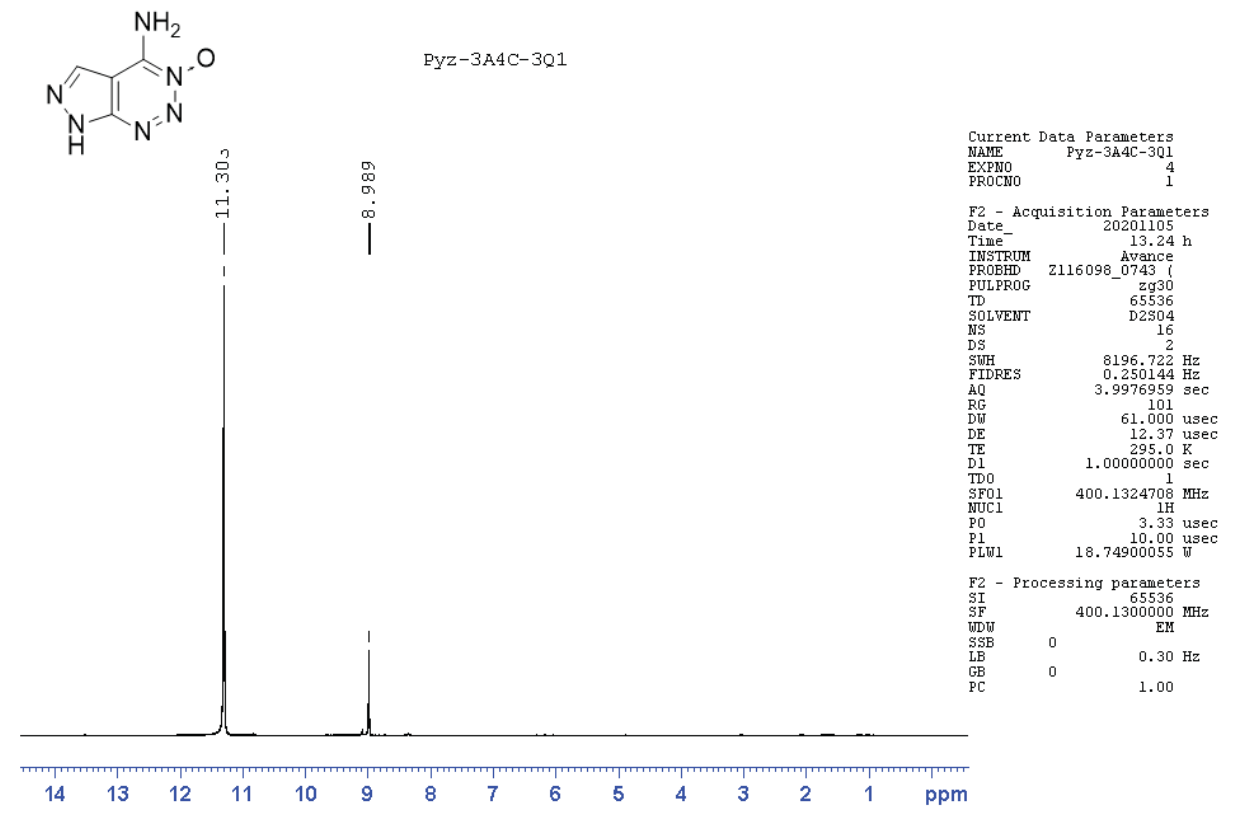

Figure $\mathrm{S} 101 .{ }^{1} \mathrm{H}$ NMR of $5 \mathbf{i}$ in $\mathrm{D}_{2} \mathrm{SO}_{4}$ operating at $400.13 \mathrm{MHz}$.

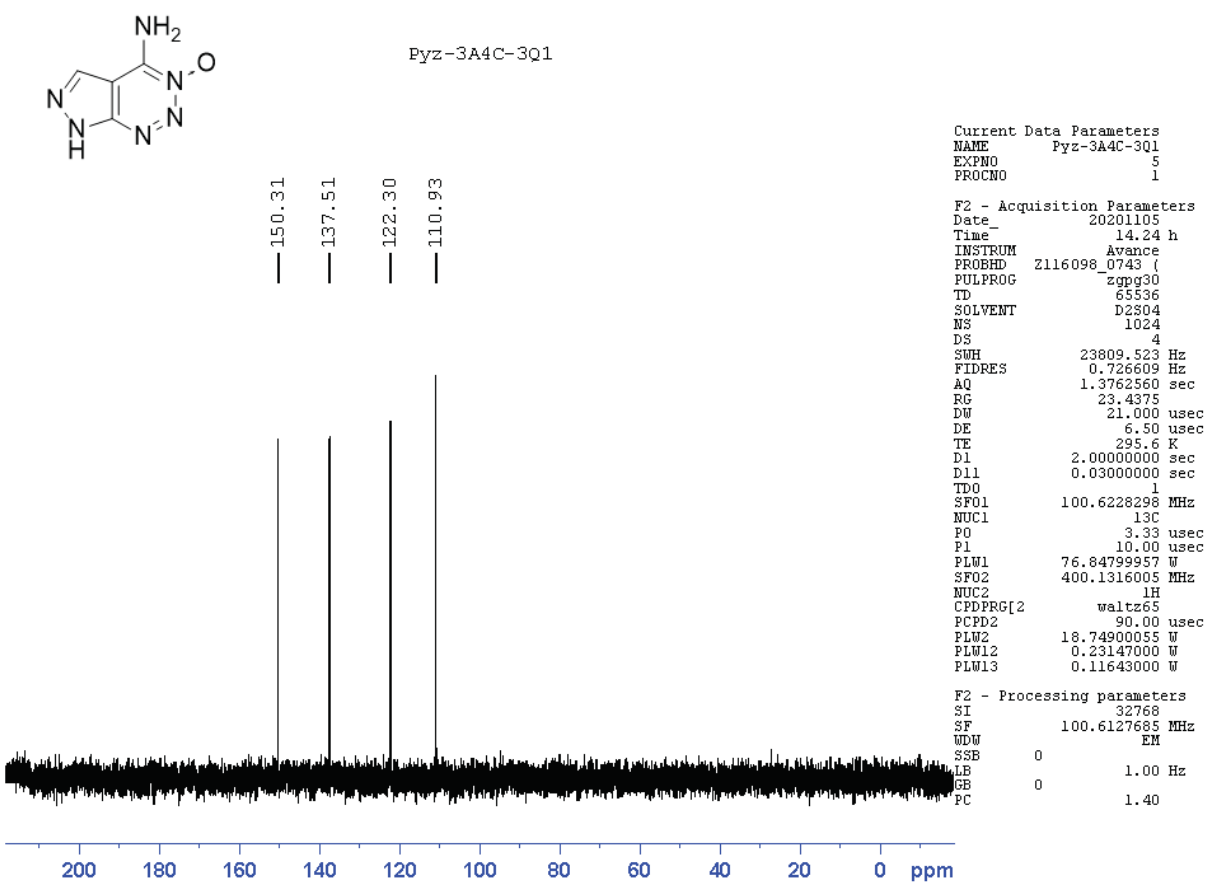

Figure $\mathrm{S} 102 .{ }^{13} \mathrm{C}$ NMR of $5 \mathbf{i}$ in $\mathrm{D}_{2} \mathrm{SO}_{4}$ operating at $100.61 \mathrm{MHz}$. 


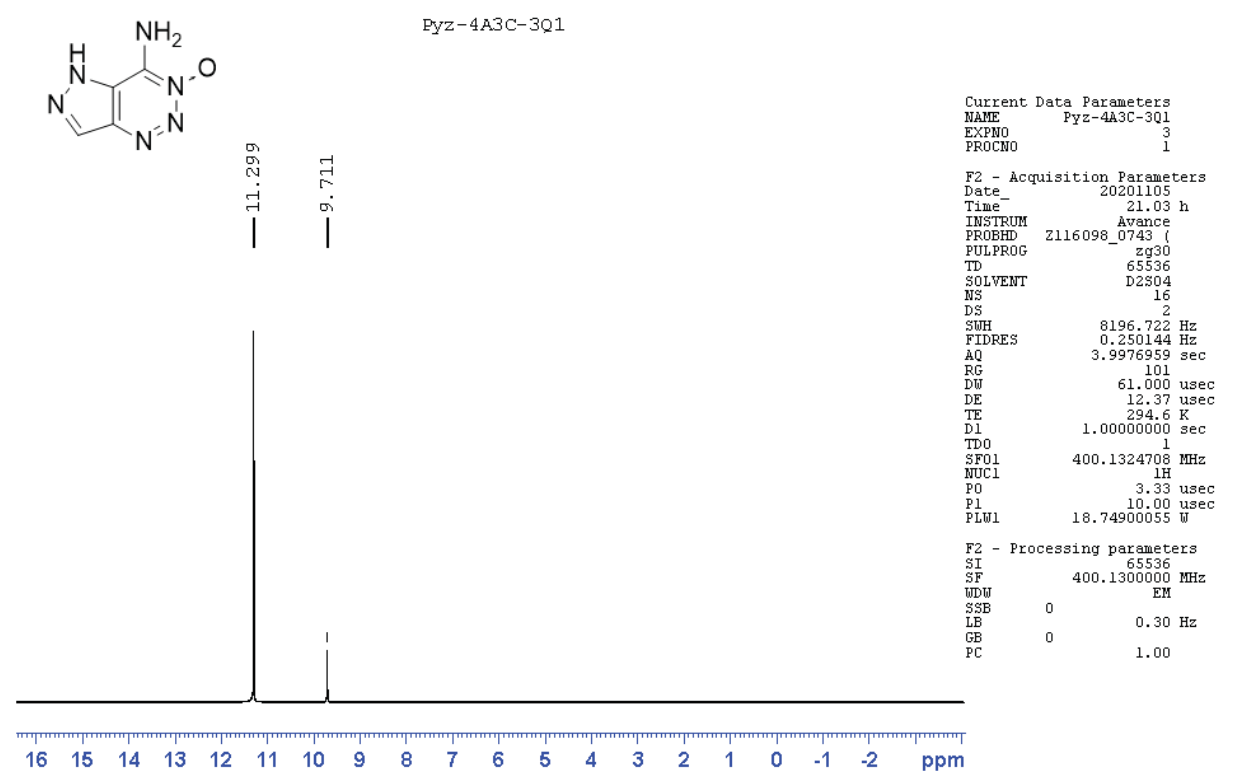

Figure $\mathrm{S} 103 .{ }^{1} \mathrm{H}$ NMR of $5 \mathrm{j}$ in $\mathrm{D}_{2} \mathrm{SO}_{4}$ operating at $400.13 \mathrm{MHz}$.

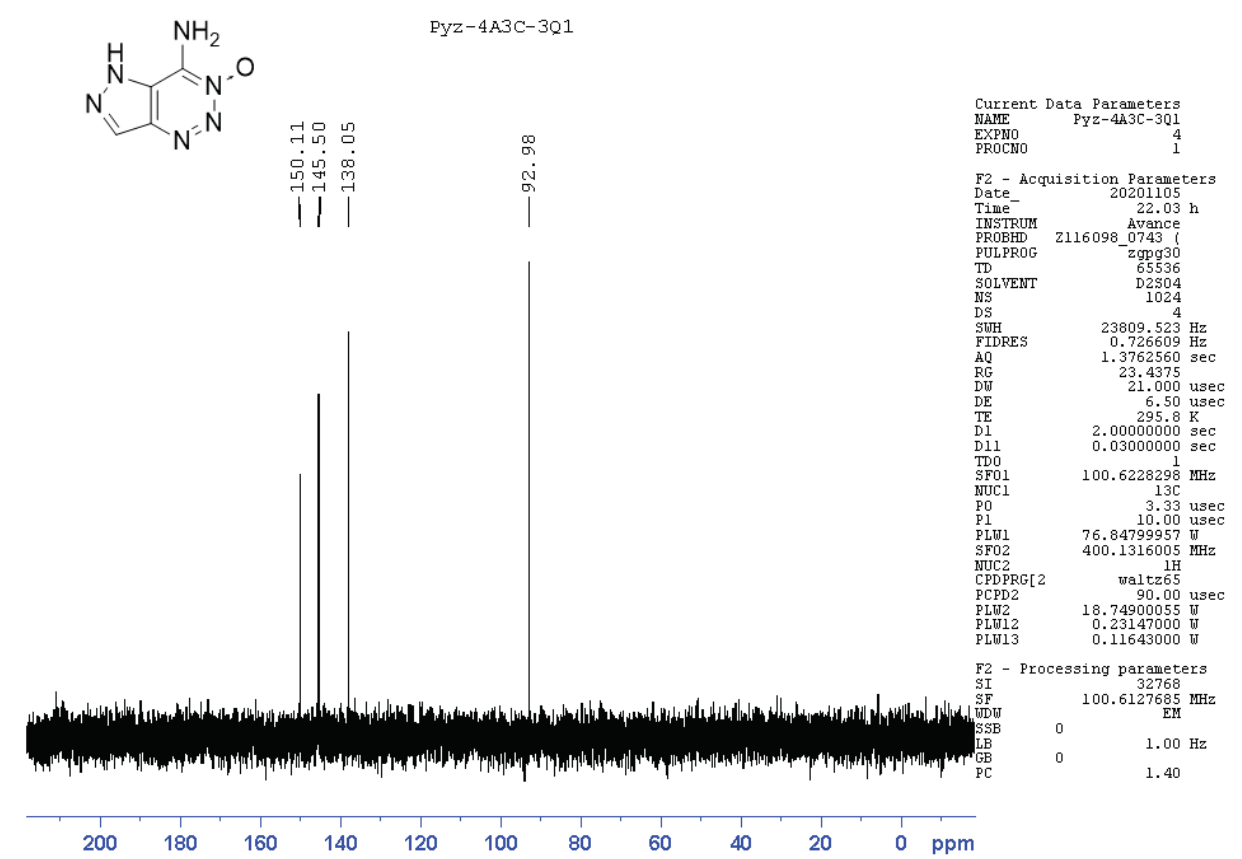

Figure $\mathrm{S} 104 .{ }^{13} \mathrm{C}$ NMR of $5 \mathbf{j}$ in $\mathrm{D}_{2} \mathrm{SO}_{4}$ operating at $100.61 \mathrm{MHz}$. 


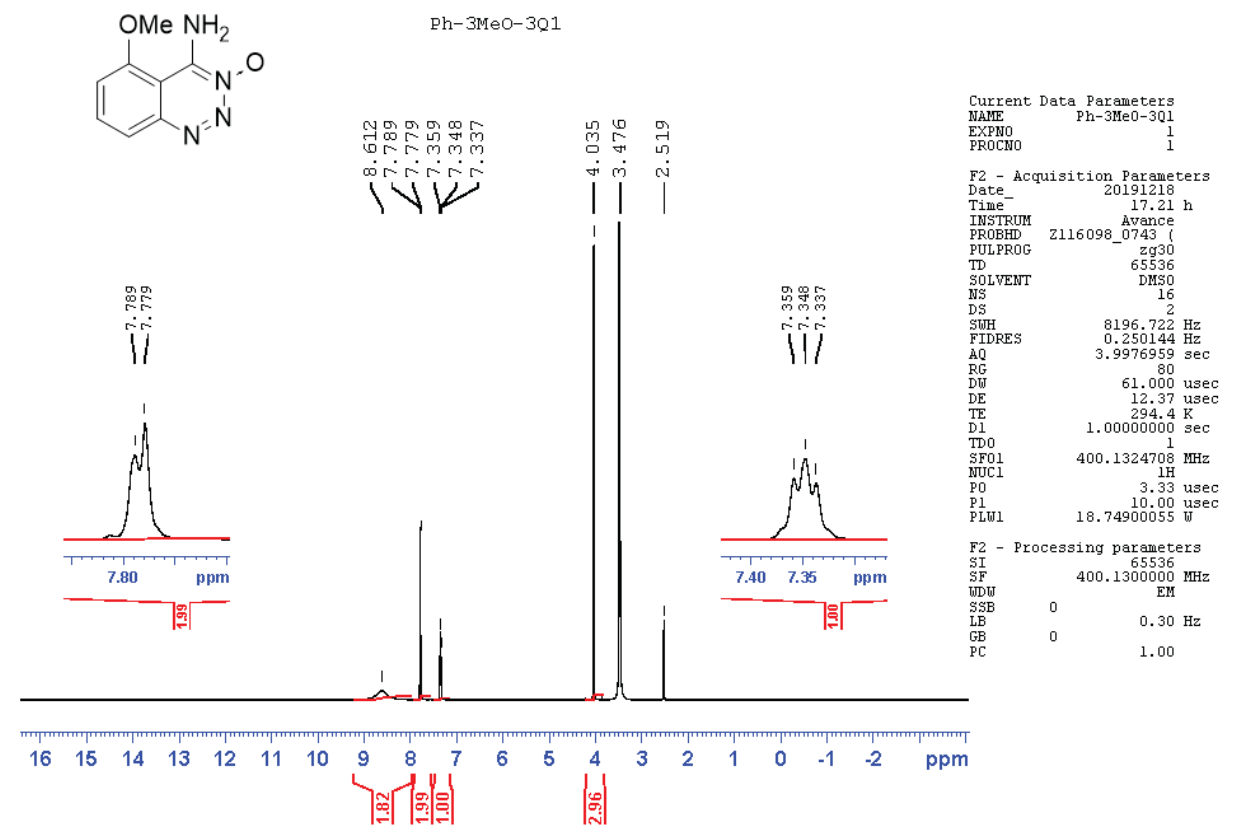

Figure $\mathrm{S} 105 .{ }^{1} \mathrm{H}$ NMR of $\mathbf{5 k}$ in DMSO- $\mathrm{d}_{6}$ operating at $400.13 \mathrm{MHz}$.

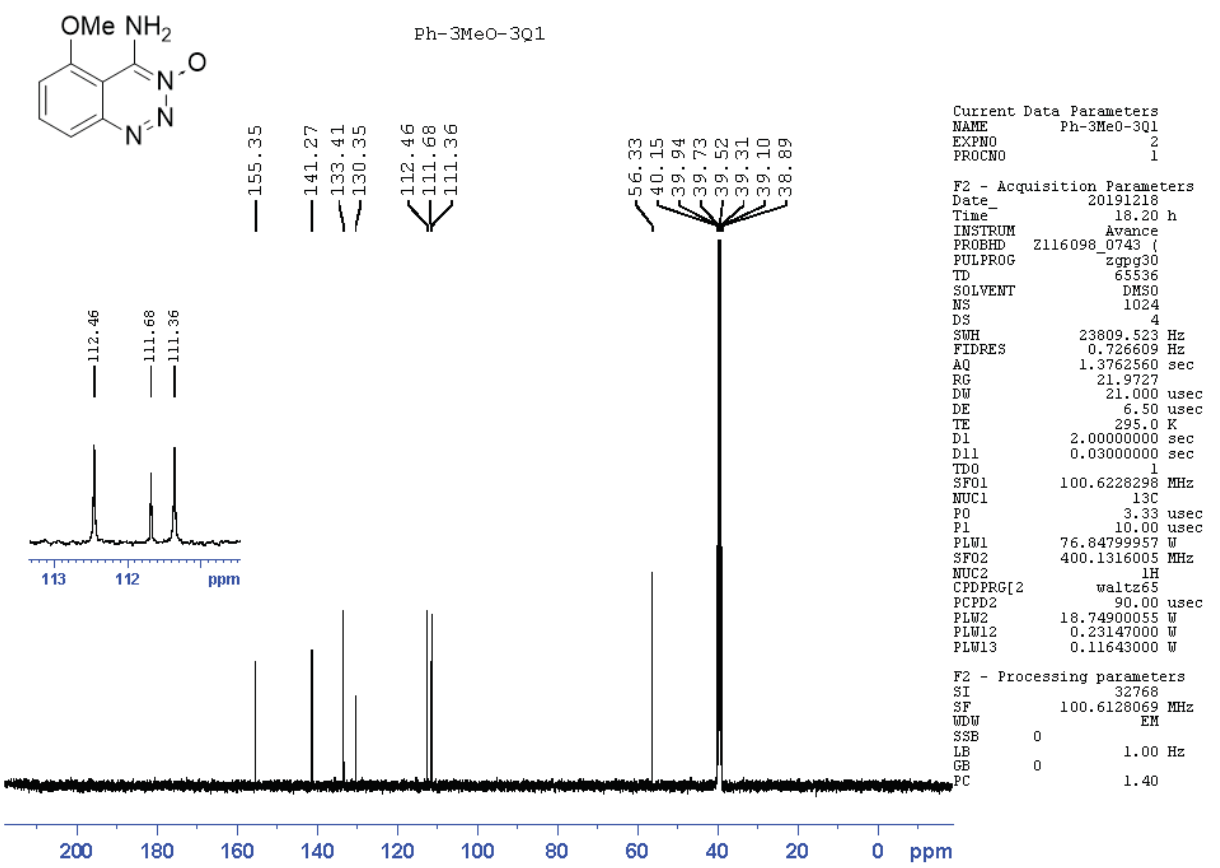

Figure S106. ${ }^{13} \mathrm{C}$ NMR of $5 \mathbf{k}$ in DMSO-d 6 operating at $100.61 \mathrm{MHz}$. 


\section{HRMS Spectra}
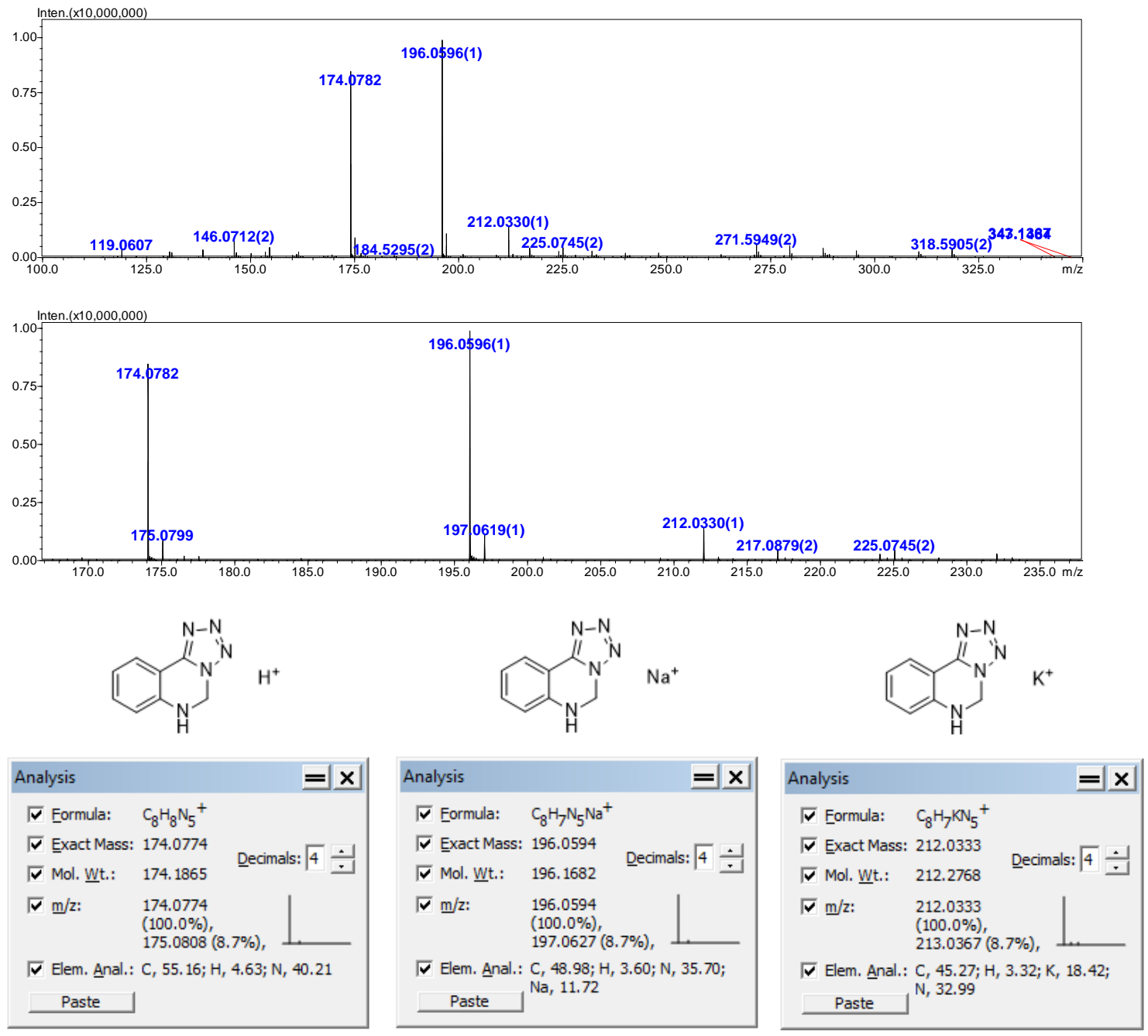

Figure S107. HRMS(ESI+) of 2a-1.

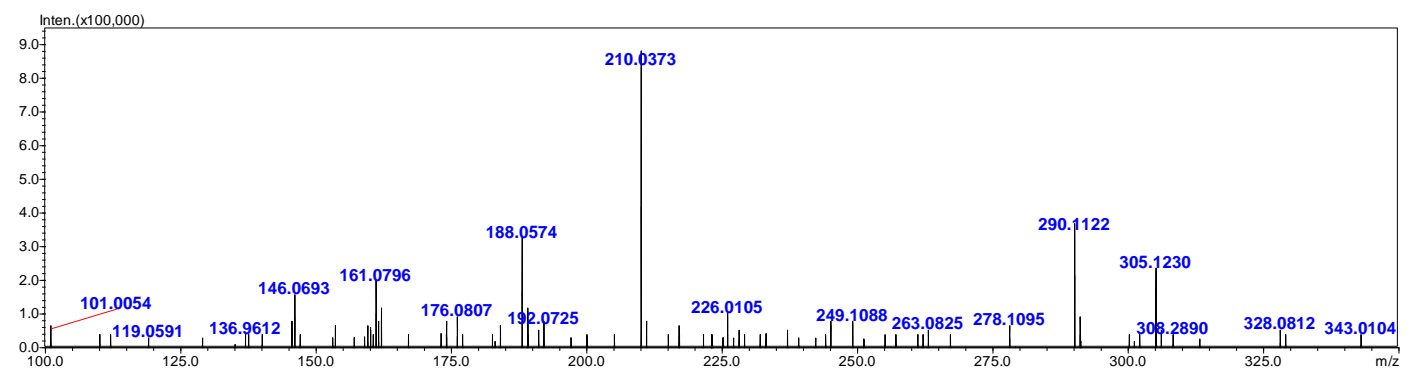



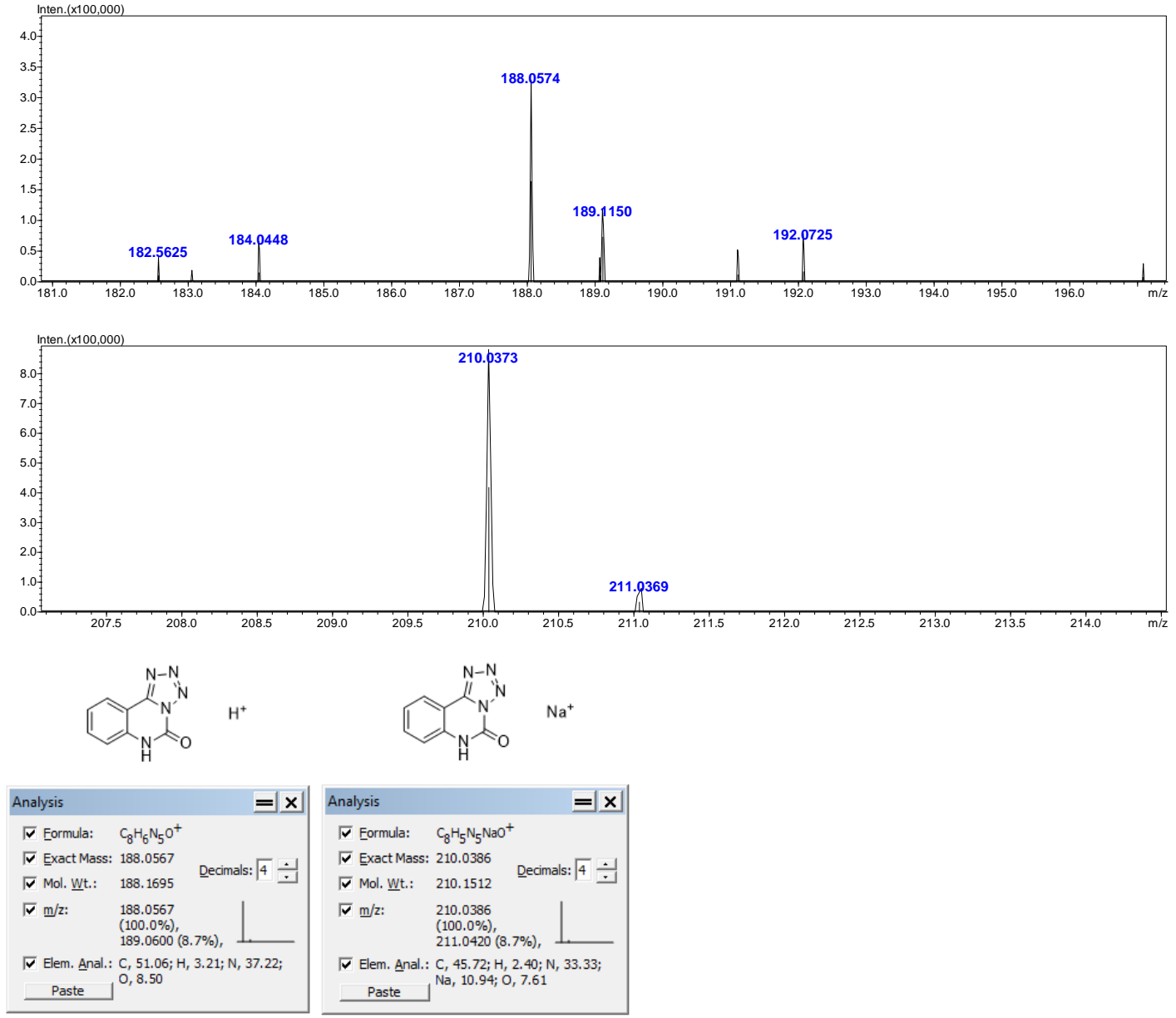

Figure S108. HRMS(ESI+) of 2a-2.
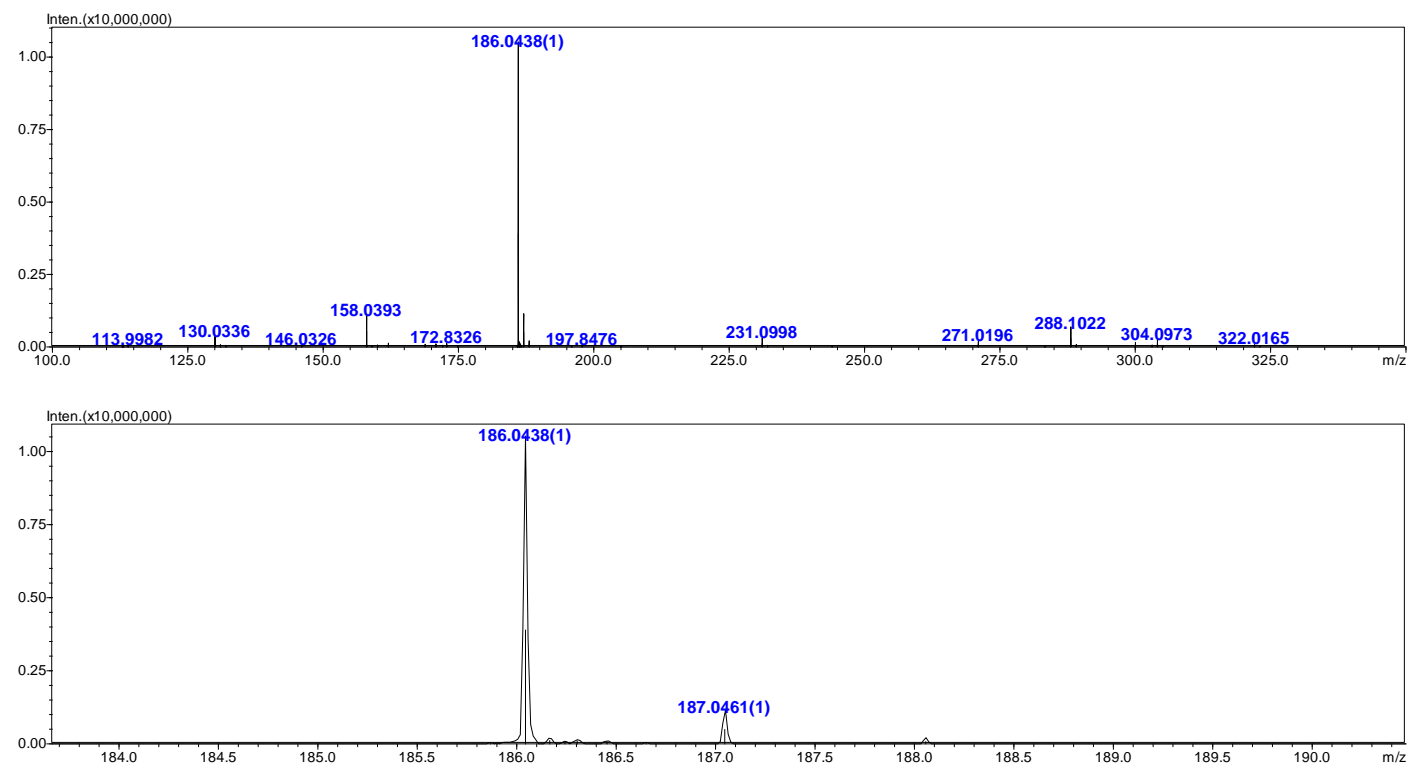


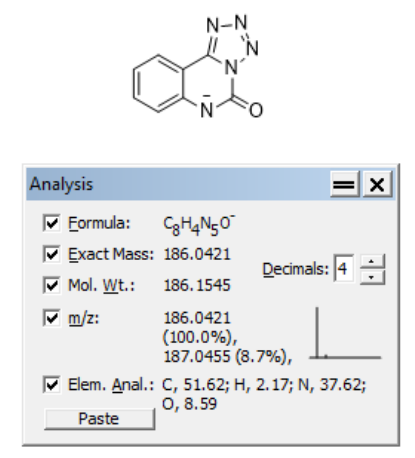

Figure S109. HRMS(ESI-) of 2a-2.
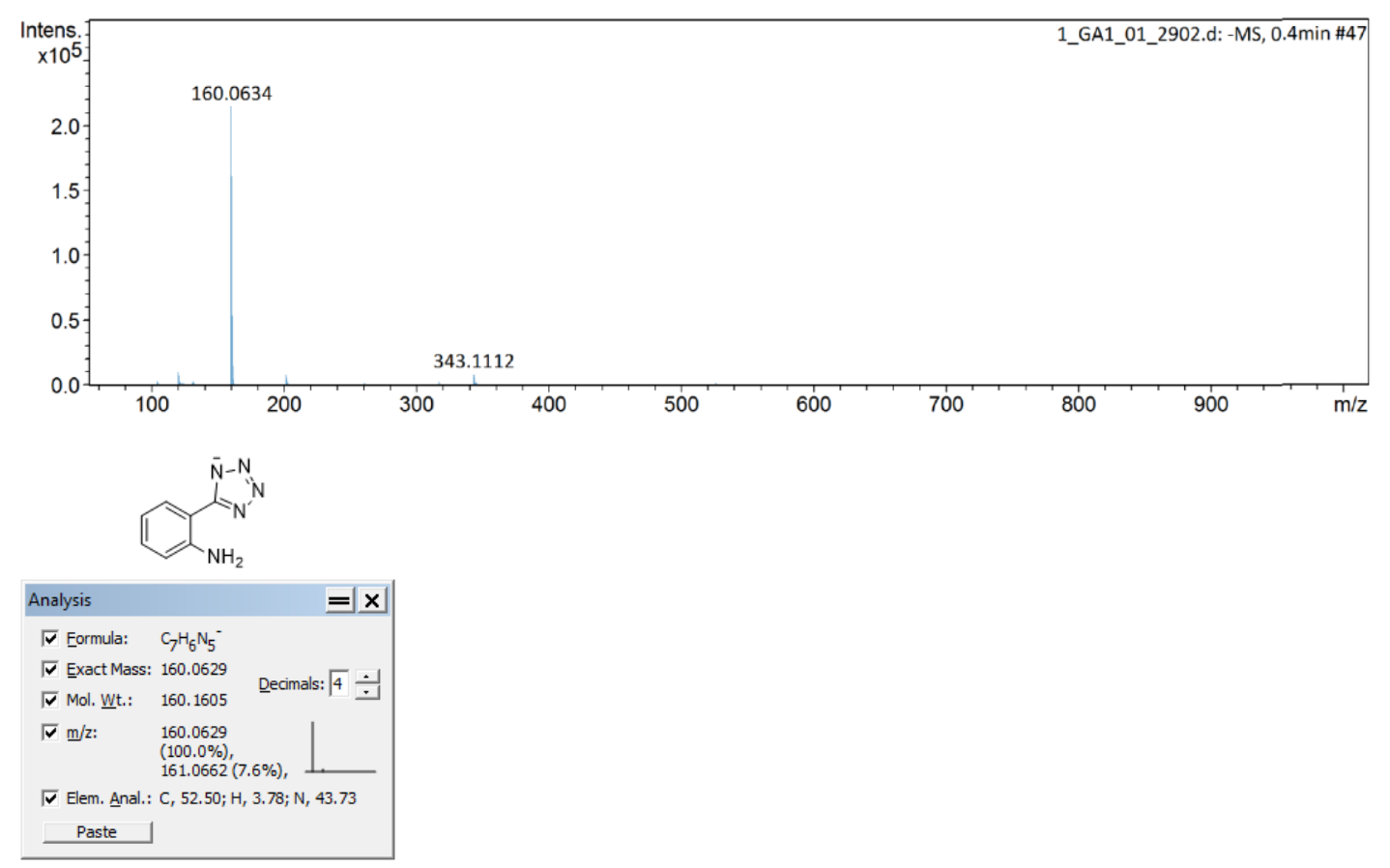

Figure S110. HRMS(ESI-) of 2a.

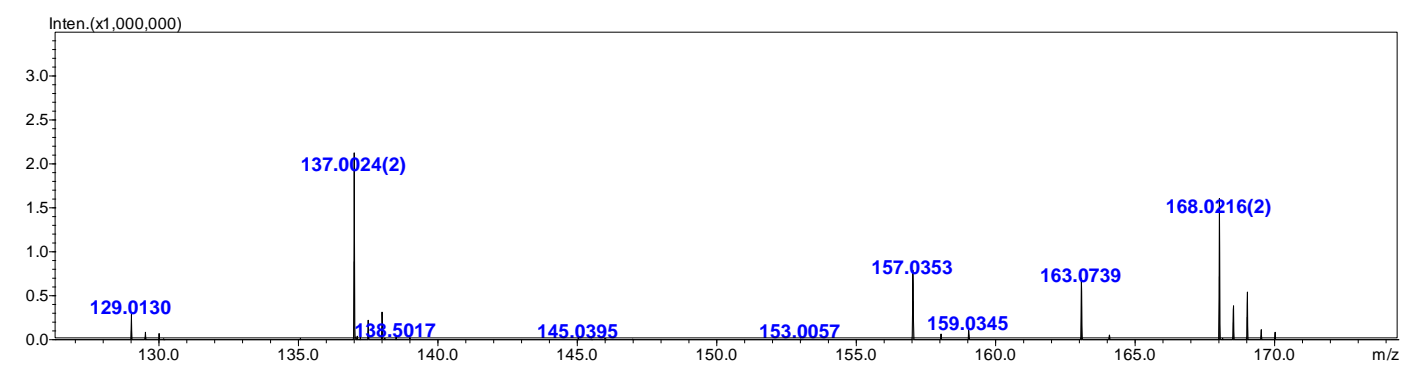



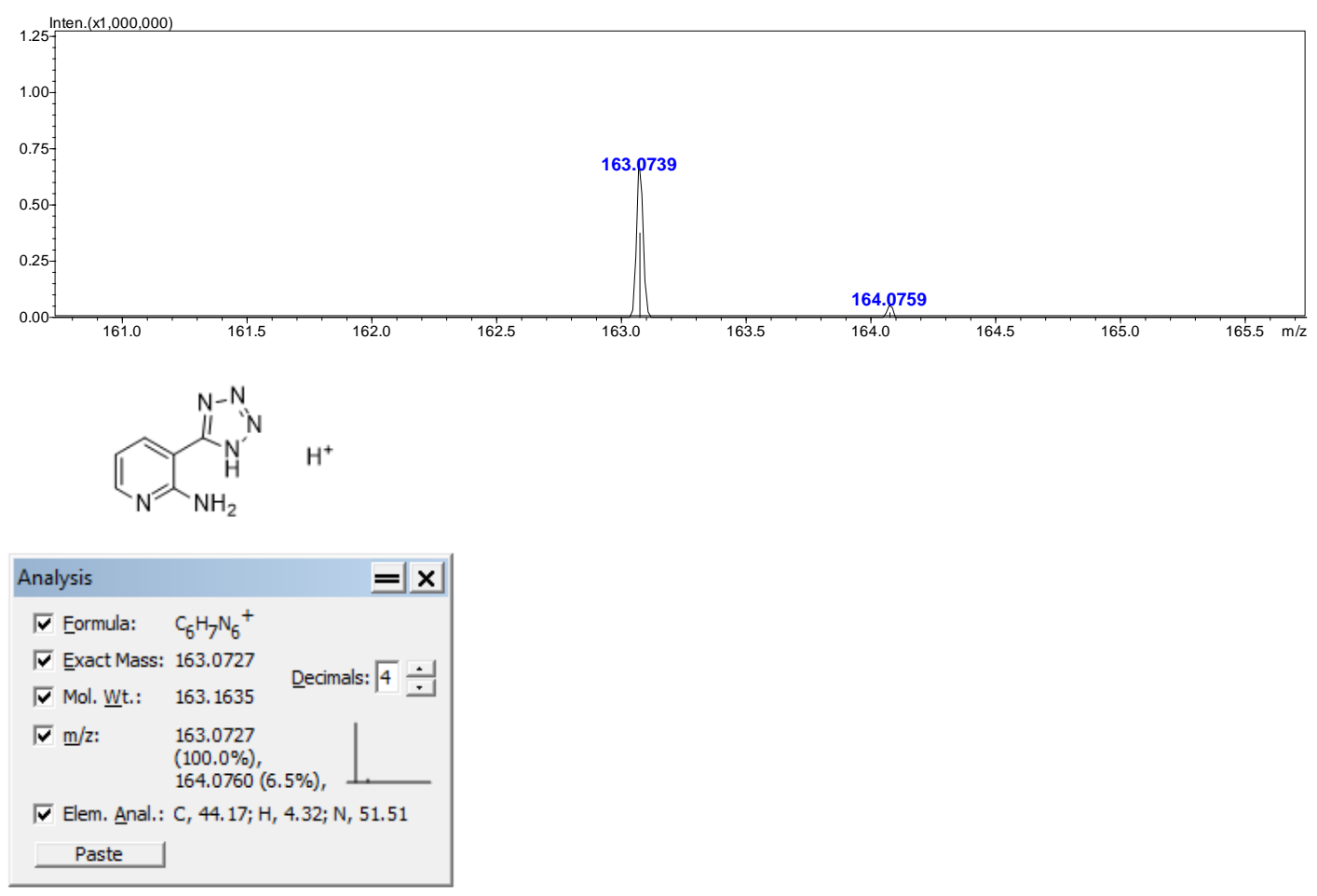

Figure S111. HRMS(ESI+) of $\mathbf{2 b}$.
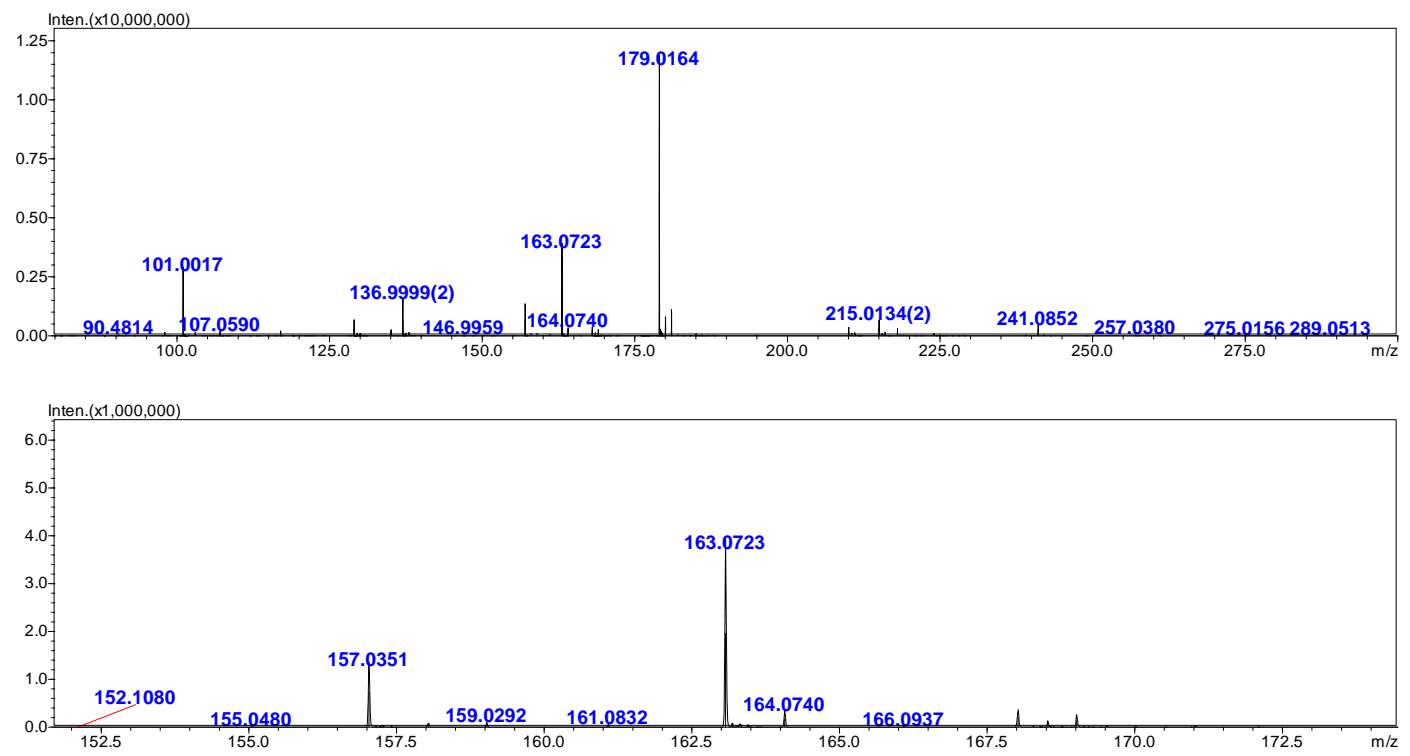


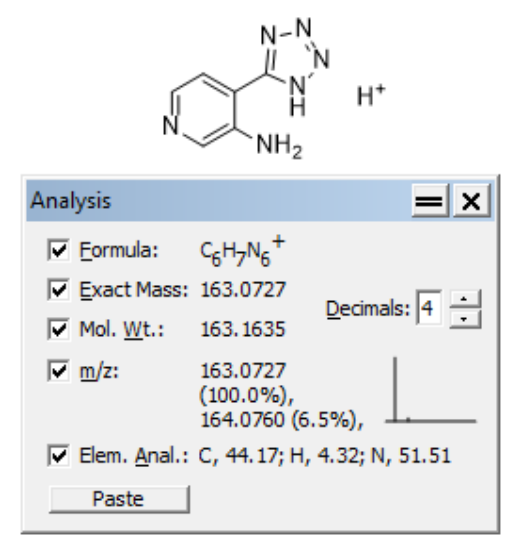

Figure S112. HRMS(ESI+) of 2c.
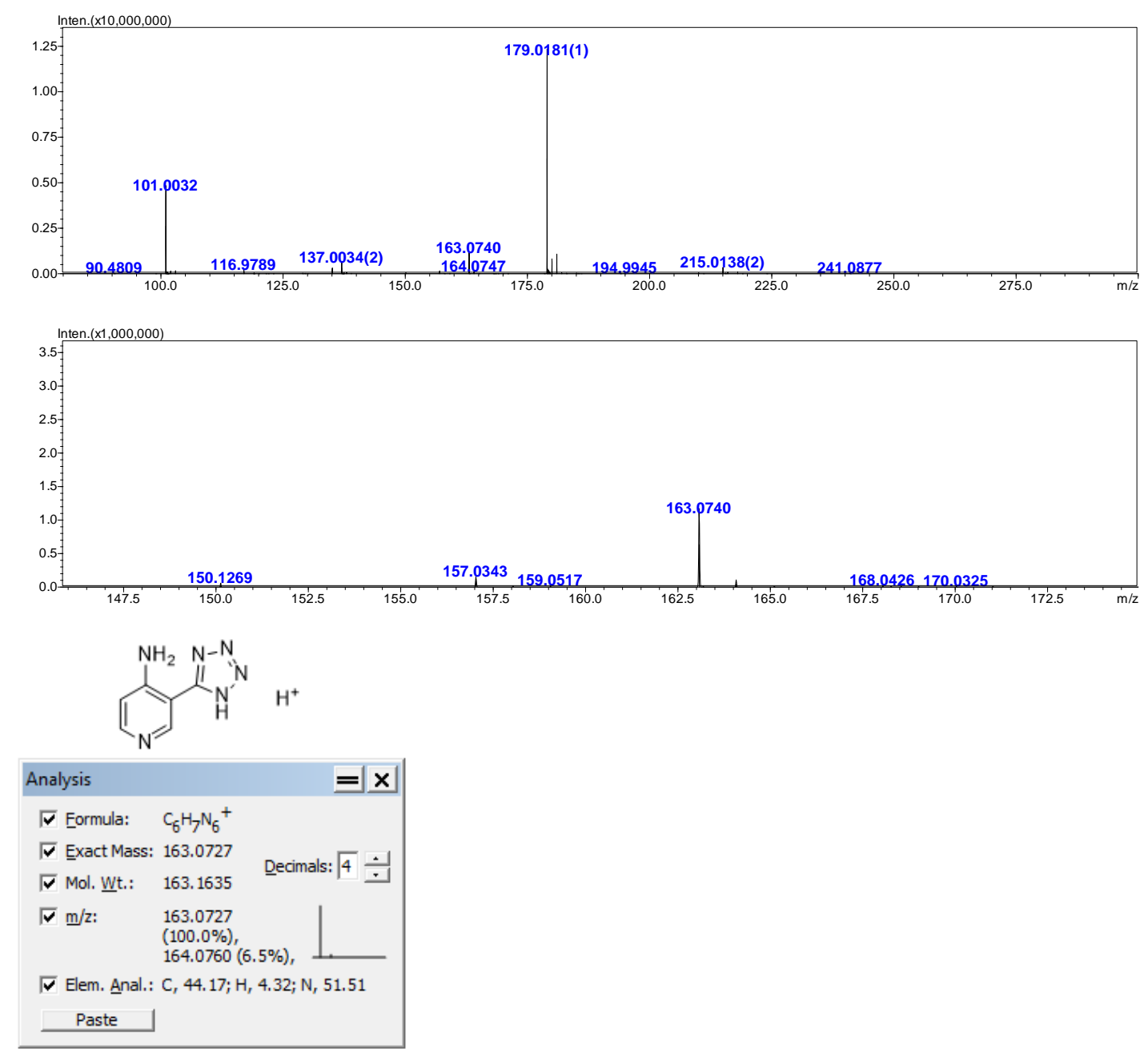

Figure S113. HRMS(ESI+) of $\mathbf{2 d}$. 

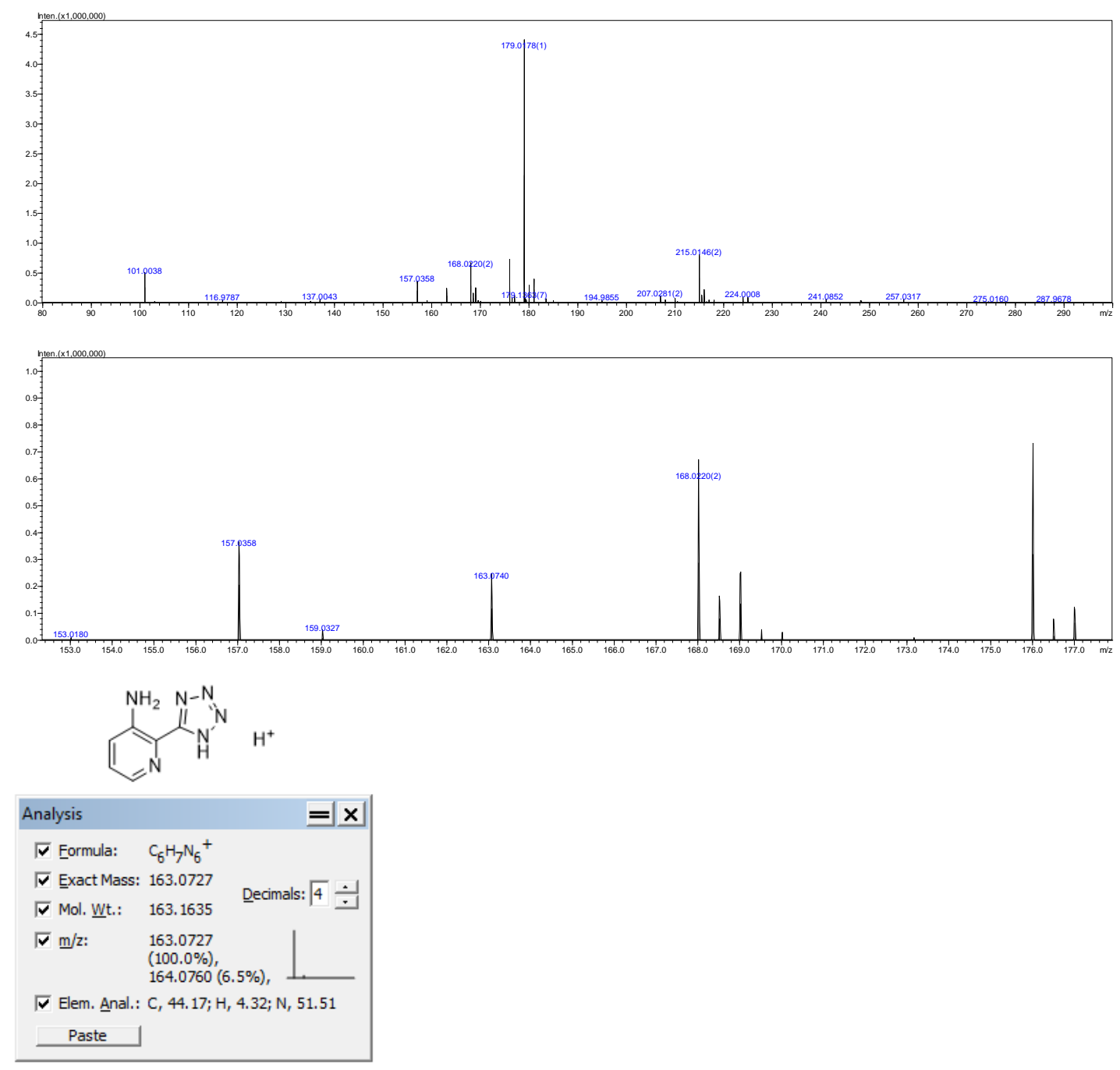

Figure S114. HRMS(ESI+) of $2 \mathbf{e}$.

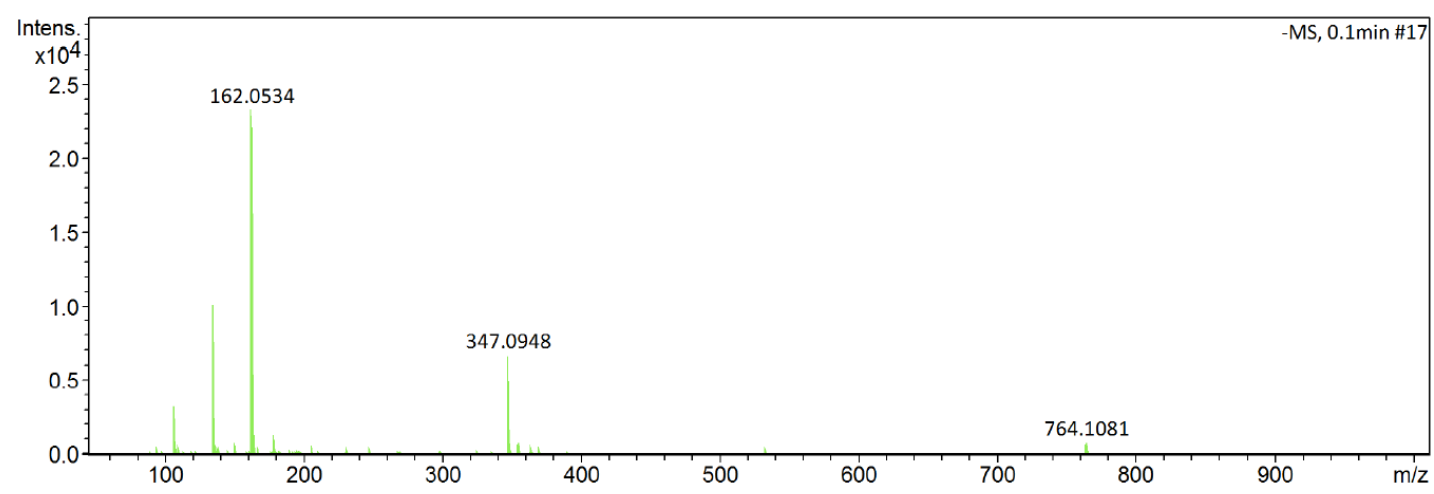




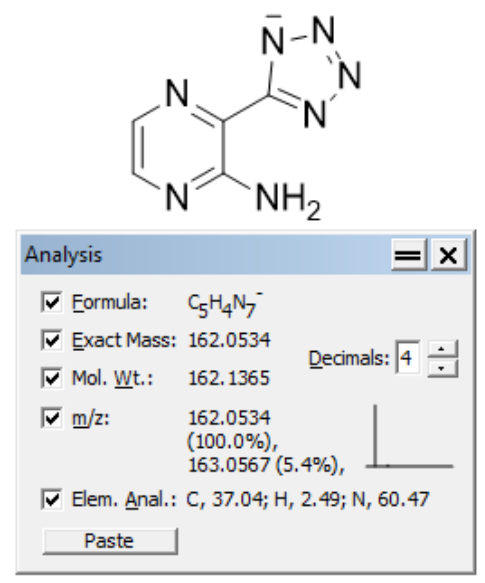

Figure S115. HRMS(ESI-) of $\mathbf{2 f}$.
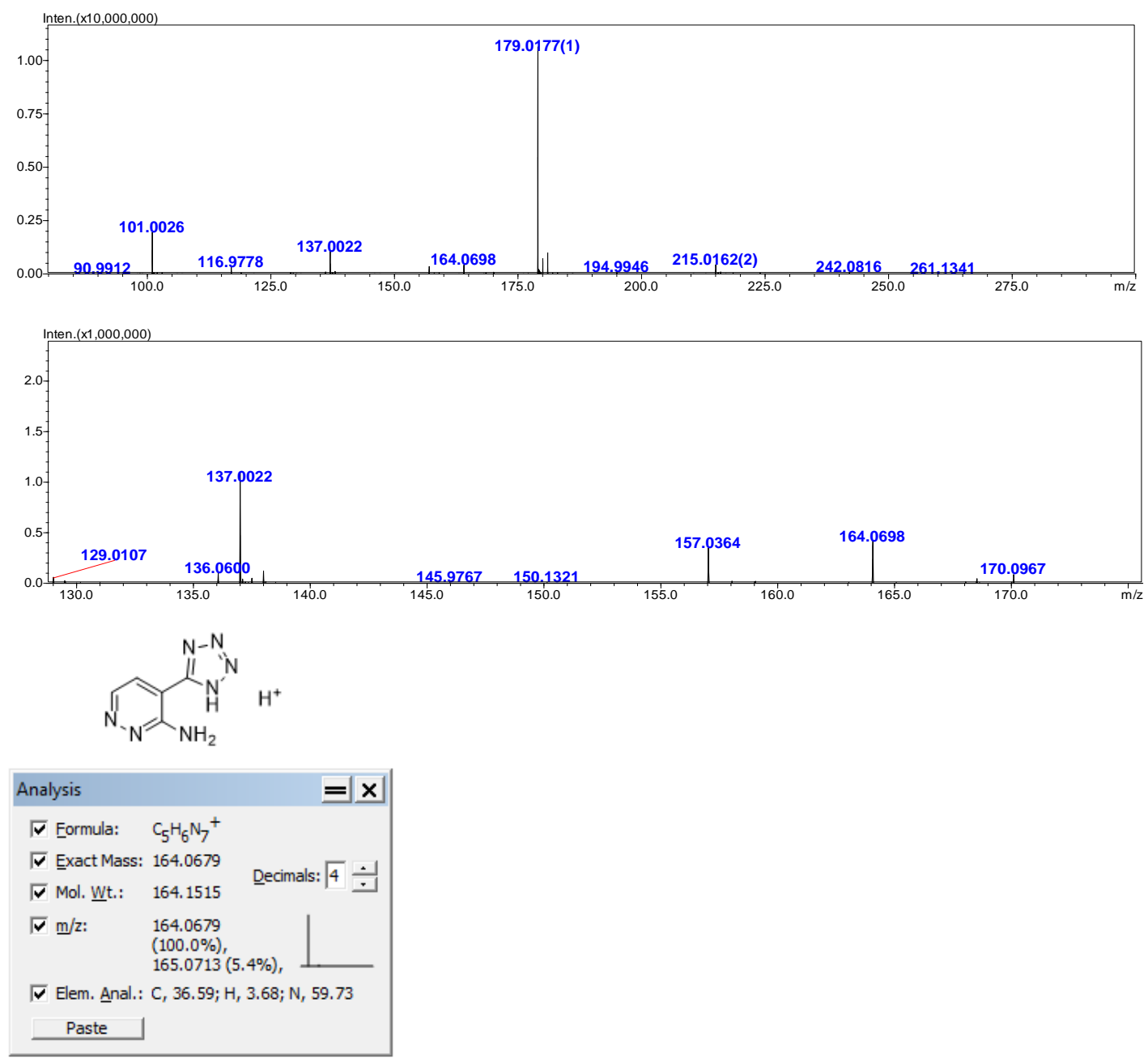

Figure S116. HRMS(ESI+) of $\mathbf{2 g}$. 


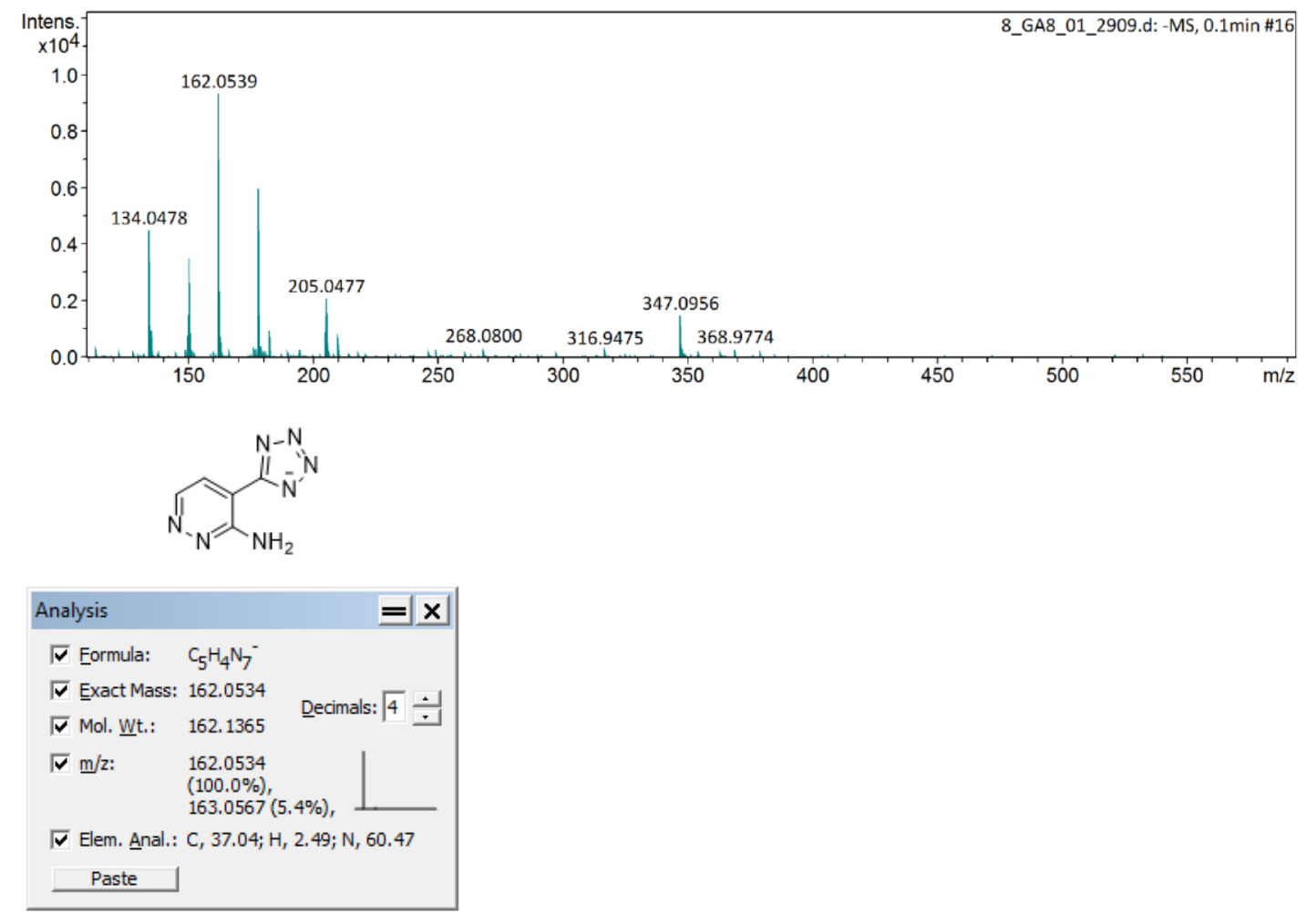

Figure S117. HRMS(ESI-) of $\mathbf{2 g}$.
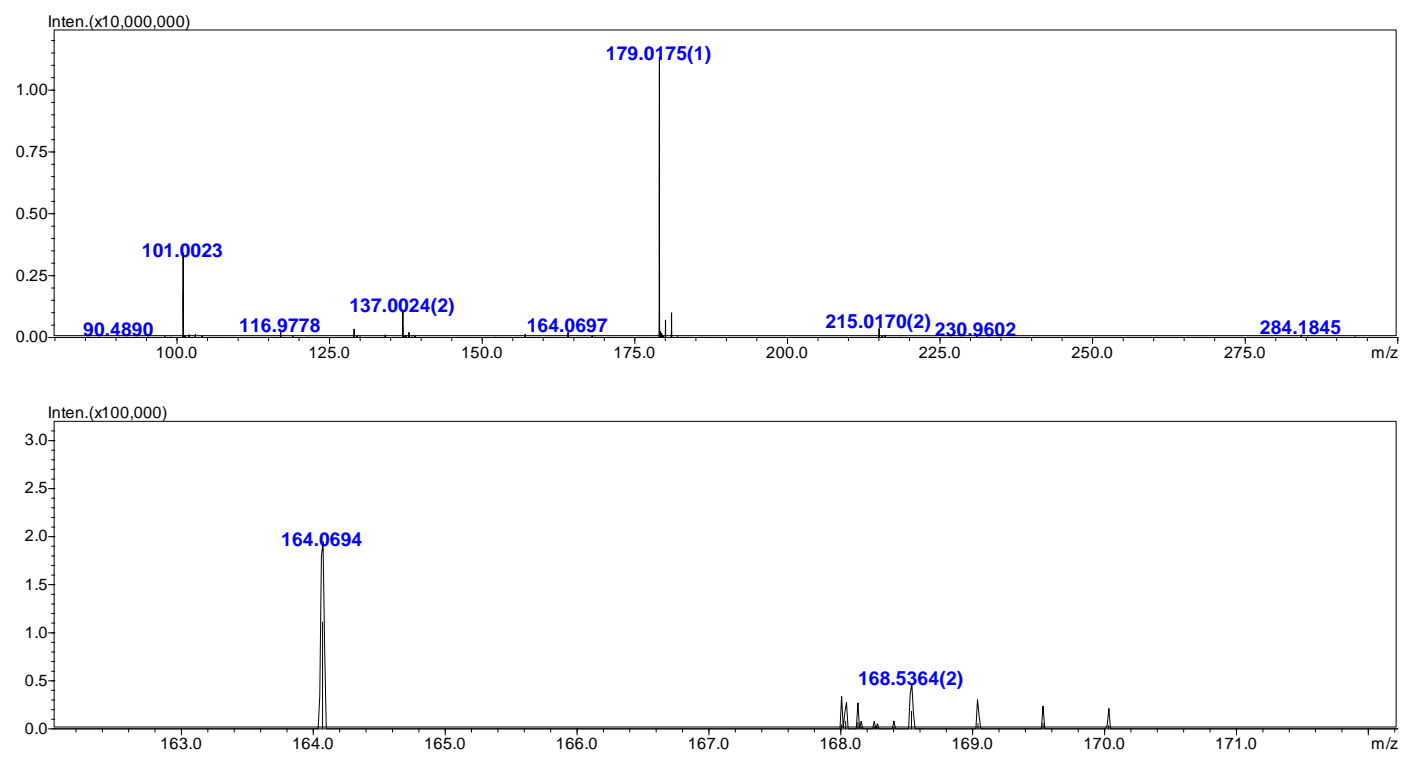

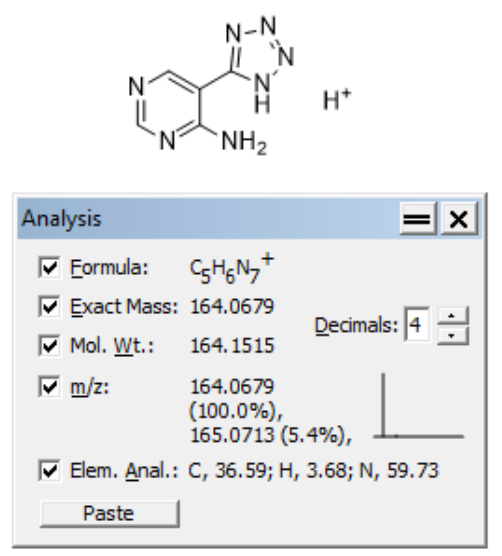

Figure S118. HRMS(ESI+) of $\mathbf{2 h}$.
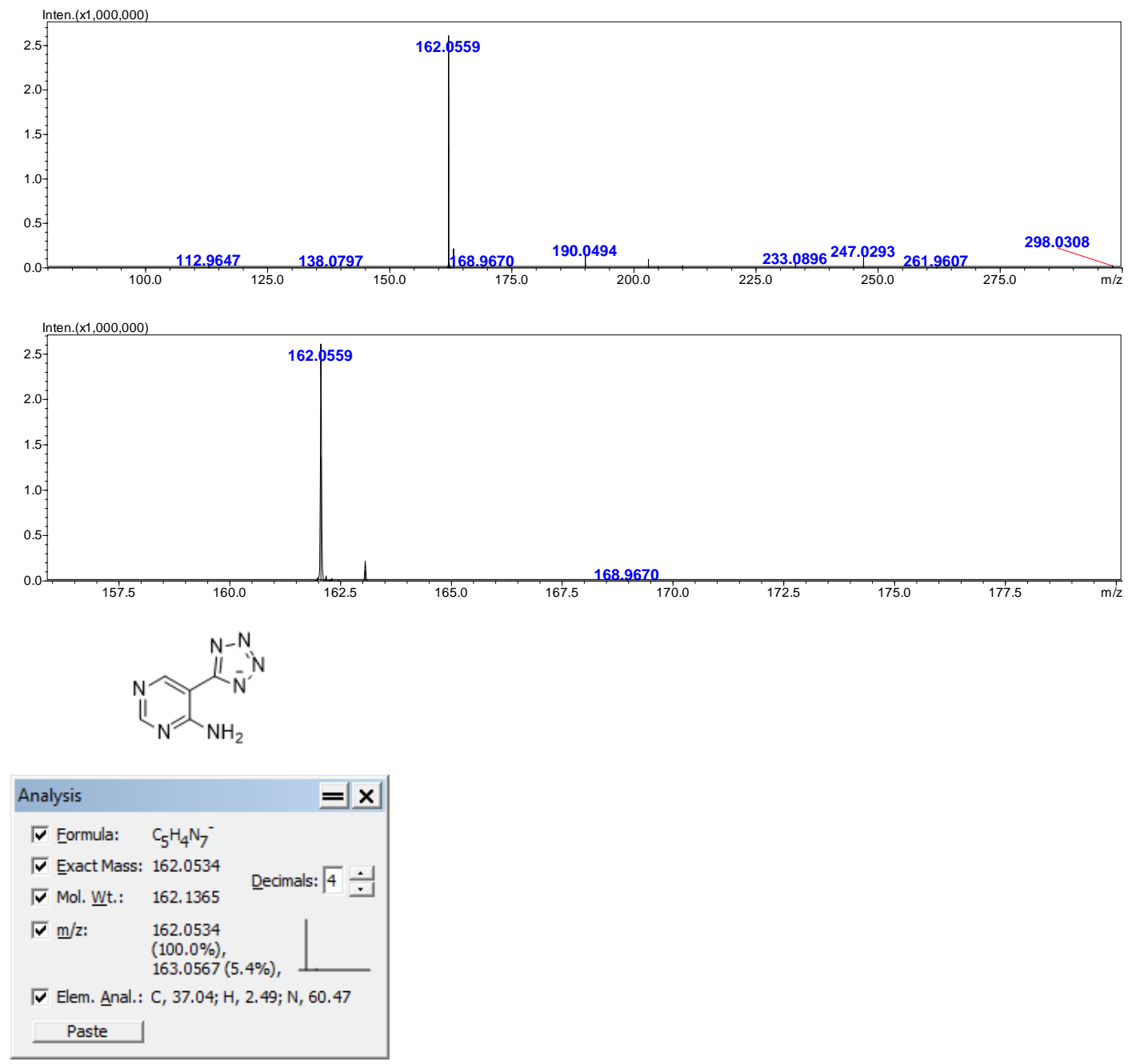

Figure S119. HRMS(ESI-) of $\mathbf{2 h}$. 


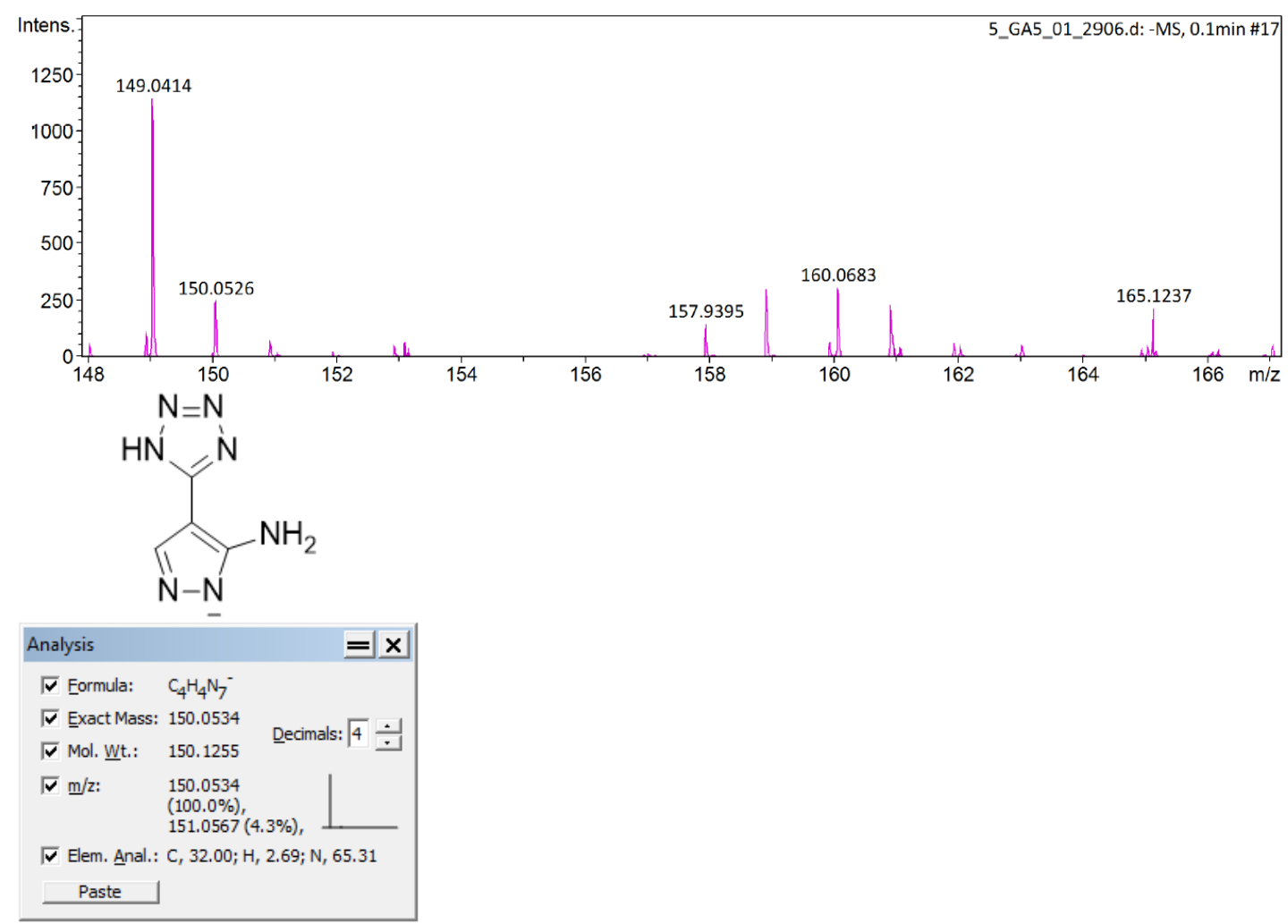

Figure S120. HRMS(ESI-) of $\mathbf{2} \mathbf{i}$.
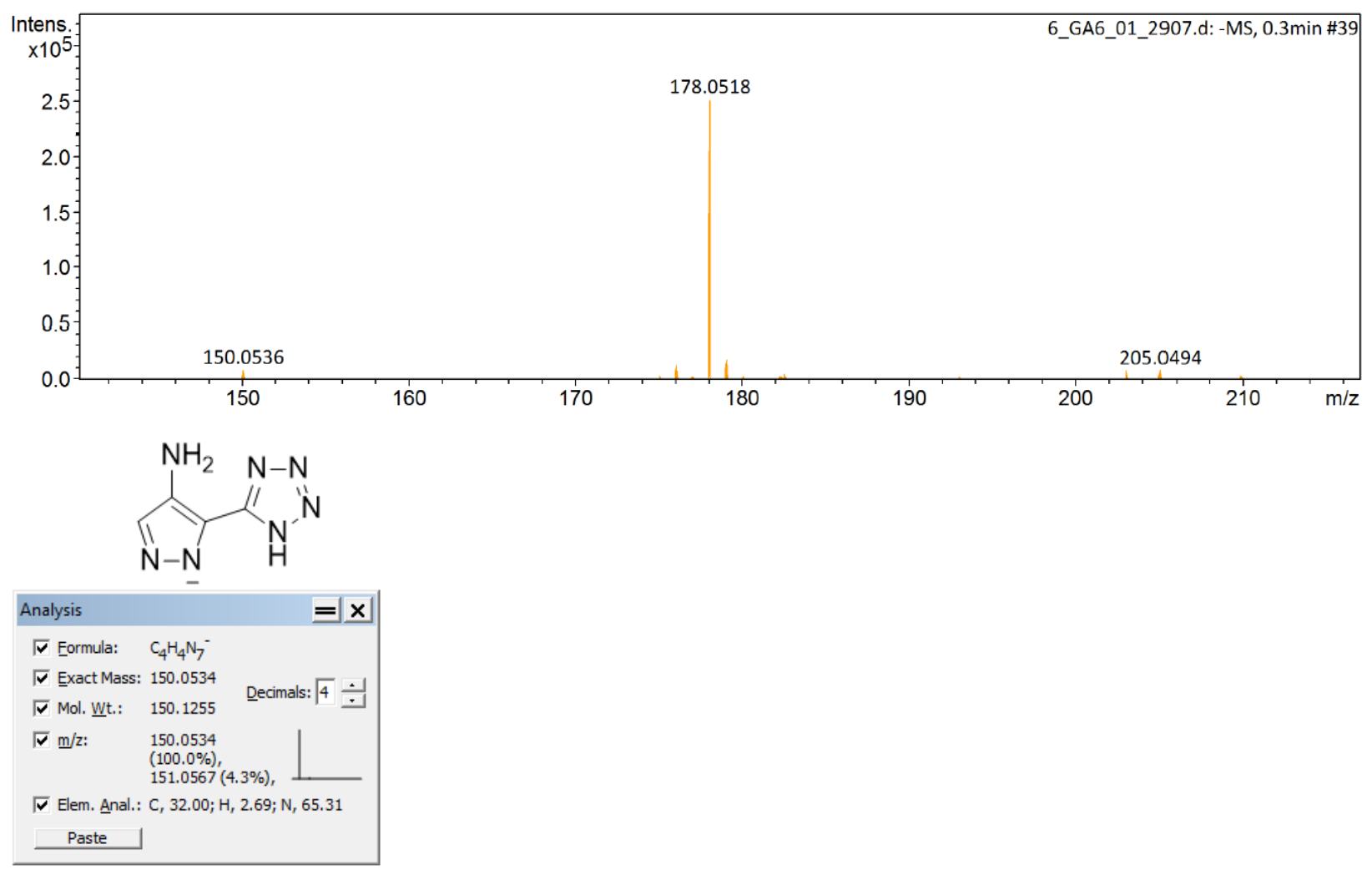

Figure S121. HRMS(ESI-) of $\mathbf{2 j}$. 

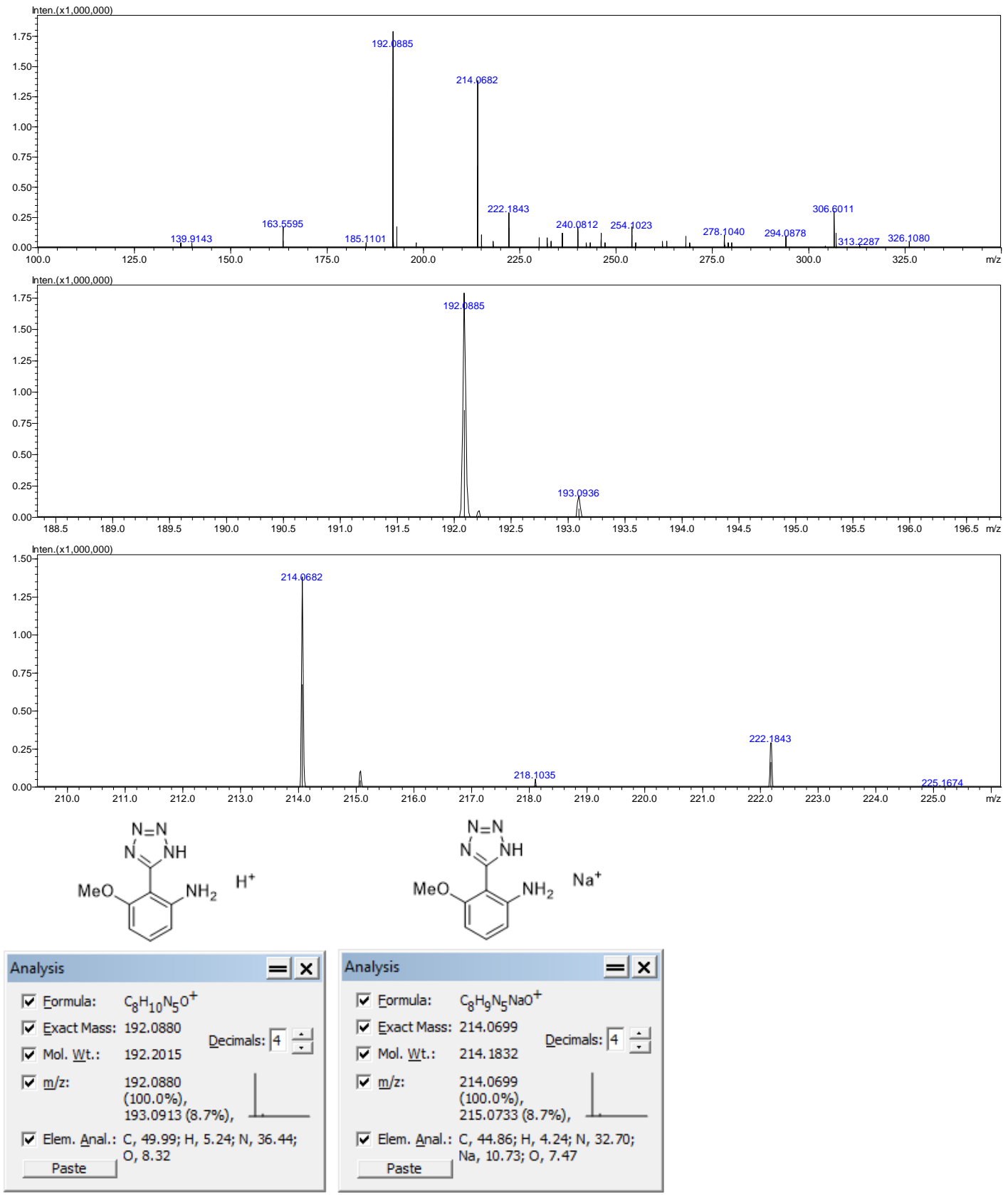

Figure S122. HRMS (ESI+) of $\mathbf{2 k}$.

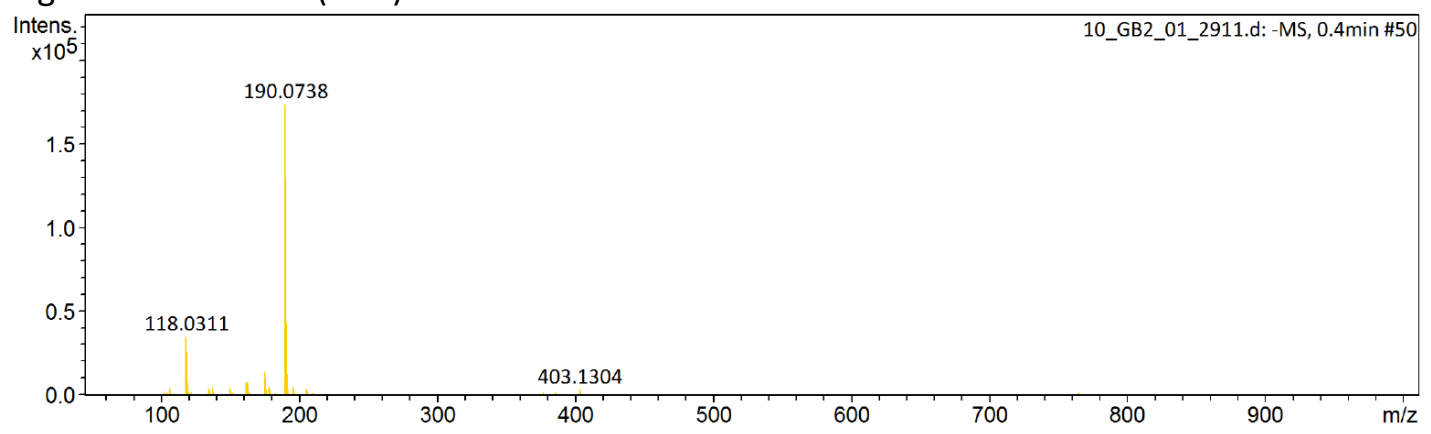




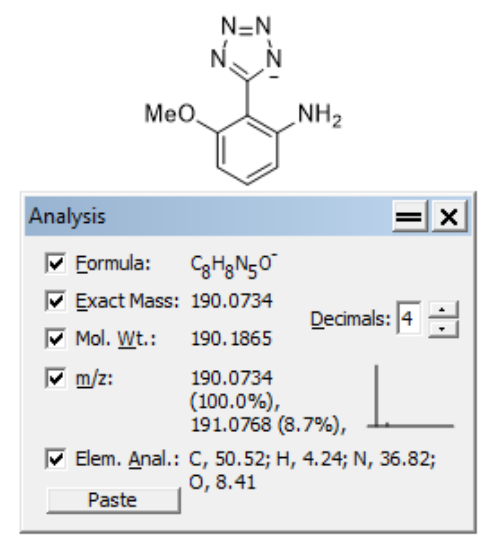

Figure S123. HRMS (ESI-) of $\mathbf{2 k}$.
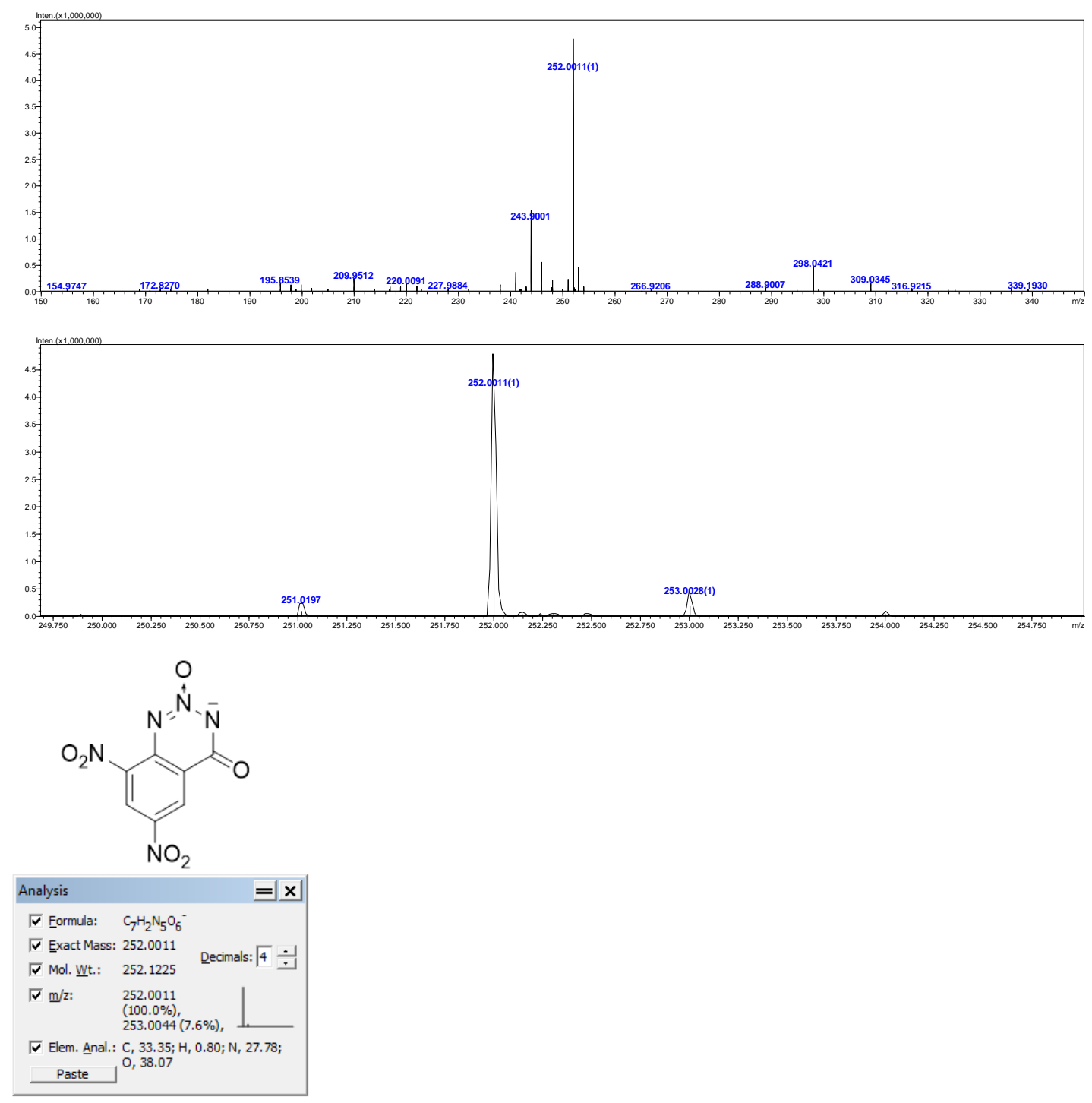

Figure S124. HRMS(ESI-) of 3a-1. 

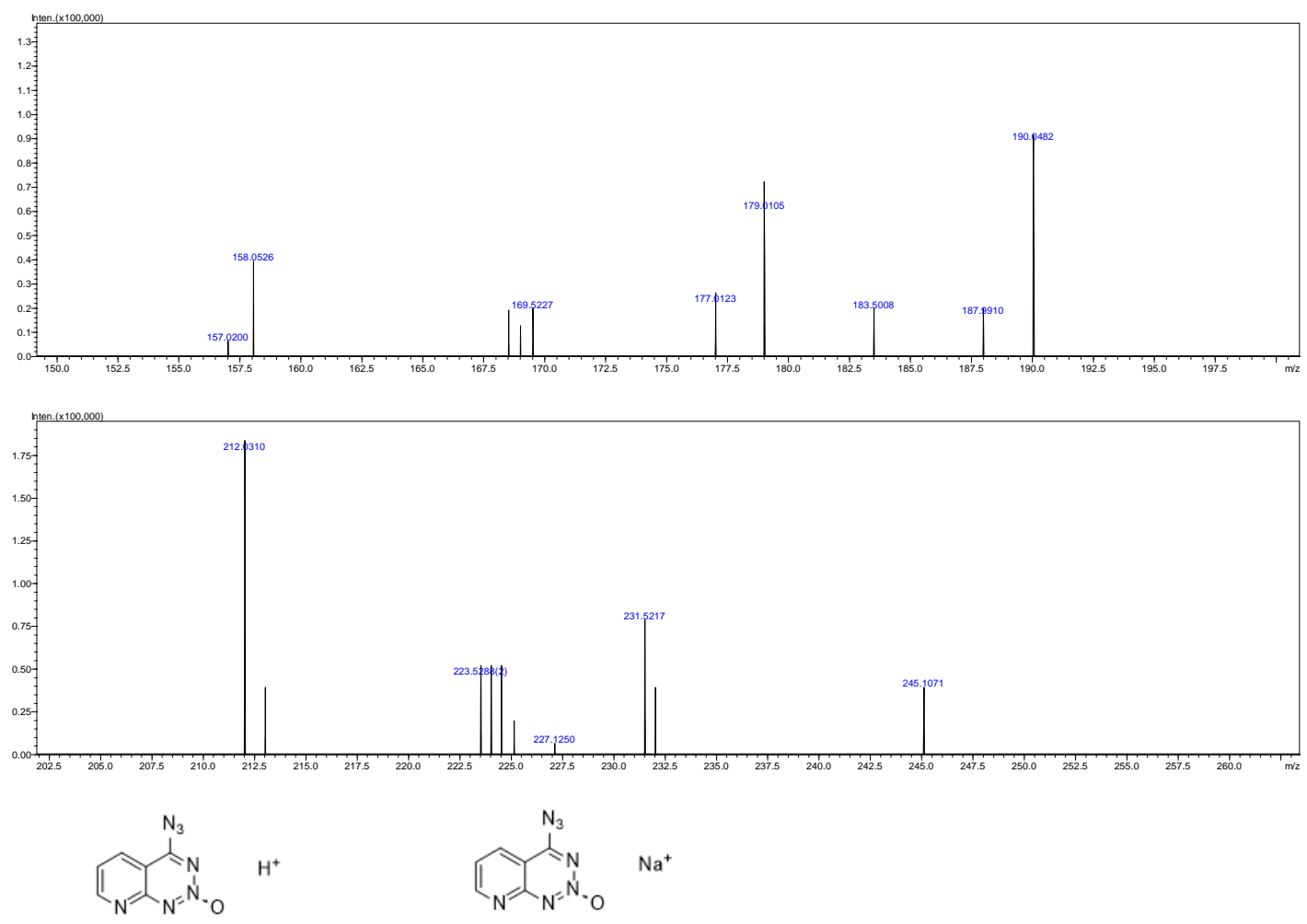

\begin{tabular}{|c|c|c|c|}
\hline Analysis & $=x$ & Analysis & $=x$ \\
\hline$\sqrt{\checkmark}$ Eormula: & $\mathrm{C}_{6} \mathrm{H}_{4} \mathrm{~N}_{7} \mathrm{O}^{+}$ & $\sqrt{\nabla}$ Eormula: & $\mathrm{C}_{6} \mathrm{H}_{3} \mathrm{~N}_{7} \mathrm{NaO}^{+}$ \\
\hline$\sqrt{\checkmark}$ Exact Mass: & 190.0472 & $\sqrt{ }$ Exact Mass: & Decimals: $\sqrt{4}-$ \\
\hline 『 Mol. wht:: & 190.1455 & $\sqrt{\nabla} \mathrm{Mol}$. Wt.: & 212.1272 \\
\hline$\sqrt{\nabla} \mathrm{m} / \mathrm{z}:$ & $\begin{array}{l}190.0472 \\
(100.0 \%), \\
191.0505(6.5 \%),\end{array}$ & $\sqrt{ } \mathrm{m} / \mathrm{z}:$ & $\begin{array}{l}212.0291 \\
(100.0 \%) \\
213.0325(6.5 \%)\end{array}$ \\
\hline $\begin{array}{l}\text { 『 Elem. Anal.: } \\
\text { Paste } \\
\end{array}$ & $\begin{array}{l}\text { C, } 37.90 ; \mathrm{H}, 2.12 ; \mathrm{N}, 51.57 \\
0,8.41\end{array}$ & $\begin{array}{l}\text { V Elem. Anal.: } \\
\text { Paste } \\
\end{array}$ & $\begin{array}{l}\mathrm{C}, 33.97 ; \mathrm{H}, 1.43 ; \mathrm{N}, 46.22 ; \\
\mathrm{Na}, 10.84 ; \mathrm{O}, 7.54\end{array}$ \\
\hline
\end{tabular}

Figure S125. HRMS(ESI+) of 3b.

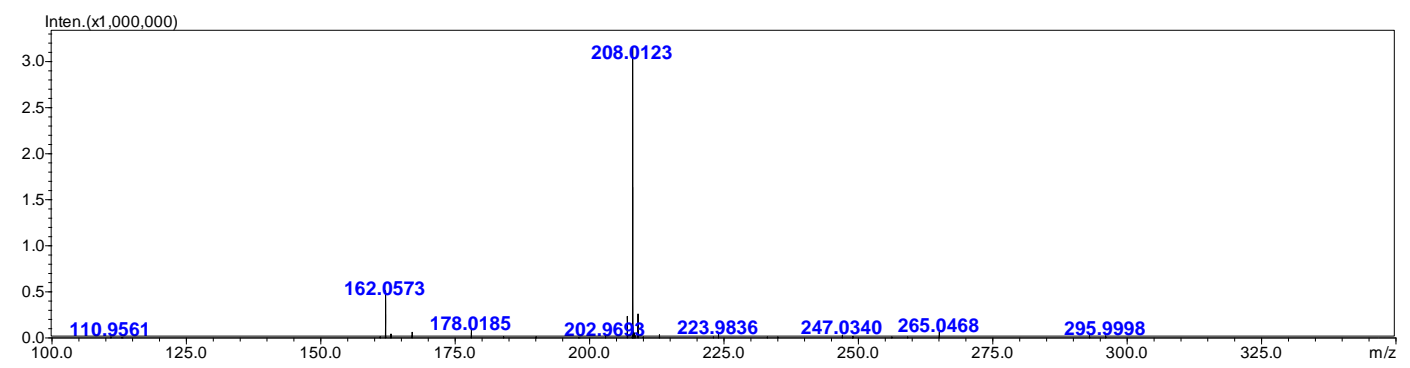




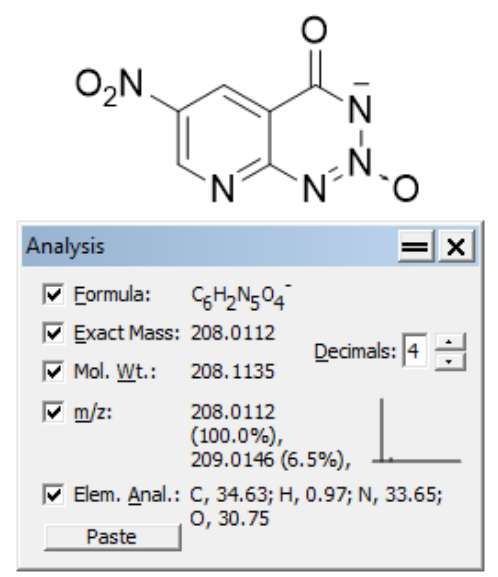

Figure S126. HRMS(ESI-) of 3b-1.
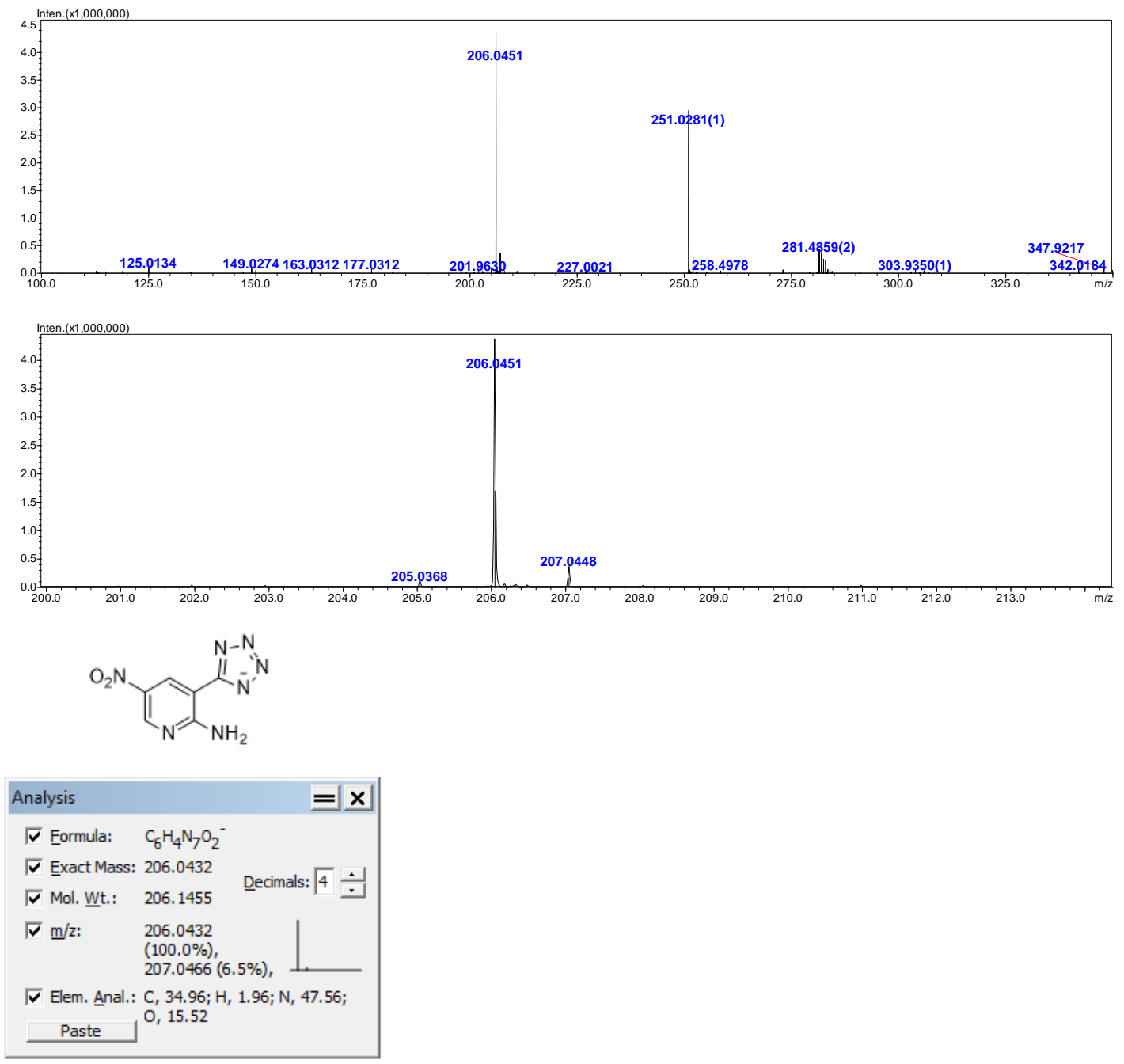

Figure S127. HRMS(ESI-) of $\mathbf{3 b - 2}$. 


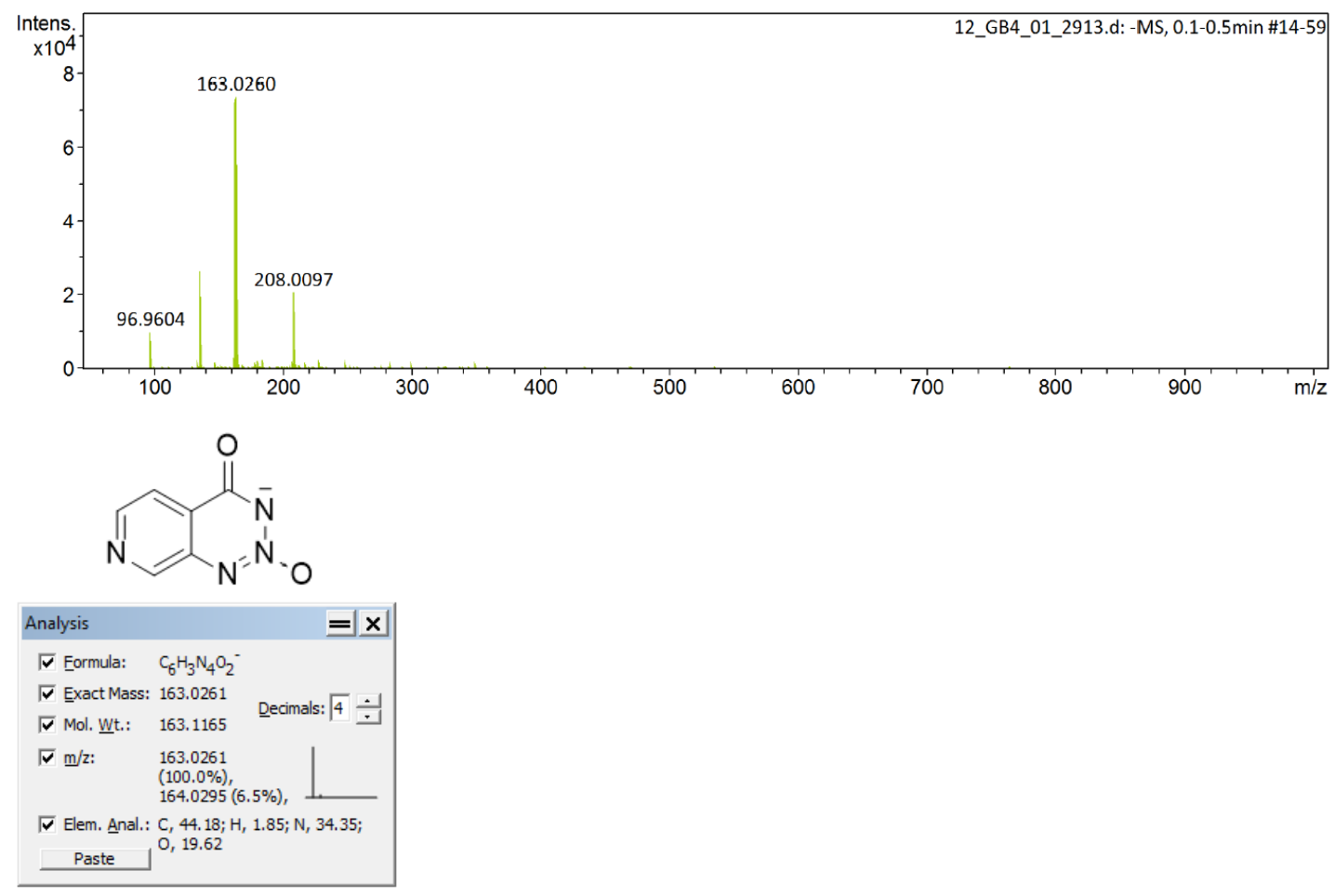

Figure S128. HRMS(ESI-) of 3c-1.
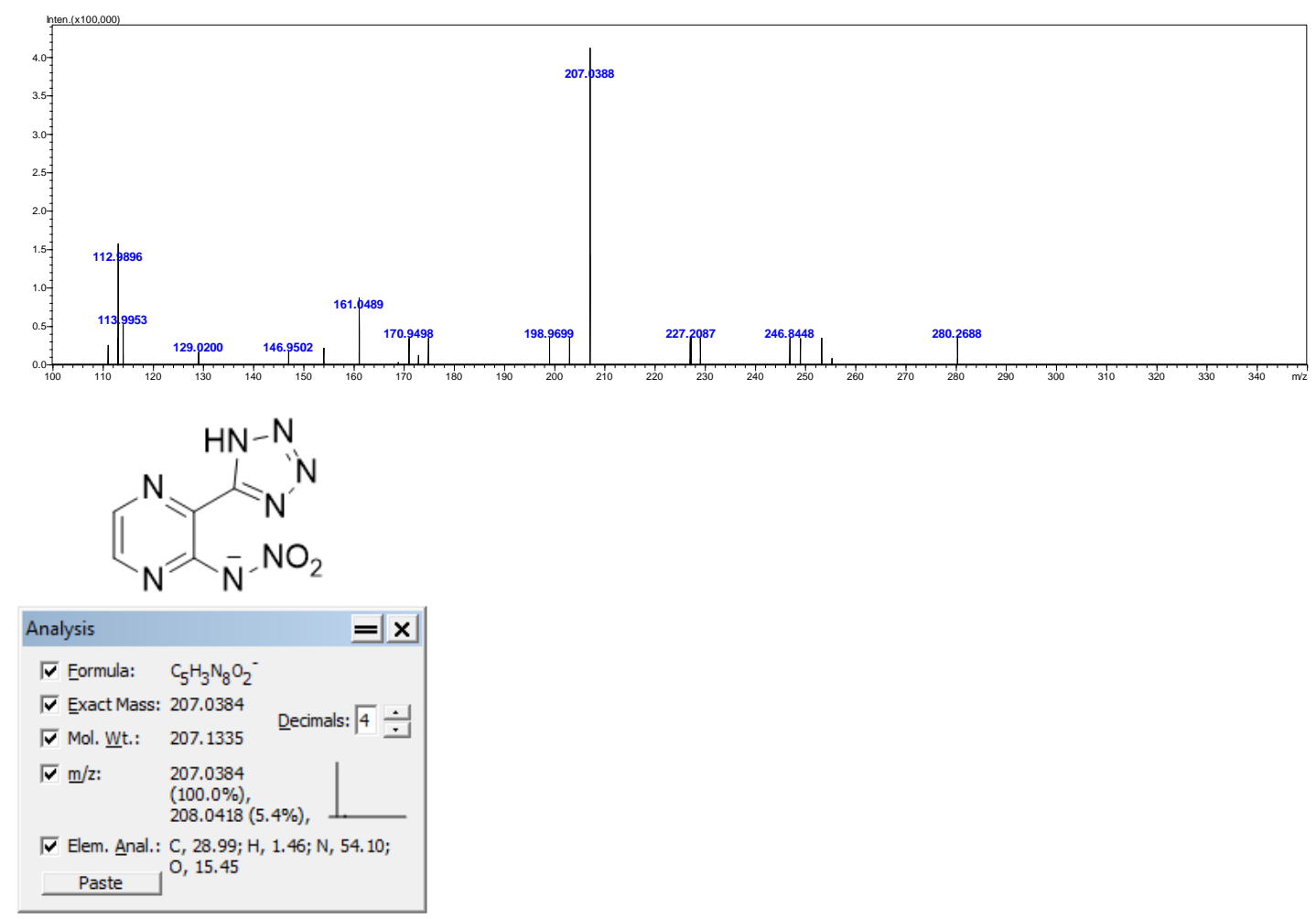

Figure S129. HRMS(ESI-) of 3f-1. 

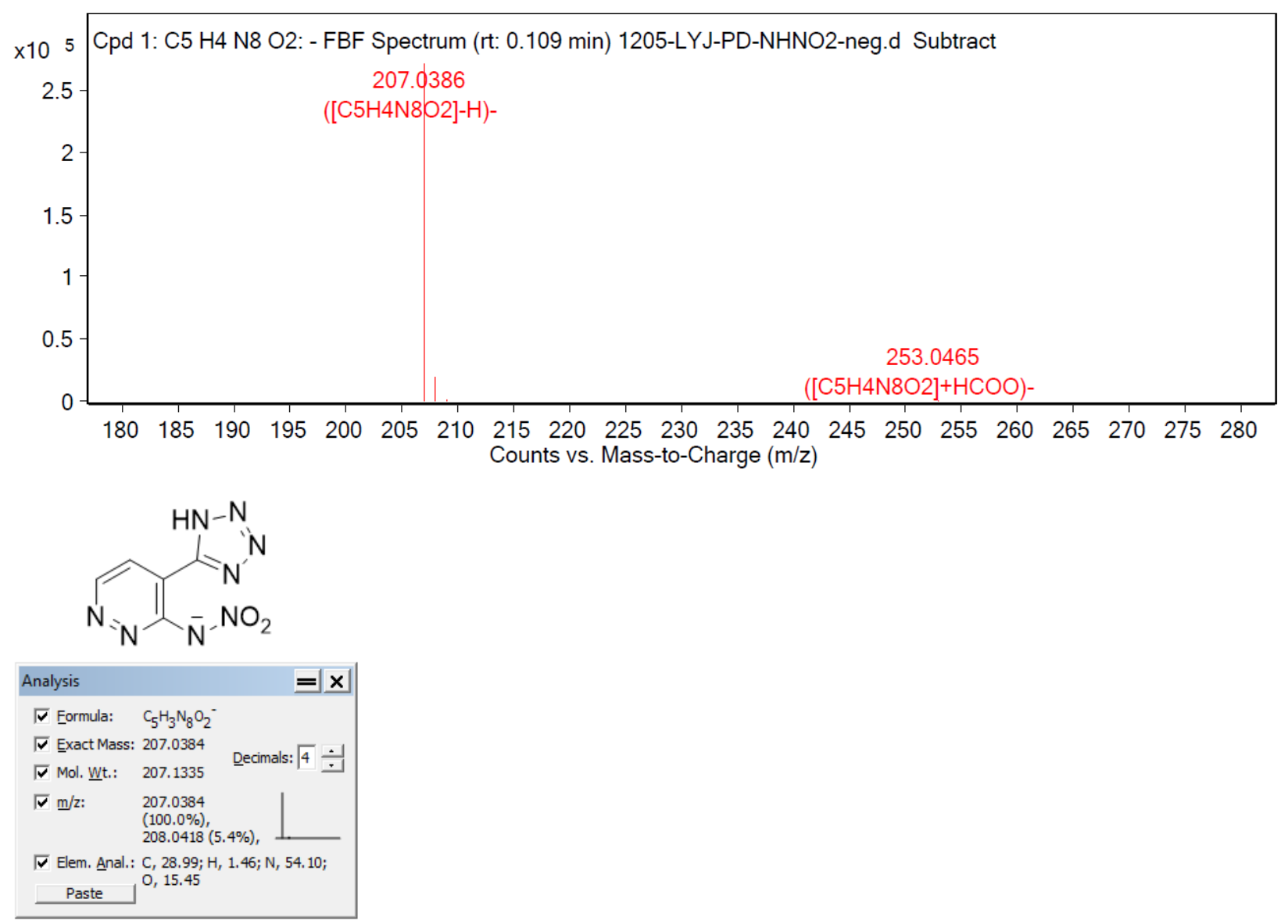

Figure S130. HRMS(ESI-) of 3g-1.
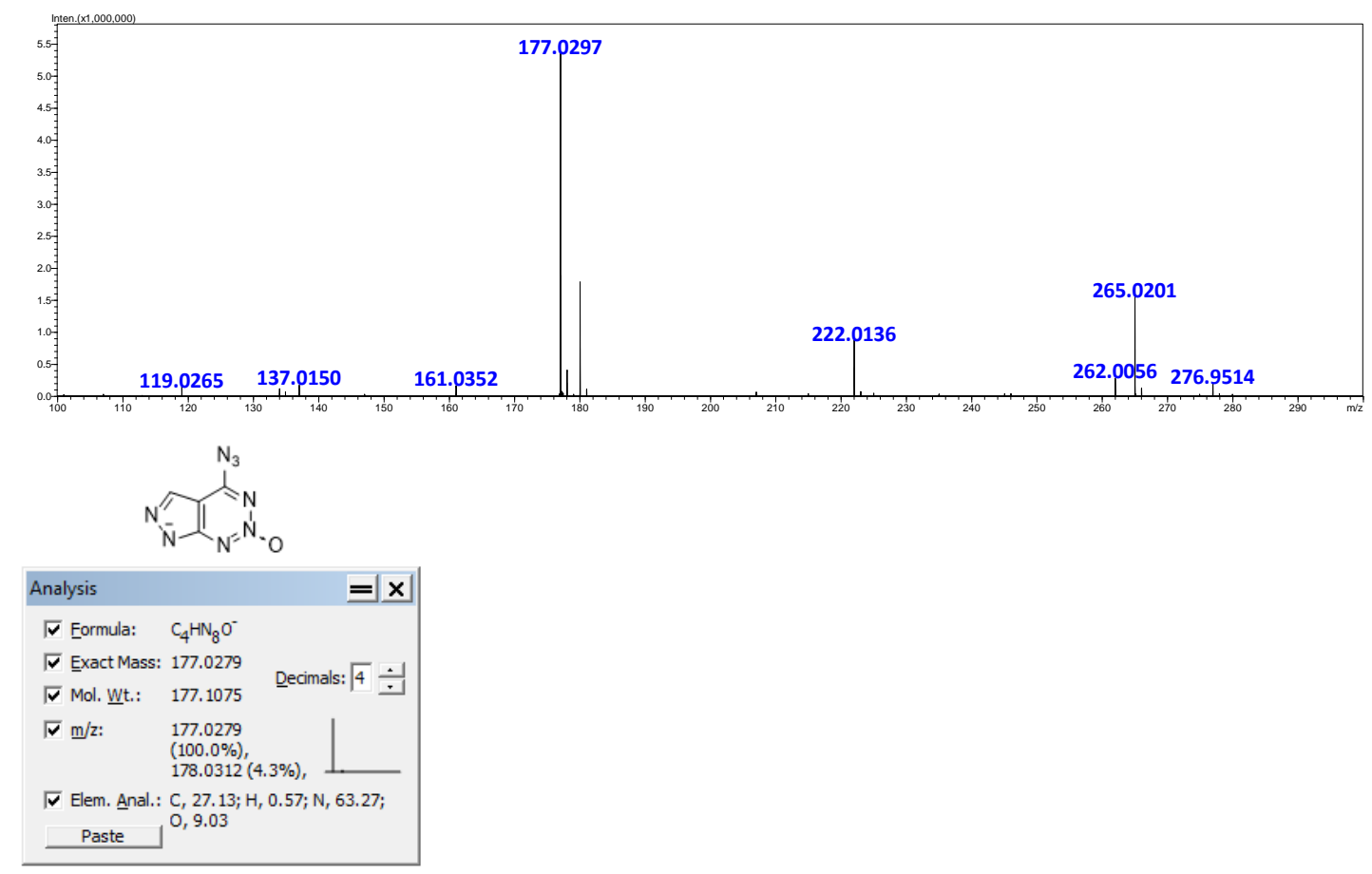
Figure S131. HRMS(ESI-) of 3i.
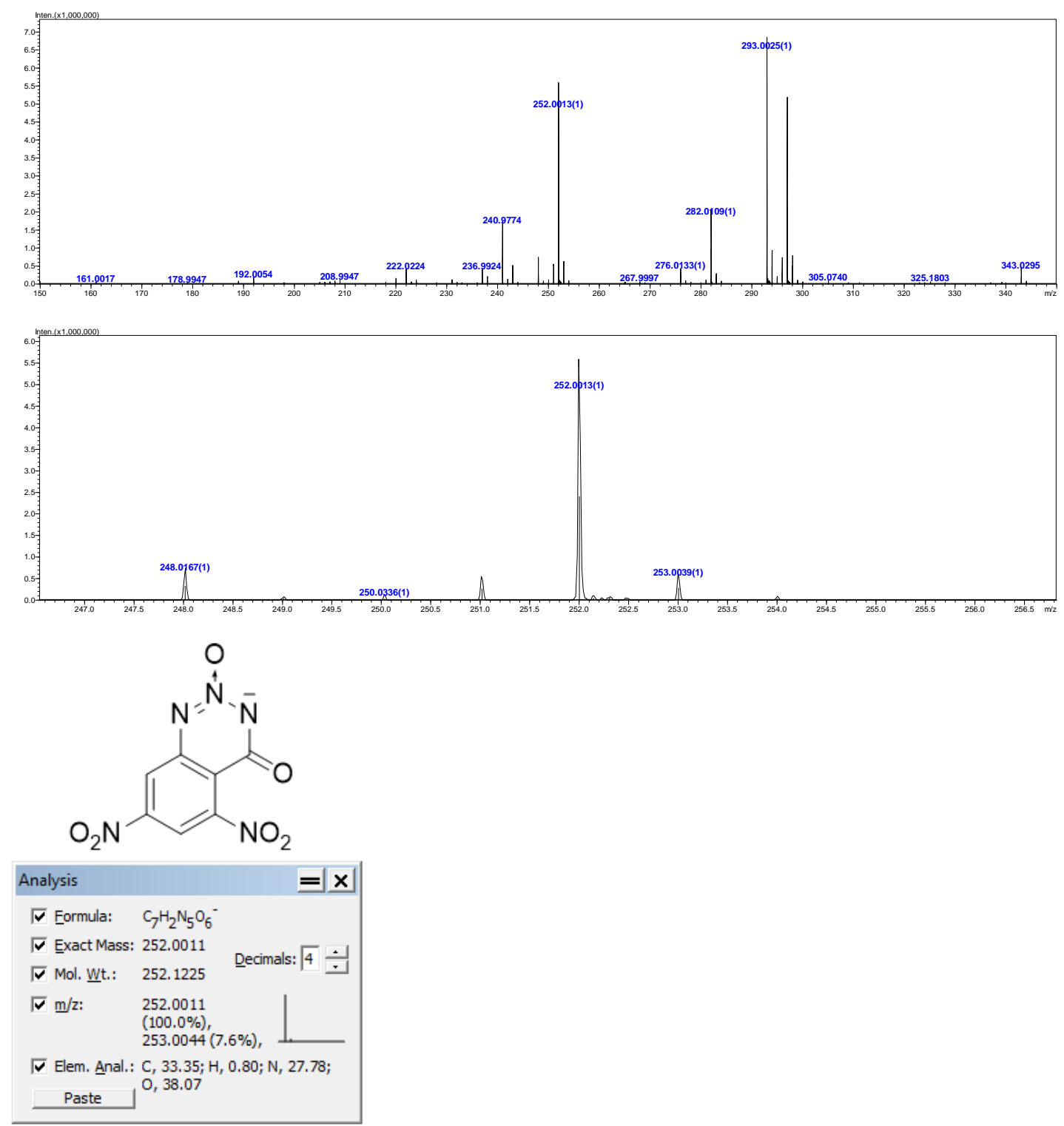

Figure S132. HRMS(ESI-) of 3I-1.

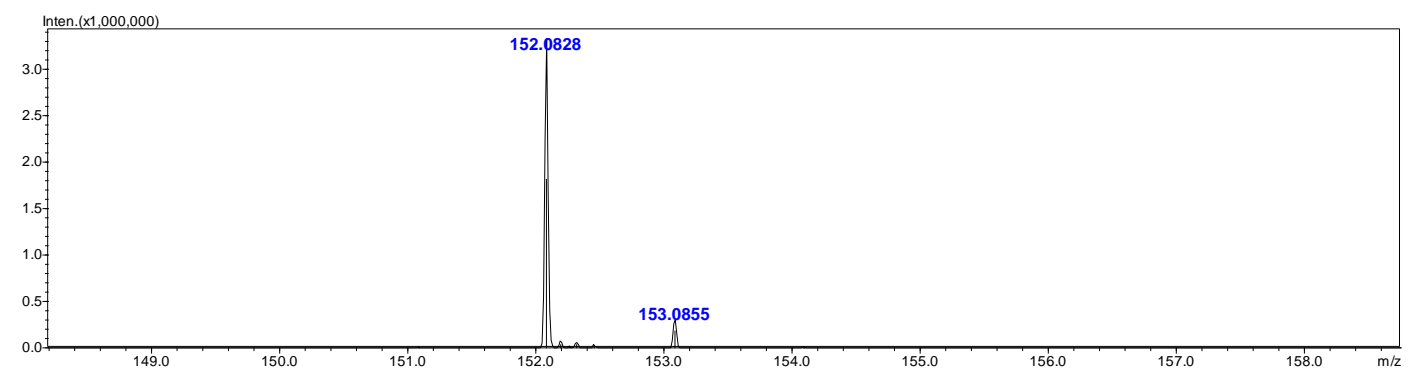




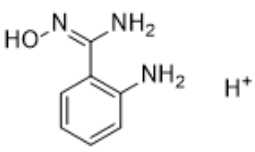

\begin{tabular}{|c|c|}
\hline Analysis & $=x$ \\
\hline$\sqrt{\checkmark}$ Eormula: & $\mathrm{C}_{7} \mathrm{H}_{10} \mathrm{~N}_{3} \mathrm{O}^{+}$ \\
\hline$\sqrt{ }$ Exact Mass: & Decimals: $\sqrt{4}-$ \\
\hline$\sqrt{\nabla}$ Mol. wht.: & 152.1765 \\
\hline$\sqrt{V} \mathrm{~m} / \mathrm{z}:$ & $\begin{array}{l}152.0818 \\
(100.0 \%), \\
153.0852(7.6 \%),\end{array}$ \\
\hline $\begin{array}{c}\sqrt{\checkmark} \text { Elem. Anal.: } \\
\text { Paste }\end{array}$ & $\begin{array}{l}\text { C, } 55.25 ; \mathrm{H}, 6.62 ; \mathrm{N}, 27.61 \\
\mathrm{O}, 10.51\end{array}$ \\
\hline
\end{tabular}

Figure S133. HRMS(ESI+) of 4 a.
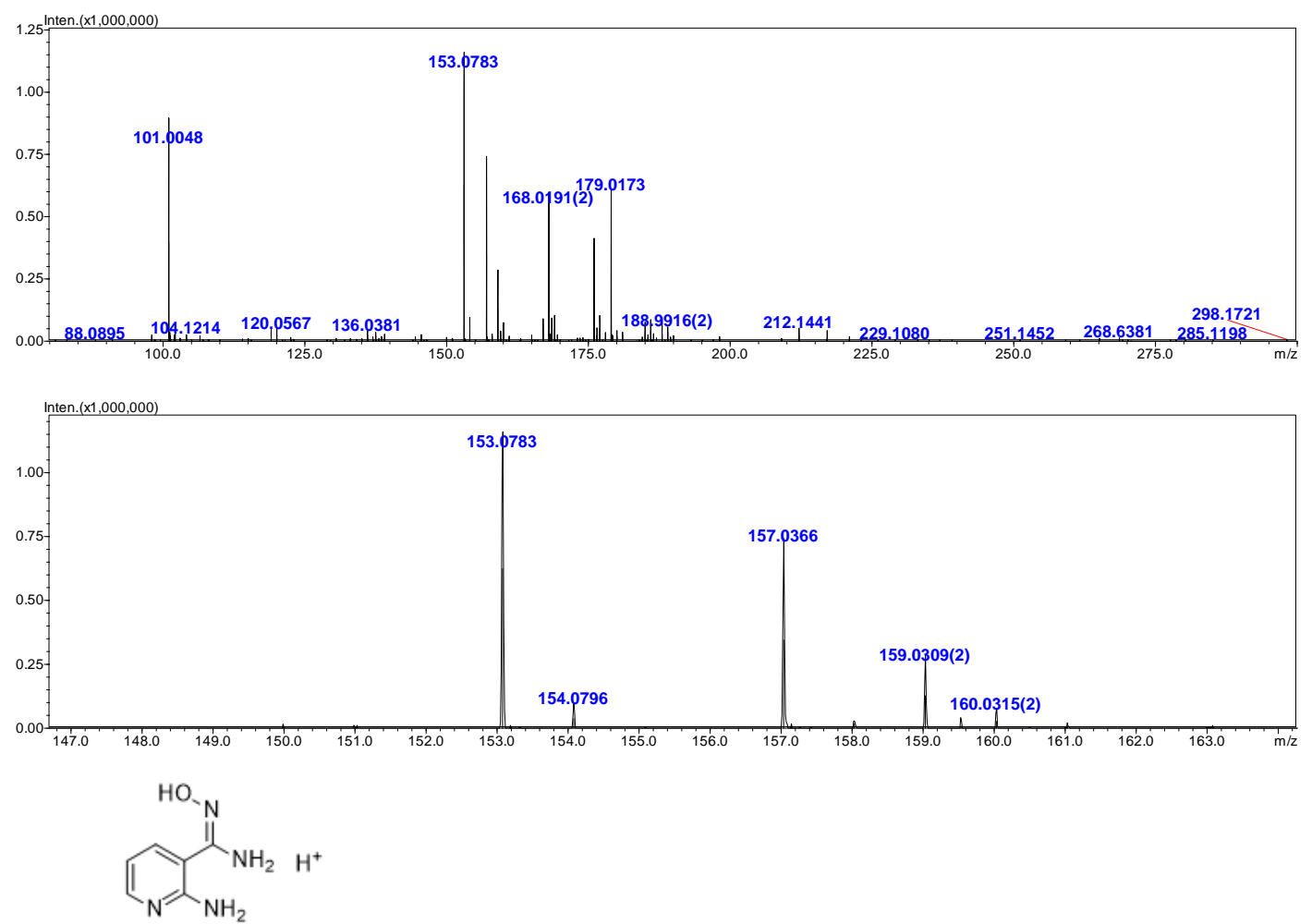

\begin{tabular}{|c|c|}
\hline Analysis & $=x$ \\
\hline$\sqrt{\checkmark}$ Eormula: & $\mathrm{C}_{6} \mathrm{H}_{9} \mathrm{~N}_{4} \mathrm{O}^{+}$ \\
\hline$\sqrt{\checkmark}$ Exact Mass: & 153.0771 \\
\hline$\sqrt{\checkmark}$ Mol. $\underline{\text { Wt.: }}$ & 153.1645 \\
\hline$\sqrt{ } \mathrm{m} / \mathrm{z}:$ & $\begin{array}{l}153.0771 \\
(100.0 \%), \\
154.0804(6.5 \%),\end{array}$ \\
\hline $\begin{array}{l}\sqrt{\checkmark} \text { Elem. Anal.: } \\
\text { Paste } \\
\end{array}$ & $\begin{array}{l}\text { C, } 47.05 ; \mathrm{H}, 5.92 ; \mathrm{N}, 36.58 \\
\text { O, } 10.45\end{array}$ \\
\hline
\end{tabular}

Figure S134. HRMS(ESI+) of 4b. 

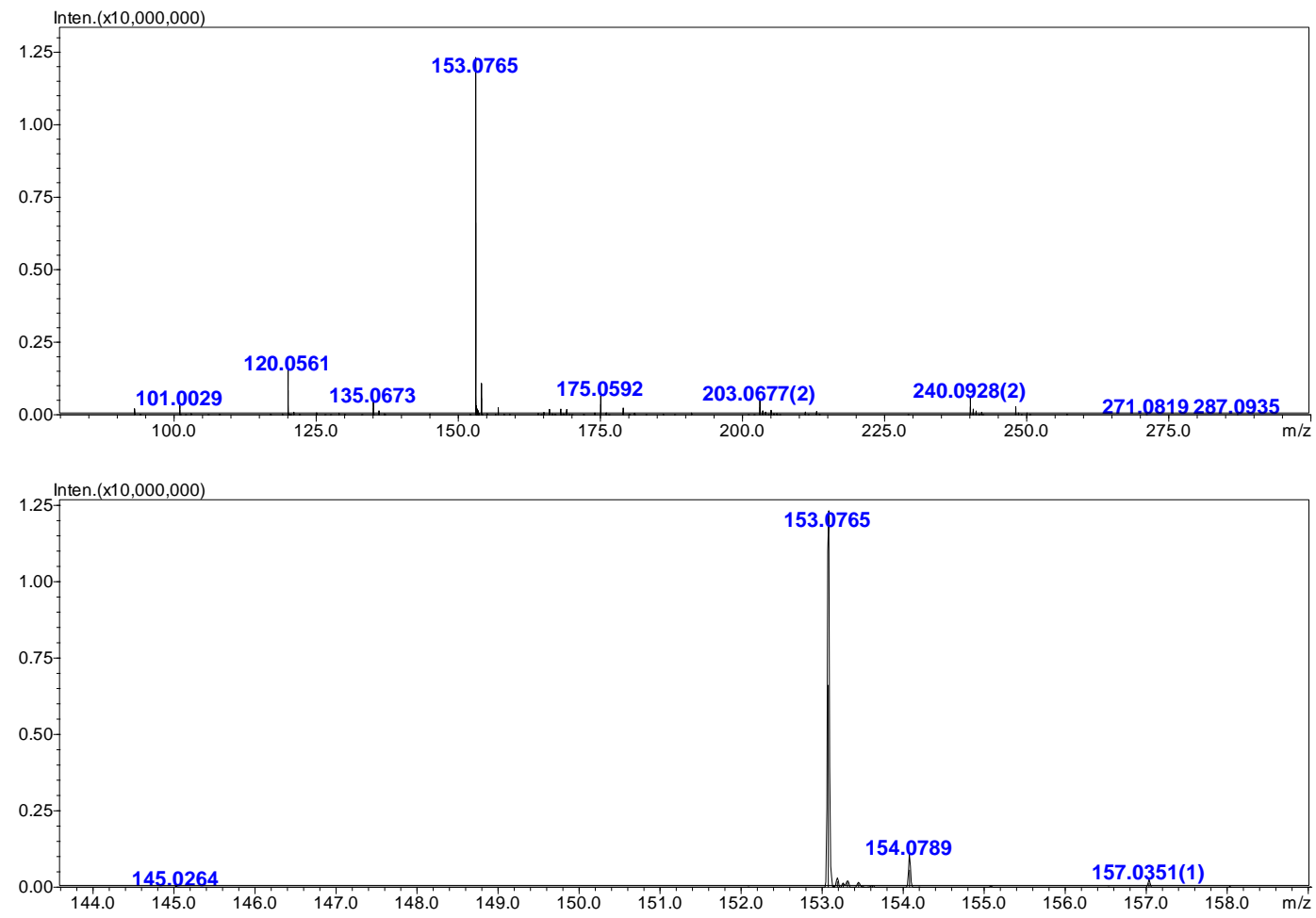

(N)

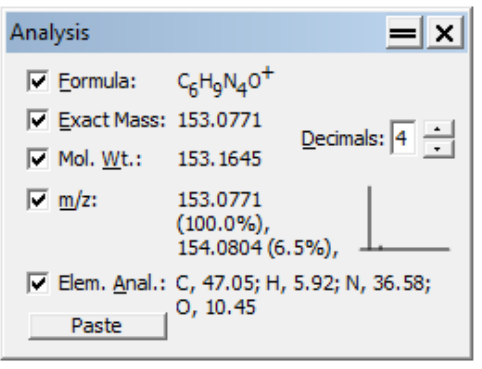

Figure S135. HRMS(ESI+) of 4c.

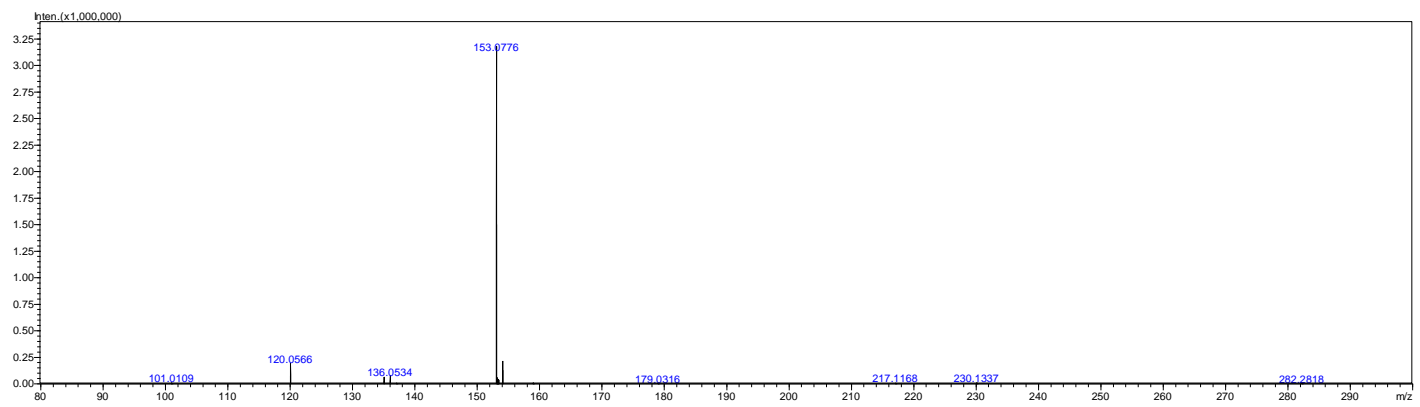




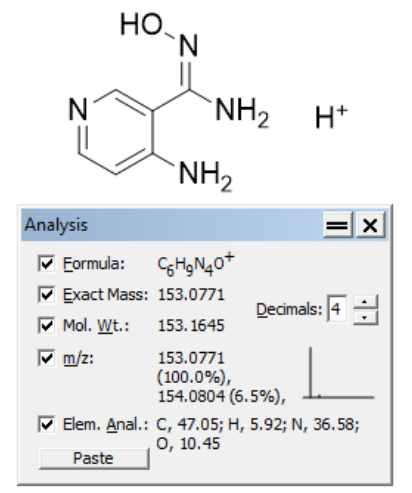

Figure S92. HRMS(ESI+) of $\mathbf{4 d}$.
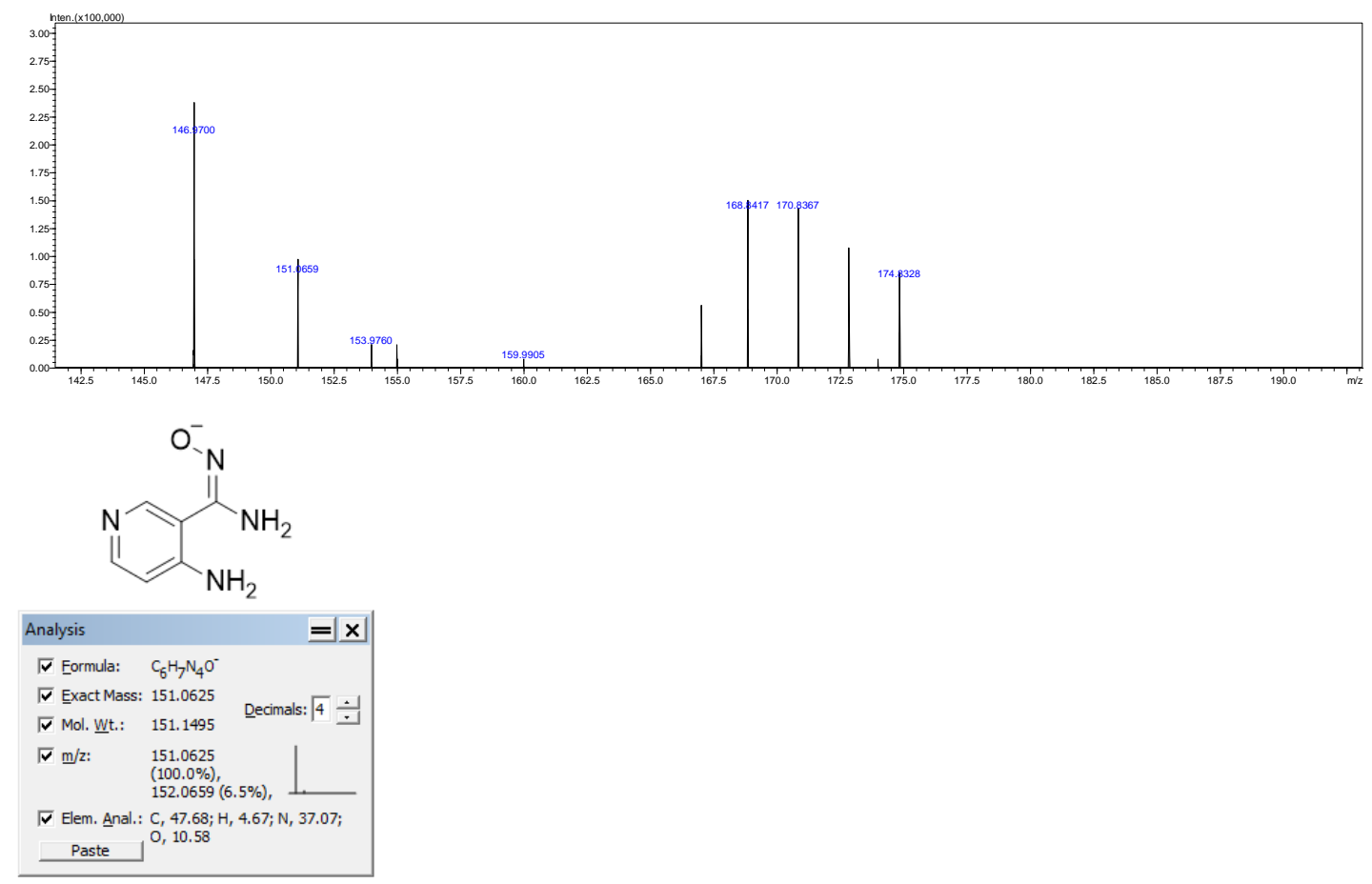

Figure S136. HRMS(ESI-) of 4d.

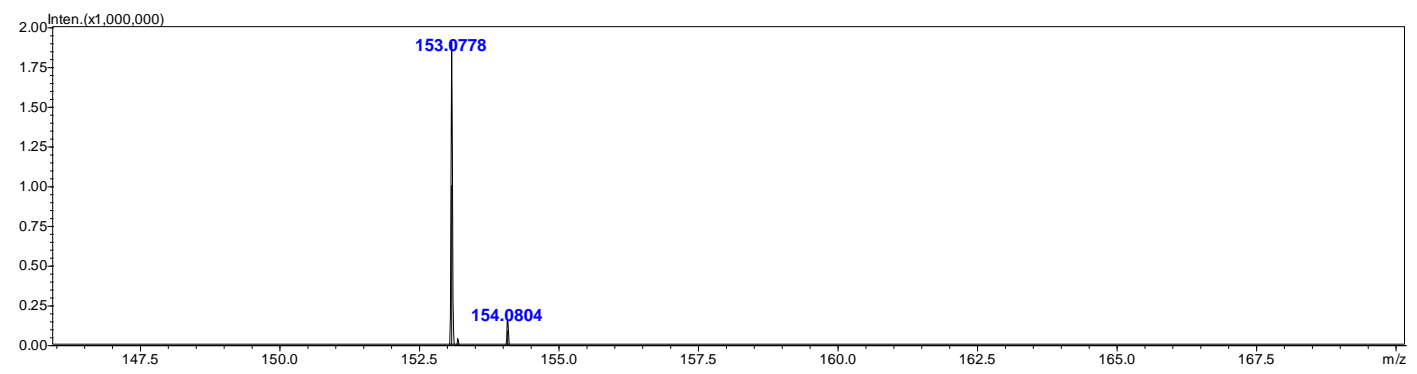


<smiles>N/C(=N/O)c1ncccc1N</smiles>

\begin{tabular}{|c|c|}
\hline Analysis & $=x$ \\
\hline$\sqrt{\checkmark}$ Eormula: & $\mathrm{C}_{6} \mathrm{H}_{9} \mathrm{~N}_{4} \mathrm{O}^{+}$ \\
\hline$\sqrt{\nabla}$ Exact Mass: & 153.0771 \\
\hline$\sqrt{\nabla}$ Mol. wht: & 153.1645 \\
\hline$\sqrt{\nabla} \mathrm{m} / \mathrm{z}:$ & $\begin{array}{l}153.0771 \\
(100.0 \%), \\
154.0804(6.5 \%),\end{array}$ \\
\hline $\begin{array}{l}\text { V Elem. Anal.: } \\
\text { Paste }\end{array}$ & $\begin{array}{l}\text { C, } 47.05 ; \mathrm{H}, 5.92 ; \mathrm{N}, 36.58 \\
\mathrm{O}, 10.45\end{array}$ \\
\hline
\end{tabular}

\section{Figure S137. HRMS(ESI+) of 4e.}

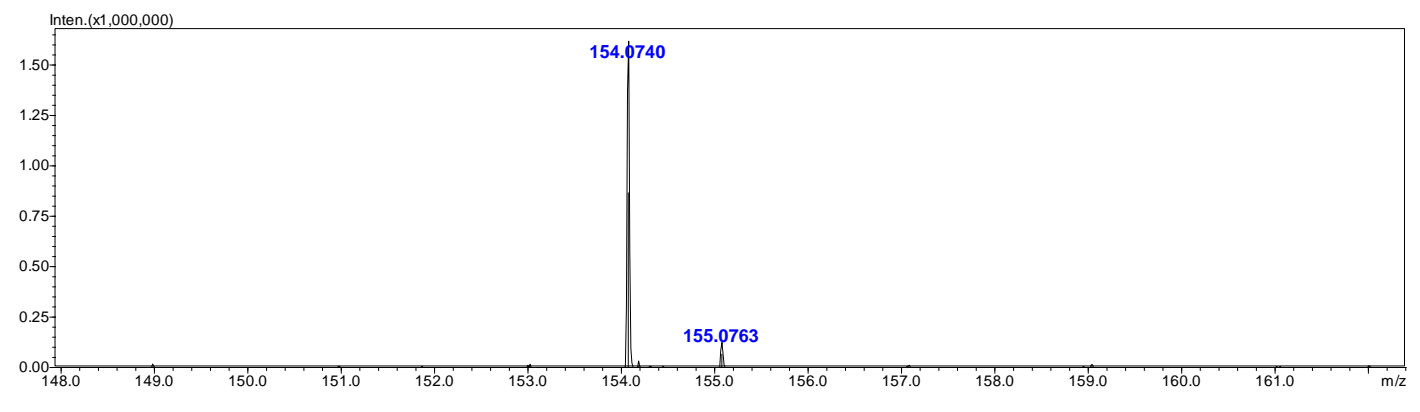<smiles>N/C(=N/O)c1nccnc1N</smiles>

\begin{tabular}{|c|c|c|}
\hline Analysis & $=$ & $x$ \\
\hline$\sqrt{\checkmark}$ Eormula: & \multirow{4}{*}{ Decimals: $\sqrt{4}$} & \multirow{4}{*}{$\div$} \\
\hline$\sqrt{\checkmark}$ Exact Mass: & & \\
\hline 『 Mol. $\underline{\text { Wt.: }}$ & & \\
\hline$\sqrt{ } \mathrm{m} / \mathrm{z}:$ & & \\
\hline $\begin{array}{l}\sqrt{\checkmark} \text { Elem. Anal.: } \\
\text { Paste } \\
\end{array}$ & $\begin{array}{l}\mathrm{C}, 38.96 ; \mathrm{H}, 5.23 ; \mathrm{N}, 45.43 \\
\mathrm{O}, 10.38\end{array}$ & \\
\hline
\end{tabular}

Figure S138. HRMS(ESI+) of $\mathbf{4 f}$.

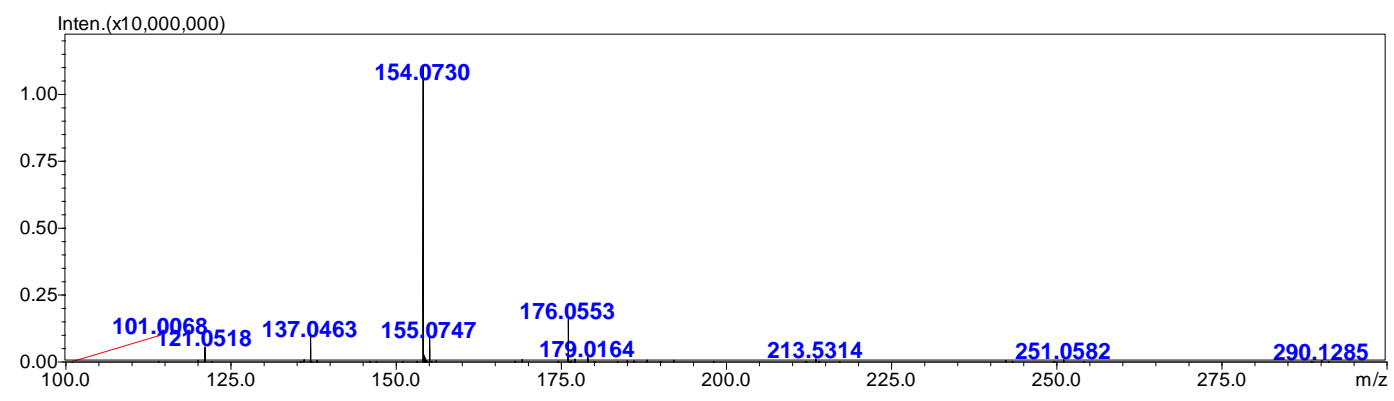



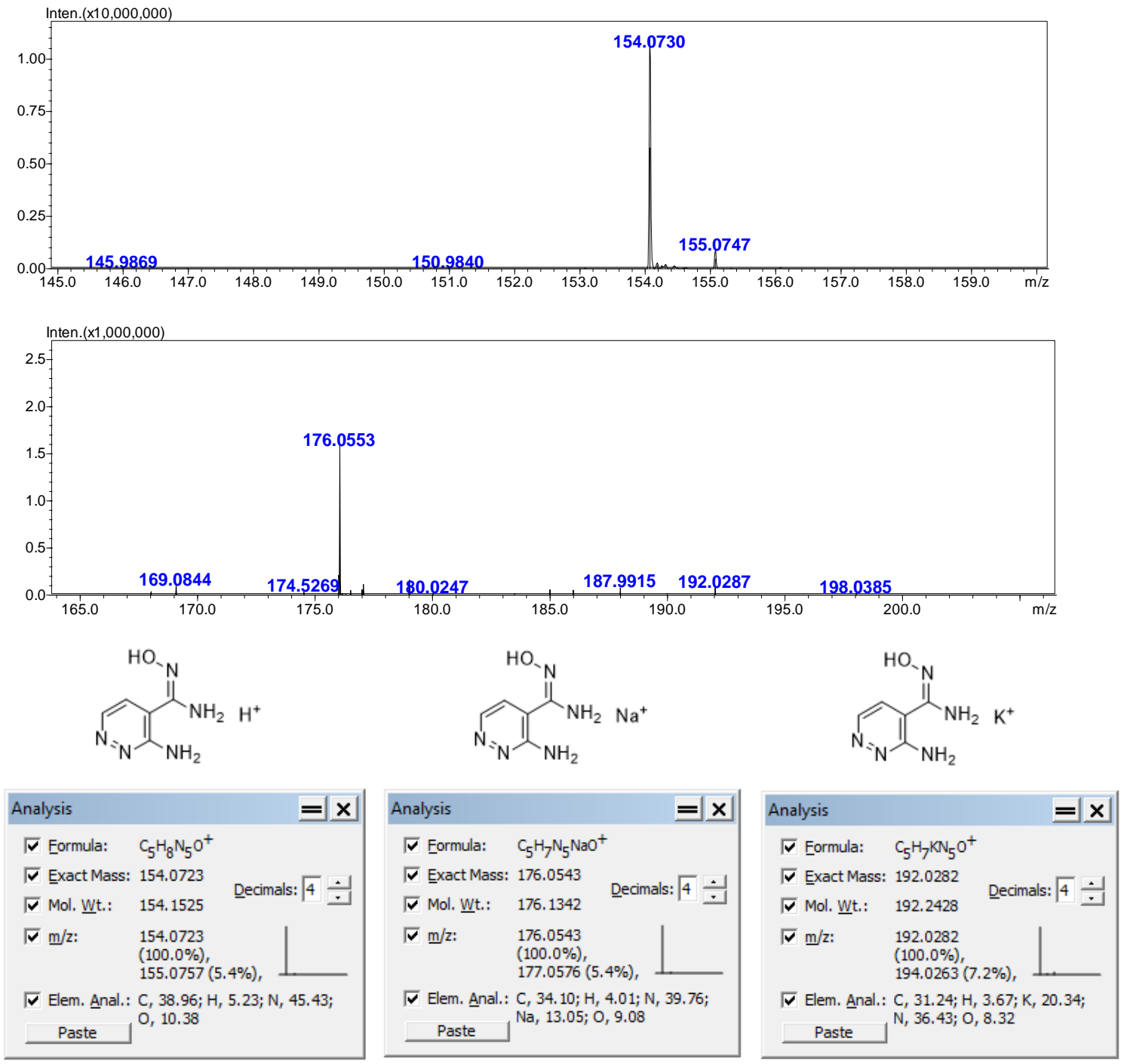

Figure S139. HRMS(ESI+) of 4g.

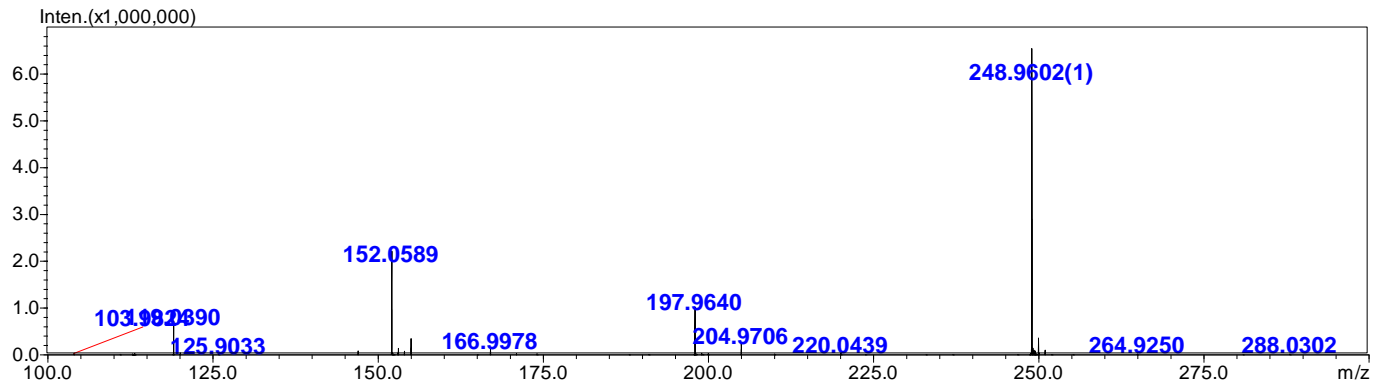




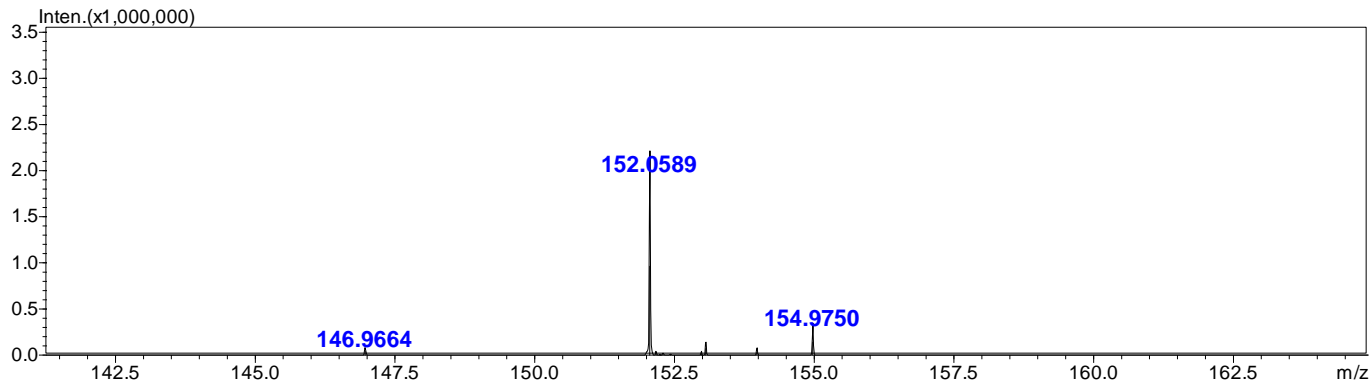<smiles>N/C(=N/[O-])c1ccnnc1N</smiles>

\begin{tabular}{|c|c|}
\hline Analysis & $=x$ \\
\hline 『 Eormula: & $\mathrm{C}_{5} \mathrm{H}_{6} \mathrm{~N}_{5} \mathrm{O}^{-}$ \\
\hline V Exact Mass: & 152.0578 \\
\hline 『 Mol. wht: & 152.1375 \\
\hline$\sqrt{\nabla} \mathrm{m} / \mathrm{z}:$ & $\begin{array}{l}152.0578 \\
(100.0 \%), \\
153.0611(5.4 \%),\end{array}$ \\
\hline $\begin{array}{l}\text { V Elem. Anal.: } \\
\text { Paste }\end{array}$ & $\begin{array}{l}\mathrm{C}, 39.47 ; \mathrm{H}, 3.98 ; \mathrm{N}, 46.03 \\
0,10.52\end{array}$ \\
\hline
\end{tabular}

Figure S140. HRMS(ESI-) of 4g.
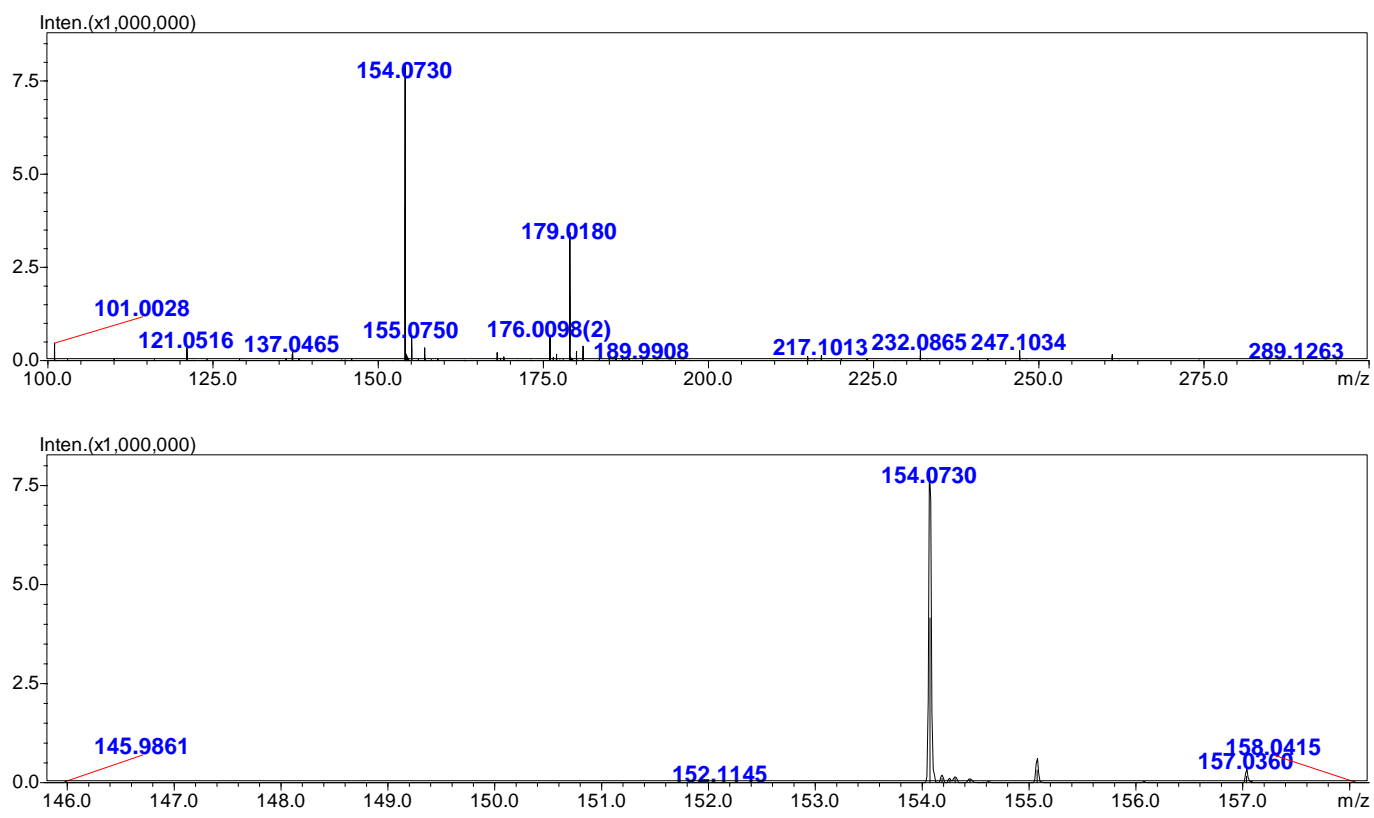


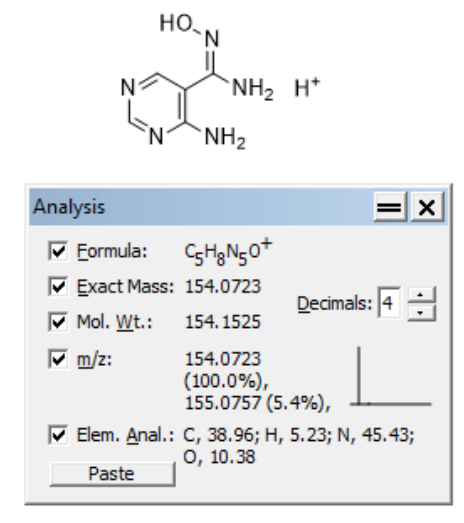

Figure S141. HRMS(ESI+) of $\mathbf{4 h}$.

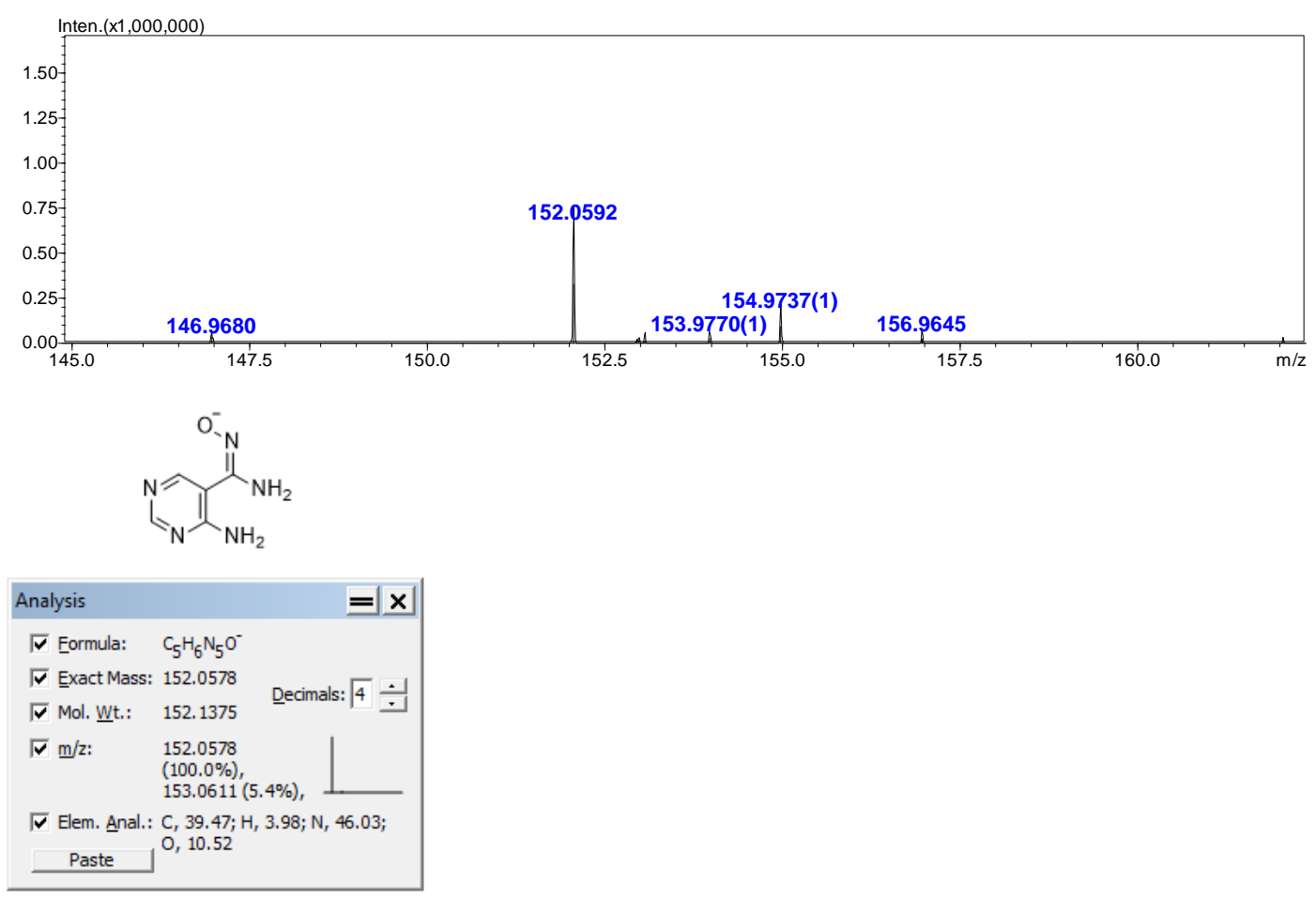

Figure S142. HRMS(ESI-) of $\mathbf{4 h}$.

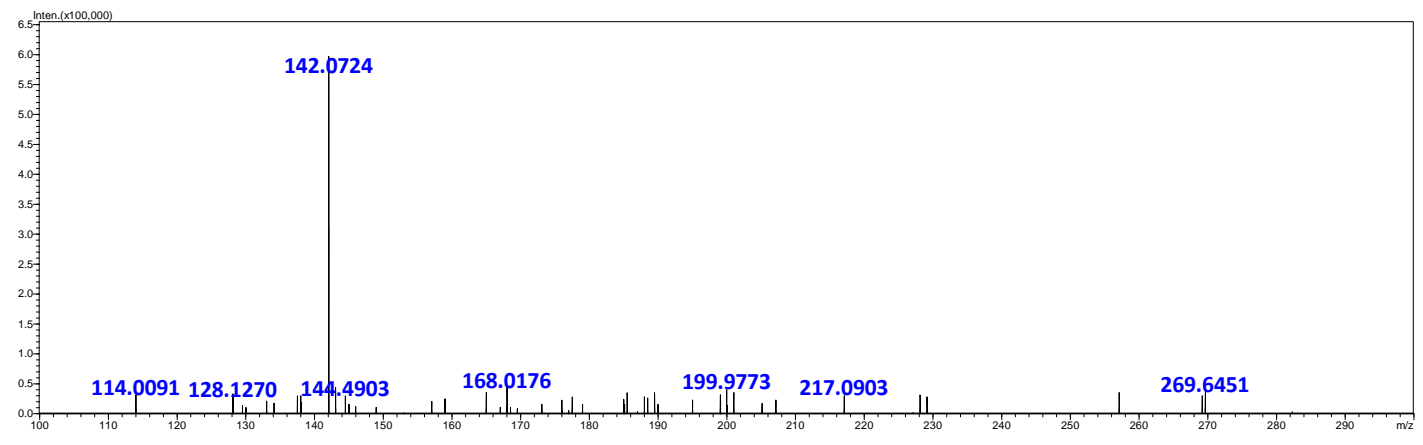




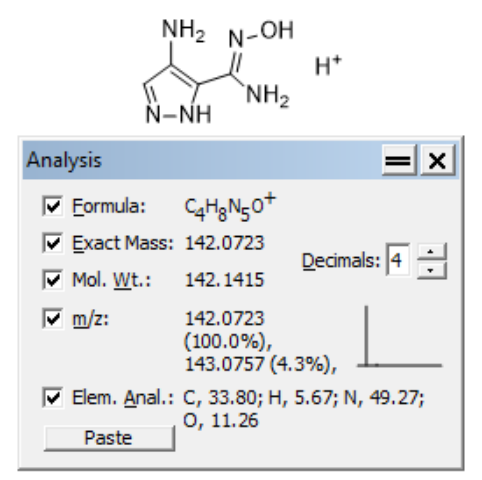

Figure S143. HRMS(ESI+) of $\mathbf{4 j}$.
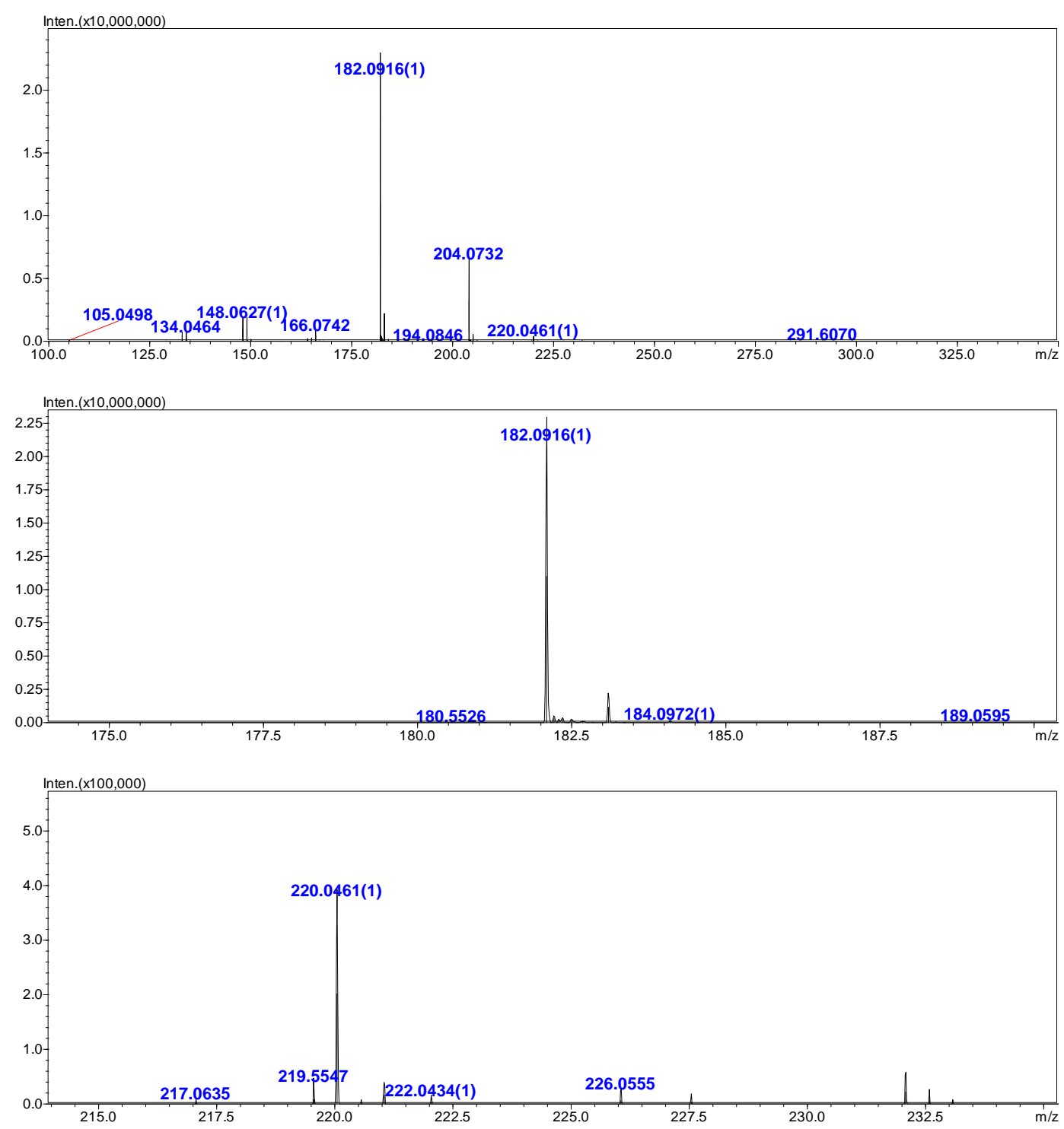
<smiles>COc1cccc(N)c1/C(N)=N/O</smiles>

\begin{tabular}{|c|c|}
\hline Analysis & $=x$ \\
\hline$\sqrt{\checkmark}$ Eormula: & $\mathrm{C}_{8} \mathrm{H}_{12} \mathrm{~N}_{3} \mathrm{O}_{2}^{+}$ \\
\hline$\sqrt{ }$ Exact Mass: & 182.0924 Decimals: $\sqrt{4}$ \\
\hline$\sqrt{\nabla}$ Mol. wt.: & 182.2025 \\
\hline$\sqrt{\nabla} \mathrm{m} / \mathrm{z}:$ & $\begin{array}{l}182.0924 \\
(100.0 \%) \\
183.0958(8.7 \%)\end{array}$ \\
\hline $\begin{array}{l}\text { F Elem. Anal.: } \\
\text { Paste } \\
\end{array}$ & $\begin{array}{l}\text { C, } 52.74 ; \mathrm{H}, 6.64 ; \mathrm{N}, 23.06 \\
\text { O, } 17.56\end{array}$ \\
\hline
\end{tabular}<smiles>COc1cccc(N)c1/C(N)=N/O</smiles>

\begin{tabular}{|c|c|}
\hline Analysis & $=x$ \\
\hline$\sqrt{\checkmark}$ Eormula: & $\mathrm{C}_{8} \mathrm{H}_{11} \mathrm{~N}_{3} \mathrm{NaO}_{2}^{+}$ \\
\hline$\sqrt{ }$ Exact Mass & 204.0743 Decimals: $\sqrt{4}+$ \\
\hline$\sqrt{\nabla}$ Mol. wht.: & 204.1842 \\
\hline$\sqrt{\nabla} \mathrm{m} / \mathrm{z}:$ & $\begin{array}{l}204.0743 \\
(100.0 \%), \\
205.0777(8.7 \%),\end{array}$ \\
\hline $\begin{array}{l}\text { VV Elem. Anal. } \\
\text { Paste }\end{array}$ & $\begin{array}{l}\mathrm{C}, 47.06 ; \mathrm{H}, 5.43 ; \mathrm{N}, 20.58 \\
\mathrm{Na}, 11.26 ; \mathrm{O}, 15.67\end{array}$ \\
\hline
\end{tabular}<smiles>COc1cccc(N)c1/C(N)=N/O</smiles>

\begin{tabular}{|c|c|}
\hline Analysis & $=x$ \\
\hline$\sqrt{V}$ Eormula: & $\mathrm{C}_{8} \mathrm{H}_{11} \mathrm{KN}_{3} \mathrm{O}_{2}{ }^{+}$ \\
\hline$\sqrt{\nabla}$ Exact Mass: & 220.0483 \\
\hline$\sqrt{\nabla}$ Mol. $\underline{w}$ t.: & 220.2928 \\
\hline$\sqrt{\nabla} \mathrm{m} / \mathrm{z}:$ & $\begin{array}{l}220.0483 \\
(100.0 \%), \\
221.0516(8.7 \%), \quad\end{array}$ \\
\hline $\begin{array}{l}\text { Г Elem. Anal.: } \\
\text { Paste } \\
\end{array}$ & $\begin{array}{l}\mathrm{C}, 43.62 ; \mathrm{H}, 5.03 ; \mathrm{K}, 17.75 ; \\
\mathrm{N}, 19.08 ; \mathrm{O}, 14.53\end{array}$ \\
\hline
\end{tabular}

\section{Figure S144. HRMS(ESI+) of 4k.}
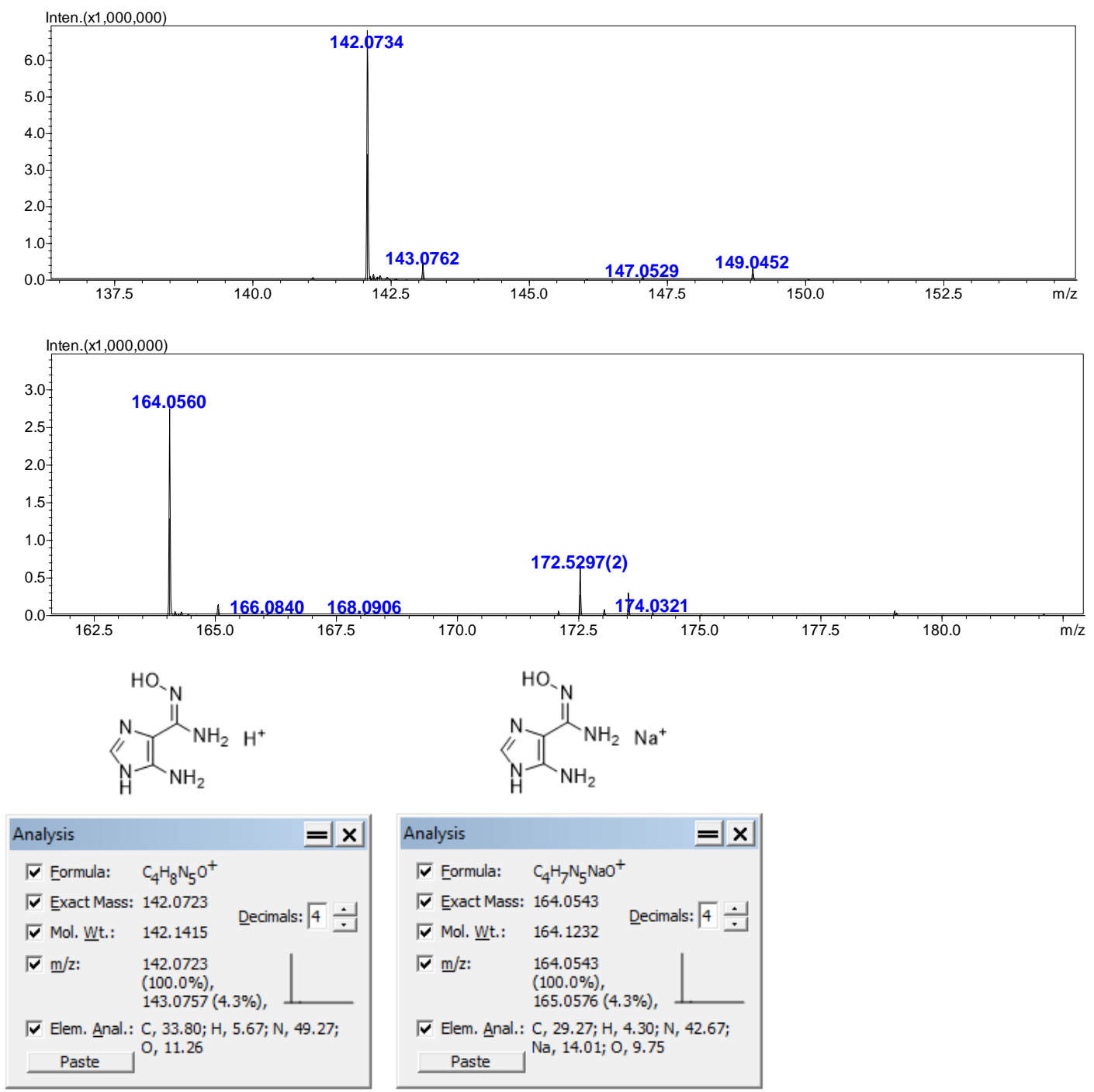

Figure S145. HRMS(ESI+) of 4m. 

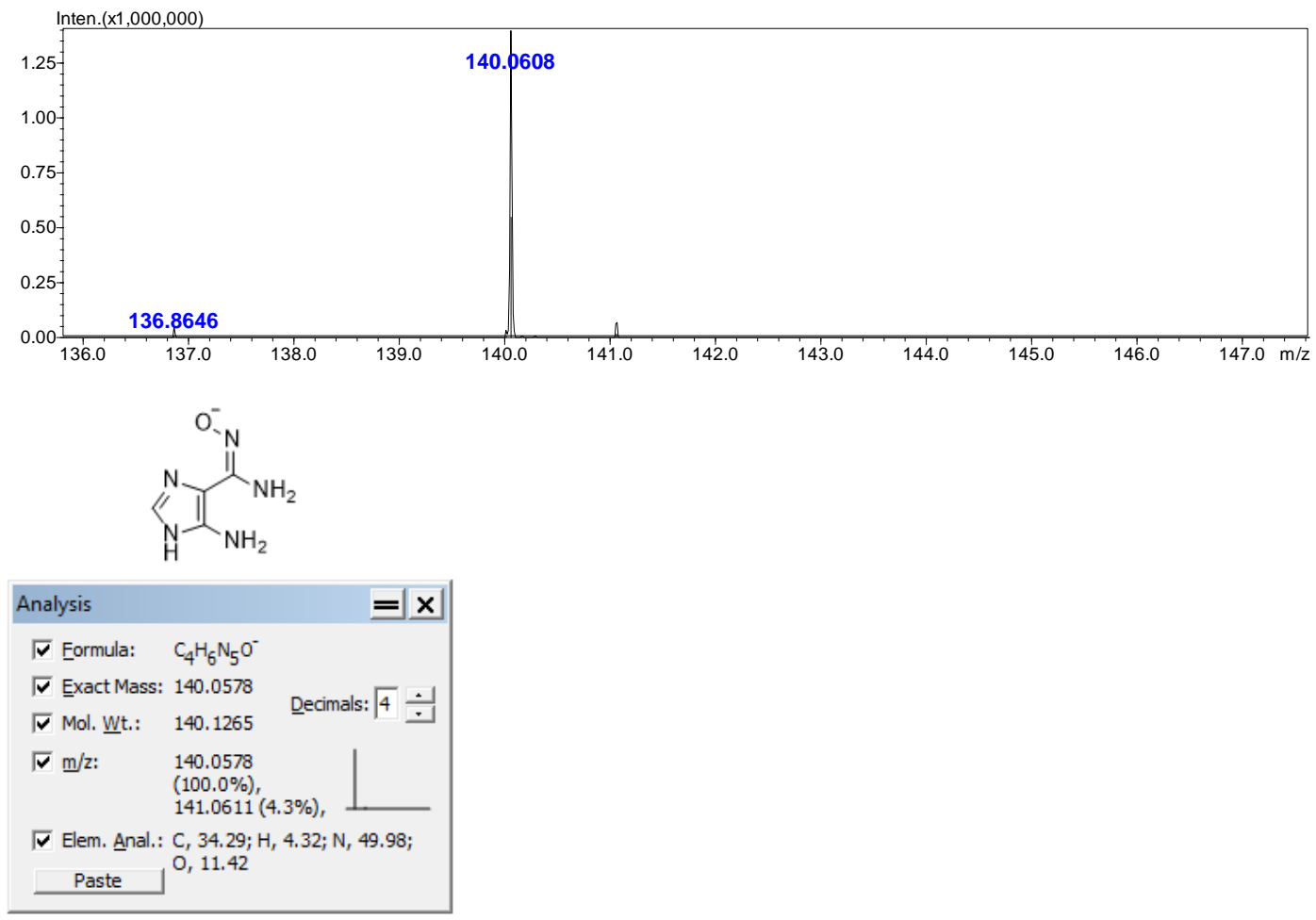

Figure S146. HRMS(ESI-) of $\mathbf{4 m}$.
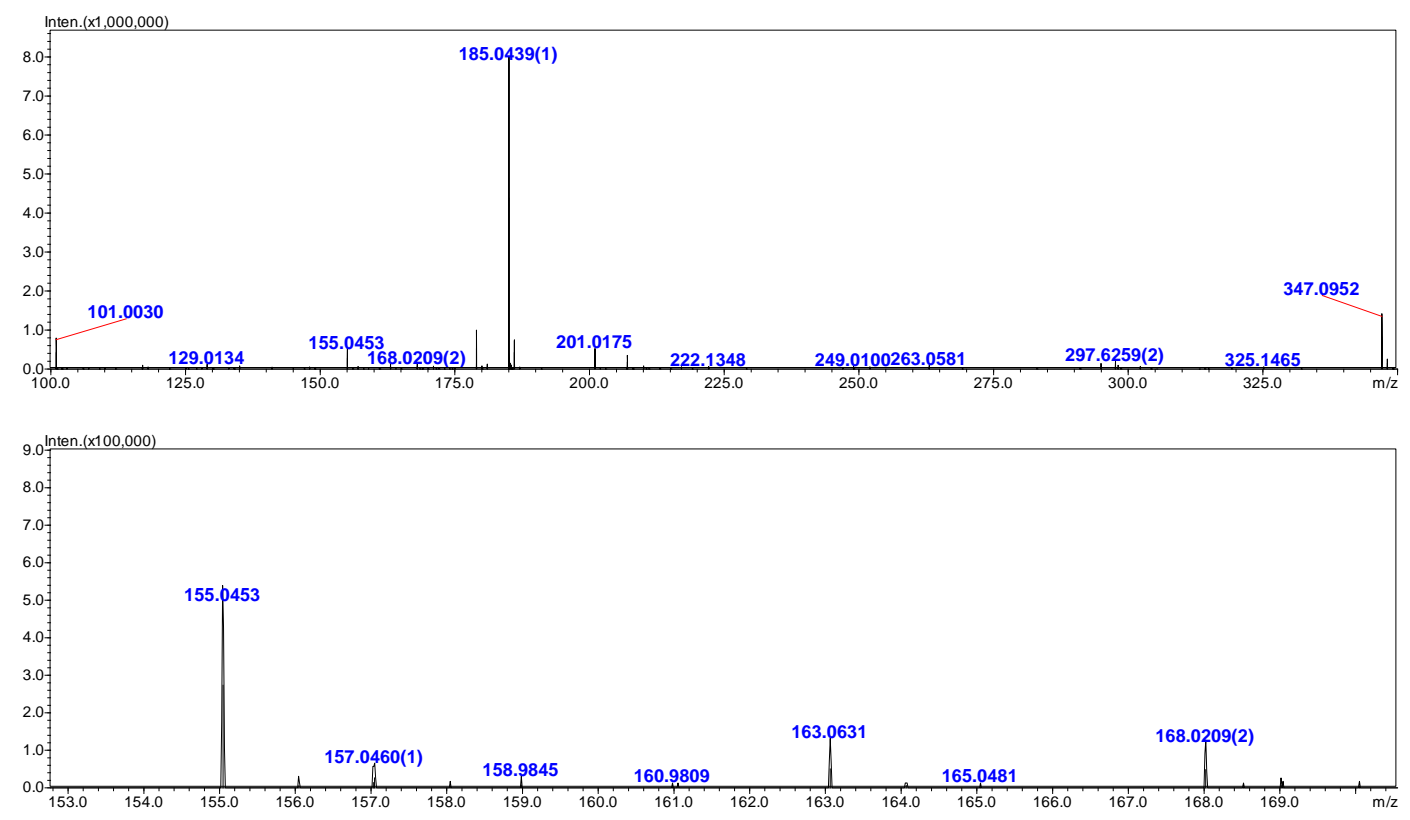

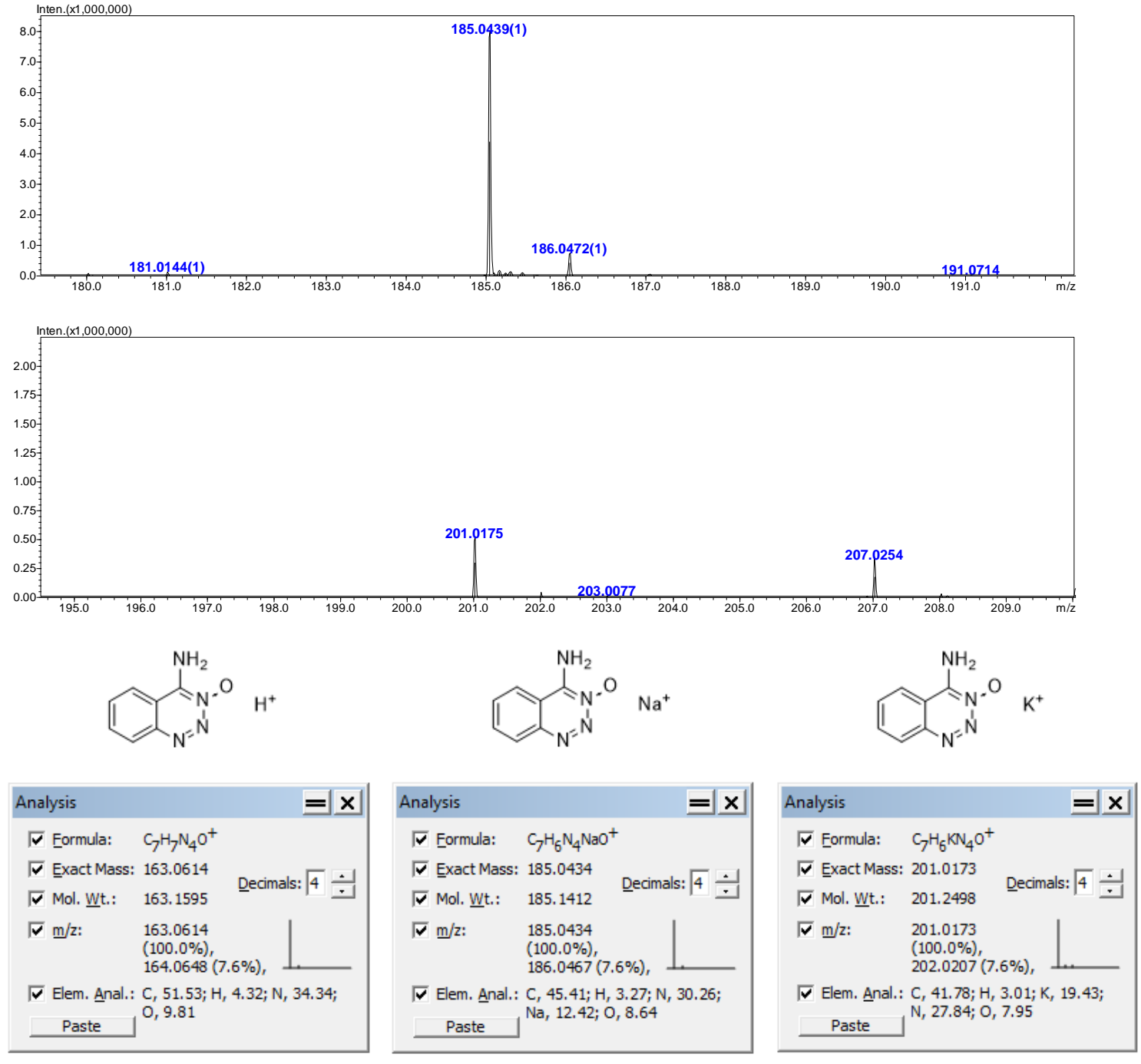

Figure S147. HRMS(ESI+) of 5a.

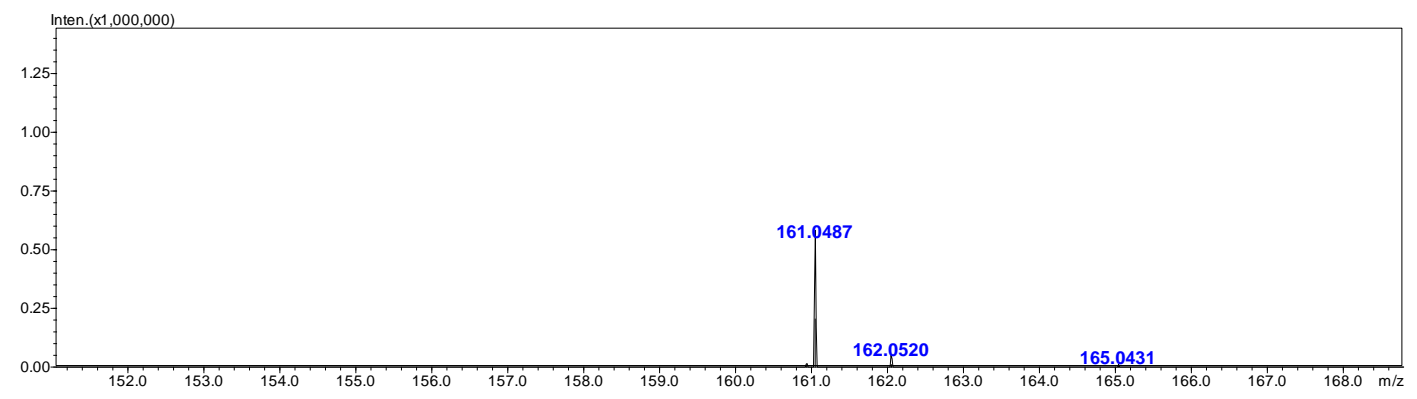




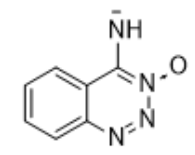

\begin{tabular}{|c|c|}
\hline Analysis & $=x$ \\
\hline$\sqrt{\checkmark}$ Formula: & $\mathrm{C}_{7} \mathrm{H}_{5} \mathrm{~N}_{4} \mathrm{O}^{-}$ \\
\hline$\sqrt{ }$ Exact Mass: & 161.0469 \\
\hline$\sqrt{\checkmark}$ Mol. Wht.: & Decimals: $14 \div$ \\
\hline$\sqrt{ } \mathrm{m} / \mathrm{z}:$ & $\begin{array}{l}161.0469 \\
(100.0 \%), \\
162.0502(7.6 \%),\end{array}$ \\
\hline $\begin{array}{l}\sqrt{\checkmark} \text { Elem. Anal.: } \\
\text { Paste } \\
\end{array}$ & $\begin{array}{l}\text { C, } 52.17 ; \mathrm{H}, 3.13 ; \mathrm{N}, 34.77 \text {; } \\
\text { O, } 9.93\end{array}$ \\
\hline
\end{tabular}

Figure S148. HRMS(ESI-) of 5a.
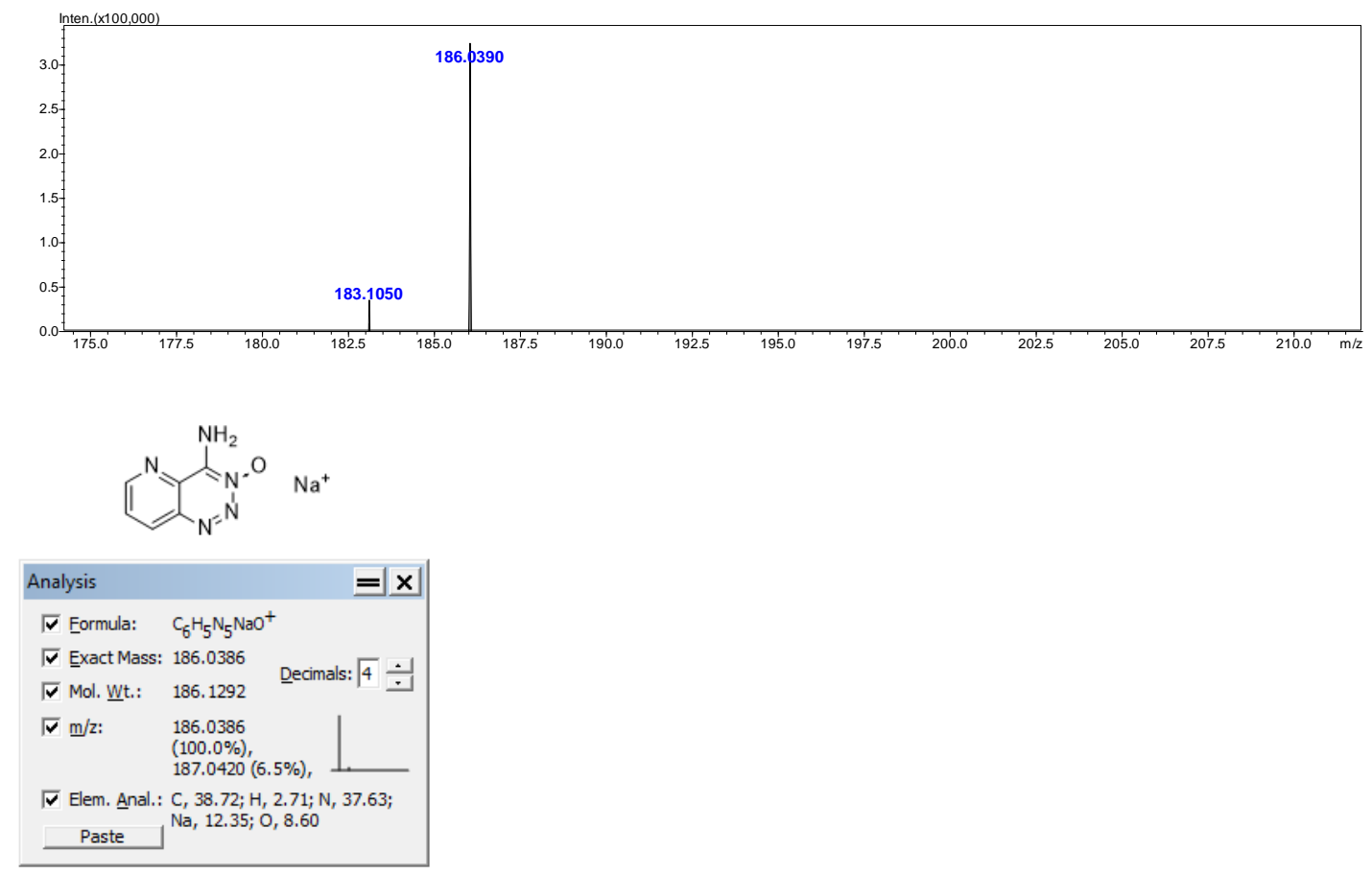

Figure S149. HRMS(ESI+) of 5e. 


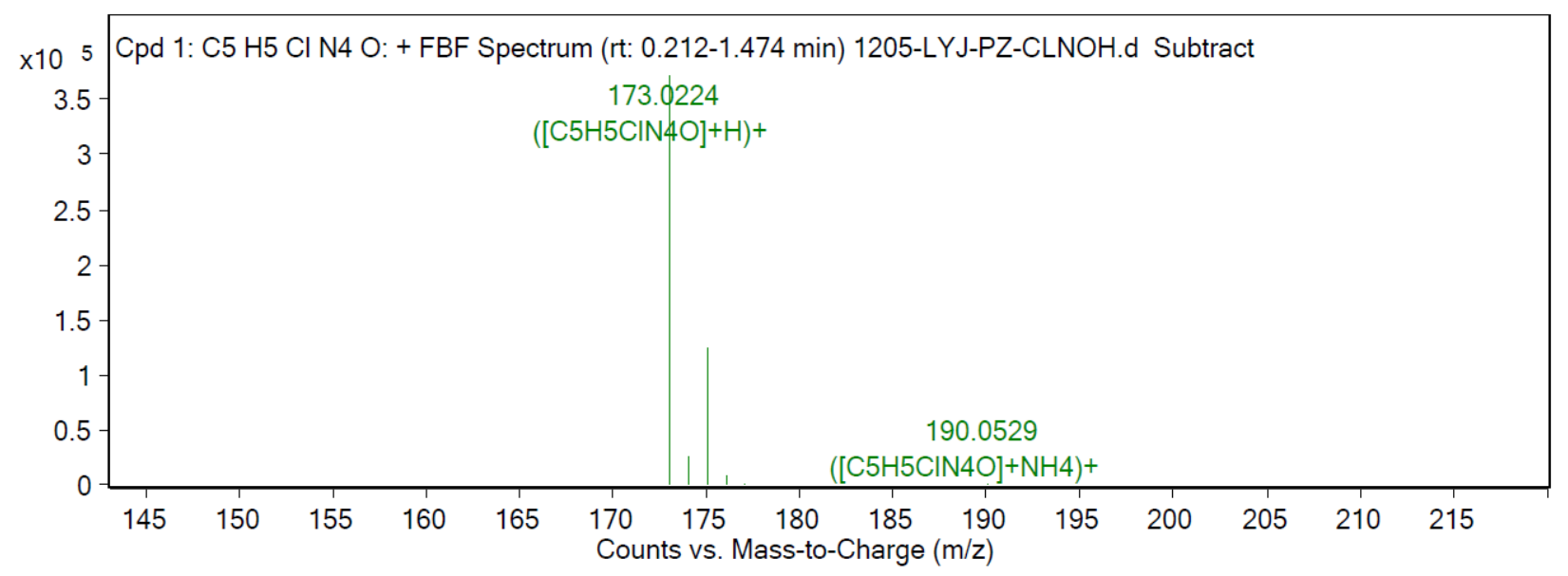

MS Spectrum Peak List

\begin{tabular}{|r|r|r|l|l|}
\hline $\mathrm{m} / \mathrm{z}$ & $\mathrm{Z}$ & Abund & Formula & Ion \\
\hline 173.0224 & 1 & 371863.56 & $\mathrm{C} 5 \mathrm{H} 5 \mathrm{ClN} 4 \mathrm{O}$ & $(\mathrm{M}+\mathrm{H})+$ \\
\hline 174.0253 & 1 & 24692.89 & $\mathrm{C} 5 \mathrm{H} 5 \mathrm{ClN} 4 \mathrm{O}$ & $(\mathrm{M}+\mathrm{H})+$ \\
\hline 175.0196 & 1 & 124230.06 & $\mathrm{C} 5 \mathrm{H} 5 \mathrm{ClN} 4 \mathrm{O}$ & $(\mathrm{M}+\mathrm{H})+$ \\
\hline 176.0223 & 1 & 8490.58 & $\mathrm{C} 5 \mathrm{H} 5 \mathrm{ClN} 4 \mathrm{O}$ & $(\mathrm{M}+\mathrm{H})+$ \\
\hline 177.0229 & 1 & 371.18 & $\mathrm{C} 5 \mathrm{H} 5 \mathrm{ClN} 4 \mathrm{O}$ & $(\mathrm{M}+\mathrm{H})+$ \\
\hline 190.0529 & 1 & 217.92 & $\mathrm{C} 5 \mathrm{H} 5 \mathrm{ClN} 40$ & $(\mathrm{M}+\mathrm{NH} 4)+$ \\
\hline
\end{tabular}

$$
\text { (N) }
$$

\begin{tabular}{|c|c|c|}
\hline Analysis & & $=x$ \\
\hline V Eormula: & $\mathrm{C}_{5} \mathrm{H}_{6} \mathrm{CIN}_{4} \mathrm{O}^{+}$ & \\
\hline$\sqrt{V}$ Exact Mass: & 173.0225 & \\
\hline F Mol. wht: & 173.5795 & als: | \\
\hline$\sqrt{\nabla} \mathrm{m} / z:$ & $\begin{array}{l}\begin{array}{l}173.0225 \\
(100.0 \%) \\
175.0195\end{array}\end{array}$ & |W \\
\hline \multicolumn{3}{|c|}{ 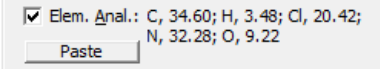 } \\
\hline
\end{tabular}

Figure S150. HRMS(ESI+) of $\mathbf{5 f - 1 .}$ 

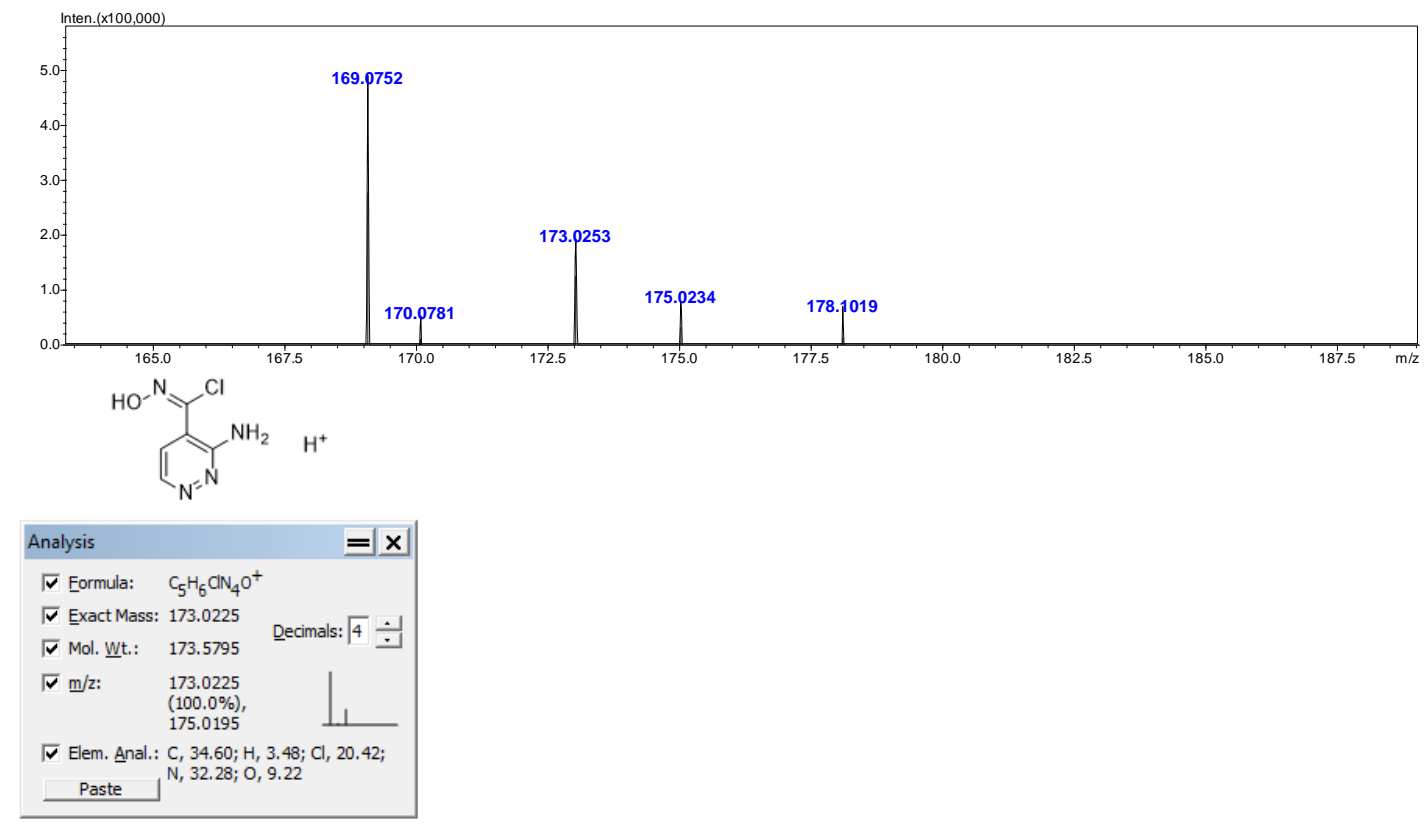

Figure S151. HRMS(ESI+) of 5g-1.
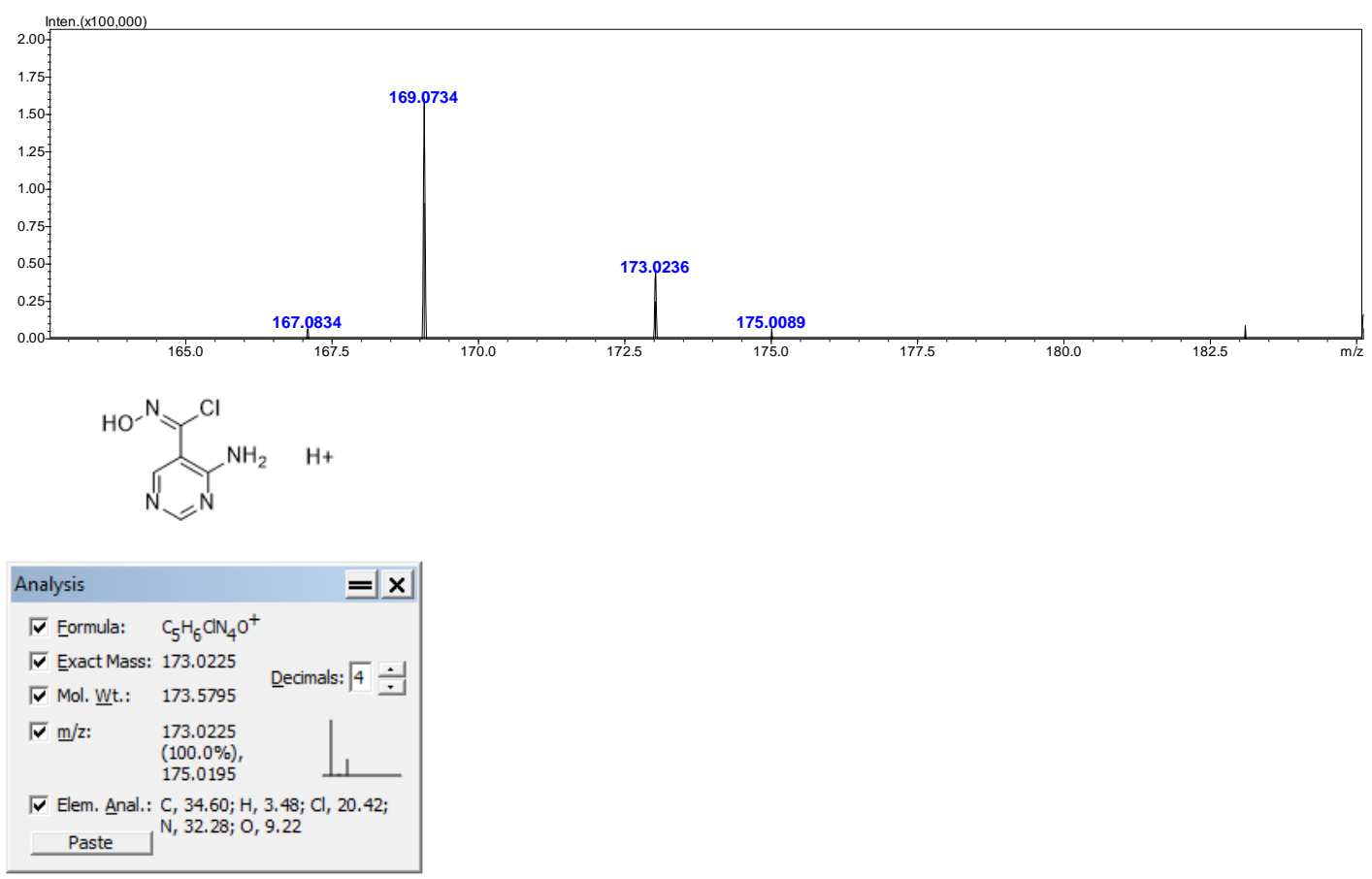

Figure S152. HRMS(ESI+) of 5h-1. 

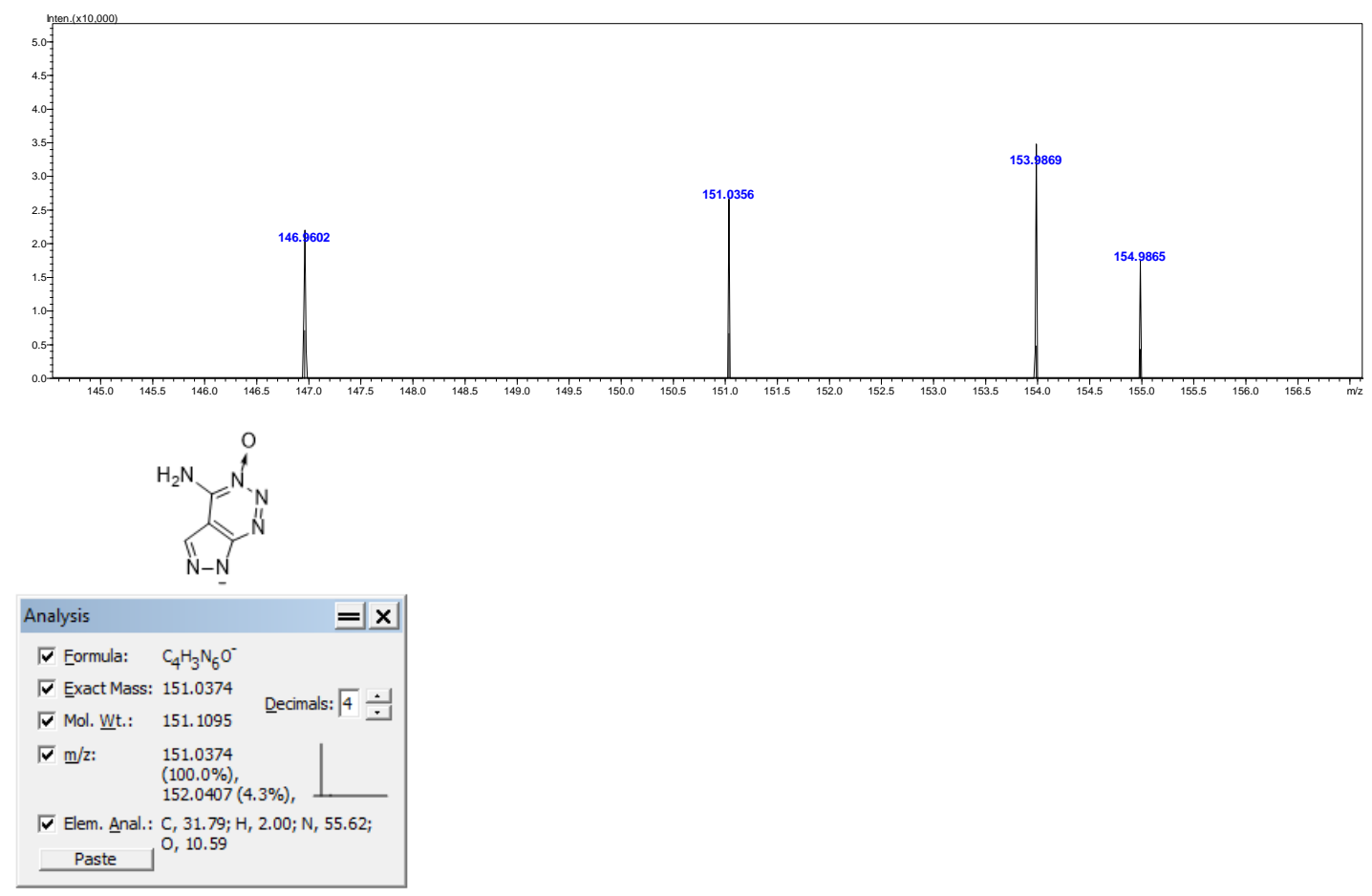

Figure S153. HRMS(ESI-) of $5 \mathbf{i}$.

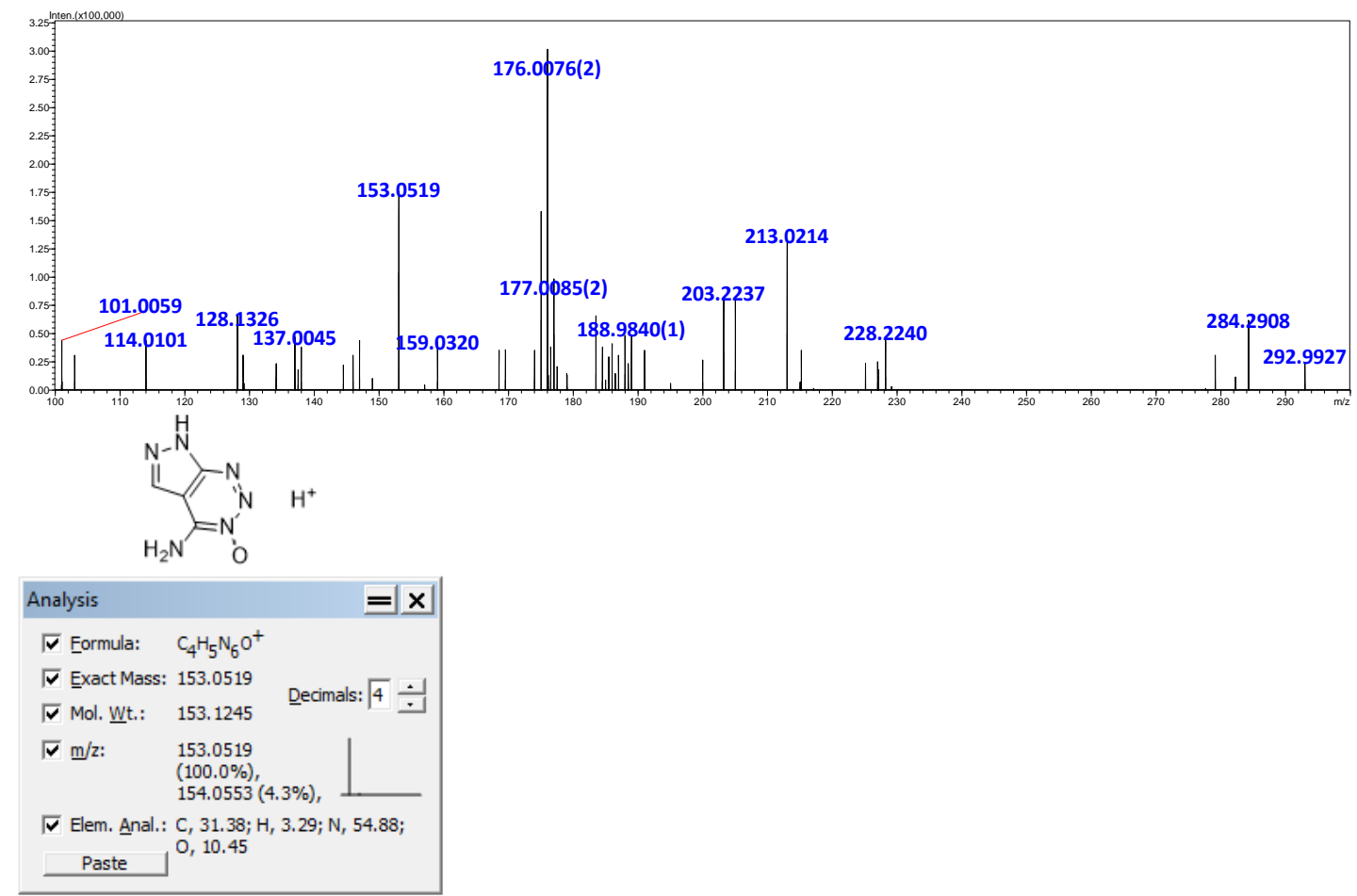

Figure S154. HRMS(ESI+) of 5j. 

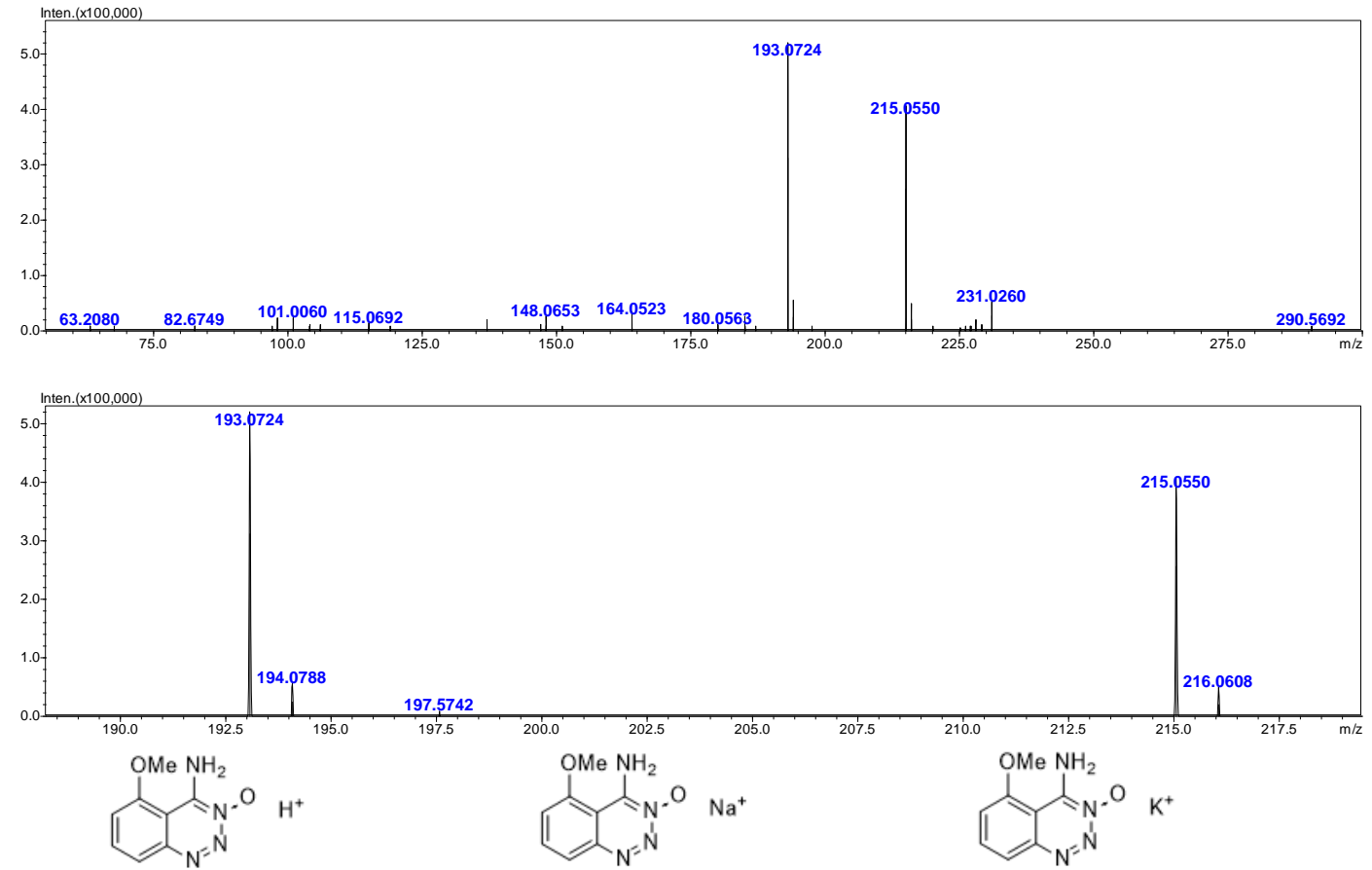

\begin{tabular}{|c|c|}
\hline Analysis & $=x$ \\
\hline$\sqrt{\checkmark}$ Eormula: & $\mathrm{C}_{8} \mathrm{H}_{9} \mathrm{~N}_{4} \mathrm{O}_{2}{ }^{+}$ \\
\hline$\sqrt{\checkmark}$ Exact Mass: & 193.0720 \\
\hline$\sqrt{\nabla}$ Mol. wht: & 193.1855 \\
\hline$\sqrt{\nabla} \mathrm{m} / \mathrm{z}:$ & \begin{tabular}{l|l}
193.0720 & \\
$(100.0 \%)$, & \\
$194.0754(8.7 \%)$ &
\end{tabular} \\
\hline $\begin{array}{l}\sqrt{\checkmark} \text { Elem. Anal.: } \\
\text { Paste } \\
\end{array}$ & $\begin{array}{l}\mathrm{C}, 49.74 ; \mathrm{H}, 4.70 ; \mathrm{N}, 29.00 \\
0,16.56\end{array}$ \\
\hline
\end{tabular}

\begin{tabular}{|c|c|}
\hline Analysis & $=x$ \\
\hline$\sqrt{\checkmark}$ Eormula: & $\mathrm{C}_{8} \mathrm{H}_{8} \mathrm{~N}_{4} \mathrm{NaO}_{2}^{+}$ \\
\hline$\sqrt{\nabla}$ Exact Mass: & 215.0539 \\
\hline$\sqrt{\nabla}$ Mol. $\underline{w}$ t.: & 215.1672 \\
\hline$\sqrt{\nabla} \mathrm{m} / \mathrm{z}:$ & $\begin{array}{l}215.0539 \\
(100.0 \%), \\
216.0573(8.7 \%),\end{array}$ \\
\hline $\begin{array}{l}\sqrt{\checkmark} \text { Elem. Anal.: } \\
\text { Paste }\end{array}$ & $\begin{array}{l}\mathrm{C}, 44.66 ; \mathrm{H}, 3.75 ; \mathrm{N}, 26.04 ; \\
\mathrm{Na}, 10.68 ; 0,14.87\end{array}$ \\
\hline
\end{tabular}

\begin{tabular}{|c|c|}
\hline Analysis & $=x$ \\
\hline$\sqrt{\nabla}$ Eormula: & $\mathrm{C}_{8} \mathrm{H}_{8} \mathrm{KN}_{4} \mathrm{O}_{2}{ }^{+}$ \\
\hline$\sqrt{\nabla}$ Exact Mass: & Decimals: $\sqrt{4} \div$ \\
\hline 『 Mol. wht:: & 231.2758 \\
\hline$\sqrt{\nabla} \mathrm{m} / \mathrm{z}:$ & $\begin{array}{l}231.0279 \\
(100.0 \%), \\
232.0312(8.7 \%), \quad . .\end{array}$ \\
\hline $\begin{array}{l}\text { 『 Elem. Anal.: } \\
\text { Paste } \\
\end{array}$ & $\begin{array}{l}\mathrm{C}, 41.55 ; \mathrm{H}, 3.49 ; \mathrm{K}, 16.91 \\
\mathrm{~N}, 24.23 ; 0,13.84\end{array}$ \\
\hline
\end{tabular}

Figure S155. HRMS(ESI+) of 5k. 


\section{References}

${ }^{1}$ Deng, M.; Feng, Y.; Zhang, W.; Qi, X.; Zhang, Q., A green metal-free fused-ring initiating substance. Nat. Commun. 2019, 10 (1), 1339.

${ }^{2}$ Feng, Y.; Deng, M.; Song, S.; Chen, S.; Zhang, Q.; Shreeve, J. M., Construction of an Unusual TwoDimensional Layered Structure for Fused-Ring Energetic Materials with High Energy and Good Stability. Engineering 2020, 6 (9), 1006-1012.

${ }^{3}$ Tang, Y.; Imler, G. H.; Parrish, D. A.; Shreeve, J. M., Energetic and Fluorescent Azole-Fused 4-Amino-1,2,3triazine-3-N-oxides. ACS Appl. Energy Mater. 2019, 2 (12), 8871-8877.

${ }^{4}$ Gaussian 09, Revision D.01. Frisch, M. J.; Trucks, G. W.; Schlegel, H. B.; Scuseria, G. E.; Robb, M. A.; Cheeseman, J. R.; Scalmani, G.; Barone, V.; Mennucci, B.; Petersson, G. A.; Nakatsuji, H.; Caricato, M.; Li, X.; Hratchian, H. P.; Izmaylov, A. F.; Bloino, J.; Zheng, G.; Sonnenberg, J. L.; Hada, M.; Ehara, M.; Toyota, K.; Fukuda, R.; Hasegawa, J.; Ishida, M.; Nakajima, T.; Honda, Y.; Kitao, O.; Nakai, H.; Vreven, T.; Montgomery, Jr., J. A.; Peralta, J. E.; Ogliaro, F.; Bearpark, M.; Heyd, J. J.; Brothers, E.; Kudin, K. N.; Staroverov, V. N.; Kobayashi, R.; Normand, J.; Raghavachari, K.; Rendell, A.; Burant, J. C.; lyengar, S. S.; Tomasi, J.; Cossi, M.; Rega, N.; Millam, N. J.; Klene, M.; Knox, J. E.; Cross, J. B.; Bakken, V.; Adamo, C.; Jaramillo, J.; Gomperts, R.; Stratmann, R. E.; Yazyev, O.; Austin, A. J.; Cammi, R.; Pomelli, C.; Ochterski, J. W.; Martin, R. L.; Morokuma, K.; Zakrzewski, V. G.; Voth, G. A.; Salvador, P.; Dannenberg, J. J.; Dapprich, S.; Daniels, A. D.; Farkas, Ö.; Foresman, J. B.; Ortiz, J. V.; Cioslowski, J.; Fox, D. J. Gaussian, Inc., Wallingford CT, 2013.

${ }^{5}$ Fukui, K. Acc. Chem. Res. 1981, 14 (12), 363-368.

${ }^{6}$ Fukui, K. J. Phys. Chem. 1970, 74 (23), 4161-4163.

${ }^{7}$ Sheldrick, G. M. Acta Cryst. 2015, A71, 3-8.

${ }^{8}$ Sheldrick, G. M. Acta Cryst. 2015, C71, 3-8.

${ }^{9}$ Dolomanov, O. V.; Bourhis, L. J.; Gildea, R. J.; Howard, J. A. K.; Puschmann, H. J. Appl. Cryst. 2009, 42, 339-341. 